\title{
ICECO-CEL: \\ A COUPLED EULERIAN-LAGRANGIAN CODE FOR ANALYZING PRIMARY SYSTEM RESPONSE IN FAST REACTORS
}

by

C. Y. Wang

BASE TECHNOLOGY

ARGONNE NATIONAL LABORATORY, ARGONNE, ILLINOIS

Prepared for the U. S. DEPARTMENT OF ENERGY under Contract $W-31-109-E n g-38$ 
ICECO-CEL:

A COUPLED EULERIAN-LAGRANGIAN CODE FOR ANALYZING PRIMARY SYSTEM RESPONSE

IN FAST REACTORS

by

c. Y. Wang

Reactor Analysis and Safety Division

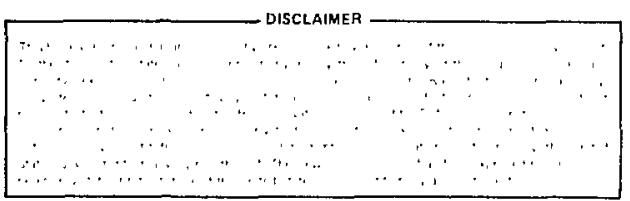

February 1981

$\pi$

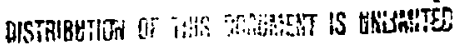


ABSTRACT. ........................................

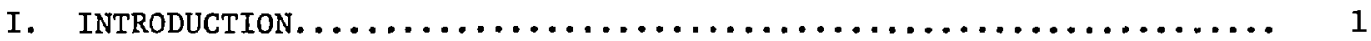

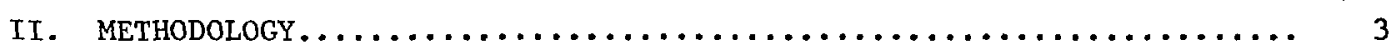

A. Basic Eulerian Hydrodynamics..................... 3

1. The Partial Differential Equations................. 3

2. The Finite-Difference Representations................ 5

3. Particle Movement........................... 6

B. Integral Equations for Perforated Structure.............. 7

C. The Above-Core Hydrodynamics...................... 8

1. Fluid Motion Inside Coolant Passageways................ 9

2. Fluid Motion Above the Perforated Structure............. 11

3. Fluid Motion Below the Perforated Structure............. 13

D. Moving Cover and Sodium Spillage.................... 16

E. Flow Near Primary Vessel and Deformable Internals........... 20

1. The Fluid-Vessel Interface....................... 20

2. Interaction of Fluids with Deformable Internals.......... 21

F. Treatment of the Bubble-Coolant Interface and Free Surface.... 22

1. Marker Particle and Volume Fraction Methods............ 22

2. Free-Surface Boundary Conditions.................. 23

G. Wave Transient in Cavitated Liquids.................. 25

H. Heat Transfer and Fuel-Coolant Interaction............... 26

I. The Equation of State of the Media................... 27

J. Differencing Schemes and Convective Fluxes............... 29

K. Lagrangian Formulation for Continua and Structures.......... 31

1. Quadrilateral Continuum...................... 32

a. Nodal Force............................ 32

b. Stress and Yield Condition.................. 35

2. Shell Element........................... 36

L. Stability Consideration....................... 38

M. Solution Procedure.............................. 40 


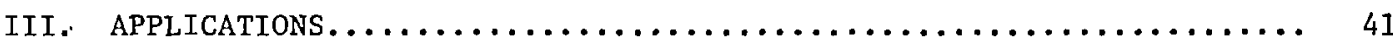

A. Response of Pool-Type LMFBR...................... 41

B. Above-Core Hydrodynamics Induced by Upper Internals......... 42

1. The Effect of Upper Internal Structure on the Wave Prop-

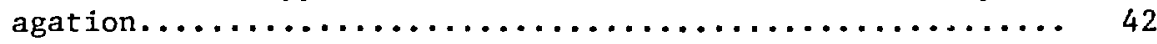

2. Optional Mode1.ings for the Upper Internal Structures...... 43

C. Sodium Spillage From the Primary Containment............ 43

1. Sodium Spillage During an Energetic HCDA............ 43

2. Integrated Analysis of Containment Response and Sodium Spillage................................... 44

D. The Effect of Core-Support-Structure Openings on the Containment Response............................... 45

E. Heat Transfer - The Effect of Condensation on the Pressure Loading................................... 46

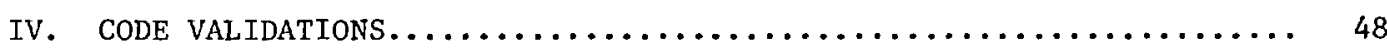

A. Shock-Tube Problem........................... 48

B. ANL Experiment on Bubble Expansion and Contraction.......... 49

C. SRI Test on Wave Propagation in an Elastic-Plastic Pipe...... 50

D. SNR-300 Tests on Wave Propagations in Primary Containment..... 51

1. The Thick-Vessel Model........................ 51

2. The Thin-Vessel Configuration.................. 54

3. The Double-Vesse1 Mode1....................... 56

E. UKAEA-JRC COVA Experiment of High-Energy Excursion Involving Shock Waves and Cavitations....................... 57

1. Rigid-Wall Solution........................ 59

2. Deformable-Wall Solution....................... 60

F. SRI Complex Vesse1 Test SM-2 on Structural Response to á

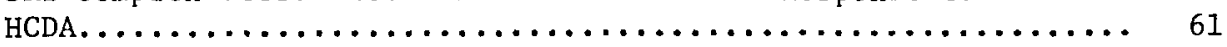

V. THE COMPUTER PROGRAM. ........................... 63

A. Cell Identification............................ 63

1. General Cell Flag........................... 63

2. Boundary-Cell Flag............................ 64

3. Surface-Cell Flag............................... 65

a. Conventional Flagging Scheme................... 65

b. Detailed Flagging scheme...................... 66 
B. Other Information............................. 66

1. Surface-Cell Velocity Calculation................. 66

2. Marker-Particle Setup and Slug Impact............... 67

3. The Computing Domain and Modeling Technique............ 68

4. Hydrodynamic-Structure Connectivity................ 69

C. Computer Output............................ 71

1. Input verification Printout.................... 71

a. Data for Hydrodynamics..................... 71

b. Data for Structural Dynamics................. 71

2. General Printout........................... 72

a. A11 Problems............................. 72

b. Moving-Cover and Sodium-Spillage Analysis......... 72

3. Optional printout......................... 73

a. Detajled Cell-Variable Output................ 73

b. Particle-Position Output.................... 7.3

c. Boundary $-F$ lag Output....................... 73

d. Deformabile-Internal Output................. $7 \dot{4}$

4. Pictorial display............................ 74

a. FILM Output.............................. 74

b. Results for Calcomp plot.................... 74

D. Program Execution and Limitations.................. 75

1. Changes of Common Blocks for Saving Computer Storage...... 75

2. Adjustment of Subroutines Needed for Saving the Computer

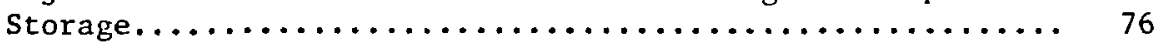

3. Limitations.............................. 76

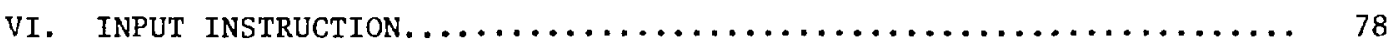

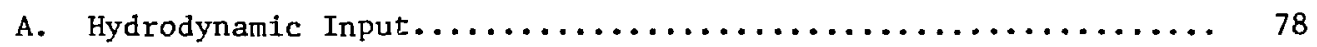

B. Finite-Element Structural Dynamics Input................. 124

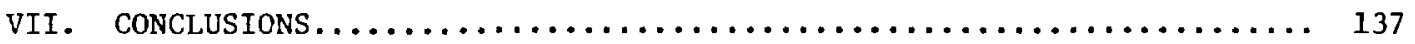

ACKNOWLEDGMENTS. ................................. 138

REFERENCES........................................ 139 


\section{LIST OF FIGURES}

\begin{tabular}{|c|c|c|}
\hline Number & Title & $\underline{\text { Page }}$ \\
\hline 1. & 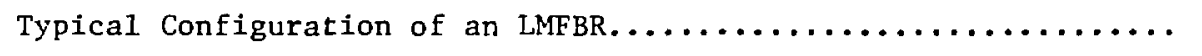 & 142 \\
\hline 2. & 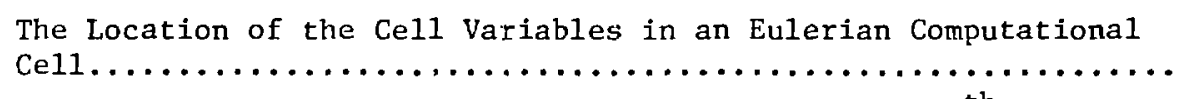 & 142 \\
\hline 3. & $\begin{array}{l}\text { Linear Interpolation for } \mathrm{U}_{\mathrm{K}} \text { and } \mathrm{v}_{\mathrm{K}} \text { with Position of } \mathrm{K}^{\text {th }} \text { Particle } \\
\text { as Shown } \ldots \ldots \ldots \ldots \ldots \ldots \ldots \ldots \ldots \ldots \ldots \ldots \ldots \ldots \ldots \ldots \ldots \ldots \ldots \ldots \ldots \ldots \ldots \ldots\end{array}$ & 143 \\
\hline 4. & Eulerian Computing Mesh for Upper Internal Structure........... & 143 \\
\hline 5. & $\begin{array}{l}\text { Control Volume for Derivation of Axial Momentum Equation Inside } \\
\text { Coolant Passageways } . \ldots \ldots \ldots \ldots \ldots \ldots \ldots \ldots \ldots \ldots \ldots \ldots \ldots \ldots \ldots \ldots \ldots \ldots \ldots \ldots\end{array}$ & 143 \\
\hline 6. & 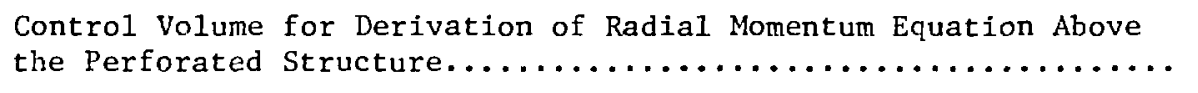 & 144 \\
\hline 7. & 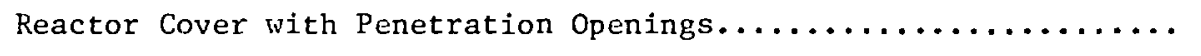 & 144 \\
\hline 8. & $\begin{array}{l}\text { Control Volume for Radial Momentum Equation Underneath the } \\
\text { Cover } \ldots \ldots \ldots \ldots \ldots \ldots \ldots \ldots \ldots \ldots \ldots \ldots \ldots \ldots \ldots \ldots \ldots \ldots \ldots \ldots \ldots \ldots \ldots \\
\end{array}$ & 144 \\
\hline 9. & $\begin{array}{l}\text { Control Volume for Axial Momentum Equation Underneath the } \\
\text { Cover } \ldots \ldots \ldots \ldots \ldots \ldots \ldots \ldots \ldots \ldots \ldots \ldots \ldots \ldots \ldots \ldots \ldots \ldots \ldots \ldots \ldots \ldots \ldots \ldots \ldots \ldots \ldots\end{array}$ & 144 \\
\hline 10. & 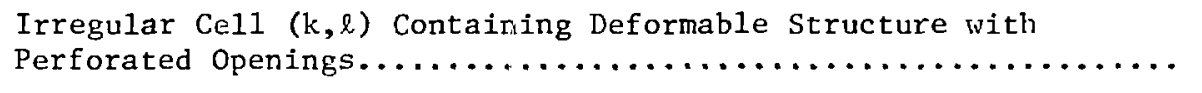 & 144 \\
\hline 11. & Velocity Calculation in Vicinity of a Surface Cell............ & 145 \\
\hline 12. & Conventional Equation of State for Cavitated Fluid............. & 145 \\
\hline 13. & Zone Configuration for Finite-Difference Equations............ & 145 \\
\hline 14. & Numbering Scheme for Calculating Node Forces.............. & 145 \\
\hline 15. & 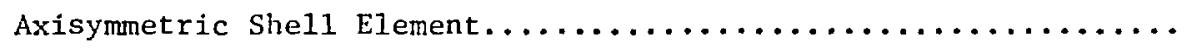 & 146 \\
\hline 16. & Flow Chart Representing the Computational Procedure............ & 146 \\
\hline 17. & Initial Configuration of the Pool-Type LMFBR................ & 147 \\
\hline 18. & Reactor Configurations at. Several Different Times............. & 147 \\
\hline 19. & Radial Displacement History of the Upper End of the Core Barrel.. & 148 \\
\hline 20. & Initial Reactcr Configuration with the Upper Internal Structure.. & 148 \\
\hline 21. & Comparison of Total Force on the Reactor Cover.............. & 149 \\
\hline 22. & $\begin{array}{l}\text { Reactor Configurations at Three Different Times (Upper Internal } \\
\text { Modeled as Perforated Structure) } \ldots \ldots \ldots \ldots \ldots \ldots \ldots \ldots \ldots \ldots \ldots \ldots \ldots \ldots\end{array}$ & 149 \\
\hline 23. & $\begin{array}{l}\text { Reactor Configurations at Three Different Times (Upper Internal } \\
\text { Modeled by Rigid Obstacles) } \ldots \ldots \ldots \ldots \ldots \ldots \ldots \ldots \ldots \ldots \ldots \ldots \ldots \ldots \ldots \ldots\end{array}$ & 150 \\
\hline 24. & $\begin{array}{l}\text { Reactor Configurations at Three Different Times (UIS Passageways } \\
\text { were Plugged) } \ldots \ldots \ldots \ldots \ldots \ldots \ldots \ldots \ldots \ldots \ldots \ldots \ldots \ldots \ldots \ldots \ldots \ldots \ldots\end{array}$ & 150 \\
\hline 25. & $\begin{array}{l}\text { (a) Configuration of a Simplified Reactor (Top Penetration } \\
\text { Initially Closed), (b) Configuration of } 50 \text { ms Showing Coolant } \\
\text { Spillage from Top Penetration and from Vesse1-Head Junction...... }\end{array}$ & \\
\hline
\end{tabular}




\section{LIST OF FIGURES (contd.)}

Number

26. Sodium Velocities at Top Penetration and Side Opening......... 151

27. Total Spillage from Top Penetration and Side opening.......... 151

28. Initial Configuration Used for Containment and Sodium-Spillage Analysis...................................... 152

29. Reactor Configurations at Two Different Times.............. 152

30. Initial Configuration Used in the Analysis............... 153

31. Configurations at various Times....................... 153

32. Pressure Loadings in the Reactor Lower Plenum.............. 153

33. Axial Deformation of Bottom Vessel................... 154

34. Model of a Single Subassembly and Pressure Histories.......... 154

35. Marker-Particle Configurations (with Condensation)........... 155

36. Schematic of Shock Tube (not to Scale)................... 155

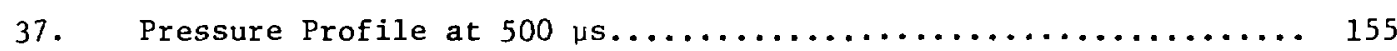

38. Pressure Profiles at $500 \mu$ for Various Time Steps............ 156

39. Simplified Test Apparatus for Bubble Motion............... 156

40. Bubble and Coolant Configurations at Seven Different Times...... 157

41. Experimental Bubble Configurations at Seven Different Times..... 157

42. Pressure Histories at Location A.................. 157

43. Configuration of the Elastic-Plastic Pipe................ 158

44. Pressure Histories at Various Locations in the Elastic-Plastic

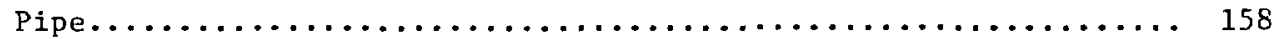

45. Radial Deformations at Six Positions ( - ICECO; -- SRI Test)... 158

46. Test Configurations of the SNR-300 Reactor Models............ 159

47. Comparison of Pressure at $r=10 \mathrm{~cm}$ and $z=0$. (Delay of

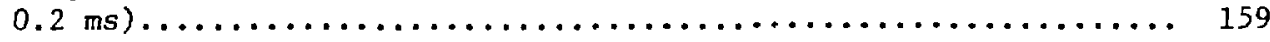

43. Comparison of Pressure at $r=25.5 \mathrm{~cm}$ and $z=36 \mathrm{~cm}$. (Delay of

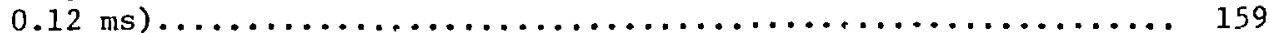

49. Reactor Configurations at Three Different Times (No Spillage).... 159

50. Reactor Configurations at Three Different Times (with Spillage).. 160

51. Comparison of the Permanent Vessel Deformations (Thin-Vessel Mode1)....................................... 160

52. Comparison of the Permanent Vessel Deformation (Double-Vessel

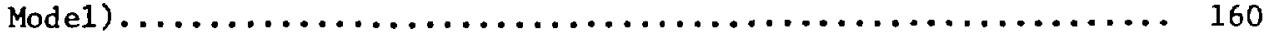

53. (a) Test Model of the CoVA Overstrong Vessel Experiment: (b-d) Impulses on the Floor, Wall, and the Roof, (e) Configuration at

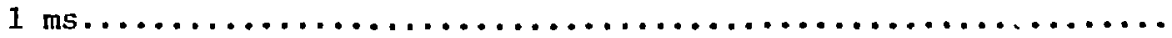




\section{LIST OF FIGURES (contd.)}

Number

$\underline{T i t 1 e}$

Page

54. Comparison of Pressure Histories at Roof Gauge \#18 ( $r=160 \mathrm{~mm}) \ldots 162$

55. Pressure and Impulse Histories for Floor Gauge $\# 1(r=24 \mathrm{~mm}) \ldots 162$

56. Impulses at Floor Gauges........................ 162

57. SM-2 Test Model with Instrumentation................. 162

58. Configurations at Two Different Times.................. 163

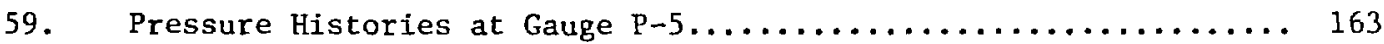

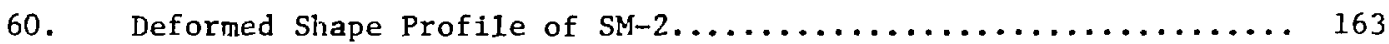

61. Different Type of Flags at the Deformable Boundaries......... 164

62. Conventional Cell Flags Used Near the Free Surface........... 16:

63. Detailed Cell Flags Used Near the Free Surface.............. 165

64. Marker Particle Setup............................... 165

65. Computing Mesh for Problems with Flexible External Boundaries.... 165

66. Computing Mesh for Problems with Deformable Internals......... 166

67. Numbering System for Treating Fluid-Structure Interaction with Multiple Load Lines............................. 166

68. Component Number for the Time-History Plot $\ldots \ldots \ldots \ldots \ldots \ldots \ldots \ldots . \ldots$ 


\section{LIST OF TABLES}

1. Source Characteristics for the Thick-Vesse: Model........... 52

2. Source Characteristics for the Thin-Vessel Model............. 53 
ICECO-CEL

\section{A COUPLED EULERIAN-LAGRANGIAN CODE \\ FOR ANALYZING PRIMARY SYSTEM RESPONSE \\ IN FAST REACTORS}

by

C. Y. Wang

\section{ABSTRACT}

This report describes a coupled Eulerian-Lagrangian code, ICECO-CEL, for analyzing the response of the primary system during hypothetical core disruptive accidents. In the analysis, the implicit Eulerian method is used to calculate the fluid motion so that large fluid distortion, two-dimensional sliding interface, flow around corners, flow through coolant passageways, and out-flow boundary conditions can be adequately treated. The explicit Lagrangian formulation is employed to compute the response of the containment vessel and other elastic-plastic solids inside the reactor containment. Large displacements, as well as geometrical and material nonlinearities are considered in the analysis. Marker particles are utilized to define the free surface or the material interface, and to aid the visualization of the fluid motion.

In this report the basic equations and numerical techniques used in the Eulerian hydrodynamics and Lagrangian structural dynamics are described in detail. Treatment of the above-core hydrodynamics, sodium spillage, fluid cavitation, freesurface boundary conditions and heat transfer are also presented, together with the assumptions and limitations of the mathematical model used in the analysis. Many examples are given to illustrate the capabilities of the computer code. Comparisons of the code predictions with available experimental data are also made. 


\section{INTRODUCTION}

Although the occurrence of energy excursions in a Liquid Metal Fast Breeder Reactor (LMFBR) has extremely low probability, safety analysis of LMFBR requires an analysis on the capability of the primary containment system to sustain the severe consequences of Hypothetical Core Disruptive Accidents (HCDAs). In the USA the safety criteria [1] requires that the primary containment be remained intact throughout the event of the energy excursion, and the fissiongas products and other harmful radioactive materials be prevented from release to the surrounding environs.

To accomplish this objective safety research groups throughout the world have been developing computer codes for analyzing the transient response of LMFBR subjected to postulated accident loads. Many of the codes currently in use are based on the Lagrängian formulations for both fluid and structural analysis, employing explicit time integration algorithm. The first Lagrangian Containment Code, REXCO-H [2], was developed in 1969 at Argonne National Laboratory. Since then a number of Lagrangian containment codes [3-6] have evolved. Other computer codes developed for different purposes [7-10] have also been applied to safety problems related to IMFBR containment.

Despite the fact that Lagrangian computer codes have been successfully used for analyzing the primary containment response under HCDAs. Their analyses are still limited to the early stages of the excursion, because excessive grid distortions will often deteriorate the accuracy of the numerical results. With the increasing concern for safety design of various LMFBRs and wide range of possible energy excursions, the issues of extended fluid motions accompanying by complex physical phenomena have gathered increasing importance. For instance, in a loop-type LMFBR shown in Fig. 1 concerns arose recently over the above-core hydrodynamics induced by the upper internal structure, the possibility of sodium spillage and its resulting sodtum fires in the secondary containment, the expansion and contraction of the core-gas bubble, and the role of fluid cavitation on the containment response.

To assist the Lagrangian codes for analyzing the primary-system response during an HCDA a computer code, ICECO-CEL, is developed, where ICECC and CEL denote 
Implicit Continuous-Fluid Eulertan Containment Code and Coupled Eulerian ingrangian, respectively. This code is an extension of the ICECO code developed [11-14] early. It uses the implicit Eulerian hydrodynamic technique to treat the fluid motion, while employs explicit Lagrangian method to analyze the structural dynamics. These two analyses are coupled together at the interface where boundary conditions are rigorously satisfied. Thus, this program combines the advantrges of both Eulerian and Lagrangiari coordinate systems. The Eulerian coordinate system remains fixed in space, and can calculate violent fluid motion and fluid flow around irregular objects. The Lagrangian coordinate system moves with the material and can record the histories of the elasticplastic solid for use in the constitutive equations of complex reactor structures.

In this report the governing equations for treating both fluids and solids are described in detail. Many sample problems are given and their results are discussed. Input instruction are also included. 


\section{METHODOLOGY}

\section{A. Basic Eulerian Hydrodynamics}

\section{The Partial Differential Equations}

The basic differential equations used in the code are the conservation equations of continuum mechanics. Only nonturbulent flow is considered, and no external energy source is assumed to exist inside the flow region. Thus, the mass, momentum, and energy equations are:

$$
\begin{aligned}
& \frac{\partial \rho}{\partial t}+\frac{\partial}{\partial \mathbf{x}_{i}}\left(\rho u_{i}\right)=0, \\
& \frac{\partial}{\partial t}\left(\rho w_{i}\right)+\frac{\partial}{\partial x_{j}}\left(\rho w_{i} w_{j}\right)=\frac{\partial}{\partial x_{i}} \sigma_{i j}+\rho g_{i}, \\
& \frac{\partial}{\partial t}(\rho E)+\frac{\partial}{\partial x_{i}}\left(\rho w_{i} E\right)=\frac{\partial}{\partial x_{i}}\left(\sigma_{i j} w_{j}\right)+\frac{\partial}{\partial x_{i}}\left(\mu B \frac{\partial I}{\partial x_{i}}\right)+\rho g_{i} w_{i},
\end{aligned}
$$

in which

$$
\Sigma=I+\frac{1}{2} w_{i} w_{i}
$$

In Eqs. $(1-4) \rho$ is the density; the time; $w_{i}$ the velocity component; $g_{i}$ the component of gravity acceleration; $\mu$ the viscosity coefficient; $E$ the specific total energy; I is the specific internal energy. The temperature in the heat conduction term has been eliminated by means of the constant coefficient B; $\sigma_{i j}$ is the stress tensor which is a function of the scalar pressure $p$ and a deviatoric stress tensor $\bar{\sigma}_{i j}$, i.e.

$$
\sigma_{i j}=-p \delta_{i j}+\bar{\sigma}_{i j}
$$

where $\delta_{i j}$ is Kronecker delta.

The deviatoric stress tensor can be written as

$$
\bar{\sigma}_{i j}=\mu e_{i j}+\frac{1}{2} \lambda e_{\ell \ell} \delta_{i j},
$$

and 


$$
e_{i j}=\frac{\partial w_{j}}{\partial x_{i}}+\frac{\partial w_{1}}{\partial x_{j}},
$$

in which $\lambda$ is another viscosity coefficient. Note that tensor notation is employed, so that repeated indices denote summations.

The coolant motion inside the reactor containment is assumed to be axisymmetric. Substitution of Eqs. (5) and (6) into (2) and (3). The mass, momentum, and energy equations are:

$$
\begin{aligned}
& \frac{\partial \rho}{\partial t}+\frac{1}{r} \frac{\partial \rho u r}{\partial r}+\frac{\partial \rho v}{\partial z}=0, \\
& \frac{\partial \rho u}{\partial t}+\frac{1}{r} \frac{\partial \rho u^{2} r}{\partial r}+\frac{\partial \rho u v}{\partial z}=-\frac{\partial}{\partial r}(p+q)+\mu \frac{\partial}{\partial z}\left(\frac{\partial u}{\partial z}-\frac{\partial v}{\partial r}\right), \\
& \frac{\partial \rho v}{\partial t}+\frac{1}{r} \frac{\partial \rho u v r}{\partial r}+\frac{\partial \rho v^{2}}{\partial z}=\rho g-\frac{\partial}{\partial z}(p+q)-\frac{\mu}{r} \frac{\partial}{\partial r}\left[r\left(\frac{\partial u}{\partial z}-\frac{\partial v}{\partial r}\right)\right], \\
& \frac{\partial \rho E}{\partial t}+\frac{1}{r} \frac{\partial \rho u E r}{\partial r}+\frac{\partial \rho v E}{\partial z}=\rho v g+\frac{1}{r} \frac{\partial}{\partial r}\left\{r \left[B \mu \frac{\partial I}{\partial r}-p u-\left(\frac{\lambda}{\lambda+2 \mu}\right) q u\right.\right. \\
& + \\
& \left.\left.+\frac{\mu}{2} \frac{\partial}{\partial r}\left(2 u^{2}+v^{2}\right)+\mu v \frac{\partial u}{\partial z}\right]\right\}+\frac{\partial}{\partial z}\left\{B \mu \frac{\partial I}{\partial z}-p v-\left(\frac{\lambda}{\lambda+2}\right) q v\right. \\
& \left.+\frac{\mu}{2} \frac{\partial}{\partial z}\left(u^{2}+2 v^{2}\right)+\mu u \frac{\partial v}{\partial r}\right\},
\end{aligned}
$$

where

$$
q \equiv-(\lambda+2 \mu)\left(\frac{1}{r} \frac{\partial u r}{\partial r}+\frac{\partial v}{\partial z}\right),
$$

in which $u$ and $v$ are the velocity cumponents in the $r$ and $z$ directions, respectively.

The equation of state is of the form

$$
\mathrm{p}=\mathrm{f}(\rho, I) \quad
$$

The differential equations mentioned above are written in cylindrical coordinates and are used in the implicit Eulerian hydrodynamic methods [11,15]. Here, we refer to these equations as well as the governing finite-difference equation for the purpose of completeness. 


\section{The Finite-Difference Representations}

Like most Eulerian hydrodynamic methods $[11,15,17]$, the equations of mass and monentum are expressed in the advanced-time forms using an implicitdifference representation. Figure 2 shows an Eulerian finite-difference mesh, illustrating the centering of the physical variables relative to a typical cell. The velocities are defined at the cell boundaries with $u$ at $i \pm \frac{1}{2}$ and $v$ at $j \pm \frac{1}{2}$. Other field variables are all defined at the cell center.

To simplify the analysis, the description of the basic hydrodynamics and the detailed derivation of finite-difference expressions of mass and momentum equations will not be presented here, but are given in Refs. [11,13,15].

The equation of state of the coolant is

$$
\bar{p}_{i, j}=p_{i, j}^{n}+c_{i, j}^{n}\left(\rho_{i, j}^{n+1}-\rho_{i, j}^{n}\right) \text {, }
$$

in which

$$
c_{i, j}^{n}=\left(\frac{\partial p}{\partial \rho}\right)_{i, j} \quad,
$$

is closely related to speed of sound; $\bar{p}_{i, j}$ is the advanced-time pressure; the superscripts $n+1$ and $n$ will denote the $(n+1)-t h$ and $n-t h$ time cycles in the following development; variables lacking superscripts will represent values of the $n-t h$ cycle.

Thus, eliminating the mass fluxes between the mass and momentum equations, in conjunction with the equation of state relating the advanced-time pressures and densities, results in a Poisson's equation governing the advanced-time presisures:

$$
\begin{aligned}
\bar{p}_{i, j}\left[\frac{1}{c_{i, j}^{n}}\right. & \left.+2 \theta \psi \delta t^{2}\left(\frac{1}{\dot{o r} r^{2}}+\frac{1}{\delta z^{2}}\right)\right]=G_{i, j}+\theta \psi \delta t^{2}\left[\frac{r_{i-\frac{1}{2}} \bar{p}_{i-1, j}+r_{i+\frac{i}{2}} \bar{p}_{i+1, j}}{r_{i} \delta r^{2}}\right. \\
& \left.+\frac{\bar{p}_{i, j-1}+\bar{p}_{i, j+1}}{\delta z^{2}}\right]
\end{aligned}
$$

where 


$$
G_{i, j}=G\left(R_{I \pm \frac{1}{2}, j} ; s_{i, j \pm \frac{1}{2}} ; u, v, p, \rho, c^{-I}, \ldots . .\right) \text {, }
$$

is the source term of the Poisson's equation which is evaluated from previous cycle values; $\mathrm{R}$ and $\mathrm{S}$ are the source terms of the momentum equations in the and $z$ directions, respectively; $\delta t$ is the time step, $\delta \mathrm{r}$ and $\delta z$ are respectively, the dimensions of a cell in the radial and axial directions; $\bar{p}$ is the advancedtime pressure, corresponding to the pressure at the $(n+1)$ th cycle with superscript $n+1$ neglected, $\psi$ and $\theta$ are the weighting constants of the momentum and mass equations which are utilized for the numerical treatment, as suggested in the ICE technique. These constants, with magnitudes between 0.0 and 1.0 , represent the relative level of time centering of the pressure-gradient and massflux terms. For $\psi=1.0$ the technique is purely implicit [15].

Noted that Eq. (14) is the governing differencs equation for the advanced-time pressure in a cell completely filled with the fluid. It consists of five unknowns and is solved by the iteration technique.

If the fluid is bounded by the rigid walls and the wall boundaries coincide with the Eulerian grid lines, Eq. (14) is sufficient to determine the advanced-time pressure field. However, if the computational region involving flexible vessel or deformable internals, modification of source-term calculations is needed. For more details, the reader should refer to Ref. [13].

\section{Particle Movement}

After solving for the advanced-velocity field and other field variables, a new configuration of the flow field is generated by moving the marker particles to their new positions:

$$
r_{k}^{n+1}=r_{k}^{n}+u_{k} \delta t ; z_{k}^{n+1}=z_{k}^{n}+v_{k} \delta t,
$$

where $u_{k}$ and $v_{k}$ denote the velocity components for the kth particle. The values of $u_{k}$ and $v_{k}$ are obtained by averaging from the $u$ and $v$ fields, respectively. For example, if particle $\mathrm{k}$ is located within the cel $\mathrm{l}$ where velocity components are defined at the nearby locations (see Fig. 3), $u_{k}$ and $v_{k}$ can be calculated by the area-weighting method: 


$$
u_{k}=\frac{A_{1} u_{1}+A_{2} u_{2}+A_{3} u_{3}+A_{4} u_{4}}{\delta r \delta z},
$$

and

$$
v_{k}=\frac{B_{1} v_{1}+B_{2} v_{2}+B_{3} v_{3}+B_{4} v_{4}}{\delta r \delta z} .
$$

\section{B. Integral Equations for Perforated Structure}

Nuclear reactors usually have structure components with small coolant passageways, such as the core-support structure, upper internal structure and the reactor cover. The equations governing the fluid motion near these structures can be formulated rigorously if they have only one-dimensional movements. In general, the coolant flow through the coolant passageways can be analyzed by the conventional Eulerian and Arbitrary Lagrangian-Eulerian methods using various narrow cells to approach the size of the passageways. However, the resulting analysis has three drawbacks: (1) a large number of computational cells is required; (2) the time steps, usually proportional to the width of the narrow cell in accordance with the modified Courant stability condition, are limited to very small values; (3) undesirable errors may be induced if the variation of the cell widtl becomes too drastic.

To eliminate the difficulties mentioned previously, a hydradynamic method for treating perforated structure is developed. It allows the use of large (wide) cells over the entire computational region. Thus, numerical calcuiations can be performed with fewer cells and thereby larger time steps. Consequently, the computational efficiency is optimized.

For the developments of flow through perfurated structure it is desirable to replace the differential equations by the analogous integral equations, obtained by integrating over the respective control volume, $v$, and then converting the volume integral of divergences to surface integrals over the control surfaces. Thus, in integral form, the mass, momentum, and energy equations for a non-heatconducting fluid are [16]: 


$$
\begin{aligned}
& \frac{\partial}{\partial t} \int_{V} \rho d V+\int_{S} \rho w_{i} n_{i} d S=0, \\
& \frac{\partial}{\partial t} \int_{V} \rho w_{i} d V+\int_{S} \rho w_{i} w_{j} n_{j} d S=\int_{S} \sigma_{i j} n_{j} d S+\int_{V} \rho g_{i} d V,
\end{aligned}
$$

and

$$
\frac{\partial}{\partial t} \int_{V} \rho E d V+\int_{S} \rho w_{i} E n_{i} d S=\int_{S} \sigma_{i j} w_{j} n_{i} d S+\int_{V} \rho g_{i} w_{i} d V,
$$

where $n_{i}$ is the outward normal of the surface $s_{i}$.

The control volumes employed for obtaining the radial velocity $u$, axial velocity $v$, and specific density $\rho$ are essentially the same as those of the ICE technique. In other words, the control volume for derivation of $u$ is centered at $\left(i+\frac{1}{2}, j\right)$, while control volumes for derivations of $v$ and $\rho$ are centered, respectively, at $\left(i, j+\frac{1}{2}\right)$, and $(i, j)$. Also, to simplify the analysis of flow through the perforated structure we assume that the flow inside the coolant passageways is one-dimensional. Thus, based upon the integral equations mentioned above, the conservation equations of mass, momentum, and energy can be derived using the net fluid volume and the available flow areas. Similar concept has also been used by Sha et al. [17] in the three-dimensional thermal hydrodynamic analysis of reactor components.

\section{The Above-Core Hydrodynamics}

In the analysis of HCDAs one important area that so far has not been rigorously considered is the above-core hydrodynamics induced by the Upper Internal Structure (UIS). The shape of the UbS generally differs among various reactors. In the Fast Flux Test Facility (FFTF), the upper internal structure consists of three instrument trees located above the core. In the clinch River Breeder Reactor (CRBR), the main body of the upper internal structure comprises of four support columns, an upper plate, and a lower plate holding numerous flow-guide passageways surrounded by the stagnant sodium as shown in Fig. 4. Since the passageways provide the only flow area for redirecting 
the core sodium axially into the outlet plenum, the presence of the upper internal structure definitely has the effect of confining and retarding the HCDA bubble as well as slowing down the upward motion of the fluid. As a result, the HCDA energy release, the slug-impact loading, and the responses of the structural components and containment can be significantly influenced by this structure. Thus, to provide a more complete analysis of primary-containment response, the treatment of the upper internal structure should be incorporated into the computer model.

Since at the present time the structural dynamic program for treating the mo-

tion of the UIS is not available, the main body of the UIS is considered to be rigid and fixed in the space. The flow-area reduction due to presence of the support columns is ignored in the nydrodynamic calculations. The assembly portion of the UIS is considered as $z$ yerforated structure. The governing difference equations will be described below.

\section{Fluid Motion Inside Coolant Passageways}

The flow inside the coolant passageways is viscous but one-dimensional. The mass and momentum equations are:

$$
\frac{\partial \rho}{\partial t}+\frac{\partial \rho \tilde{v}}{\partial z}=0
$$

and

$$
\frac{\partial \rho \tilde{v}}{\partial t}+\frac{\partial \rho \tilde{v}^{2}}{\partial z}=-\frac{\partial}{\partial z}(p+q)-\tilde{E} \frac{\rho \tilde{v}|\tilde{v}|}{2 D}
$$

where $\tilde{v}$ is the axial velocity, $f$ is the friction factor depending on the wall roughness and the Reynolds number, and $D$ is the average hydraulic diameter of the coolant passageways associated with each radial zone. The drag-force term in the momentum equation represents the friction retardation force acting on the coolant passageway wal1. The absolute-value sign indicates that the drag force always acts opposite to the direction of the axial velocity component. The viscous pressure $q$ is given by

$$
q=-\lambda \frac{\partial \tilde{v}}{\partial z} .
$$


The finite difference forms of the axial momentum equations can be easily derived from the integral equation by summing term by term contributions of the convective fluxes and the surface forces on the bounding surfaces of a control volume $A B C D$ shown in Fig. 5. The resulting equation is

$$
\begin{aligned}
\phi_{i} \frac{(\rho \tilde{v})_{i, j+\frac{1}{2}}^{n+1}-(\rho \tilde{v})_{i, j+\frac{1}{2}}^{n}}{\delta t} & =\phi_{i}\left[\frac{\psi}{\delta z}\left(\bar{P}_{i, j}-\bar{P}_{i, j+1}\right)+\frac{1-\psi}{\delta z}\left(p_{i, j}^{n}-p_{i, j+1}^{n}\right)\right] \\
& +s_{i, j+\frac{1}{2}}
\end{aligned}
$$

were

$$
s_{i, j+\frac{1}{2}}=\frac{\phi_{i}}{\delta z}\left\{\left[\left(\rho \tilde{v}^{2}\right)_{i, j}-\left(\rho \tilde{v}^{2}\right)_{i, j+1}\right]+q_{i, j}-q_{i, j+1}\right\}-f \frac{\rho \tilde{v}|\tilde{v}|}{2 D}
$$

The finite-difference mass equation becomes

$$
\frac{\rho_{i, j}^{n+1}-\rho_{i, j}^{n}}{\delta t}=\frac{\theta}{\delta z}\left[(\rho \tilde{v})_{i, j-\frac{1}{2}}^{n+1}-(\rho \tilde{v})_{i, j+\frac{1}{2}}^{n+1}\right]+\frac{1-\theta}{\delta z}\left[(\rho \tilde{v})_{i, j-\frac{1}{2}}^{n}-(\rho \tilde{v})_{i, j+\frac{1}{2}}^{n}\right] .
$$

The equation of state has the form

$$
\overline{\mathbf{P}}_{i, j}=P_{i, j}^{n}+c_{i, j}^{n}\left(\rho_{i, j}^{n+1}-\rho_{i, j}^{n}\right) \text {. }
$$

Thus, following the difference scheme of the implicit Eulerian hylrodynamics $[11,13,15]$, a modified Poisson equation governing the advanced-time pressures in the coolant passageways can be obtained by eliminating the advanced-time densities and velocities in Eq. (23). This results in

$$
\bar{P}_{i, j}\left[\frac{1}{c_{i, j}^{n}}+2 \theta \psi \frac{\delta t^{2}}{\delta z^{2}}\right]=H_{i, j}+\theta \psi \delta t^{2}\left[\frac{\bar{P}_{i, j-1}+\bar{P}_{i, j+1}}{\delta z^{2}}\right] \text {, }
$$

where

$$
\begin{aligned}
H_{i, j} & =\frac{p_{i, j}^{n}}{c_{i, j}^{n}}+\frac{\theta \delta t^{2}}{\delta z} \frac{1}{\phi_{i}}\left(s_{i, j-\frac{1}{2}}-s_{i, j+\frac{1}{2}}\right)+\frac{\delta t}{\delta z}\left[(\rho \tilde{v})_{i, j-\frac{1}{2}}^{n}-(\rho \tilde{v})_{i, j+\frac{1}{2}}^{n}\right] \\
& +\frac{\theta(1-\psi)}{\delta z^{2}}\left(P_{i, j-1}^{n}+P_{i, j+1}^{n}-2 P_{i, j}^{n}\right),
\end{aligned}
$$

is the source term evaluated from previous-cycle pressures and velocities. 


\section{Fluid Motion Above the Perforated Structure}

At the exit of the perforated structure the flow is two dimensional. The radial momentum equation can be derived using control volume ABCDEF shown in Fig. 5 . The upper boundary of the UIS is assumed to be a porous polygon. Thus, in the calculation of the surface forces and the convected fluxes we homogenize the perforated structure member so that the total mass flux through the coolant passageways is uniformly distributed over the entire cell boundary. In other words, for the case shown in Fig. 6 we let

$$
v_{i, j-\frac{1}{2}}=\phi_{i} \tilde{v}_{i, j-\frac{1}{2}}
$$

Adding up the convective fluxes and the surface forces with respect to the bounding surfaces shown in Fig. 6, the radial momentum equation has the form

$$
\begin{aligned}
\frac{(\rho u)_{i+\frac{1}{2}, j}^{n+1}+(\rho u)_{i+\frac{1}{2}, j}^{n}}{\delta t} & =\frac{\psi}{\delta r}\left(\bar{P}_{i, j}-\bar{P}_{i+1, j}\right)+\frac{1-\psi}{\delta r}\left(p_{i, j}^{n}-P_{i+1, j}^{n}\right) \\
& +R_{i+\frac{1}{2}, j},
\end{aligned}
$$

where

$$
\begin{aligned}
& R_{i+\frac{1}{2}, j}=\frac{1}{r_{i+\frac{1}{2}} \delta r}\left[r_{i}\left(\rho u^{2}\right)_{i, j} \cdots r_{i+1}\left(\rho u^{2}\right)_{i+1, j}\right] \\
& +\frac{1}{\delta z}\left\{\frac{1}{2 r_{i+\frac{1}{2}}}\left[\phi_{i} r_{i}(\rho u \tilde{v})_{i, j-\frac{1}{2}}+\phi_{i+1} r_{i+1}(\rho u \tilde{v})_{i+1, j-\frac{1}{2}}\right]\right. \\
& \left.-(\rho \mathrm{uv})_{i+\frac{1}{2}, j+\frac{1}{2}}\right\}+\frac{1}{\delta r}\left(q_{i, j}-q_{i+1, j}\right)+g_{r} \rho_{i+\frac{1}{2}, j} \\
& +\frac{\mu}{\delta z}\left[\frac{u_{i+\frac{1}{2}, j+1}-u_{i+\frac{1}{2}, j}}{\delta z}-\frac{v_{i+1, j+\frac{1}{2}}-v_{i, j+\frac{1}{2}}}{\delta r}\right. \\
& \left.-\frac{u_{i+\frac{1}{2}, j}-u_{i+\frac{1}{2}, j-1}}{\delta z}+\frac{\phi_{i+1} \tilde{v}_{i+1, j-\frac{1}{2}}-\phi_{i} \tilde{v}_{i, j-\frac{1}{2}}}{\delta r}\right] \text {. }
\end{aligned}
$$

The finite-difference representation of the axial momentum equation can be obtained from summation of the convective fluxes and the surface forces with respect to the control volume centering at $\left(i, j+\frac{1}{2}\right)$, and has the form 


$$
\begin{aligned}
\frac{(\rho v)_{i, j+\frac{1}{2}}^{n+1}-(\rho v)_{i, j+\frac{1}{2}}^{n}}{\delta t} & =\frac{\psi}{\delta z}\left(\bar{p}_{i, j}-\bar{p}_{i, j+1}\right)+\frac{1-\psi}{\delta z}\left(p_{i, j}^{n}-p_{i, j+1}^{n}\right) \\
& +s_{i, j+\frac{1}{2}},
\end{aligned}
$$

where

$$
\begin{aligned}
s_{i, j+\frac{1}{2}} & =\frac{1}{r_{i} \delta r}\left[(\rho u v r)_{i-\frac{1}{2}, j+\frac{1}{2}}-(\rho u v r)_{i+\frac{1}{2}, j+\frac{1}{2}}\right]+\frac{1}{\delta z}\left[v _ { i , j + \frac { 1 } { 2 } } \left(\rho_{i, j} v_{i, j-\frac{1}{2}}\right.\right. \\
& \left.\left.-\rho_{i, j+1} v_{i, j+3 / 2}\right)\right]+\frac{1}{\delta z}\left(q_{i, j}-q_{i, j+1}\right)+g_{z} \rho_{i, j+\frac{1}{2}} \\
& -\frac{\mu}{r_{i} \delta r}\left[r_{i+\frac{1}{2}}\left(\frac{u_{i+\frac{1}{2}, j+1}-u_{i+\frac{1}{2}, j}}{\delta z}-\frac{v_{i+1, j+\frac{1}{2}}-v_{i, j+\frac{1}{2}}}{\delta r}\right)\right. \\
& \left.-r_{i-\frac{1}{2}}\left(\frac{u_{i-\frac{1}{2}, j+1}-u_{i-\frac{1}{2}, j}}{\delta z}-\frac{v_{i, j+\frac{1}{2}}-v_{i-1, j+\frac{1}{2}}}{\delta r}\right)\right]
\end{aligned}
$$

The finite-difference mass equation for cell $(i, j)$ is

$$
\frac{\rho_{i, j}^{n+1}-\rho_{i, j}^{n}}{\delta t}=\theta D_{i, j}^{n+1}+(1-\theta) D_{i, j}^{n},
$$

where

$$
\begin{aligned}
D_{i, j}^{n} & =\frac{1}{r_{i} \delta r}\left[r_{i-\frac{1}{2}}(\rho u)_{i-\frac{1}{2}, j}^{n}-r_{i+\frac{1}{2}}(\rho u)_{i+\frac{1}{2}, j}^{n}\right] \\
& +\frac{1}{\delta z}\left[\phi_{i}(\rho \tilde{v})_{i, j-\frac{1}{2}}^{n}-(\rho v)_{i, j+\frac{1}{2}}^{n}\right],
\end{aligned}
$$

represents the volumetric dilatation of cell $(i, j)$ obtained from the previoustime cycle values, $D_{i, j}^{n+1}$ has exactly the same form but is evaluated at the $(n+1)-t h$ cycle.

The equation of state is

$$
\bar{P}_{i, j}=P_{i, j}+c_{i, j}^{n}\left(\rho_{i, j}^{n+1}-\rho_{i, j}^{n}\right) \text {. }
$$

Eliminating the advanced-time densities and velocities in Eq. (30) we have a modified Potsson equation governing the new pressure field above the perforated UIS, which has the form 


$$
\begin{aligned}
\overline{\mathrm{P}}_{j, j}\left[\frac{1}{c_{i, j}^{n}}\right. & \left.+2 \theta \psi \delta t^{2}\left(\frac{1}{\delta r^{2}}+\frac{1}{\delta z^{2}}\right)\right]=H_{i, j}+\theta \psi \delta t^{2}\left[\frac{r_{i-\frac{1}{z}} \bar{P}_{i-1, j}+r_{i+\frac{1}{2}} \bar{P}_{i+1, j}}{r_{i} \delta r^{2}}\right. \\
& \left.+\frac{\phi_{i} \bar{P}_{i, j-1}+\left(1-\phi_{i}\right) \bar{P}_{i, j}+\bar{P}_{i, j+1}}{\delta z^{2}}\right]
\end{aligned}
$$

where

$$
\begin{aligned}
H_{i, j} & =\frac{P_{i, j}}{c_{i, j}}+\frac{\theta \delta t}{r_{i}} \frac{5 r}{\delta r}\left\{\frac{1-\psi}{\delta r}\left[r_{i-\frac{1}{2}}\left(P_{i-1, j}-P_{i, j}\right)-r_{i+\frac{1}{2}}\left(P_{i, j}-P_{i+1, j}\right)\right]\right. \\
& \left.+r_{i-\frac{1}{2}} R_{i-1_{i}, j}-r_{i+l_{2}} R_{i+\frac{1}{2}, j}\right\}+\frac{\partial \delta t^{2}}{\partial z}\left\{\frac { 1 - \psi } { \delta z } \left[p_{i}\left(P_{i, j-1}-P_{i, j}\right)\right.\right. \\
& \left.\left.\left.-\left(1-\phi_{i}\right) g_{z} \delta z i\right)_{i, j}-\left(P_{i, j}-P_{i, j+1}\right)\right]+s_{i, j-\frac{1}{2}}-s_{i, j+\frac{1}{2}}\right\} \\
& +\delta t D_{i, j}^{n} .
\end{aligned}
$$

\section{Fluid Mocion Below the Perforated Structure}

Below the perforated structure the flow is also two-dimensional. Here, we make the same assumption that the state of stress is continuous throughout the fluid element enclosed by the respective control volume for derivations of the momentum equations. Thus, similar to the formulation of difference equations above the perforated structure we assume that the coolant passageways are uniformly dispersed over the upper cell boundary. Thus, we have

$$
\hat{\mathrm{v}}_{i, j+\frac{1}{2}}=\phi_{i} \tilde{v}_{i, j+\frac{1}{2}} \text {. }
$$

Similar to the development of equations for flows above the perforated UIS, the radial momentum equation becomes

$$
\begin{aligned}
\frac{(\rho u)_{i+\frac{1}{2}, j}^{n+1}-(\rho u)_{i+\frac{1}{2}, j}^{n}}{\delta t} & =\frac{\psi}{\delta r}\left(\bar{P}_{i, j}-\bar{P}_{i+1, j}\right)+\frac{1-\psi}{\delta r}\left(P_{i, j}^{n}-P_{i+1, j}^{n}\right) \\
& +R_{i+\frac{1}{2}, j},
\end{aligned}
$$

where 


$$
\begin{aligned}
R_{i+\frac{1}{2}, j} & =\frac{1}{r_{i+\frac{1}{2}} \delta r}\left[r_{i}\left(\rho u^{2}\right)_{i, j}-r_{i+1}\left(\rho u^{2}\right)_{i+1, j}\right]+\frac{1}{\delta z}\left\{(\rho u v)_{i+\frac{1}{2}, j-\frac{1}{2}}\right. \\
& \left.-\frac{1}{2 r_{i+\frac{1}{2}}}\left[\phi_{i} r_{i}(\rho u \tilde{v})_{i, j+\frac{1}{2}}+\phi_{i+1} r_{i+1}(\rho u v)_{i+1, j+\frac{1}{2}}\right]\right\} \\
& +\frac{1}{\delta r}\left(q_{i, j}-q_{i+1, j}\right)+g_{r} \rho_{i+\frac{1}{2}, j}+\frac{\mu}{\delta z}\left[\frac{u_{i+\frac{1}{2}, j+1}-u_{i+\frac{1}{2}, j}}{\delta z}\right. \\
& \left.-\frac{\hat{v}_{i+1, j+\frac{1}{2}}-\hat{v}_{i, j+\frac{1}{2}}}{\delta r}-\frac{u_{i+\frac{1}{2}, j}-u_{i+\frac{1}{2}, j-1}}{\delta z}+\frac{v_{i+1, j-\frac{1}{2}}-v_{i, j-\frac{1}{2}}}{\delta r}\right],
\end{aligned}
$$

is the source term with the form of $\hat{v}$ defined in Eq. (33).

The finite-difference representation of the axial momentum equation is

$$
\begin{aligned}
\phi_{i} \frac{(\rho \tilde{v})_{i, j+\frac{1}{2}}^{n+1}-(\rho \tilde{v})_{i, j+l_{i}}^{n}}{\delta t} & =\phi_{i}\left[\frac{\psi}{\delta z}\left(\bar{P}_{i, j}-\bar{P}_{i, j+1}\right)+\frac{1-\psi}{\delta z}\left(P_{i, j}^{n}-P_{i, j+1}\right)\right] \\
& +s_{i, j+\frac{1}{2}}
\end{aligned}
$$

where

$$
\begin{aligned}
& s_{i, j+\frac{1}{2}}=\frac{\phi_{i}}{\delta z}\left[\left(\rho \tilde{v}^{2}\right)_{i, j}-\left(\rho \tilde{v}^{2}\right)_{i, j+1}\right]+\frac{1}{\delta z}\left(q_{i, j-1}-q_{i, j}\right) \\
& +\phi_{i} \frac{\rho_{j, j+1}+\rho_{i, j}}{2} q_{z}+\left(1-\phi_{i}\right) \rho_{i, j} q_{z} \\
& -\frac{\mu}{r_{i} \delta r}\left[r_{i+\frac{1}{2}}\left(\frac{u_{i+\frac{1}{2}, j+1}-u_{i+\frac{1}{2}, j}}{\delta z}-\frac{\hat{v}_{i+1, j+\frac{1}{2}}-\hat{v}_{i, j+\frac{1}{2}}}{\delta r}\right)\right. \\
& \left.-r_{i-\frac{1}{2}}\left(\frac{u_{i-\frac{1}{2}, j+1}-u_{i-\frac{1}{2}, j}}{\partial z}-\frac{\hat{v}_{i, j+\frac{1}{2}}-\hat{v}_{i-1, j+\frac{1}{2}}}{\delta r}\right)\right] \text {, }
\end{aligned}
$$

is the source term, $\hat{v}$ again has the same form as of Eq. (33).

Similar to the momentum equations a weighting constant $\theta$ varying from 0.0 to 1.0 can be used for the finite-difference mass equation to indicate relative level between purely explicit and purely implicit forms. This yields

$$
\frac{\rho_{1, j}^{n+1}-\rho_{i, j}^{n}}{\delta t}=\theta D_{i, j}^{n+1}+(1-\theta) D_{i, j}^{n} \text {, }
$$


where

$$
\begin{aligned}
D_{i, j} & =\frac{1}{r_{i} \delta r}\left[r_{i-\frac{1}{2}}(\rho u)_{i-\frac{1}{2}, j}-r_{i+\frac{1}{2}}(\rho u)_{i+\frac{1}{2}, j}\right] \\
& +\frac{1}{\delta z}\left[(\rho \tilde{v})_{i, j-\frac{1}{2}}-\phi_{i}(\rho \tilde{v})_{i, j+\frac{1}{2}}\right],
\end{aligned}
$$

again denotes the volumetric dilatation of cell $(i, j)$ with time index $n$ and $\mathrm{n}+1$ neglected in the expression.

Eliminating the advanced-time densities and velocities in the mass equation a modified Poisson equation characterizing the flow below the perforated structire is

$$
\begin{aligned}
\overline{\mathrm{P}}_{i, j}\left[\frac{1}{c_{i, j}^{n}}\right. & \left.+20 \psi \delta t^{2}\left(\frac{1}{\delta r^{2}}+\frac{1}{\delta z^{2}}\right)\right]=H_{i, j}+\theta \psi \delta t^{2}\left[\frac{r_{i-\frac{1}{2}} \overline{\mathrm{P}}_{i-1, j}+r_{i+j_{j}} \overline{\mathrm{P}}_{i+1, j}}{r_{i} \delta t^{2}}\right. \\
& \left.+\frac{\overline{\mathrm{P}}_{i=j-1}+\phi_{i} \overline{\mathrm{P}}_{i, j+1}+\left(1-\phi_{i}\right) \overline{\mathrm{P}}_{i, j}}{\delta z^{2}}\right]
\end{aligned}
$$

in which

$$
\begin{aligned}
H_{i, j} & =\frac{P_{1, j}}{c_{i, j}}+\frac{\theta \delta \frac{t^{2}}{r_{i}}}{\delta r}\left\{\frac { 1 - \psi } { \delta r } \left[r_{i-\frac{1}{2}}\left(P_{i-1, j}-P_{i, j}\right)-r_{i+\frac{1}{2}}\left(\underline{p}_{i, j}-P_{i+1, j}:\right]\right.\right. \\
& \left.+r_{i-\frac{1}{2}} R_{i-\frac{1}{2}, j}-r_{i+\frac{1}{2}} R_{i+\frac{1}{2}, j}\right\}+\frac{\theta \delta t^{2}}{\delta z}\left\{\frac { 1 - \psi } { \delta z } \left[\left(P_{i, j-1}-P_{i, j}\right)\right.\right. \\
& \left.\left.-\phi_{i}\left(P_{i, j}-P_{i, j+1}\right)-\left(1-\phi_{i}\right) \rho_{i, j} g_{z} \delta_{z}\right]+s_{i, j-\frac{1}{2}}-s_{i, j+\frac{1}{2}}\right\} \\
& +\delta t D_{i, j}^{n},
\end{aligned}
$$

is the source term; $D_{i, j}^{n}$ is defined in Eq. (39). Also, in calsulating $H_{i, j}$ the momentum sources $R_{i-\frac{1}{2}, j}$ and $s_{i, j-\frac{1}{2}}$ are also needed. The term $R_{i-\frac{1}{2}, j}$ can be calculated from Eq. (35) by replacing index $i$ with $i-1$; whereas $S_{i, j-\frac{1}{2}}$ can be evaluated from Eq. (9) of the ICE technique by changing $j$ with $j-1$. 


\section{Moving Cover and Sodium Spillage}

The Eulerian hydrodynamics is ideal for long-duration calculations for problems involving large material distortions. The basic hydrodynamic technique has an option for treating inflow and outflow boundary conditions. However, the size of the wall opening in the basic ICE method is limited to multiples of the cell size. In other words, if the wall opening is small, the Eulerian cell dimension must also be made small. In addition, the size of the opening in the basic hydrodynamic technique must remain constant. Thus, the opening does not vary with time, which is different from a gap generated by the slug-impact force on the reactor head cover at the head-vessel junction. Therefore, the basic hydrodynamic technique must be expanded in order to treat the coolant spillage problem more realistically.

In the Eulerian-Hydrodynamic analysis when the vessel head moves upward a set of irregular cells whose size varies in the axial direction according to the head displacement, is introduced on the top of the full-zone regular cell as shown in Fig. 7. Since in these irregular cells only the axial dimension of the cell varies, but the shape remains rectangular, it is possible to derive the governing difference equations with respect to these irregular cells. Several years ago, Chu [18] developed a quasi-Eulerian method which was used in conjunction with the ICECO code to study the effect of sodium spillage on slug impact. In that model the viscous stress tensor was neglected in the formulation, and a complete Donor-cell differencing scheme was utilized for evaluations of the source terms in the momentum equations and Poisson's equation.

Recently, the sodium-spillage model has been considerably revised and its scope of applicability is extended. The present mode1 (1) provides a more compartible formulation so that in certain circumstances the equations can be directly reduced to those used in the Eulerian technique $[11,13]$, (2) incorporates the correct, full viscous stress tensor, instead of a simple artificial viscosity, (3) introduces the option of using ZIP differencing scheme for calculating various source terms, and (4) includes provisions of computing coolant spillage through different leak paths. 
Here, the control-volume technique is employed, in which the conservation equations are generalized iu take into account the penetration holes and the side opening. The upper boundaries of the irregular cells are considered as inviscid, moving with the same velocity as the cover head. Thus, separation of the entire coolant-free surface with the reactor cover is not permitted after slug impact.

In the analysis, two fictitious cells, one on the top and one at the bottom of the irregular cell, are introduced for convenience of calculation (see Fig. 7). Fluid variables in the fictitious cells of both the irregular and regular cells are obtained from interpolation or extrapolation. To simplify the anal-ysis we assume that (1) the penetration is located on the top center of the irregular cell for convenience of moving particles inside the top opening, (2) only one penetration is allowed for each cell, (3) the penetration is considered to open when the impulse on this penetration area exceeds a certain input value.

Thus, similar to the derivation of difference equations for the above-core hydrodynamics the governing difference equations near the reactor cover are formulated by summing up term by term contributions of the integral Eqs. (16-18). The radial momentum equation can be derived using control volume ABCDEF shown in Fig. 8. Again to ensure the stress continuity for calculating the viscous stress tensor it is necessary to distribute the velocity in the penetration opening uniformly throughout the upper cell boundary. For the case shown in Fig. 8, we let

$$
\hat{v}_{i, j+\frac{1}{2}}=n_{i}\left(v_{0}\right)_{i, j+\frac{1}{2}}+\left(1-n_{i}\right) v_{i, j+\frac{i}{2}}
$$

where $\hat{v}$ is the mean value of the axial velocity component; $n_{i}$ is the ratio of the penetration-opening area to the area of upper cell boundary; $v_{0}$ is the fluid velocity at the penetration o, "ning; $v$ is the velocity of the moving cover. For convenience, we further define

$$
\alpha_{i}=0.5 n_{i} r_{i} / r_{i+\frac{l}{2}} \text { and } \beta_{i+1}=0.5 n_{i+1} r_{i+1} / r_{i+\frac{1}{2}} \text {. }
$$

Thus, based on a control volume ABCDEF shown in Fig. 8, the radial momentum equation relative to the right-hand cell boundary has the form 


$$
\begin{aligned}
\frac{(\rho u)_{i+1 / 2, j}^{n+1}-(\rho u)_{i+\frac{1}{2}, j}^{n}}{\delta t} & =\frac{\psi}{\delta r}\left(\bar{P}_{i-1, j}-\bar{P}_{i, j}\right)+\frac{1-\psi}{\delta r}\left(P_{i-1, j}^{n}-P_{i, j}^{n}\right) \\
& +R_{i+\frac{1}{2}, j},
\end{aligned}
$$

in which

$$
\begin{aligned}
R_{i+\frac{1}{2}, j} & =\frac{1}{r_{i+\frac{1}{2}}}{ }\left[r_{i}\left(\rho u^{2}\right)_{i, j}-\left(\rho u^{2}\right)_{i+1, j}\right]+\frac{1}{\delta y}\left[(\rho u v)_{i+\frac{1}{2}, j-\frac{1}{2}}\right. \\
& -\alpha_{i}\left(\rho u v_{0}\right)_{i, j+\frac{1}{2}}-\beta_{i+1}\left(\rho u v_{0}\right)_{i+1, j+\frac{1}{2}} \\
& \left.-\left(1-\alpha_{i}-\beta_{i+1}\right)(\rho u v)_{j+\frac{1}{2}, j+\frac{1}{2}}\right]+\frac{1}{\delta r}\left(q_{i, j}-q_{i+1, j}\right)+g_{r} \rho_{i+\frac{1}{2}, j} \\
& +\frac{\mu}{\delta y}\left[\frac{u_{i+\frac{1}{2}, j+1}-u_{i+\frac{1}{2}, j}}{\delta z}-\frac{v_{i+1, j+\frac{1}{2}}-\hat{v}_{i, j+\frac{1}{2}}}{\delta r}\right. \\
& \left.-\frac{u_{i+\frac{1}{2}, j}-u_{i+\frac{1}{2}, j-1}}{\delta z}+\frac{\left.v_{i+1, j-\frac{1}{2}}-v_{i, j-\frac{1}{2}}\right]}{\delta r}\right]
\end{aligned}
$$

is the source term with $\hat{v}, \alpha$ and $\beta$ defined in Eqs. (42) and (43), respectively. Note that $R_{i+\frac{1}{2}, j}$ is the source term, which has exactly the same form as the ICE technique in the case of full-cell openings (i.e., $n_{i}=n_{i+1}=1$ ).

Likewise, the finite-difference representation of the axial momentum equation relative to the upper cell boundary $\left(i, j+\frac{1}{2}\right)$ can be obtained using the control volume shown in Fig. 9, and has the form

$$
\begin{aligned}
n_{i} \frac{\left(\rho v_{0}\right)_{i, j+\frac{1}{2}}^{n+1}-\left(\rho v_{0}\right)_{i, j+\frac{1}{2}}^{n}}{\delta t} & +\left(1-n_{i}\right) \frac{(\rho v)_{i, j+\frac{1}{2}}^{n+1}-(\rho v)_{i, j+\frac{1}{2}}^{n}}{\delta t} \\
& =\frac{\eta_{i}}{d y}\left[\psi\left(\bar{P}_{i, j}-\bar{P}_{o_{i, j+1}}\right)+(1-\psi)\left(P_{i, j}^{n}-P_{o}^{n}\right)\right] \\
& +\frac{1-\eta_{i, j+1}}{d y}\left[\psi\left(\bar{P}_{i, j}-\bar{P}_{i, j+1}\right)+(1-\psi)\left(P_{i, j}^{n}-P_{i, j+1}^{n}\right)\right] \\
& +s_{i, j+\frac{1}{2}},
\end{aligned}
$$

where 


$$
\begin{aligned}
s_{i, j+\frac{1}{2}} & =\frac{n_{i}}{d y}\left[\left(\rho v_{0}^{2}\right)_{i, j}-\left(\rho v_{0}^{2}\right)_{i, j+1}\right]+\frac{1-n_{i}}{d y}\left[\left(\rho v^{2}\right)_{i, j}-\left(\rho v^{2}\right)_{i, j+1}\right] \\
& +\frac{1}{r_{i} \delta r}\left[r_{i-\frac{1}{2}}(\rho u v)_{i-\frac{1}{2}, j+\frac{1}{2}}-r_{i+\frac{1}{2}}(\rho u v)_{i+\frac{1}{2}, j+\frac{1}{2}}\right]+\frac{1}{\delta y}\left(q_{i, j}-q_{i, j+1}\right) \\
& \left.+n_{i} \frac{\rho_{i, j+1}+\rho_{i, j}}{2} g_{r}+\left(1-n_{i}\right) \frac{\rho_{i, j+1}+\rho_{i, j}}{2}-\hat{v}_{i+1, j+\frac{1}{2}}-\hat{v}_{i, j+\frac{1}{2}}\right) \\
& -\frac{\mu r}{r_{i} \delta r}\left[r_{i+\frac{1}{2}}\left(\frac{u_{i+\frac{1}{2}, j+1}-u_{i+\frac{1}{2}, j}}{\delta y}-\frac{\hat{i}_{i}}{\delta r}-\hat{v}_{i, j+\frac{1}{2}}-\hat{v}_{i-1, j+\frac{1}{2}}\right)\right] \\
& -r_{i-\frac{1}{2}}\left(\frac{u_{i-\frac{1}{2}, j+1}-u_{i-\frac{1}{2}, j}-\frac{v_{r}}{\delta r}}{}\right.
\end{aligned}
$$

is the source term, $\rho_{0}$ is the density outside the containment, dy is the axial size of the irregular cells, $\tilde{v}$ is again the mean value of the axial velocity component similar to the one expressed in Eq. (42); also, $\overline{\mathrm{p}}_{0}$ and $\mathrm{P}_{0}^{\mathrm{n}}$ are the pressures outside the opening at the $(n+1)$ th and nth cycle, respectively.

Eliminating all the advanced-time densities and velocities in the mass equation similar to the scheme developed by Chu [18] a modified Poisson equation for the irregular cells, including the effects of penetration hole or side opening, is of the form

$$
\begin{aligned}
\bar{P}_{i, j}\left[\frac{1}{c_{i, j}^{n}}\right. & \left.+2 \theta \psi \delta t^{2}\left(\frac{1}{\delta r^{2}}+\frac{1}{\delta y^{2}}\right)\right]=H_{i, j}+\theta \psi \delta t^{2}\left[\frac{r_{i-\frac{1}{2}} \bar{P}_{i-1, j}+r_{i+\frac{1}{2}} \bar{P}_{i+1, j}}{r_{i} \delta r^{2}}\right. \\
& \left.+\frac{\bar{P}_{i, j-1}+n_{i} \bar{P}_{o_{i, j+1}}+\left(1-n_{i}\right) \bar{P}_{i, j+1}}{\delta y^{2}}\right]
\end{aligned}
$$

in which

$$
\begin{aligned}
H_{i, j} & =\frac{P_{i, j}}{c_{i, j}}+\frac{\theta \delta t^{2}}{r_{i} \delta r}\left\{\frac{1-\psi}{\delta r}\left[r_{i-\frac{1}{2}}\left(P_{i-1, j}-P_{i, j}\right)-r_{i+\frac{1}{2}}\left(P_{i, j}-P_{i+1, j}\right)\right]\right. \\
& \left.+r_{i-\frac{1}{2}} R_{i-\frac{1}{2}}-r_{i+\frac{1}{2}} R_{i+\frac{1}{2}}\right\}+\frac{\theta \delta t^{2}}{\delta y}\left\{\frac { 1 - \psi } { \delta y } \left[\left(P_{i, j-1}-P_{i, j}\right)\right.\right. \\
& \left.\left.-n_{i}\left(P_{i, j}-P_{o_{i, j+1}}\right)-\left(1-n_{i}\right)\left(P_{i, j}-P_{i, j+1}\right)\right]+s_{i, j-\frac{1}{2}}-s_{i, j+\frac{1}{2}}\right\} \\
& +\delta t D_{i, j}^{n},
\end{aligned}
$$


is the source term of the Poisson equation, $D_{i, j}^{n}$ is the volumetric dilatation [18] with all quantities evaluated at previous cycle $n$.

\section{E. Flow Near Primary Vessel and Deformable Internals}

The mathematical model for treating fluid-structure interactions near the primary vessel and deformable internals was described in ICECO-II report [13]. Here, we briefly mention the numerical treatment.

\section{The Fluid-Vessel Interface}

The interface between the fluid and the deformable vessel (such as the primary vessel, pipe wall, etc.) is treated as a free surface of the fluid but: subjected to a pressure distribution that determines its motion. Such interfacial motion is made to conform with the motion of the structure generated by the internal hydrodynamic pressure loading. Since the viscosity of the fluid (like water or sodium) is very small, the sliding boundary condition is used at the interface which requires that the fluid is allowed to slide along the interface, but in the normal direction, the fluid and the structure are forced to nove together.

A convenient way to satisfy the sliding boundary condition is by iteratively adjusting the pressure on the fluid interface by an amount proportional to the mass flow rate of the fluid normal to the boundary of the deformable structure. Thus, denoting $h$ and $h+1$ the previous and new iterates, respectively, the relaxation equation for the pressure in the fluid-structure interfacial cell $(k, \ell)$ is $[13,19]$

$$
\overline{\mathrm{p}}_{\mathrm{k}, \ell}^{\mathrm{h}+1}=\overline{\mathrm{p}}_{\mathrm{k}, \ell}^{\mathrm{h}}-\frac{\rho \delta}{2 \delta t}\left\{\left[\overline{\mathrm{v}}_{\mathrm{p}}-\overline{\mathrm{v}}_{\mathrm{b}}\right] \cdot \overline{\mathrm{n}}\right\}_{\mathrm{k}, \ell} \text {. }
$$

In the above equation, $\bar{v}_{b}$ is the velocity vector at the midpoint of the structure segment, $\bar{v}_{p}$ is the velocity vector of the fluid particle, $\bar{n}$ is the unit normal of the boundary pointing into the fluid, and $\delta$ is the smallest value between $\delta \mathrm{r}$ and $\delta z$. Thus, during the pressure iteration, one can see that if the particles attempt to cross the boundary the pressure in the boundary cell will be increased, forcing fluid to flow away from the boundary and vice versa. 


\section{Interaction of Fluids with Deformable Internals}

Since fluids have different motions on both sides of the internal structure, the field variables such as $p, p, u$, and $v$ in the cell containing structure are assumed to have different values. For instance, if the $\operatorname{cell}(i, j)$ contains the core barrel where both sides are submerged in the fluid the field variables should have two sets of values. The variables $\mathrm{P}^{\ell}, \mathrm{p}^{\ell}, \mathrm{u}^{\ell}$, and $\mathrm{v}^{\ell}$ are calculated in such a way that the fluid on the left-hand side of the core barrel slides tangentially. Likewise, $\mathrm{p}^{\mathrm{r}}, \mathrm{\rho}^{\mathrm{r}}, \mathrm{u}^{\mathrm{r}}$, and $\mathrm{v}^{\mathrm{r}}$ are obtained so that the fluid on its right-hand side also moves tangentially. Similarly, field variables can be obtained for the fluid located at the top or bottom of the structure. In general, for those variables at the exterior side of a Eulerian cell which cannot be computed by the conservation equation, extrapolation of their values from the interior region is necessary. The velocity components can be obtained from the mass conservation equation. These multivalued velocity fields will be used consistently in the particle-movement and source-term modifications. They are also utilized in handling fluid motion at the geometrical discontinuities. For further details, see Ref. [13].

Analysis of fluid-structure interactions involving flexible internals is a complex problem. The analysis is further complicated if the structure possesses cerlain perforated openings, like the core-support-structure shown in Fig. 10. One way of dealing with this problem is to use the control-volume technique to derive the conservation equations and their difference representation with respect to the partial cell which is actually occupied by the fluid (such as CDEF shown in Fig. 10). However, because the structure component in general moves two dimensionally and the shape of the partial cell is highly irregular, the procedure of the control-volume technique is too cumbersome.

In order to accommodate the deformable internal and the perforated structures, we provide a scheme which still uses the relaxation equation to obtain the pressure adjacent to the structure but modifies that equation to account for the sodium flow through the openings. The basic idea is to homogenize the perforated structure member so that the total mass flux through the openings Is uniformly distributed throughout the entire member. In other words, for those members containing openings, such as $A B$ and $C D$ in Fig. 10, we allow 
fluid penetration and separation at the boundaries. To simplify the analysis, we further propose that: (a) fluids inside the coolant passage move one dimensionally, (b) sodium flows perpendicularly to the boundary at the entrance or exit, (c) the density variation inside the coolant passage is negligible, so that the amount of mass flowing into the passage is equal to the mass flowing out of the passage.

Thus, similar to Eq. (50) the modified relaxation equarion for $\operatorname{cell}(k, l)$ which contains of the boundary of an internal with or without perforated openings is

$$
\bar{p}_{k, \ell}^{h+1}=\bar{p}_{k, l}^{h}-\frac{\delta}{2 \delta t}\left\{\rho\left(\bar{v}_{p}-\bar{v}_{b}\right) \cdot \bar{n}-\rho \phi \tilde{v}\right\}_{k, l} .
$$

We recall that $\phi$ is the perforation ratio of the structural component, $\tilde{v}$ is the fluid velocity inside the coolant passageways and is evaluated from pressures on both sides of the structure [13].

\section{F. Treatment of the Bubble-Coolant Interface and Free Surface}

1. Marker Particle and Volume Fraction Methods.

In calculating the high-energy excursion it is necessary to know the positions of the bubble-coolant interface and the coolant free surface so that pressures applied to the fluid boundaries can be calculated. Two different approaches are commonly used in the Eulerian hydrodynamics for calculating the positions of the fluid boundaries. These two approaches generally referred as the markerparticle method [20] and the volume-fraction method [21]. The marker-particle method uses Lagrangian particles to define the free surface and the interface. These particles, initially embedded in the fluid, are advected to their new positions using a simple kinematic relation between the fluid velocities in the local Eulerian cells. Consequently, fluid boundaries are clearly defined and good spatial resolution can be achieved. Since curved surface is allowed within one Eulerian cell this approach is potentially accurate regardless whether coarse or fine mesh is chosen in the analysis.

The volume-fraction method, on the other hand, utilizes fluid-volume fraction to determine the interface position and orientation. Normally, the free surface 
within one Eulerian cell is considered as a straight line. Since no curvature of fluid boundary is allowed in the surface cell, position of free surface thus cannot be defined exactly. This approach is the equivalent of two marker particles per cell and generally works well if very fine mesh configurations are used in the analysis, but fails to provide good resolution if coarse mesh configuration is chosen.

Because of the difference mentioned above, the free surface is delineated by marker particles that move through the stationary network of cells. Each Eulerian cell is flagged to denote whether it is an empty cell (E) containing no fluid; a surface cell (S), which contains fluid but is next to an empty cell; or a full cell (F), which contains fluid and is not next to an empty cell.

\section{Free-Surface Boundary Conditions}

The correct free-surface boundary conditions are the continuities of the normal and tangential stresses suggested by Nichols and Hirt [20]. In general, for a viscous, compressible fluid the stress tensor is

$$
\sigma_{i j}=-p \delta_{i j}+\mu\left(\frac{\partial u_{i}}{\partial x_{j}}+\frac{\partial u_{j}}{\partial x_{i}}-2 / 3 \delta_{i j} \frac{\partial u_{\ell}}{\partial x_{\ell}}\right)+\xi \delta_{i j} \frac{\partial u_{\ell}}{\partial x_{\ell}},
$$

where $\delta_{i j}$ is the Kronecker delta, $\xi$ is another second coefficient of viscosity and is defined as $\xi=\lambda+2 / 3 \mu$.

Since most liquids the compressibilities are small, they may be regarded as practically incompressible. Thus, the stress tensor takes the form

$$
\sigma_{i j}=-p \delta_{i j}+\mu\left(\frac{\partial u_{i}}{\partial x_{j}}+\frac{\partial u_{j}}{\partial x_{i}}\right) \text {. }
$$

Considering a free surface with a unit area $\mathrm{n}$ as illustrated in Fig. 6, it can be shown that, in terms of the stress tensor, the force acting on this surface is

$$
\hat{F}_{S}=\hat{F}_{r}+\hat{F}_{z}
$$


where

$$
\hat{F}_{r}=n_{r}\left(\sigma_{r r} \hat{e}_{r}+\sigma_{r z} \hat{e}_{z}\right)
$$

and

$$
\hat{F}_{z}=n_{z}\left(\sigma_{r z} \hat{e}_{r}+\sigma_{z z} \hat{e}_{z}\right)
$$

Where $\hat{F}_{r}$ and $\hat{F}_{2}$ represent the force components acting on surfaces $\mathrm{AC}$ and $\mathrm{BC}$ which have outnormals parallel to the $r$ - and $z$-axis, respectively.

Neglecting the viscosity of the gas the condition of normal force continuity yields

$$
p-2 \mu\left[n_{r}^{2} \frac{\partial u}{\partial r}+n_{r} n_{z}\left(\frac{\partial u}{\partial z}+\frac{\partial v}{\partial r}\right)+n_{z}^{2} \frac{\partial v}{\partial z}\right]=p_{a},
$$

where $p_{a}$ is the pressure applied at the free surface; $n_{r}$ and $n_{z}$ are the $r$ and $z$ components of the unit outward normal vector to the surface $A B$.

Similarly, the vanishing of the shear force on surface $A B$ takes the form

$$
2 \mu\left[2 n_{r} m_{r} \frac{\partial u}{\partial r}+\left(n_{r} m_{z}+n_{z} m_{r}\right)\left(\frac{\partial u}{\partial z}+\frac{\partial v}{\partial r}\right)+2 n_{z} m_{z} \frac{\partial v}{\partial z}\right]=0,
$$

where $m_{r}$ and $m_{z}$ are the components of the unit tangential vector.

For problems involving shock-wave propagation the effect of viscosity, and hence of the boundary layer, is very small. The condition of normal force continuity at the free surface and the bubble-fluid interface implies that

$$
p_{i, j}=p_{a}
$$

Also, if the curvature of the free surface is small, the condition of vanishing of tangential force is

$$
\mu\left(\frac{\partial u_{n}}{\partial m}+\frac{\partial u_{m}}{\partial n}\right)=0
$$

$\because i \in r e \mathrm{n}$ denotes the outward normal direction and $\mathrm{m}$ the tangential direction to $\therefore$ : 10 cal free surface. In the two-dimensional flow if the surface is nearly $\because$ isutal or vertical, the condition is 


$$
\frac{\partial u}{\partial z}+\frac{\partial v}{\partial r}=0
$$

For the case shown in Fig. 11 , the radial velocity $u_{i+1 / 2, j+1}$ is calculated from the finite-difference expression of the above expression:

$$
u_{i+1 / 2, j+1}=u_{i+1 / 2, j}-\frac{\delta z}{\delta r}\left(v_{i+1, j+1 / 2}-v_{i, j+1 / 2}\right) \text {. }
$$

\section{G. Wave Transient in Cavitated Liquids}

During an HCDA pressure wave eminating from the reactor core encounters not only the surrounding structure, but also the coolant free surface from which it is reflected as a rarefaction wave. As the rarefaction wave propagates downward a tension regime is established over a large portion of the coolant domain. When a region of coolant passes into tension exceeciing its tensile strength it is found from experiments that a rapid growth of bubbles is observed. These bubbles contain vapor and gas if any was previously dissolved in the liquid and are presumed to have previously existed as invisible "cavitation nucli" distributed throughout the liquid. Thus, in order to provide an accurate analysis treatment of liquid cavitation must be considered.

Most hydrodynamic codes employ an equation of state which supplies a relationship between the pressure, density, and specific internal energy of the coolant. This equation of state together with conservation equations of mass, momentum, and energy permit the solution of the dynamic system. Compression equations of state have been determined for most liquids but very little is known about the equation of state of liquids in tension. One common approach for treating cavitation is analytically continuing the compressive equation of state into the tension regime and assuming a pressure cutoff at some point corresponding to the "tensile strength" of the liquid. Once the pressure cutoff is reached, variations in liquid volume (or density) do not affect the pressure, which remains at the cutoff value until the liquid recompacts. This model is usually referred to as the " $\mathrm{p}_{\text {min }}$ model" [22-24]. The typical pressure-volume and pressure-density curves for the " $\mathrm{p}_{\text {min }}$ model" are shown in Fig. 12.

Note that the " $\mathrm{p}_{\min }$ " model is the most simplest approach in which energy dissipations generated by the growth and collapse of the cavitated liquids are 
neglected. Since no damping or dissipation involved in the analysis, numerical calculations employing the explicit solution algorithm thus have spurious oscillations.

Another approach considering damping term suggests that energy dissipation can occur in a cavitated liquid as a result of the finite rate at which cavitation bubbles grow and collapse. When this effect is taken into account the pressurevolume ( $p-v)$ paths for cavitated liquids become a hysteresis loop rather than the open curve in connection with the " $p_{\min }$ " model. Thus, damping can be expected which could produce smoother pressure histories during recompaction of the cavitated liquid.

In this sophisticated cavitation model the dynamics of bubble growth is governed by the Rayleigh equation. Including surface tension and velocity damping this equation has the form

$$
R \frac{d^{2} R}{d t^{2}}+2 / 3\left(\frac{d R}{d t}\right)^{2}=-\frac{1}{\rho}\left(p+\frac{2 \sigma}{R}+4 \frac{\dot{d}}{R}\right),
$$

where $R$ is the average radius of the cavitated bubbles, i i'; the liquid density, $p$ is the pressure, $\sigma$ is the surface tension, $\forall$ is the viscosity.

Thus, if one attempts to include the bubble-growth rate in the numerical calculation, one must solve the Rayle:igh equation along with conservation equations. This could further complicate the analysis. Nevertheless, this seems to be the only way to improve the solution of the explicit hydrodynamic codes.

Since the ICECO code uses the implicit integration scheme, the solution is stable and the adverse effect of fluid cavitation on the numerical results is less severe than those of the explicit containment codes. Therefore, the

equation of state similar to the " $p_{\text {min }}$ " model shown in Fig. 12 has been incorporated into ICECO for calculating wave transient in the cavitated liquid.

\section{H. Heat Transfer and Fuel-Coolant Interaction}

Several years ago a fuel-coolant interaction (FCI) model [12] was incorporated into the ICECO code to study various heat transfer problems related to the 
containment analysis. This model is the quasi-steady-state model (QSS) Ref. [25] in which the only resistance to heat transfer is assumed as due to the thermal conductivity of the fuel. Since the thermal conductivity of the liquid sodium is higher by an order of magnitude than that of the fuel, it is reasonable to neglect the thermal resistance due to sodium. The heat-transfer rate for the QSS model is given by the relation

$$
\dot{q}=\mathrm{hA}\left(\mathrm{T}_{\mathrm{f}}-\mathrm{T}\right) \text {, }
$$

where

$$
\begin{aligned}
& \dot{q}=\text { heat-transfer rate, } \\
& T_{f}=\text { fuel temperature, } \\
& T=\text { sodium-coolant temperature, }
\end{aligned}
$$

and $h A$ is a parametric heat-transfer coefficient based upon the fuel thermal conductivity and molten-fuel particle size in which

$$
\mathrm{h}=\frac{\mathrm{k}_{\mathrm{f}}}{\mathrm{r}_{\mathrm{f}}},
$$

and

$$
A=4-\mathrm{r}_{\mathrm{f}}^{2} \quad \text {, }
$$

where $k_{f}$ is the fuel thermal conductivity. In this study, the units are expressed in cgs unils. The main hindrance to heat transfer is assumed to be due to the thermal conductivity of the fuel. Heat transferred to the sodium is distributed to all sodium in a fuel-coolant-interaction zone so that no thermal gradients exist in the sodium and it can be considered to be in thermodynamic equilibrium throughout the process. The heat of fusion of the fuel is neglected as a first approximation. Due to the nature of the lumpedparameter approach, the model roughly accounts for a finite, though unspecified, fragmentation rate. For more details, the reader should refer to Refs. [25-27].

\section{The Equation of State of the Media}

As described before the solution of the conservation equations requires an equation of state to describe the behavior of the reactor materials, particularly 
in the reactor core and the coolant. The forms of equation of state which have been used are:

a. Murnaghan for fluids:

$$
\bar{p}=p^{n}+c^{n}\left(\rho^{n+1}-\rho^{n}\right),
$$

in which

$$
c^{n}=a_{0}^{2}\left(1+\frac{p^{n} n}{p_{0} a_{0}^{2}}\right) \frac{\rho_{0}}{\rho^{n}},
$$

is closely related to the speed of sound; $a_{o}, P_{o}$, and $\rho_{0}$ are the speed of sound, pressure, and the density at the standard conditions, respectively; i is the isentropic exponent.

b. Isentropic equation of state for the core-gas bubble and the trapped air:

$$
\mathrm{p}=\mathrm{P}_{\mathrm{o}}\left(\frac{\overline{\mathrm{V}}_{\mathrm{O}}}{\overline{\mathrm{V}}}\right)^{\gamma} \text {, }
$$

where $p_{0}$ and $\bar{V}_{0}$ are the initial pressure and volume, respectively; $\gamma$ is the isentropic exponent.

c. Pressure as a function of volume ratio $\bar{V} / \bar{V}_{0}$ for core-gas bubble and air:

$$
p=f\left(\frac{\overline{\mathrm{V}}}{\overline{\mathrm{V}}}\right) \text {. }
$$

d. Pressure as a function of time:

$$
p=f(t) \quad \text {. }
$$

e. Murnaghan for elastic-plastic solids and small distorted fluids:

$$
P=\frac{B_{0}}{B_{0}}\left[\left(\frac{\bar{v}}{\bar{v}}\right)^{B_{0}^{\prime}}-1\right],
$$

in which $B_{0}$ and $B_{o}^{\prime}$ are the bulk modulus and its pressure derivative, respectively. 
Noted that equations related to fuel-coolant interactions described in the previous section are not included here.

\section{J. Differencing Schemes and Convective Fluxes}

There are various ways that the convective fluxes of the conservation equations can be written. The precise form although is irrelevanted to the conceptual formulation used in the ICECO code but is crucial to the accuracy and numerical stability of the calculation. In the ICECO-H code [11] ZIP differencing scheme was used. Recently, capabilities of treating above-core hydrodynamics and sodium spillage have been added to the computer code. In these analysis, fluid motions in the vicinity of the upper internal structure and the reactor cover are sensitive to the directions of the flow. Therefore, a complete donor-cell differencing scheme was incorporated into the code as an option to the ZIP differencing scheme. Also a partial donor-cell differencing scheme has been added to the code to deal with the ongoing effort on the multi-material interactions. Thus, at the present time three types of flux expressions, i.e. ZIP, complete donor, and partial donor were already considered in the mathematical formulation.

According to the description of the ICE technique each of these three flux expressions has advantages and disadvantages. Here, we present various expressions for the convective fluxes $\left(\rho u^{2}\right)_{i, j}$ and ( $\left.\rho u v\right)_{i+\frac{1}{2}, j-\frac{1}{2}}$ of the radial momentum equation and discuss the advantages and disadvantages of each expressions. Since in these flux terms certain variable is not defined at which the flux is to be evaluated some type of interpolation is needed.

The three flux expressions to be considered are described below.

ZIP :

$$
\left(\rho u^{2}\right)_{i, j}=\rho_{i, j} u_{i-\frac{1}{2}, j} u_{i+\frac{1}{2}, j}
$$


Complete Donor:

$$
\left(\rho u^{2}\right)_{i, j}=\left\{\begin{array}{l}
\rho_{i, j} u_{i-\frac{1}{2}, j}^{2} \text { if }\left(u_{i-\frac{1}{2}, j}+u_{i+\frac{1}{2}, j}\right)>0 \\
\rho_{i, j} u_{i+\frac{1}{2}, j}^{2} \text { if }\left(u_{i-\frac{1}{2}, j}+u_{i+\frac{1}{2}, j}\right)<0
\end{array} .\right.
$$

Partial Donor:

$$
\left(\rho u^{2}\right)_{i, j}=\left\{\begin{array}{l}
\rho_{i, j} u_{i-\frac{1}{2}, j}\left(u_{i-\frac{1}{2}, j}+u_{i+\frac{1}{2}, j}\right) \text { if }\left(u_{i-\frac{1}{2}, j}+u_{i+\frac{1}{2}, j}\right)>0 \\
\rho_{i, j} u_{i+\frac{1}{2}, j}\left(u_{i-\frac{1}{2}, j}+u_{i+\frac{1}{2}, j}\right) \text { if }\left(u_{i-\frac{1}{2}, j}+u_{i+\frac{1}{2}, j}\right)<0 \quad .
\end{array}\right.
$$

Also, for both complete and partial-donor differencing schemes:

$$
\begin{aligned}
& (\rho \cup)_{i+\frac{1}{2}, j-\frac{1}{2}}= \\
& \left\{\begin{array}{l}
\frac{1 / 4}{4}\left(v_{i, j-\frac{1}{2}}+v_{i+1, j-\frac{1}{2}}\right)\left(\rho_{i, j-1}+\rho_{i+1, j-1}\right)_{i+\frac{1}{2}, j-1} \text { if } \frac{\left(v_{i, j-\frac{1}{2}}+v_{i+1, j-\frac{1}{2}}\right)}{2}>0 \\
\frac{1}{4}\left(v_{i, j-\frac{1}{2}}+v_{i+1, j-\frac{1}{2}}\right)\left(\rho_{i, j}+\rho_{i+1, j}\right) u_{i+\frac{1}{2}, j} \text { if } \frac{\left(v_{i, j-\frac{1}{2}}+v_{i+1, j-\frac{1}{2}}\right)}{2}<0
\end{array} .\right.
\end{aligned}
$$

The ZIP form is one order more accurate in $\delta \mathrm{r}$ and $\delta \mathrm{z}$ than the donor-cell flux. In addition, it has the advantages of eliminating a nonlinear contribution to instability [28]. For this reason the ZIP flux is of ten preferred for the momentum convection, except in problems the flow direction may play important role in the numerical solutions.

In the donor-cell expressions, either the complete- or partial-donor flux, the finite difference expression is not space-centered, and hence both have low order (in $\delta r$ and $\delta z$ ) truncation errors. This contribute a positive diffusive effect and accordingly tend to automatically stabilize the numerical calculations. The magnitude can be excessive, thus leads to erroneous interpretations. 
Our recomnendation is to use ZIP differencing scheme in the majority calculations, such as the wave propagation, slug impact, expanding gas bubble, fluidstructure interaction, liquid cavitation, and the containment response. The only circumstances in which a complete-donor cell differencing scheme appears desirable is in the analysis of sodium spillage where violent fluid motion near the reactor cover may occur. In fact, except for problems dealing with the sodium spillage, all other problems presented in this report are all using the ZIP differencing scheme.

\section{K. Lagrangian Formulation for Continua and Structures}

Because of history dependence of the constitutive equations Lagrangian coordinate system is employed in the dynamic analysis of structure and solid continua. In view of complicated structure components involved in a typical reactor configuration, several separate programs are employed for analyzing the structural response. Several years ago, a finite-difference thin-shell program has been used in analyzing the response of the primary vessel. This program is now available as an option to the finite-element program in another version of tCECO code [13].

Presently, in the analysis of plug-jump and sodium-spillage problems, the motion of the reactor head, which is assumed tu nave only the rigid body motion under the constraint of the hold-down bolts, is calculated by a finite-difference program.

The shell structure in the reactor containment is now analyzed by a finiteelement scheme developed by Belytschko and Hsieh [29-31]; whereas the elasticplastic solid is analyzed by the finite-difference method utilized by wilkins [7]. To facilitate the numerical treatment of boundary conditions and the arbitrary combination of the solid, the fluid, and the shell structure the equation of motion used in the finite-difference calculation for the solid media are formulated through the use of intermediate nodal forces. Thus, the equation of motion for solid and shells can be written as

$$
M \underline{u}=F^{e x t}-F^{i n t} \text {, }
$$

where $M$ is the mass matrix; $\underline{u}$ the displacements; $F^{\text {ext }}$ the discretized external 
forces; and $\mathrm{F}^{\text {int }}$ the discretized internal forces; dots over the quantity denote time derivative. The equation of motion are integrated explicitly using a central difference scheme. Thus, velocities and displacements in the global coordinates are determined by the following relations

$$
\begin{aligned}
& \underline{\dot{u}}(t+\Delta t)=\underline{\dot{u}}(t)+\frac{1}{2} \Delta t[\underline{u}(t)+\underline{\ddot{u}}(t+\Delta t)], \\
& \underline{u}(t+\Delta t)=\underline{u}(t)+\Delta t \underline{\dot{u}}(t)+\frac{1}{2} \Delta t^{2} \underline{i}(t) .
\end{aligned}
$$

The oftenly used two elements, i.e. quadrilateral torodial continuun solid and conical shell elements, are briefly described. Other two elements, triangular and quadrilateral fluid elements, are given in Ref. [30].

\section{Quadrilatera]. Continuum}

The quadrilateral element can be used to model the elastic-plastic solids in the primary containment such as the radial shield and lead. It also can be used to simulate the compressible fluids with small distortion. In the analysis, the mass of each element is assumed to be equally distributed to its four vertice nodes. Thus the mass associated with each node point shown in Fig. 13 is

$$
M_{0}=\frac{1}{4} \sum_{i=1}^{4} M^{(i)} .
$$

\section{a. Noda1 Force}

The external forces are the pressure forces transmitted from the fluids. These forces are supplied by the fluid-dynamic calculation. The internal forces can be computed from the finite-difference representations similar to the method utilized by Wilkins [7?. In the Lagrangian formulation the equations of motion in the $r$ and $z$ directions are:

$$
\begin{aligned}
& \psi=\frac{1}{\rho}\left[\frac{\partial \sigma_{r r}}{\partial r}+\frac{\partial \sigma_{r z}}{\partial z}+\frac{\sigma_{r r}-\sigma_{\theta \theta}}{r}\right], \\
& \ddot{z}=\frac{1}{\rho}\left[\frac{\partial \sigma_{r z}}{\partial r}+\frac{\partial \sigma_{z z}}{\partial z}+\frac{\sigma_{r z}}{r}\right],
\end{aligned}
$$


in which

$$
\begin{aligned}
& \sigma_{r r}=\bar{\sigma}_{r r}-(p+q), \\
& \sigma_{z z}=\bar{\sigma}_{z z}-(p+q), \\
& \sigma_{\theta \theta}=\bar{\sigma}_{\theta \theta}-(p+q),
\end{aligned}
$$

are the total stress components in the radial, axial, and circumferential directions, respectively; $\bar{\sigma}_{r r}, \bar{\sigma}_{z z}$, and $\bar{\sigma}_{\theta \theta}$ are the corresponding deviatoric stress components; $\sigma_{\mathrm{rz}}$ is the shearing stress; $\mathrm{p}$ is the pressure; $\mathrm{q}$ is the viscous pressure; $\rho$ is the density; $r$ and $z$ are the nodal coordinates in the $r$ and $z$ directions, respectively.

In the finite-difference representation the equations of motion for a generic element shown in Fig. 13 are

$$
\begin{aligned}
\ddot{r} & =-\frac{1}{2 \Phi}\left[\sigma_{\mathrm{rr}}^{(1)} z_{\mathrm{ab}}+\sigma_{\mathrm{rr}}^{(2)} z_{\mathrm{bc}}+\sigma_{\mathrm{rr}}^{(3)} z_{\mathrm{cd}}+\sigma_{\mathrm{rr}}^{(4)} z_{\mathrm{da}}-\sigma_{\mathrm{rz}}^{(1)} \mathrm{r}_{\mathrm{ab}}-\sigma_{\mathrm{rz}}^{(2)} \mathrm{r}_{\mathrm{bc}}\right. \\
& \left.-\sigma_{\mathrm{rz}}^{(3)} \mathrm{r}_{\mathrm{cd}}-\sigma_{\mathrm{rz}}^{(4)} \mathrm{r}_{\mathrm{da}}\right]+\frac{1}{4} \sum_{i=1}^{4}\left[\left(\sigma_{\mathrm{rr}}-\sigma_{\theta \theta}\right) \frac{\mathrm{A}^{\mathrm{n}}}{\mathrm{M}}\right]^{(i)},
\end{aligned}
$$

and

$$
\begin{aligned}
\ddot{z} & =\frac{1}{2 \Phi}\left[\sigma_{z z}^{(1)} r_{a b}+\sigma_{z z}^{(2)} r_{b c}+\sigma_{z z}^{(3)} r_{c d}+\sigma_{r r}^{(4)} r_{d a}-\sigma_{r z}^{(1)} z_{a b}-\sigma_{r z}^{(2)} z_{b c}\right. \\
& \left.-\sigma_{r z}^{(3)} z_{c d}-\sigma_{r z}^{(4)} z_{d a}\right]+\frac{1}{4} \sum_{i=1}^{4}\left[\sigma_{r z} \frac{A^{n}}{M}\right]^{(i)},
\end{aligned}
$$

in which

$$
\begin{aligned}
& \Phi=\frac{1}{4} \sum_{i=1}^{4}\left(A^{n} \rho^{n}\right)^{(i)}, \\
& z_{x y}=z_{x}-z_{y} \text { and } r_{x y}=r_{x}-r_{y} .
\end{aligned}
$$

In the Eqs. (76-78) $\mathrm{A}^{\mathrm{n}}$ is the area of the quadrilateral at previous cycle (n); $M^{(i)}$ is the original mass associated with ith element; also, $\Phi$ must be modified at the boundary of the Lagrangian domain [31]. 
Note from Eqs. $(76,77)$ that the nodal accelerations are functions of the stresses in its four neighboring zones. This formula is quite restrictive in applying to the general problems. Particularly at the boundary of the structure where certain nodes may be connected by one or two quadrilaterals. Thus, to facilitate the treatment of boundary conditions and the arbitrary combination of the solid and thin shell elements the equation of motion are written through the use of the intermediate nodes forces of each element. Thus, for a typical element shown in Fig. 14, the internal nodal forces in the $r$ and $z$ directions are:

$$
\begin{aligned}
& \left(F_{1}\right)_{r}^{\text {int }}=\frac{1}{2}\left[\sigma_{r r}\left(z_{2}-z_{4}\right)-\sigma_{r z}\left(r_{2}-r_{4}\right)\right] \bar{r}-x, \\
& \left(F_{2}\right)_{r}^{\text {int }}=\frac{1}{2}\left[\sigma_{r r}\left(z_{3}-z_{1}\right)-\sigma_{r z}\left(r_{3}-r_{1}\right)\right] \bar{r}-x, \\
& \left(F_{3}\right)_{r}^{i n t}=\frac{1}{2}\left[\sigma_{r r}\left(z_{4}-z_{2}\right)-\sigma_{r z}\left(r_{4}-r_{2}\right)\right] \bar{r}-x, \\
& \left(F_{4}\right)_{r}^{\text {int }}=\frac{1}{2}\left[\sigma_{r r}\left(z_{1}-z_{3}\right)-\sigma_{r z}\left(r_{1}-r_{3}\right)\right] \bar{r}-x,
\end{aligned}
$$

and

$$
\begin{aligned}
& \left(F_{1}\right)_{z}^{\text {int }}=-\frac{1}{2}\left[\sigma_{z z}\left(r_{2}-r_{4}\right)-\sigma_{r z}\left(z_{2}-z_{4}\right)\right] \bar{r}-Y, \\
& \left(F_{2}\right)_{z}^{\text {int }}=-\frac{1}{2}\left[\sigma_{z z}\left(r_{3}-r_{1}\right)-\sigma_{r z}\left(z_{3}-z_{1}\right)\right] \bar{r}-Y, \\
& \left(F_{3}\right)_{z}^{\text {int }}=-\frac{1}{2}\left[\sigma_{z z}\left(r_{4}-r_{2}\right)-\sigma_{r z}\left(z_{4}-z_{2}\right)\right] \bar{r}-Y, \\
& \left(F_{4}\right)_{z}^{\text {int }}=-\frac{1}{2}\left[\sigma_{z z}\left(r_{1}-r_{3}\right)-\sigma_{r z}\left(z_{1}-z_{3}\right)\right] \bar{r}-Y,
\end{aligned}
$$

where

$$
\begin{aligned}
& \bar{r}=\frac{M_{O}}{\rho^{n} A^{n}}, \\
& X=\frac{1}{4}\left(\sigma_{r r}-\sigma_{\theta \theta}\right) \frac{\Phi}{\rho} \text { and } Y=\frac{1}{4} \sigma_{r z} \frac{\Phi}{\rho} .
\end{aligned}
$$

In Eqs. $(79,80)$ subscripts $r$ and $z$ are used to denote the internal nodal forces in the radial and axial directions, respectively. 


\section{b. Stress and Yield Condition}

The calculations of strain rates (or the velocity strains) are implemented by performing line integration along the counter 1234 of a typical Lagrangian element shown in Fig. 14. Evaluating at time $n+\frac{1}{2}$, they have the following form

$$
\begin{aligned}
& \dot{e}_{r r}=\frac{1}{2 A}\left[\left(\dot{r}_{2}-\dot{r}_{4}\right)\left(z_{3}-z_{1}\right)-\left(\dot{r}_{3}-\dot{r}_{1}\right)\left(z_{2}-z_{4}\right)\right], \\
& \dot{e}_{z z}=-\frac{1}{2}-\left[\left(\dot{z}_{2}-\dot{z}_{4}\right)\left(r_{3}-r_{1}\right)-\left(\dot{z}_{3}-\dot{z}_{1}\right)\left(r_{2}-r_{4}\right)\right], \\
& \dot{e}_{\theta 0}=\frac{\dot{v}}{V}-\left(\dot{e}_{r r}+\dot{e}_{z z}\right),
\end{aligned}
$$

and

$$
\begin{aligned}
\dot{e}_{r z} & =\frac{1}{2 A}\left\{\left[\left(\dot{z}_{2}-\dot{z}_{4}\right)\left(z_{3}-z_{1}\right)-\left(z_{2}-z_{4}\right)\left(\dot{z}_{3}-\dot{z}_{1}\right)\right.\right. \\
& \left.-\left[\left(\dot{r}_{2}-\dot{r}_{4}\right)\left(r_{3}-r_{1}\right)-\left(r_{2}-r_{4}\right)\left(\dot{r}_{3}-\dot{r}_{1}\right)\right]\right\},
\end{aligned}
$$

in which the dot over the quantity representing the time derivative of that quantity.

The incremental strains, $\Delta \mathrm{e}_{\mathrm{rr}}, \Delta \mathrm{e}_{\mathrm{zz}}, \Delta \mathrm{e}_{\theta \theta}$, and $\Delta \mathrm{e}_{\mathrm{rz}}$ and the incremental volume charge $\Delta V$ at time $\left(n+\frac{1}{2}\right)$ can be simply computed from the respective rate change modifying by the time step [7].

The deviatoric stress components can be immediately calculated from

$$
\begin{aligned}
& \bar{\sigma}_{r r}^{n+1}=\sigma_{r r}^{n}+2 \mu\left[\Delta e_{r r}-1 / 3 \frac{\Delta V}{V}\right]^{n+\frac{1}{2}}+\delta_{r r}^{n}, \\
& \sigma_{z z}^{n+1}=\sigma_{z z}^{n}+2 \mu\left[\Delta e_{z z}-1 / 3 \frac{\Delta V}{V}\right]^{n+\frac{1}{2}}+\delta_{z z}^{n}, \\
& \bar{\sigma}_{\theta \theta}^{n+1}=\sigma_{\theta g}^{n}+2 \mu\left[\Delta e_{\theta \theta}-1 / 3 \frac{\Delta V}{V}\right]^{n+\frac{1}{2}}, \\
& \sigma_{r z}^{n+1}=\sigma_{r z}+\mu\left[\Delta e_{r z}\right]^{n+\frac{1}{2}}+\delta_{r z}^{n},
\end{aligned}
$$

in which $\delta_{r r}^{n}, \delta_{z z}^{n}, \delta_{r z}^{n}$ are stress corrections due to rotation see Refs. [3,7]. These terms are required if a mass element has rotated in the $r-z$ plane by an 
angle during the time interval $\Delta t^{n+\frac{1}{2}}=t^{n+1}-t^{n}$, the stresses must be recalculated so that they will be referred to the $r-z$ coordinates in their new position.

For the yield condition calculation a invarient $\mathrm{J}_{2}$ is given by

$$
2 J_{2}^{n+1}=\left(\begin{array}{c}
-n+1 \\
\sigma_{r r}
\end{array}\right)^{2}+\left(\begin{array}{c}
-n+1 \\
\sigma_{z z}
\end{array}\right)^{2}+\left(\begin{array}{c}
-n+1 \\
\sigma_{\theta \theta}
\end{array}\right)^{2}+2\left(\begin{array}{c}
n+1 \\
r z
\end{array}\right)^{2},
$$

and

$$
2 J_{2}^{n+1}-2 / 3\left(\sigma_{y}^{n}\right)^{2}=k^{n+1}
$$

where $\sigma_{\mathrm{y}}^{\mathrm{n}}$ is the yield stress obtained from the plastic stress-strain relationships. If $\mathrm{K}^{\mathrm{n}+1} \leq 0$ the deviatoric stresses as calculated in Eq. (82) are used to compute the internal nodal forces. However, if $\mathrm{k}^{\mathrm{n}+1}>0$ the computed deviatoric stresses must be multiplied by a factor of $(2 / 3)^{\frac{3}{2}} \sigma_{y}^{n} /\left(2 J_{2}^{n+1}\right)^{\frac{1}{2}}$ to calculate the internal nodal forcus.

\section{Shell Element}

In the ICECO code the reactor vessel, core barrel, and the core-support structure can be treated as thin axisymmetric shells or plates, in which the analysis accounts for the membrane and bending strengths of the shells, elasticplastic material behavior, and large deformations under transient loading conditions.

A conical shell element developed by Belytschko and Hsieh [29] is utilized to model the thin shells or plates. This element is formulated using a convective (or corotational) coordinate system. In the analysis, each shell element is associated with a convective coordinate system that rotates but does not deForm with the element. Strains are assumed to be linearly related to the displacement of the element relative to its convective coordinates. Similarly, the nodal forces are considered to be linearly related to the element stresses within its convective coordinates.

In the mathematical formulation the convected $\hat{x}$ axis is taken to lie along the line joining the nodes. Cubic polynomial shape functions in $\hat{x}$ are used for 
the transverse displacements and a linear shape functions are employed for the axial displacement. Although the rotation within this type of element is not constant, the rotation with respect to the $\hat{x}$ axis joining the two end nodes of the element is small, since the strains are assumed to be small. Hence the rotation of the $\hat{x}$ axis, $\alpha$, should be a good approximation of the rotational component of the element's displacement.

Th.e deformation displacements are then the nodal rotations with respect to the $\hat{x}$ axis shown in Fig. 15

$$
\hat{\phi}_{i}=\phi_{i}-\alpha \quad(\alpha=1,2) \quad \text {. }
$$

The midplane displacement thus can immediately be expressed in terms of the midplane strain $\hat{\varepsilon}_{m}$.

In terms of the convective coordinates $\hat{x}$ and $\hat{y}$ the strain-displacement relations are

$$
\begin{aligned}
& \hat{\varepsilon}_{x}=\hat{\varepsilon}_{m}-\hat{y} \frac{\partial \hat{\phi}(\hat{x})}{\partial \hat{x}} \\
& \hat{\varepsilon}_{\theta}=\frac{1}{r}\left(u_{r}-\hat{y} \cos \beta \frac{\partial u_{y}}{\partial x}\right),
\end{aligned}
$$

where

$$
\hat{\varepsilon}_{m}=\frac{\partial \hat{u}_{x}^{d e f}}{\partial x},
$$

is the midplane strain; $B$ is the angle of inclination; $u_{r}$ and $u_{y}$ are the global displacements in the radial and axial directions, respectively.

The cubic shape function transverse displacement field is

$$
\hat{\phi}(\hat{x})=\frac{\hat{\phi}_{1}}{\ell^{2}}\left(\ell^{2}-4 \ell \hat{x}+3 \hat{x}^{2}\right)+\frac{\hat{\phi}_{2}}{\ell^{2}}\left(3 \hat{x}^{2}-2 \ell \hat{x}\right) .
$$

The stresses in the convective coordinates are then computed from the stressstrain laws. A multilinear segment representation [32] is employed to simulate the material nonlinearities. The equations for the internal node forces are 


$$
\left\{\begin{array}{c}
\mathrm{m}_{1}^{P} \\
\mathrm{~m}_{2}^{\mathrm{P}} \\
\hat{\mathrm{f}}_{2 \mathrm{P}}^{\mathrm{P}}
\end{array}\right\}=-\frac{1}{\ell^{2}} \int_{V}\left[\begin{array}{c}
(6 \hat{\mathrm{x}}-2 \ell) \hat{\mathrm{y}} \\
(6 \hat{\mathrm{x}}-2 \ell) \hat{\mathrm{y}} \\
\ell
\end{array}\right] \sigma_{\mathrm{x}} \mathrm{dV} .
$$

The other nodal forces are found by using self-equilibrium equations, and have

the form

$$
\hat{\mathrm{f}}_{1 \mathrm{y}}^{\mathrm{p}}=-\hat{\mathrm{f}}_{2 \mathrm{y}}^{\mathrm{P}}=\left(\mathrm{m}_{1}^{\mathrm{p}}+\mathrm{m}_{2}^{\mathrm{p}}\right) / \ell,
$$

and

$$
\hat{\mathrm{f}}_{1 \mathrm{x}}^{\mathrm{P}}=-\hat{\mathrm{f}}_{2 \mathrm{x}}^{\mathrm{P}}
$$

The nodal forces are then transformed into the global coordinates. The internal nodal forces due to the circumferential stresses are computed by the standard linear nodal force-stress relation. Detail derivations can be seen in Refs. $[29,30]$.

\section{Stability Consideration}

For the consideration of numerical stability, the time step $\delta t_{h}$ chosen for the hydrodynamic analysis must satisfy the modified "Courant Condition." The restriction is that fluid must not be permitted to flow across more than one computational cell in one time step; that is

$$
\delta t_{h}<\min \left(\frac{\delta r}{|u|_{\max }}, \frac{\delta z}{\left.T v\right|_{\max }}\right) \text {. }
$$

Since in the plug jump (movable head) problem the axial dimension of the quasiEulerian cell varies with the head displacement and is much smaller than the axial dimension of the regular cell, the time step used at the inception of slug impact is further restricted by

$$
\delta t_{h}<\frac{\delta y}{\left|v_{y}\right|_{\max }},
$$

where $\delta_{y}$ is the axial dimension of the quasi-Eulerian cell, and $v_{y}$ is the axial velocity component in the quasi-Eulerian cell. Typically, $\delta t_{h}$ is chosen 
equal to one-furth to one-third of the minimum cell transient time obtained either frum Eq. (89) or (90).

For the coupled fluid-structure analysis, the time step $\delta t_{s}$ employed for the structural dynamic program must satisfy the following requirements

$$
\delta \mathrm{t}_{\mathrm{s}}=\beta \frac{\ell}{\mathrm{c}_{\ell}}
$$

and

$$
\delta t_{s}=\beta \frac{l}{6 c_{b}}
$$

Equation (91) applies to all elements, Eq. (92) only to flexural shell element. Here $\beta$ is a reduction factor usually between 0.5 and 0.8 ; $\ell$ is the distance between two nodes; $c_{\ell}$ and $c_{b}$ are longitudinal and flexural wave velocities, respectively, and are defined in Ref. [30].

Because the hydrodynamic program uses implicit integration scheme while the structural dynamic program utilizes explicit integration procedure, $\delta t_{h}$ is larger than $\delta t_{s}$. Thus, within each time step several structural dynamic calculations must be performed in order to match one hydrodynamic calculation. The number of structural calculations (or known as sub-cycles) $\mathrm{N}$ can be determined by

$$
N=I\left(\delta t_{h} / \delta t_{s}\right)+1,
$$

where I denotes the integer part of the ratio.

In certain circumstances, the viscosity coefficients $\lambda$ and $\mu$ should be used to damp out the pressure oscillation, particularly in a high-energy excursion. Thus, similar to the treatment of the ICECO technique, the coefficients and $\mu$ should be large enough so that

$$
\lambda, \mu \geq 3 / 2 \rho|\bar{v}|^{2} \delta t+\rho_{\max }(\nabla \cdot \bar{v})\left(\delta r^{2}, \delta z^{2}\right)_{\max } .
$$




\section{Solution Procedure}

We have presented the equations governing the advanced-pressure field for the entire computing domain. In summary the govern difference equations for different regions are: (1) Poisson equation [i.e. Eq. (14)] for the cell full of fluids. (2) Modified Poisson equations [Eqs. (24), (32), (40)] for cells inside or near the perforated upper internal structures. (3) Modified Poisson equation [i.e. Eq. (48)] for the quasi-Eulerian irregular cells under the reactor cover. (4) Relaxation equation [Eq. (50)] for cells near the deformable external boundary. (5) Generalized relaxation equation [Eq. (51)] for cells containing boundaries of deformable internals.

In the analysis, Eqs. (14), (24), (32), (40), (48), (50), and (51) are solved together by the regular iterative technique, which sweep through all the regular and irregular cells in the directions of increasing $i$ and $j$. During each iteration sweep, say at iteration cycle $h+1$, the new value of $\left(p_{i, j}^{n+1}\right)^{h+1}$ is obtained from the corresponding governing equation and is used to replace the old value of $\left(p_{i, j}^{n+1}\right)^{h+1}$. The iteration is terminated when the new values do not differ much from the old values. In other words, the convergence is achieved when

$$
\left|\frac{p_{\text {new }}^{n+1}-p_{o 1 d}^{n+1}}{\mid p_{n e w}^{n+1}+p_{o 1 d}^{n+1}}\right| \leq \varepsilon,
$$

has been satisfied for all cells. Here $\varepsilon$ is usually of the order $20 \times 10^{-4}$ or equal to some other suitable small number.

Figure 16 is a flow chart representing the computation procedure. 


\section{APPLICATIONS}

\section{A. Response of Pool-Type LMFBR}

This study deals with the analysis of wave propagations in a pool-type LMFBR, serving as part of the supporting effort for the PCRV adaptation. The empha$s$ is in on the analyses of the expanding core-gas bubble, together with the response of the reactor internals. Because of relative large dimensions of trapped air and the reactor configuration, excessive interface distortion and long-duration calculation are often involved. Under these conditions, the ICECO code is well-suited for such analysis. Furthermore, the code is attractive since it can treat two-dimensional sliding interfaces, flow around corners, large-amplitude free-surface motion, and can provide a stable solution over the entire spectrum of excursions.

The initial configuration used in the analysis is shown in Fig. 17. In the analysis, the vessel boundary and the core-support structure are both considered as rigid the radial shield and the core barrel are treated as deformable. In order to have better resolution at the material interfaces dense particles are placed at the bubble boundary and the free surface. The initial pressure inside the reactor core is about $27 \mathrm{MPa}$.

A sequence of analytical configurations at the specified times is given in Fig. 18. This shows the expansion of the core-gas bubble and the impact of fluid onto the reactor cover. The distortion of the uubbie-coolant interface and the movenent of the coolant around the upper edge of the core barrel are also clearly shown. Figure 19 gives radial displacement history of the upper core barrel. As it can be seen the solution is very stable.

The code is very efficient. The CPU time is only 20 minutes. The solution extends to $t=110 \mathrm{~ms}$ that is long enough to include all the important transients generated during the excursion. 


\section{B. Above-Core Hydrodynamics Induced by Upper Internals}

Sample problems are given to study the mitigating effect of the perforated upper internal structure on the containment response as well as various modeling techniques available.

\section{The Effect of Upper Internal Structure on the Wave Propagation}

Here, we investigate the effect of the UIS on the wave propagation, pressure loadings, and containment response during an HCDA. The initial reactor configuration chosen for this study is shown in Fig. 20. To reduce the number of parameters involved in the analysis we considered the core barrel and coresupport structure, as well as the reactor cover, to be rigid. The reactor vesscl is made of elastoplastic material. The stress-strain relationship describing the vessel properties was approximated by two straight-line segments. Two calculations are performed: one with and the other without the UIS. In the calculation with the UIS, the perforation ratios for the first four cells from the centerline are $0.40,0.30,0.20$, and 0.10 respectively. The initial pressure inside the core gas bubble is $30 \mathrm{MPa}$.

Figure 21 compares the total forces on the reactor cover as a function of time for the two cases studied. As can be seen the UIS has a pronounced mitigating effect on the slug-impact load. For the case with this siructure the peak force is smaller than without this particular structure.

Figure 22 presents the reactor configurations at three different times for the case with the UIS. The configuration shows how the bubble expands and how the vessel deforms during an excursion. In addition, Fig. 22 indicates significant flow blockage near the bottom plate of the UIS.

To assess the effect of the upper internal structure on the core-gas bubble expansion and energy release we have found from the results that in the calculation without this structure the HCDA bubble volume and the axial kinetic energy of the fluid at the time of the slug impact are $4649 \mathrm{~cm}^{3}$ and $49.15 \mathrm{MJ}$, respectively. For the calculation with the upper internal structure the corresponding values reduce to $4438 \mathrm{~cm}^{3}$ and $41.38 \mathrm{MJ}$, respectively. This demonstrates 
that the upper internal structure also has capability of confining the HCDA bubble expansion and reducing the axial energy of the coolant slug.

\section{Optional Modelings for the Upper Internal Structures}

As mentioned before the ICECO code also can model the UIS by rigid obstacles. Thus, two more cases are investigated, using two different modelings. In the first case, the coolant passageways and the UIS are modeled by rigid obstacles with an equivalent perforation ratio of 0.38 . In the second case, the UIS is modeled by the rigid obstacles with zero perforation ratio, which corresponds to the case where the UIS passageways were completely plugged by the upper core materials during a whole cort disassembly accident. Figures 23 and 24 gives, respectively, reactor configurations at three difierent times. They show how the core-gas-bubble expands, and how the above-core coolant moves during the excursion. We have found that in the case with coolant passageways the peak value of the impact force is $12.10 \mathrm{MN}$, which falls in between the peak values of the two calculations given r reviously in Fig. 2l. Whereas the peak value corresponding to the case without coolant passageway is $7.8 \times 10^{2} \mathrm{MN}$. As expected, it is less than all the respective values obtained from the calculations with the coolant passageways.

\section{Sodium Spillage From the Primary Containment}

\section{Sodium Spillage During an Energetic HCDA}

Analysis of sodium spillage was performed for a 1000-MVe loop-type reactor as shown in 'ig. 25a. The reactor configuration is $8.8 \mathrm{~m}$ in diameter, which consists of an $8.25-\mathrm{cm}$ thick flexible vessel wall, a fixed reactor head. Two leak paths are assumed to exist during the slug impact. The first one is located in the reactor cover, representing the penetration opening generated at the periphery of the small and intermediate rotating plugs of the reactor cover. Such penetration, initially closed, is modeled to open when the inpulse reached a prescribed value. The second leak path is located at the vesse1-head junction to simulate the gap created by the ruptured seal. Since the primary emphasis of this study was placed on sodium spillage through the two leak paths, calculation was begun at slug impact and with a unfform gas bubble in this 
simplified reactor configuration. The axial velocity of the sodium slug is $50 \mathrm{~m} / \mathrm{sec}$ and the initial core pressure is $4 \mathrm{MPa}$.

Figure 25b presents the reactor configuration at $50 \mathrm{~ms}$. The displacement of the reactor cover and sodium ejections from the top penetration and side opening can be clearly seen. Figure 26 shows sodium velocities of the top penetration and the side opening. The results are presented to $50 \mathrm{~ms}$, based upon the fact that the velocity at the penetration reaches its minimum value. Figure 27 presents the total coolant masses ejected from both top penetration and the side opening. Because the size of the side opening increases sharply during the excursion, larger amount of ejected sodium was observed at the vesse1head junction. The quantity and the velocity of the sodium ejected from the primary vessel is a primary concern in assessing the integrity of the secondary containment.

2. Integrated Analysis of Containment Response and Sodium Spillage

An integrated analysis of containment response and sodium spillage in a typical LMFBR is presented. The reactor configuration used in this study is shown in Fig. 28. It consists of a reactor core, a primary vessel, a moveable vessel head, and the holddown bolts. The core barrel and the core-support ntructure are assumed to be rigid. Consequently large impact force is expected and the results are conservative. Two penetration holes are located in the vessel head (shown by the dotted lines) to indicate the locations of leak paths. These penetrations are closed initially but will become open after being acted upon by the impulses of $3.5 \times 10^{9}$ and $1.5 \times 10^{9}$ dyne-sec, respectively. The holddown bolts are assumed to respond elastic-plastically.

Figure 29 presents reactor configurations at 18.9 and $22.6 \mathrm{~ms}$. The expansion of the gas bubble, the fluid motion around the corner of the core barrel, as well as the deformation of the upper containment vessel can be clearly seen. Marker-particle configurations also show some interesting phenomena after the slug impact. In Fig. 293 the penetrations no longer can sustain the impulses generated by the slug impact and suddenly become open. Figure 29b shows that the reactor cover moves upward and certain amounts of coolant already spilled 
out from both the penetrations and the side opening at the vessel-cover junction. The computation terminated at about $23 \mathrm{msec}$ at which time the primary containment already reaches the dynamic equilibrium. The sodium spillage phenomenon is of primary importance for the initiation of sodium fires in the secondary containment.

\section{The Effect of Core-Support-Structure Openings on the Containment Response}

During an HCDA, the pressure wave emanating from the reactor core can propagate into the lower reactor plenum through the core-support-structure (CCS) openings and thereby cause a pressure increase in that region. In the conventional Lagrangian analysis of the primary containment response, the sodium flow through the coolant passageways of the core support structure did not take into consideration the difficulties of large material distortions induced by the openings. Although t'u solution gave conservative results for the slug-impact load and the xper vessel deformation, it underestimated the pressure loading at the in'et-nozzle region. In order to understand the influence of the CSS openings on the responses of the containment and components, as well as on the wave motion in the reactor plenum, we present here an analysis which accounts for the coolant flow through the CSS openings, and compare the results concerning the pressure loadings and bottom-vessel deformations for the cases with and without openings in the CSS.

The reactor configuration chosen for the analysis is shown in Fig. 30. It consists of the reactor core, coolant, radial shield, core barrel, core-support structure, and the primary vessel. The core support structure is modeled as a composite structure made of plates and shells as in the actual structure.

In the calculation, we assume that the flow areas associated with each radial structure member is different from one another, and that the perforated ratio for the four Eulerian cells close to the symmetrical axis decrease outward in the radial direction.

Reactor configurations obtained from the calculation with the CSS openings show different flow characteristics near the core-support structure. 
Because of the presence of the openings in the CSS immediately below the core region, the fluid can penetrate and separate at the boundaries. This is evident from Fig. 31, which gives the reactor configurations at three different times. From these configurations, one can see how the fluid flows across the CSS in the upper plenum and how the fluid flows away from the boundary due to fluid jetting in the reactor lower plenum. One can also see that, at other structural members without perforated openings, the fluid slides along the solid boundaries during the excursion.

Figure 32 shows the pressure loadings in the reactor plenum for the cases with and without openings. Figure 33 presents the axial deformation of the bottom vesse1. Obviously, if the openings of the CSS are not considered, the pressure loading in the reactor lower plenum and the axial deformation of reactor bottom are underestimated. The degree of the underestimation is, of course, dependent on the perforation ratio of the core-support structure.

The pressure loadings near the outlet nozzle and the total forces on the reactor cover obtained from these two cases are also investigated. As we anticipate, the results reveal that the peak pressure and the peak force are larger when there are no openings than when openings exist. However, the differences are very small.

\section{E. Heat Transfer - The Effect of Condensation on the Pressure Loading}

In this study, the effect of condensation in the fuel-coolant-interaction (FCI) zone on the pressure loading over the extended period of time in a single-subassembly hexcan is analyzed. The computational model is shown in Fig. 34a, in which the FCI zone represents a hypothetical accident due to failure of seven pins. From symmetry considerations, only the top half of the hexcan is considered. For the calculations of heat transfer in the FCI zones, the quasi-steady-state model was used. The initial sodium temperature was $672^{\circ} \mathrm{K}$; the fuel temperature was $3115^{\circ} \mathrm{K}$ (molten). The volume fraction of sodium and fuel was 0.0962 each. The rest was assumed to be the steel. Two computations are performed, one with condensation and the other without. To be able to compare the slug energy when the sodium reaches the top of the hexcan, a fictitious cover was assumed on the top of the hexcan. 
The pressure historles in the FCI zone, as well as at location 1 for both cases, are shown in Fig. 34b through 34d, where pressure pulses due to the compression wave $A$ from the liquid expansion, the inertia wave $B$ from the sodium momentum and the reflected wave $C$ from the slug lmpact can be seen. The effect of condensation is readily observed after onset of vaporization. It also delays the slug impact time from $7.78 \mathrm{msec}$ to $8.23 \mathrm{msec}$, and reduces the peak impact forces from $141.6 \times 10^{9}$ dynes to $113.7 \times 10^{9}$ dynes. The reduction of the peak vapor pressure in the FCI zone due to condensation is about 12.2 bars. Comparing the pressure historles in Fig. 34c with that in the FCI zone, it is noted that the pressure wave attenuation is very small in the initial liquid-expansion phase, but it becomes significant after sodium vapor appears in the FCI zone. It is also noted from Fig. 34 d that the pressure pulse due to the reflected wave in the case of condensation (shown by the solid line) has been drastically reduced.

Figure 35 gives the marker-particle configurations of the hexcan at three different times. It shows the extended motions of the sodium, together with highly expanded FCI zones. 


\section{CODE VALIDATIONS}

Several examples concerning with comparisons of code predirtions with exact solution of theoretical method or experimental data and other program results are given. Due to space limitations only important results are provided.

\section{A. Shock-Tube Problem}

This example deals with a shock-tube problem as given in the Problem 非 of the APRICOT program [22]. The problem provides a sensitive test of the dispersion and numerical noise introduced at discontinuities, in particular at the shock wave and at the rarefaction. Figure 36 shows the configuration of the shock tube that has a rigid tube wall and two rigid end caps and is divided into two gas regions by a diaphragm. The internal energies and temperatures are the same on both sides; the density and pressure on the left side (designated by subscript $\ell$ ) are twice the values on the right side (designated by subscript $r)$. At time $t=0$, the diaphragm is removed. A shock wave initiated at the interface is propagated toward the right side, while a rarefaction wave moves in the opposite direction.

This problem has been analysed both in one and two-space dimensions, using ZIP, partial donor-cell and complete donor-cell differencing schemes. Results from 2-D analyses indicated that the transverse fluid motion is very weak, and that the 2-D solutions closely resemble the 1-D solution. Also, the difference of solutions obtained by these three different schemes is also very small, hence we present the solution obtained from the complete donor-cell scheme.

Figure 37 presents the pressure profile at $t=500 \mu \mathrm{s}$, using time step of $1 \mu \mathrm{s}$ in the calculation. The theoretical solution is also included by the dotted lines. The computed pressure profile is in excellent agreement with the theoretical solution. Also, the solution is quite stable, even without using any viscosity. Figure 38 gives pressure profiles for various time steps, illustrating the importance of the time step on the numerical solution. As can be seen that if the time step is too large (e.g., $50 \mu \mathrm{s}$ ) not only instability occurs, but also dispersion takes place at the shock and rarefaction wave fronts. 


\section{B. ANL Experiment on Bubble Expansion and Contraction}

To further validate the code prediction on bubble dynamics, the code was used to correlate the bubble motion of an ANL experiment performed for studying the motion of an HCDA bubble. The test apparatus used in the experiment is shown in Fig. 39. The test vessel, containing a 12-in.-high column of water, was initially closed and covered by a thin diaphragm membrane. The diaphragm was broken by the diaphragm break rod which falls on signal under the effect of gravity. This permits the high-pressure air to expand rapidly into the liquid region. The behavior of the expanding bubble was recorded photographically and a pressure gauge is set up at Location A to obtain the pressure history.

Figure 40 presents the bubble configurations and fluid motions at seven different times. These configurations show how the free surface moves and how the bubble expends and contracts. First, the gas bubble expands spherically and reaches its maximum radius at about $22 \mathrm{msec}$. Then a dent is generated at the bubble vertex, and the bubble becomes toroidal in shape. The dent at the bubble vertex is caused by the fluid hydrodynamic pressure, which exceeds the bubble internal pressure and thus causes the reversal of the fluid motion. Because of the further depression of the ient, the gas bubble becomes unstable and collapses shortly after $41.89 \mathrm{msec}$, at which time several fluid particles already have been observed inside the gas-bubble region.

Experimentally recorded bubble motions at seven different instants are exhibited in Fig. 41. These configurations show how the bubble expands and contracts during the course of motion. The maximum bubble also occurs at $22 \mathrm{msec}$. To compare the results, the positions of the bubble wall along the symmetric axis obtained by the analysis are also shown in this figure.

Figure 42 shows the pressure histories at Location A obtained from the experimental record and the analysis. The results agreed well, except that at the early stage of motion the experimental trace had rapid pressure oscillation.

The good agreement of the analytical results with experimental data indicates that the code is a primising tool for investigating bubble motion during an HCDA, and that the bubble shape is, in general, not spherical. 


\section{SRI Test on Wave Propagation in an Elastic-Plastic Pipe}

The code was used to simulate the piping experiment conducted by Stanford Research Institute (SRI) [33]. To validate the fluid-structure-interaction analysis as well as to illustrate the code performance on the fluid transient in the piping component.

In the experiment, the pressure pulse was generated by a pulse gun which was attached to a length of Nickel-200 pipe. The pipe was $3 \mathrm{in.} \mathrm{OD,} 40$ mils thick, and $10 \mathrm{Ft}$. long, and filled with water as shown in Fig. 43. Figure 44a shows the pressure-time history at position 1 in the pulse gun, which was used is the source pressure in the numerical calculation.

Figure 44b-d shows the computed pressure-time histories at pipe locations $1 \mathrm{~A}, 2$, and 3 , along with the mean values of the SRI experimental pulses. The peak magnitudes and the pulse shapes, as well as the loading and unloading times, agreed well with the experimental results. Because of the effect of plastic deformation, both the computed and test results reveal that the pressure peak is significantly attenuated as the pressure wave propagates to the downstream of the pipe. Since the plastic waves travel much more slowly than the elastic waves, the pressure pulse which causes the pipe to yield is noticeably dispersed as it travels to the downstream.

Figure 45 gives the time histuries of the radial deformations of the deformable pipe at six positions at about $4 \mathrm{msec}$. The SRI posttest measurements of the pipe wall are also given. At positions close to the source, the computed radial deformations compare well with the measured deformations. At positions away from the source, the pipe deformations are overestimated, as noted at positions 60.96 and $91.44 \mathrm{~cm}$ away from the source. The overestimation is probably due to the fact that the positions away from the source are still subjected to certain amounts of pressures, and thus their deformations are not completely recovered. Nevertheless, the analytical solution indeed agrees reasonably well with the experimental results. 


\section{SNP-300 Tests on Wave Propagations in Primary Containment}

To validate the two dimensional analyses of fluid transients and fluidstructure interactions, the ICECO code was applied to the German LMFBR demonstration plant SNR-300.

Three test configurations of the SNR-300 reactor models shown in Fig. 46 will be considered in this paper. The first two models differ only by the rigidity of their radial boundary and the description of the source. The third model is essentially the same as that in Fig. 46b, except that two cylindrical vessels are used. In all cases, the basic test configuration consists of a spherical pressure-volume source immersed within a pool of water, which in turn, is held by a cylindrical container with rigid ends. From the free surface of the water to the top of the container, the space is uccupied by air. The cylindrical walls of the containers are pre-compressed by the rigid end plates with holddown bolts. Rubber seals separate the cylindrical walls from the end plates.

The pressure-volume relationships of the source are derived independently and are given in Tables 1 and 2 . The dynamic response of the structural material is also separately established by appropriate dynamic tests.

In the analytical model, a spherical-shaped source is used to represent the explosive charge. The position of the outside cylindrical boundary corresponds to the mean radius of the vessel. Other assumptions pertain to these three tests are: (a) the top and bottom boundaries are assumed to be stationary, (b) the cylindrical vessel boundary at the bottom is constrained axially and is free to move radially, and (c) the top vessei boundary is assumed to be free of any constraints.

\section{The Thick-Vessel Mode1}

In the thick-vessel test shown in Fig. 46a, the cylindrical vessel was assumed to deform elastically. The pressure-volume characteristics of the source are given in Table 1 . 
Table 1

Source Characteristics for the Thick-Vessel Model

\begin{tabular}{cc}
$\begin{array}{c}\text { Pressure, } \\
\text { Bar }\end{array}$ & $\begin{array}{c}\text { Volume Ratio } \\
\left(\mathrm{V} / \mathrm{V}_{\mathrm{o}}\right)\end{array}$ \\
\hline 411.0 & 1.0 \\
330.0 & 1.3 \\
263.0 & 1.72 \\
182.0 & 2.37 \\
152.0 & 3.13 \\
124.0 & 4.11 \\
109.0 & 5.18 \\
85.0 & 6.7 \\
65.5 & 8.93 \\
50.0 & 12.0 \\
44.0 & 13.9 \\
36.0 & 17.0 \\
30.5 & 20.9 \\
27.0 & 24.0 \\
23.5 & 27.8 \\
20.0 & 31.5 \\
19.2 & 34.8 \\
\hline
\end{tabular}


Table 2

Source Characteristics for the Thin-Vessel Model

\begin{tabular}{cc}
$\begin{array}{c}\text { Pressure, } \\
\text { Bar }\end{array}$ & $\begin{array}{c}\text { Volume Ratio } \\
\left(\mathrm{V} / \mathrm{V}_{\mathrm{o}}\right)\end{array}$ \\
\hline 426.8 & 1.0 \\
380.8 & 1.18 \\
278.9 & 1.60 \\
199.3 & 2.12 \\
111.7 & 2.75 \\
71.4 & 3.47 \\
42.0 & 5.0 \\
22.0 & 8.0 \\
12.2 & 12.0 \\
7.0 & 18.0 \\
3.8 & 28.0 \\
1.95 & 45.0 \\
1.1 & 68.5 \\
\hline
\end{tabular}


The experimental results of Test 114, a representative test pertaining to the thick-vessel configuration, have been published [34]. A sampling of those data is reproduced here for comparison with the ICECO and REXCO results. Comparisons of the pressure histories at the bottom of the tank $(r=10 \mathrm{~cm}$ and $z=0$ ) and at the cylindrical wall $(r=25.5 \mathrm{~cm}$ and $z=36 \mathrm{~cm})$ opposite the source are given in Figs. 47 and 48.

Fairly good agreement of experiment and analysis is obtained for the first high-pressure rise in Fig. 47. The experimental pressure appears to be somewhat overestimated at the early part of the pressure pulse and largely underestimated later by the analytical results. The overestimation of the recorded pressure pulse is attributed to the difference of the boundary conditions at the vessel bottom. In the analysis the bottom vessel is assumed free to move radially, whereas in the experiment the bottom vessel is constrained by the rubber seal. This rubber seal, although has little radial resistance, definitely could create higher reflected pressure near the vessel-plate junction and thus cause the overestimation of experimental pressure. This boundary effect has small influence away from the bottom, as can be seen from the pressure histories at the vessel wall opposite the source shown in Fig. 48. The overall rise and fall of the pressure correlate very well between experiment and analysis.

\section{The Thin-Vessel Configuration *}

The assumptions made in the ICECO model are similar to those for the thickelastic-vessel configuration, except that the thin vessel is permitted to deform plastically. The stress-strain curve assumed for the thin vessel is confined to a bilinear representation. The elastic portion with modulus $\mathrm{E}=20000$ $\mathrm{kp} / \mathrm{mm}^{2}$ extends to the stress of $44 \mathrm{kp} / \mathrm{mm}^{2}$; beyond the yield stress, a plastic modulus $E_{p}=40.96 \mathrm{kp} / \mathrm{mm}^{2}$ is assumed. The pressure-volume source characteristics used with the model shown in Fig. 46b are taken from Ref. [34] and given in Table 2.

Note that the mathematical model used for the sodium-spillage analysis is not the one described in Sec. II.D, but is the one inherited from the conventional Eulerian hydrodynamic methods. 
In the original solution of the thin vessel problem (see Ref. 13, p. 43) it was assumed that air occupies the space between the surface of the water and the bottom of the cover, and spillage of the fluid does not take place [13]. This ICECO solution is reproduced in Fig. 49, showing in sequence the configurations: (a) the initial grid of the analytical model, (b) the configuration at about the maximum vessel deformation, opposite the source, (c) the initiation of impact, and (d) the maximum deformed configuration at the top of the vessel. It is clearly observed that during the initial stage of vessel deformation at the bottom and opposite the source, it deforms not only in the radial direction but also axially. The axial deformation of the vessel at the top is of the order of about $25 \mathrm{~mm}$ before the slug impact. Making an allowance for the contraction of the bolts during the initial deformation of the vessel, which would be a small fraction of one centimeter, we can see an initial opening on the top before impact takes place. The effectiveness of the rubber seals in a case like this should be negligible. Therefore, spillage of Eluid for the thin vessel model could be considerable and the displacement at the top of the vessel may be quite different than what is shown in Fig. 49.

The uncertainty of water spillage through the existing gap at the top of the vessel on the deformation of the vessel led to two additional 1 uns of ICECO. One run permitted fluid spillage over the top of the vessel during slug impact. Since the Eulerian grid was stationary and the vessel top moved within the grid, some initial matching of grid size was made so that spillage would be through at approximately a full grid dimension. The axial grid size was chosen as exactly half of that of the previous model. Consequently, the difference of this run was that the axial grid size was doubled, and air plus water was free to flow radially through the top-most grid during and after the slug impact. The progression of slug impact, fluid spillage and vessel deformation at the top is shown in Figs. 50a through 50d. It may be observed that the top of the vessel now deforms less than in the previous solution shown in Fig. 49. In fact, while the top of the vessel without spillage and air above the water surface was about $17.5 \%$, the permanent set with spillage and no air on the top was found to be about $12 \%$.

As another variation dealing with slug impact and consequent vessel deformation at the top, an ICECO run was made assuming that no air existed at the top 
of the water surface, but spillage of the fluid was not considered. This variation thus only eliminated the air from the first solution depicted in Fig. 49. The resultant vessel deformation of this run was found to be between those derived from the first two solutions. The final vessel deformations of all three analytical solutions are shown in Fig. 51, together with the experimental results presented in Ref. [34].

\section{The Double-Vesse1 Mode1}

The combination of two codes REXCO and ICECO is utilized in this problem to yield the final analytical deformation of the cylindrical vessels. This is necessary because of basic or current limitations of the individual codes. The complexity revolves around the double-connected regions of water, spillage and even mixing of both fluid domains. The REXCO code, which is based on Lagrangian formulation, is handicapped by large deformations which would be a part of spillage over the top of inner vessel during slug impact. The ICECO code at this point of development does not treat an internal thin vessel. Thus, the analytical modeling of the two-vessel problem is decomposed into two parts: the first part involves the REXCO code and provides the initial solution to the problem. The second part makes use of the ICECO code and continues the solution to the end. The source characteristics for both models are the same as for the thin-vessel model discussed before. A bilinear behavior of the vessel is assumed; an elastic portion with $E=2 \times 10^{4}$ $\mathrm{kp} / \mathrm{mm}^{2}$ extends to a yield stress of $39.2 \mathrm{kp} / \mathrm{mm}^{2}$ after which a plastic modulus of $37.3 \mathrm{kp} / \mathrm{mm}^{2}$ is assumed.

The initial stage of the excursion by means of REXCO is extended to the time when the vessels at the bottom and opposite the source have deformed to their equilibrium. At this time ( $1.4 \mathrm{~ms})$ the REXCO output is converted for input of ICECO. An averaging procedure is applied within the fluid to approximate the initial velocities, densities and pressures for the initial conditions of the ICECO solutions.

The ICECO solution commences with a single fluid domain, a single vessel and simulates slug impact and the resultant fluid spillage. A special assumption made in the ICECO solution is that at the restart the vessel is taken to be 
undeformed. The lower part of this new vessel extending to $0.62 \mathrm{~m}$ is now assumed very thick $(0.5 \mathrm{~m})$. Above this point, the vessel retains its actual thickness. The reasons for specifying a rigid vessel portion at the bottom is because at $1.4 \mathrm{~ms}$, the vessel had deformed permanently and further deformation would not be expected; the thick-vessel portion would confine the deformations to small elastic oscillations which should not be detrimental to the overall solution. The actual thickness of the vessel at the top should enable permanent plastic deformation to take place during slug impact. The junction of the thin-thick portions is placed supposedly sufficiently far from the top so that the effective artificial reinforcement of the thickvessel portion should be small. The final vessel deformations are thus derived from the combined REXCO and ICECO solutions are shown in Fig. 52 also containing the experimental data for Ref. [34]. The single vessel ICECO model thus provides the top deformation of the inner vessel.

\section{E. UKAEA-JRC COVA Experiment of High-Energy Excursion Involving Shock Waves and Cavitations}

This example problem deals with ICECO predictions on high-energy excursion to validate the mathematical treatment of shock-wave propagation and liquid cavitation. Although high-energy excursion has an extremely low probability of occurrance, it has large adverse consequences. The trend toward probabilistic risk analysis has led to the study of such excursion for assuring the integrity of the containment system.

Experimentation concerning high-energy excursions was conducted by the UKAEA and JRC-Ispra as part of the Code Validation (COVA) program [35]. The experiment includes the detonations of high explosive charges within a water-filled overstrong cylindrical vessel, as shown in Fig. 53a. This particular experiment consists of nine identical tests, three performed at AWRE-Foulness, three at AEE-Winfrith, and three at JRC-Ispra. Pressure and impulse measurements were taken at twenty locations on the floor, wall, and roof at three different planes. 
Statistical analysis shows that the recorded data are of high quality. Thus, this COVA experiment has been chosen as a typical problem in the APRICOT program $[22,36]$.

Results [36] of the APRICOT code-comparison exercise revealed that on the floor and the wall most Lagrangian hydrodynamic code provided solution in fair agreement with experimental data. However, the majority of the Lagrangian calculations overpredicted the impulses on the floor, particularly near the center of " the tank. They also produced quite spikey pressure histories on the roof, even some using the sophisticated cavitation model developed by Rayleigh to account for the rate of growth and collapse of cavitated bubbles in water.

Here, numerical calculations were made with the ICECO code, using ZIP differencing scheme. All calculations cover important physical events without utilizing any complementary mechanism, such as artificial viscosities, rezoning, mesh stabilization and regularization, as well as prescribed grid motion. Water cavitation and spalling are treated by the "Pmin" model mentioned in Sec. II.G.

We have found from the results that (1) the ICECO code not only predicts correct1y the pressure pulses on the floor and the side wall, but provides good resolutions of wave profile on the roof; and (2) the material properties of the vessel wall play an important role in calculating the impulse on the floor. Consequently, the discrepancy between the Lagrangian analyses and experimental data can be resolved if the vessel wall is assumed to be deformable.

For completeness, two calculations of the COVA experiments were made with the ICECO code, using vessel material properties as a parameter. One calculation considered the vessel wall to be rigid; the other assumed the vessel wall to be elastic, with the wall thickness taken to be $4 \mathrm{~cm}$.

In the analysis, the physical system is discretized into $25 \times 42$ Eulerian cells as suggested by the APRICOT committee. This mesh configuration is relatively coarse in which the explosive charge was inftially represented by two Eulerian cells. The Inftlal pressure of the explosive charge is $116.04 \mathrm{k}$ bar. Results are brlefly described below. 


\section{Rigid-Wall Solution}

Correlations of the computed pressures and impulses with the experimental results of all gauge positions are made. Wave shapes and arrival times are also compared. In general, the code can predict exceptionally well with the pressure pulses on the floor and wall, including both the incident shock waves, and reflections from the floor and wall and slug impact. However, on the floor the calculated pressure pulses generated from the wall of the tank are higher than those recorded in the experiment due to rigid-wall assumption. Because of space limitation, pressure pulses on the floor and wall are not presented here but are given in Ref. [37].

The computed and measured ilupulses on the floor, wall, and roof are given in Fig. 53b-d. In general the calculated impulses are in good agreement with the experimental results. However, the predicted floor impulses are higher than those measured, especially near the center of the tank. As mentioned before, this is due to the rigid-wall assumption which causes the overestimation of the reflected pulses, and in turn, the final impulse values.

Figure 53e depicts the reactor configuration at $1.0 \mathrm{msec}$, showing also the early stage of the bubble configuration. Because of strong wave reflections from the floor and the wall of the tank several jet-like spikes were observed which penetrate the boundary of the gas bubble. Since the code has an accurate scheme for treating highly contorted coolant surface, the collapsing of the gas bubble at this stage of excursion can be avoided and numerical calculations can be carried out continuously without any interruption.

The phenomenon of roof impact in the COVA experiment is very complex, due to the presence of cavitation and spalling at the coolant free surface. This complex phenomenon, in conjunction with the explicit integration algorithm for the conservation equations, impose certain difficulties in the numerical calculations obtained by the Lagrangian codes. Thus, nearly all the Lagrangian analyses showed considerably spikey pressure histories on the roof even sophisticated cavitation model and large amount of artificial viscosities are used as the smoothing mechanisms. Since the ICECO code uses the implicit integration scheme, the solution is stable and the adverse effect of fluid cavitation on 
the numerical results is less severe than those of the explicit Lagrangian calculations. This can be seen from Fig. 54, which depicts respectively the experimental and computed pressures at roof gauge $\$ 18,10 c a t e d 16.0 \mathrm{~cm}$ away from the axis of the tank (see Fig. 53a). It indicates that the predicted pressure deviates only slightly from the recorded one and correlates well with the experimental data.

\section{Deformable-Wa11 Solution}

From the results of rigid-wall analyses obtained from ICECO and other Lagrangian codes it is shown that although the propagation of the primary pulse to the tank wall and floor was calculated accurately, secondary pulses to the wall, arising from reflections from the wall and the axis of the tank were overestimated in the code predictions. The overestimated secondary pulses further lead to the overprediction of impulses delivered to the floor of the tank, particularly near the centerline.

Recently, we have found that the material properties of the overstrong vessel play a significant role in calculating the strength of the reflected waves, and that even the relatively thick wall used in the covA experiments can temporarily absorb a significant portion of the incident energy and delay releasing this energy for several hundred microseconds. This gives a partial explanation of the unexpectedly low secondary pulses observed in the experiment. Thus, the calculation was rerun with the ICECO code, allowing the vessel wall to deform elastically. The results are given below.

Figure 55 shows pressure and impulse histories at gauge 1 , in which the two ICECO calculations are compared with experimental results. The diagrans on the right-hand side of these figures show the improvements achieved by considering the thick wall to be elastic. Figure 56 shows the impulses at gauge positions 1-7 for the two cases studied, together with the experimental results. The impulses obtained from the elastic-wall analysis agree very well with the recorded data. 


\section{F. SRI Complex Vesse1 Test SM-2 on Structural Response to a HCDA}

Recently, a series of experiments (referred as SM tests) have been performed by SRI International using a $1 / 20$ scale model of the Clinch River Breeder Reactor (CRBR) to study the effects of various structural components on the cover loading and vessel response [38]. These experiments consist of five tests. Test SM-1 was a static test of the reactor cover. The other four tests SM-2 to SM-5 were dynamic tests performed on the scale model of the CRBR. This report describes the ICECO code predictions of the SM-2 test.

The test model of SRI SM-2 is shown in Fig. 57. The reactor vessel was made from $\mathrm{Ni}-200$ to simulate the stress-strain relationship of the Type 304 stainless steel at the reactor operating temperature. The core barrel was a thin aluminum cylinder while the radial shield was made of a stack of segmented steel rings. A Mylar diaphragm is placed above the charge canister to prevent water from entering into the source region. Water was used to simulate the coolant. Eight pressure gauges and ten strain gauges were used in the experiment to record pressure pulses and dynamic strains. Their positions are shown in Fig. 57.

The mathematical model used in the ICECO analysis is shown in Fig. 58a, where the fluid j.s denoted by marker particles. The initial position of the coregas bubble is represented by approximately 18 Eulerian cells located at the lower left corner. The properties of the reactor vessel and core barrel were taken from the test data provided by SRI. The radial shield was treated as an elasto-plastic material with low yield strength. It should be mentioned that the value of the yield stress of the shield material was calculated from the frictional forces between the segmented rings, and that once the pressure force exceeds the frictional force, the segmented rings are allowed to slide and to become separate.

Two types of input data can be used to describe the behavior of the core gas; the pressure-volume $(p-v)$ relationship and the pressure-time $(p-t)$ history of the core gas. Calculations using the $p-v$ as the input may not be accurate enough if errors have occurred in the measurement of core-gas-volume increase during the process of source pressure determination. On the other hand, the 
p-t history of the core gas can be taken directly from the pressure transducer readings of the experiment. Thus, the $\mathrm{p}-\mathrm{t}$ history could provide a more accurate input and is used here. Because of space limitation, only important results are presented in this report.

Figure 58b shows the computer generated reactor configurations after the slug impact. The deformations of the core barrel and reactor vessel as well as the expansions of the core-gas bubble can be clearly seen. Figure 59 shows a comparison of pressure histories of gauge P5. As can be seen, the agreement between the analytical result and the experimental measurement is very good. Figure 60 compares the calculated vessel and core-barrel deformations with the post-test measurement. The agreement is exceptionally good, particularly the lower portion of the reactor vessel. 


\section{THE COMPUTER PROGRAM}

The computer program ICECO-CEL (ICECO-III) is written in FORTRAN IV for the IBM 370/195 computer to analyze the fluid transients, fluid-structure interaction, and the structural response. To assist the user in understanding the program, we first briefly present information concerning the identification of the cell flag, the particle set-up procedure, the slug-impact indication index, modelings of the flexible vessel, deformable internal and movable head cover, as well as the hydrodynamic-structural coupling and etc.

\section{A. Ce11 Identification}

Several types of flags are used in the computer progran to appropriately identify the Eulerian cell, gas-bubble location, the orientation of the boundary segment of the structure, and the free-surface orientation. Here, we briefly describe these flags.

\section{General Ce11 Flag}

First, the cell flag is a numerical number assigned to each Eulerian cell to indicate the cell type. This number will be included in the detailed printout along with other physical variables. From the cell flag as well as the marker-particle configuration generated by the film subroutine, one can visualize the domain of the fluid and the configurations of the gas bubble, the core-gas region, and the boundary of the structures. The cell within a computing region may be one of the following types: (1) A Dummy (D) or a fictitious cell does not contain fluid and falls outside the system boundary. This cell is equivalent to the boundary cell of the MAC method and is used solely for computation purpose $(F=1.0)$. (2) A full (F) cell contains fluid and has no empty cells for neighbors on any of its four faces $(F=2.0)$; thus, this cell denotes part of the fluid region. (3) A surface (S) cell partially contains fluid, but has at least one empty cell among the neighbors on its four edges $(F=3.0)$; this cell defines the free surface or the bubble-coolant interface. (4) An empty (E) cell does not contain fluid ( $F=4.0$ ), and represents the region of gas bubble or the cover gas. (5) An obstacle (OB) cell represents rigid obstacle and does not contain fluid $(F=5.0)$. (6) A boundary ( $B$ ) cell 
contains a segment of the deformable vessel $(F=6.0)$. (7) An arbitrarilyshaped boundary $(A B)$ cell has boundary of a rigid structure with boundary not ccinciding with the Eulerian grid $(F=7.0)$. (8) An internal boundary (IB) cell possesses a boundary segment of the deformable internal structure $(F=8.0)$. (9) A quasi-Eulerian (Q) cell is an irregular cell underneath the reactor cover where sodium spillage and plug jump may occur $(F=9.0)$. (10) A perforated structure (PS) cel] contains either an interior portion or a boundary segment of the perforated structure such as the upper internal $(F=10.0)$. (II) An internal structure (IS) cell contalins interior of a thick solid structure but not tie boundary $(F=11 .(1)$. Fite that there is no fluid (marker particles)

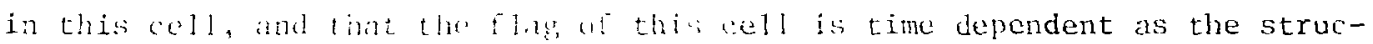

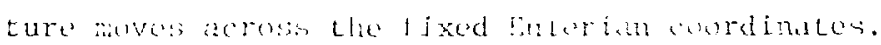

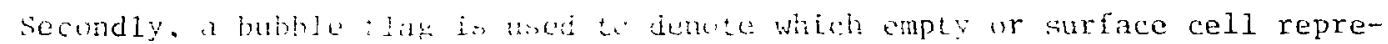

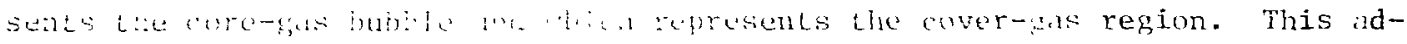

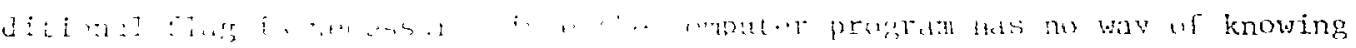

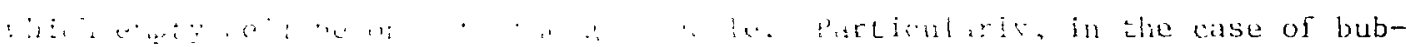

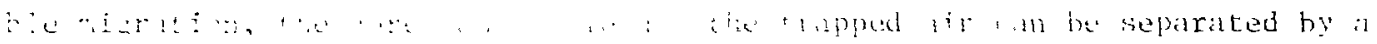

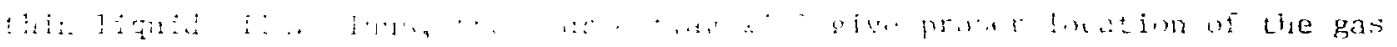

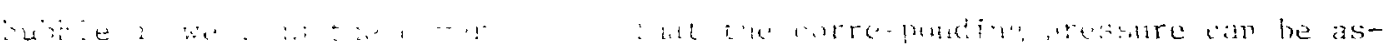

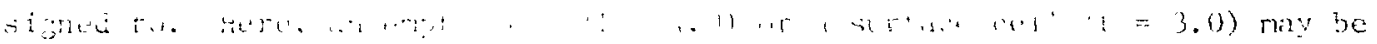

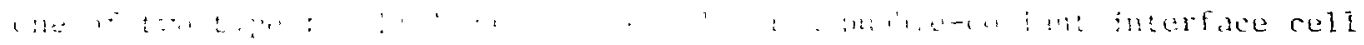

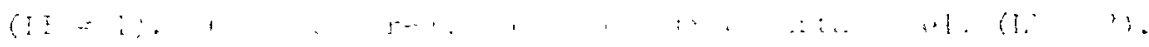

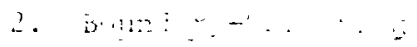

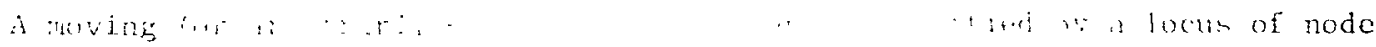

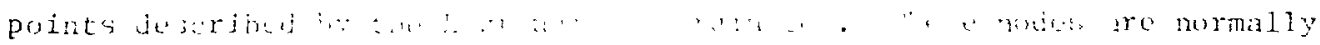
placed a

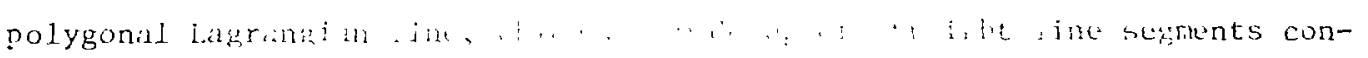

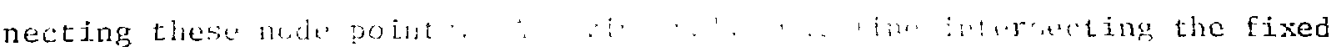

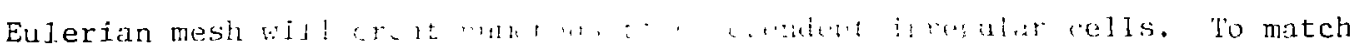

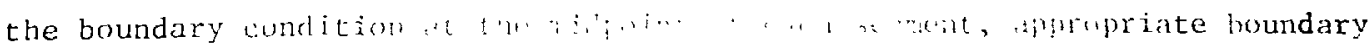
flags must be extihitiniter. 
A boundary flag identifies the Eulerian cell that contains the solid materials and provides geometricai information of how vessel segment is oriented with respect to the Eulerian cell. At present, 15 flags have been considered for all possible intersections of the Lagrangian boundary with the fixed Eulerian grids. These flags are given in Fig. 61 where the boundaries of the structure are indicated by cross-hatch lines. If additional flags are needed, they can easily be incorporated into the computer program.

In calculating fluid-structure interactions involving deformable internals, it is desirable to introduce multivalued variables such as $u, v, p, p$ near the deformable structure as described detailedly in the ICECO-II Report, ANL-78-103 [13]. To accomplish this objective, a criterion (or a flag) must be established to indicate the position of the fluid relative to the structure. Here, we refer to Fig. 61 shown previously and assume that the fluid is at the left when the flag KF equals to $1,9,11,59,69$, or 79 ; the fluid is at top when $K F$ equals to 8,12 , or 13 ; at right when $k F$ equals to 4,6 , $\wedge r 14$; and at bottom when $K F$ equals to 2,3 , or 7 .

\section{Surface-Ce11 Flag}

In the analysis velocity components are defined at the middle points of cell boundaries as shown previously jn Fig. 2. In a surface cell, the position where the velocity is defined may be outside the fluid region. This happens of ten when a surface cell has empty neighbors. Here two flagging schemes are established to identify the number and position(s) of the velucity components located outside the fluid region.

\section{a. Conventional Flagging Sclieme}

The conventional flagging scheme is the one being used in the Eulerian hydrodynamic methods such as MAC [39], SMAC [40], ICE, and their extended technique. The scheme simply searches the positions of the empty cells relative to a surface cell. Figure 62 presents 12 cell flags, indicating possible arrangement of empty cells relative to a surface cell. These flags, representing by different numerals, provide locations (shown by dots) of velocities outside the fluid boundary. 


\section{b. Detailed Flagging Scheme}

Since the conventional flag depends on the positions of empty cells relative to a surface cell rather than on the position and orientation of the free surface, the solution obtained is approximate. The detailed flagging scheme approximately calculates the free-surface orientations and finds which velocity locations are outside the fluid region similar to the manner of determining the boundary flags in the fluid-structure interaction. Presently, 12 different flags (see Fig. 63) are designed for the surface cell with the heavily line indicates the free surface loundary and the arrow points into the fluid region. This flagging scheme has not been generalized enough to take care of all the problems. Thus when apply this scheme we should note that: (1) It can only be applied to the case the bubble is surrounded by the fluids. In other words, the first and last marker particles defined the bubble boundary must be on the symmetrical axis. (2) The marker particles described the bubble boundary and the free surface (if any) must be numbered in such a way that as one advances from i-til particle to $(i+1)$-th particle the fluid is on the left of the interface. (3) The scheme cannot be applied if the distance between two consecutive interfacial particles are greater than one cell.

In general, the numerical solution based on the conventional flagging scheme can give satisfactory results for majority cases. However, in the situation the bibble-coolant interface has large curvature the refined flag might provide a better solution.

\section{B. Other Information}

\section{Surface-Cell Velocity Calculation}

Once the locations of the unknown velocity components are determined by either one of the two flagging schemes, their values are computed similar to those of the MAC method in treating the open sides of the free surface cells. For one unknown velocity case (configurations 1, 2, 4, 8 in Figs, 62 and 63), or two adjacent unknown velocities case (configurations 3, 6, 9, 12), the appropriate $\mathrm{u}$ and/or $\mathrm{v}$ may be calculated precisely from the mass equation. For the case of three unknown velocities (configurations $7,11,13,14$ ) we customarily 
assume the interface is flat and first compute two $u$ or two $v$ based on the inviscid boundary condition; then compute the remaining one unknown using the mass conversation aquation.

\section{Marker-Particle Setup and Slug Impact}

To create the fluid inside the computing regiun, the particle-setup cards are needed. The computing program requires one card for each set of marker particles. The shape of the particle set is limited to being rectangular, as illustrated in Fig. 64.

For each set of marker particles, the number of particles to be created in the $r$ and $z$ directions should be specified through parameters NX and NY. Four dimensions - XO, YO, DXK, and DYK - are also needed to describe the geometry of a given particle set. The FORTRAN variables XO and Yo represent the coordinates of the first particle; DXK and DYK denote the space increments in the $r$ (or $x$ ) and $z$ (or $y$ ) directions, respectively.

Both the interior and interfacial particles are denoted by the same FORTRAN variable. To distinguish these particles, the particle sets defining the coregas bubble should be read in first and the particles representing the interface between the coolant and the cover gas should be read in next. After the input of interfacial particles, the interior particles can be input in any order. The particles used to define bubble boundary and the free surface must be set up or numbered in such a way that as one advances from (i)th to $(i+1)$ th particles the fluid region is at the left-hand side of the interface boundary.

At the beginning of slug impact, the marker particles originally positioned on the free surface move upward and impact the reactor cover. In the numerical approach, the Eulerian cell underneath the cover head will be changed from a surface cell to a full cell and allowed to produce a pressure on the cover. Since the free surface generally exhibits a curvature, and slug impact occurring in the cell underneath the cover takes place at a different instant, a criterion must be established so that the programs will know when to change the cell flag and shift the pressure calculation. This criterion can be specified through the parameter KIMP, which means the minimum number of particles for 
each cell required to contact the vessel head to imply the slug impact. In a two-dimensional excursion, this parameter can be set equal to one-half of the number of particles located at the free surface in each surface cell. In the one-dimensional case, the slug impact begins when all free-surface particles have been pushed upward and have contacted the cover. Nevertheless, the specification of this parameter depends on the physical judgment of the user.

\section{The Computing Domain and Modeling Technique}

The computing mesh used in the ICECO analysis is similar to the MAC method in which a belt of dumm (fictitious) cell outside the boundaries of the real computing mesh are used for the computational purpose. The mesh boundaries are assumed to be the rigid walls so that the normal velocities are set equal to zero at all the calculation cycles in order to conserve momentum exactly. This setup is intended for the hydrodynamic problems with boundaries coincide with the mesh boundaries. Thus, one can easily figure out the mininum cell numbers for a given domain by adding two cells in both the radial and axial directions.

However, for problems involving flexible external boundaries shown in Fig. 65, attention should be given regarding how to determine the minimum cell numbers in order to conserve the storage. The guideline is that one should keep at least one column (or one row in the case of movable head and curved reactor bottom) of empty cells between the dummy cells and the cell containing the rightmost (or uppermost) external boundary segments. The reason of the arrangement is that the velocities outside the boundaries (shown by arrows) are needed in matching the boundary conditions at the fluid-structure interfaces, and that these velocities generally have nonzero values which are different from the zero velocity set at the mesh boundaries. With this background in mind, one can compute a priori the required cell numbers for problems involving small or moderate displacement of external boundaries. In the case of large displacement, one may either leave more than two empty cells outside the boundaries or perform a few try runs to find out the cell numbers containing the farest boundary segment and decide the required cell numbers from there. 
Another thing noteworthy to mention is the initial positions of the flexible external boundaries. Again, referring to Fig. 65 our guideline is to place the external boundaries (such as vessel, bottom of the cover if movable, and reactor bottom, etc.) slightly off the grid lines at where velocity components (shown by dots) are rigorously computed from the momentum equations. Thus, in the fluid-structure-interaction analysis the velocity vector of the virtual particle located at the midpoints of the boundary segment will depend less significantly on the velocities outside the boundaries. Although we cannot avoid this situation entirely since the boundary could move in an arbitrary manner, at least we treat the interaction precisely at the beginning of the deforming stage.

Finally, for problem involving deformable internals we should also keep in mind that the present computer program can only handle small displacement of reactor internals. Also, the program has the restriction that the internal boundary cell contains only one midpoint of the boundary segment, as shown in Fig. 66. Therefore, in she numerical modeling we should lump the radial shield and the core barrel together and skillfully avoid the situation where one Eulerian cell has tiwo interfacial segments.

\section{Hydrodynamic-Structure Connectivity}

In the coupled fluid-structure problem involving interactions of coolant with primary vessel and various deformable internals it is necessary to transmit certain information back and forth between the hydrodynamic and structural calculations. These two calculations are combined as follows: (1) the hydrodynamic program first computes pressure on the load line representing the fluid-structure (or Eulerian-Lagrangian) interface, (2) the pressure is treated as input external (or driving) force to the structural program, (3) the structural program returns the displacements, velocities, and accelerations at the fluid-structure interface to the hydrodynamic program, and (4) the hydrodynamic program performed iteration on the advanced-time pressures using the returned values and computes a new improved pressure at the boundary. The procedure of (1) to (4) is repeated until a prescribed terminating cycle (or time) is reached. 
To provide proper communications between the hydrodynamic and structural calculations we have established two numbering systems for hydrodynamic and structural computations, respectively. The first one is point/segment system for hydrodynamic calculations. The second one is the node/element system used for finite-element discretization of the structure.

In the hydrodynamic calculation using point/segment system the fluid is assumed to be on the left-hand side of the load line that connects the beginning and the terminating points. On each load line the 'point' and hence the 'segment' must be numbered in a consecutive manner. Thus, two point numbers are given at the intersection of two load lines, as shown in Fig. 67a. In this figure ordinary numbers indicate 'point' numbers, numbers with prime (showing only the first and the last one each load line) are the 'segment' numbers.

In the structural calculation conventional node/element numbering system is used. The 'node' number on the pressure load line is restricted by the numbering rules of the finite-element discretization, in which only one nodal number (excluding the dummy nodes for the shell element) can be given at the intersection. Figure $67 \mathrm{~b}$ shows the numbering system for the finite-element structural dynamic calculation. Here, the circled numbers represent 'node' numbers; the starred numbers indicate 'element' number. Understandably, numbers indicating 'dumy' nodes as required by the finite-element thin shell calculations are excluded for the purpose of simplification.

The format and algorithm for reading-in the segments/points numbering are the same as for reading-in the nodes numbering. Hence, automatic generation of the numbering can be used when the numbering is increased by a constant. The format and algorithm for reading the segment/points connectivity information are the same as for reading-in the finite element element/nodes relationship. Again, automatic generation of these data can be utilized if all the numbers are Increased by one. 


\section{Computer Output}

\section{Input Verification Printout}

The code is user-oriented. It is constructed in such a way that the user can easily understand and verify his input cards. Thus, all the information generated by the input cards at the beginning of cycle are printed with headings. These printouts are briefly described below.

\section{a. Data for Hydrodynamics}

Complete input cards for hydrodynamics are printed in accordance with the input instruction, including the card number and variables FORTRAN names. Such output provides important information concerning: (1) title of the run; (2) the size of the computation region; (3) specification of plot to be done by the CALCOMP plotter, index for problem continuation; (4) important indexes or parameters indicating whether or not the problem involves slug impact, fluidstructure interaction, moving cover and sodium spillage, deformable internals, flow through perforated structure, and etc.; (5) characteristics of the source pressure; (6) coordinate-system options, rigid-obstacle uptions, rigid thin shell (or plate) options, ZIP or donor-cell differencing options, and etc.; (7) marker-particle configuration; (8) information for deformable internal structure, moving cover and sodium spillage, flow through perforated structures.

b. Data for Structural Dynamics

(1) Number of nodes, elements, materials, nodes at which special displacement conditions are specified, structura]. Lime increment, and the number of load lines; (2) properties of material used; (3) a table of three columns showing the radial ( $x$ ) and the axial (y) coordinates of all nodal points; (4) a table of eleven columns showing the element number, its associated four nodes (N1-N4), three blank numbers for future use, problem-characteristic number (PTYP), material type number (MTYP), and the element type number (ND); (5) a table of four columns showing the node number at which special displacement constraints are set and describing the constraints. One in the $x$ column indicates that 
motion in the radial direction is not permitted. One in the y column indicates that motion in the axial direction is not permitted. The angle column shows the angle between a direction along which motion of the node is not permitted and the global x coordinates; (6) hydrodynamic-structure connectivity, point/ segment and node/element relations; (7) table of $Q$ array and the required array storage.

\section{General Printout}

\section{a. A11 Problems}

At every NDTGEN cycle the program prints: (1) under title ' $K T$ CK' the number of marker particles per each Eulerian cell that have contacted with the cover; sush printout appears if slug impact is expected and no quasi-Eulerian (irregular) cells are involved; (2) the radial, axial, and total fluid-kinetic energy, total energy in the system, total fluid internal energy, original core energy, and energy balance discrepancy; (3) pressure, energy of the coregas bubble, volume of gas bubble at the previous ( $n^{\text {th; }}$ and advanced-time $\left(n+1^{\text {th }}\right)$ cycles, as well as the criginal core volume; (4) information similar to (3) for the trapped air; (5) pressure in the core-gas bubble, trapped air, and time if pressure-time $(p-t)$ histories are used as source pressures; in this case printout items (2)-(4) mentioned above are omitted; (6) cycle number, time, and time step; (7) solution time in the structural program, allowable time step, number of subcycles, time step of structural analysis; (8) time, number of iterations needed, calculated maximum convergence criterion, and fluid volume.

\section{b. Moving-Cover and Sodium-Spillage Analysis}

If problem involves moving cover and sodium spillage that is analyzed by the control-volume technique, the program prints: (1) at every cycle under title 'penetration $\mathrm{x} x$ is opened', the radial cell number of the penetration, the calculated and the allowable impulse if the penetration becomes open; (2) at every NDTGEN cycle under title 'COVER' the cover axial cell number (JC), cover height, total force on the cover, cover position, cover velocity, cover acceleration, bolt force, pressure in cell $(4, \mathrm{JC})$, pressure in cell $(4, \mathrm{JC}-1)$, 
flag of cell $(4, \mathrm{JC})$, flag of cell $(4, \mathrm{JC}-1)$; (3) for every NDTGEN cycle and every contacting particle under title 'REFLAG CK CONTACT' the particle number, its radial cell number (I), number of particles contacting the cover associated with cell I, particle's radial position, particle's axial position, cover axial position, axial velocity at bottom of the irregular cell, and the cover velocity.

\section{Optiona1 Printout}

Optional printouts are determined by each frequency specified in card type 11 in the input instruction. The contents of these printouts are briefly described below.

\section{a. Detailed Cell-Variable Output}

For every NDTCP cycle the program prints the detailed information for each Eilerian cell, including the radial cell number $I$, the axial cell number $J$, sonic speed $c$, fluid velocity in the coolant passageway $\tilde{v}$, radial velocity $u$, source term of the radial momentum requation $R$, source term of the axial momentum equation, pressure $p$, source term of the Poisson equation $G$ (or $H$ in this report), cell flag F, free surface or boundary flag KF, bubble flag LF, flag $\mathrm{NF}$ for cell around the deformable internals.

\section{b. Particle-Position Output}

For every NDTPP cycle the program prints marker-particle information, including particle number, its radial position, and axial position.

\section{c. Boundary-Flag Output}

For every NDTFLG cycle the program prints boundary flags information of each segment in a six column tabulated form under the title 'BDYFLG', including segment number $M$, its radial cell number 1 , axial cell number $J$, first boundary flag KF1, second boundary flag KF2, radial position of the first point RV(M), axial position of the first point $\mathrm{ZV}(\mathrm{M})$, radial position of the second point RV $(M+1)$, axial position of the second point $\mathrm{ZV}(\mathrm{M} \dot{\mathrm{r}} \mathrm{l})$, segment slope $A$, segment axial distance $B$, and cell flag $F(I J)$. 


\section{d. Deformable-Internal Output}

For every NDTINT cycle the program prints multivalue variables near the reactor internals in fifteen columns, including radial cell number $I$; axial cell number $\mathrm{J}$; fluid densities on the left- and right-hand sides of the internal RHO(LR); fluid densities above and below the internal RHO(TB); radial velocities on the left- and right-hand sides of the structure $U(L R)$; radial velocities above and below the internal $U(T B)$; source term of. the radial momentum equation $R$; axial velocities on the left- and right-harld sides of the structure V(LR); axial velocities above and below the structure $\mathrm{V}(\mathrm{TB})$; source term of the axial momentum equation $S$; pressures on the left- and right-hand sides of the internal $\mathrm{P}(\mathrm{LR})$; pressures above and below internal $\mathrm{P}(\mathrm{TB})$; hydrodynamic pressure $\mathrm{p}$; boundary flag of the first interface $\mathrm{KF} 1$; boundary flag of the second interface KF2; segment number of the first interface IVI; segment number of the second interface IV2.

Note that the format of variable printout may be changed from time to time. However we don't anticipate any difficulty of understanding the meaning of these variables. For instance if radial velocity $U$ is printed in four rows under heading $U$ (LTRB), then these four values indicate the radial velocities corresponding to the fluid at the left, top, right, and bottom side of the structure, respectively. Similar designation applied to other field variables.

\section{Pictorial Display}

\section{a. FILM Output}

The program provides reactor configuration generated by the FIIM at every NDTP cycle. Thus, structural deformation, marker-particle movements, core expansions, and flow patterns can be seen on a series of these displays.

\section{b. Results for CALCOMP Plot}

After the calculation is terminated, the program prints out all the information stored on binary tape 2 to be used later for plotting purposes. The information is printed in the form: (1) problen title; (2) number of cycles 
stored and number of values stored; (3) a table of 10 columns consisting of cycle number, time in seconds, and eight columns of values stored. This information in printed repeatedly until all values are printed. Each column of values is headed by $\mathrm{KXP}(\mathrm{I}), \mathrm{KYP}(\mathrm{I})$, and $\mathrm{KZP}(\mathrm{I})$ identifying the zone and the type of variable stored as supplied in card Type 4.

\section{Program Execution and Limitations}

As will be described later that the dimensions of common blocks inside the computer code is not dynamically allocated. We intend to make the dynamic allocations of the program in the near future so that one can save the core storage for different applications. However, the available load module has a capacity of executing problems with 420 Eulerian cells and 4000 marker particles, which is large enough for most containment analyses. Nevertheless, adjusting dimensions of several important common blocks, such as XOO, BOXJ, SS, and BOXH in subroutines MAIN and OTAPE to save core storage is possible. Such adjustment can be easily done through (1) updating procedure, or (2) changing common-block dimensions in those four subroutines and linking those updated members with the existing load module. To accomplish this procedure, let us introduce the functions of these common blocks and subroutines.

\section{Changes of Common Blocks for Saving Computer Storage}

\section{a. CØMMфN/XOO/}

Consisting variables for analyses involving hydrodynamics, coolant-vessel interaction, rigid cell boundaries, above-core hydrodynamics.

\section{b. $\mathrm{C} \emptyset \mathrm{MM} \phi \mathrm{N} / \mathrm{BOXJ} /$}

Consisting variables for fluid-structure interaction with deformable internals.

\section{c. $\mathrm{CQMM \emptyset N/SS/}$}

Consisting variables for cover jump and sodium spillage analysis which involves quasi-Eulerian irregular cells underneath the reactor cover. 


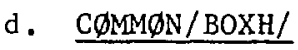

Consisting variables of marker particles involving outflow in the sodiumspillage analysis.

\section{e. CØMM}

Consisting variables for structural dynamic calculation with MAXQ $=9000$ specified internally in subroutine MAIN.

\section{Adjustment of Subroutines Needed for Saving the Computer Storage}

a. MAIN: presets dimensions of important variable and parameter in several common blocks; common blocks needed to be adjusted are XOO, BOXJ, SS, BOXH, and ALLOC, as well as the value of MAXQ.

b. OTAPE: writes or reads tapes for the purpose of continuation of a run; common blocks needed to be adjusted are XOO, BOXJ, BOXH. Note that adjustment of these common blocks are needed only if continuation of a computational run is involved.

\section{Limitations}

\section{Number of}

Eulerian cells

Cells in the radial direction

Cells in the axial direction

Marker particles

Data points per run for each CALCOMP display

Different plots for CALCOMP display

Data points on pressure-volume ratio $\left[\mathrm{p} \sim\left(\frac{\mathrm{V}}{\mathrm{V}_{\mathrm{o}}}\right)\right]$ curve

Data points on pressure-time $(P-t)$ curve

Pressure sources

Rigid obstacles

Rigid thin membrane (or plate) located at cell
Not to Exceed

420

30

100

4000

1000

100

150

150

2

350

100 


\begin{tabular}{lc}
\multicolumn{1}{c}{ Number of } & Not to Exceed \\
Eulerian cells describing the source regions & 100 \\
Eulerian cells for perforated internal structures & 100 \\
Load lines or interfaces of fluid and deformable & 10 \\
structures & 100 \\
Points, segments & 100 \\
Nodes, elements & 15 \\
Stress-strain coordinates for holddown bolts & 5 \\
Piecevise linear segments for stress-strain curve & 20 \\
of each thin shell &
\end{tabular}

The program has other limitations. These are (1) source region must be greater than one Eulerian cell, (2) marker particle must be used whether the flow is confined or involves a free surface, (3) slug impact can be analyzed only in the positive $z$ direction, (4) the flow must be laterally confined, and (5) deformation of the internal structure must be small.

The limitation on the deformations of the reactor interrals needs further explanation. Note that the ICECO code uses Eulerian coordinates in the fluid calculations. These coordinates are fixed in the space. In reactor containment the reactor vessel and internal structures are not rigidly fixed in space. As they displaced under the applied pressure loads, their boundaries will intersect the Eulerian grid lines, creating irregular cells. The treatment of the irregular cells at the fluid-strui-ure interface is very complex. Particularly, for the deformable internals the analysis usually involves multivalued field variables near the structures. Since treatment of reactor internals with large displacement is a formidable task to accomplish, present program can ouly treat small displacements of the reactor internals. 


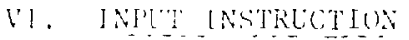

The input data cards consists ui twe parts: (1) ivdrodynanics, and (2) struc-

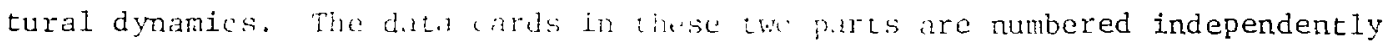

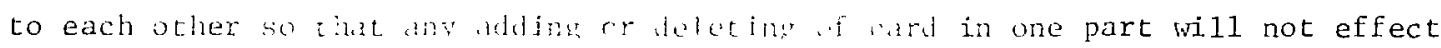
the sequence of tik wtiker.

A. Hydrodymanis Lnpu!

Note: The futawim ard types are reiulde lis inpul information to the pro-

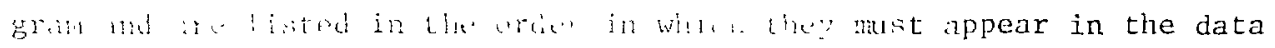
duck.

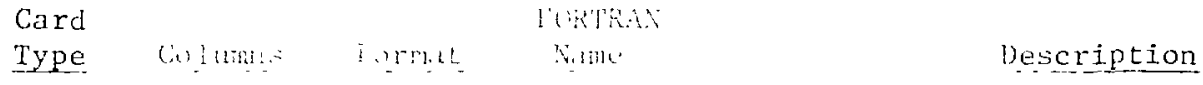

1

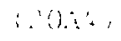

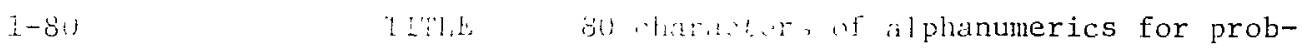
tom idcintilingion. Colum 1 must be a. i : ak.

2 (i. (1)

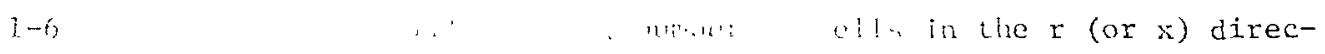
: i, in la in: tis boundary cells for

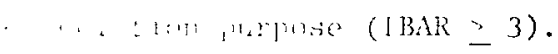

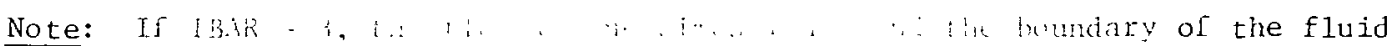

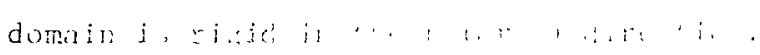

$7-12$

i. . . . ils in the $z$ (or y) direc-

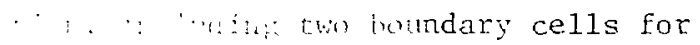

$13-18$

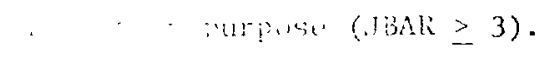
$\therefore$ wing dimensions of field : milti-material interaction.

Note that in this l.

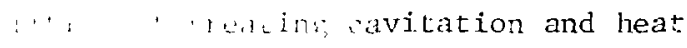

transferred aro nut it. 


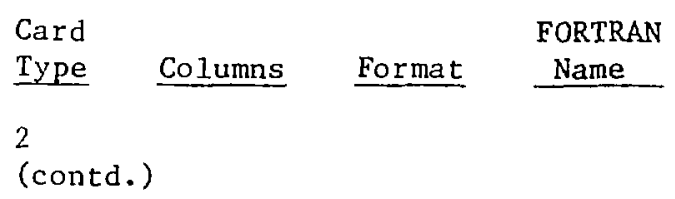

Note: See Section V for other changes needed to conserve core storage.

$2 A$

$1-6$

3
NMAT

NBAR

$1-6$
Card for multi-material interaction used only when INDMAT $=1$ on card of Type 2 . Number of different fluids (excluding the core-gas bubble and the trapped air) involved in the calculation.

Total fluid particles involved in the analysis for designing different fluids. Cards for displaying curves. Number of curves (or field variables) to be written on plot tape 2 and to be displayed later by the CALCOMP plotter. The values (or data points) for each curves are printed at the end of each run at 
Card

Type

\section{Columns Format}

(contd.)

$7-12$

4

(18I4)

$1-4$

$5-8$

$9-12$
FORTRAN

Name

\section{Description}

every NDTCLC cycle $(100 \geq N P P \geq 0)$ specified on card 11. NPP $=0$; printed values and CALCOMP plot are neglecied.

NPRS

Index for the specification of the initial value for each individual curve. NPRS = 0; the initial values of all the NPP curves are zero. NPRS $\neq 0$ : the initial value of each curve may not be zero. In this case, specify the initial value of each curve on the cards of Type 29.

Specification of the values of be displayed by the CALCOMP plotter.

$\mathrm{KXP}_{1}$ Used only when NPP > on card of Type

$\mathrm{KYP}_{1}$ 3.

$\mathrm{KZP}_{1}$
$\mathrm{KXP}_{\mathrm{m}}$, KYP ${ }_{\mathrm{m}}$, and $\mathrm{KZP}_{\mathrm{m}}$ define the values to be stored for the CALCOMP plotter: $\mathrm{m}=1, \ldots . \mathrm{NPP}(100 \geq \mathrm{NPP} \geq 0) . \operatorname{Six}$ sets of $\mathrm{KXP}_{\mathrm{m}}, \mathrm{KYP}_{\mathrm{m}}$, and $\mathrm{KZP}_{\mathrm{m}}$ values per card. Use as many cards of Type 4 as needed.

\section{Specification}

$\mathrm{KXP}_{\mathrm{m}}$ is the radial cell number $\mathrm{I}_{\mathrm{m}}$, and $\mathrm{KYP}_{\mathrm{m}}$ is the axial cell number $\mathrm{J}_{\mathrm{m}^{*}} \mathrm{KZP}_{\mathrm{m}}$ defines the values for that cell. $\mathrm{KZP}_{\mathrm{m}}=1$ : Pressure, in dynes $/ \mathrm{cm}^{2}$. ${\mathrm{K} Z \mathrm{P}_{\mathrm{m}}}=2$ : Radial velocity component, in $\mathrm{cm} / \mathrm{sec}$.

$\mathrm{KZP}_{\mathrm{m}}=3$ : Axial velocity component, in $\mathrm{cm} / \mathrm{sec}$. 
Card

Type

4

(contd.)
FORTRAN

Name

\section{Description}

$\mathrm{KZP}_{\mathrm{m}}=4:$ Here $\mathrm{KXP}_{\mathrm{m}}=100 \cdot \mathrm{J}_{\mathrm{m}}+\mathrm{I}_{\mathrm{nl}}$, and $\mathrm{KYP}_{\mathrm{m}}=100 \cdot \mathrm{J}_{\mathrm{m}}+\mathrm{I}_{\mathrm{n} 2}$. The value is the force in dynes as applied by the pressure in cells $\left(I_{n 1}, J_{m}\right),\left(I_{n I+1}, J_{m}\right)$ through $\left(\mathrm{I}_{\mathrm{n} 2}, \mathrm{~J}_{\mathrm{m}}\right)$ on the upper area of the above cells.

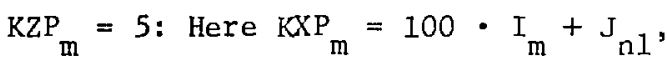
and $\mathrm{KYP}_{\mathrm{m}}=100 \cdot \mathrm{I}_{\mathrm{m}}+\mathrm{J}_{\mathrm{n} 2}$. The value is the force in dynes as applied by pressure in cells $\left(I_{m}, J_{n l}\right)$ through $\left(I_{m}, J_{n 2}\right)$ on the right-side area of the above cells.

$\mathrm{KZP}_{\mathrm{m}}=6:$ Here $\mathrm{KXP}_{\mathrm{m}}=100 \cdot \mathrm{J}_{\mathrm{m}}+\mathrm{I}_{\mathrm{nI}}$, and $\mathrm{KYP}_{\mathrm{m}}=100 \cdot \mathrm{J}_{\mathrm{m}}+\mathrm{I}_{\mathrm{n} 2}$. The value is the upward force in dynes applied on the perforated plate. The plate is oriented horizontally and is spanned from $\operatorname{cell}\left(I_{n l}, J_{m}\right)$ to $\left(I_{n 2}, J_{m}\right)$ which may have full-cell openings. $\mathrm{KZP}_{\mathrm{m}}=7$ : Pressure on the structural segment, in dynes/cm.

$\mathrm{KXP}_{\mathrm{m}}=0$ or arbitrary.

$\mathrm{KYP}_{\mathrm{m}}=$ the segment number.

$\mathrm{KZP}_{\mathrm{m}}=8$ : Radial velocity at the midpoint of the structural segment, in $\mathrm{cm} / \mathrm{sec}$.

$\mathrm{KXP}_{\mathrm{m}}=0$.

$\mathrm{KYP}_{\mathrm{m}}=$ segment number. $\mathrm{KZP}_{\mathrm{m}}=9$ : Axial velocity of the midpoint of the structural segment, in $\mathrm{cm} / \mathrm{sec}$. 
Card

Type

4

(contd.)
FORTRAN

Name

\section{Description}

$\mathrm{KXP}_{\mathrm{m}}=0$.

$\mathrm{KYP}_{\mathrm{m}}=$ segment number .

$\mathrm{KZP}_{\mathrm{m}}=10:$ Radial displacement of the structural node point, in $\mathrm{cm}$.

$\mathrm{KXP}_{\mathrm{m}}=0$.

$\mathrm{KYP}_{\mathrm{m}}=$ node point number .

$\mathrm{KZP}_{\mathrm{m}}=11$ : Axial displacement of the structural node point, in $\mathrm{cm}$.

$\mathrm{KXP}_{\mathrm{m}}=0$.

$\mathrm{KYP}_{\mathrm{m}}=$ point number .

$\mathrm{KZP}_{\mathrm{m}}=12:$ Circumferential strain of

the structural node.

$\mathrm{KXP}_{\mathrm{m}}=$ the component number (see Fig. 68).

$\mathrm{KYP}=$ the element number.

$\mathrm{KZP}_{\mathrm{m}}=13:$ Meriendianal strain of the

structural element, in \%.

$\mathrm{KXP}_{\mathrm{m}}=$ the component number (see Fig. 68).

$\mathrm{KYP}_{\mathrm{m}}=$ the element number.

$\mathrm{KZP}_{\mathrm{m}}=14$ : Total net force on the radial

shield core barrel simulated by the rigid

obstacles, in dynes $/ \mathrm{cm}^{2}$. Here $\mathrm{KXP}=100$

- $\mathrm{I}_{\mathrm{m}}+\mathrm{J}_{\mathrm{n} 1}$ and $\mathrm{KYP}_{\mathrm{m}}=100 \cdot \mathrm{I}_{\mathrm{m}}+\mathrm{J}_{\mathrm{n} 2} \cdot$ The value is the outward force in cells ( $I_{m-1}$, $\left.\mathrm{J}_{\mathrm{n} 1}\right)$ through $\left(\mathrm{I}_{\mathrm{m}-1}, \mathrm{~J}_{\mathrm{n} 2}\right)$ subtract the inward force in cells $\left(\mathrm{I}_{\mathrm{m}+1}, \mathrm{~J}_{\mathrm{nl}}\right)$ through $\left(I_{m+1}, J_{n 2}\right)$. Note that $I_{m}$ denote the radial cell number of the radial sheild. $\mathrm{KZP}_{\mathrm{m}}=15$ : Axial position of the marker particle, in cm. $\mathrm{KYP}_{\mathrm{m}}=$ the particle number. $\mathrm{KZP}_{\mathrm{m}}=16:$ Total mass of fluid spillage through penetration opening on the vessel cover, in gm. 
Card

Type
FORTRAN

Name

\section{Description}

4

(contd.)

\footnotetext{
$\mathrm{KXP}_{\mathrm{m}}=$ the radial cell number with the opening.

$\mathrm{KZP}_{\mathrm{m}}=17:$ Total mass of fluid spillage through vessel-head junction, in gm. $\mathrm{KZP}_{\mathrm{m}}=18$ : Total force acting on the movable head, in dynes.

$\mathrm{KZP}_{\mathrm{m}}=19$ : Total force in the hold-down bolts if the head is movable, in dynes. $\mathrm{KZP}_{\mathrm{m}}=20$ : Axial displacement of the movable head, in $\mathrm{cm}$.

$\mathrm{KZP}_{\mathrm{m}}=21$ : Velocity of the movable head, in $\mathrm{cm} / \mathrm{sec}$.

$\mathrm{KZP}_{\mathrm{m}}=22$ : Acceleration of the movable - head, in $\mathrm{cm} / \mathrm{sec}^{2}$.

$\mathrm{KZP}_{\mathrm{m}}=23$ : Pressure in the quasi-Eulerian cell generated by the movable head, in dynes $/ \mathrm{cm}^{2}$. $\mathrm{KZP}_{\mathrm{m}}=24$ : Radial velocity in the quasiEulerian cell defined at right-hand boundary, in $\mathrm{cm} / \mathrm{sec}$.

$\mathrm{KZP}_{\mathrm{m}}=25$ : Axial velocity in the quasiEulerian cell defined at upper rell boundary, in $\mathrm{cm} / \mathrm{sec}$.

$\mathrm{KZP}_{\mathrm{m}}=26$ : Impulse acting on the penetration opening of the vessel head, in dyne$\mathrm{sec} / \mathrm{cm}^{2}$.

$\mathrm{KXP}_{\mathrm{m}}=$ the radial cell number having the opening.

$\mathrm{KZP}_{\mathrm{m}}=27:$ Density in the quasi-Eulerian cell, in $\mathrm{gm} / \mathrm{cm}^{3}$.

$\mathrm{KXP}_{\mathrm{m}}=$ the radial cell number.

$\mathrm{KZP}_{\mathrm{m}}=28$ : Velocity in the penetration opening, in $\mathrm{cm} / \mathrm{sec}$.
} 

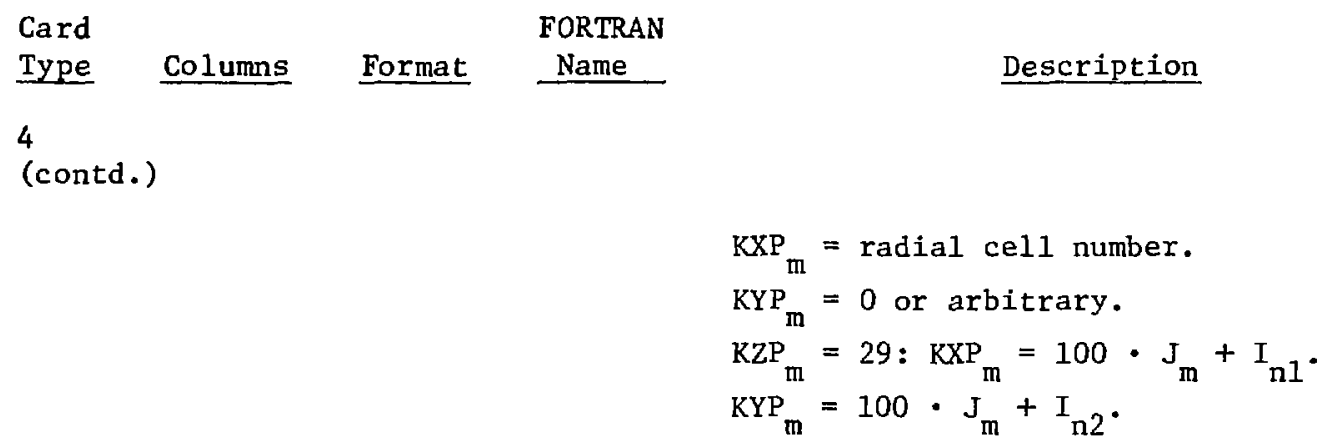

Note: (a) Cards of Type 4 are not needed when NPP $=0$ is specified on card of Type 3.

(b) If NPRS $\neq 0$ on card of Type 3 , the initial vaiue of each curve may be different from zero and hence should be specified on cards of Type 29.

(c) Calculations of the circumferential stfain (KZP = 12) and the meriendianal strain $(K Z P=13)$ are done in the finite element program. See Fig. 68 for determination of the component number. 
Card

Type

5

$1-6$
FORTRAN

Format

(2I6)

IOUT
Description

Parameter to determine restartcapability usage of auxiliary tapes 8 and 9.

IOUT $=0:$ Tapes 8 and 9 are not used. IOUT $=1:$ After the computation is terminated, the program writes the output data on a binary tape 8 so that the problem may be continued later. IOUT $=2:$ Continuation of the problem from a previous run. Program reads the input data from a binary tape 9 . IOUT $=3:$ Program combines both IOUT $=$ 1 and IOUT $=2$ capabilities. Program reads the input data from a binary tape 9 and also writes the output data on a binary tape 8 for later continuation.

Note: For IOUT $=2$ or 3 , the input to the program is on tape 9; thus, only cards of Type 1-20 are required.

$7-12$

$13-18$

$19-24$
IZIP

IFLMCV

INDPEF
Index for choosing the differencing scheme. IZIP $=0:$ ZIP differencing.

IZIF $=1:$ Complete donor-cell differencing.

IZIP = 2: Partial donor-cell differencing.

Index for plotting the reactor cover. IFLMCV $=0$ : Reactor cover is not plotted. IFLMCV $=1$ : Reactor cover is plotted.

Index for analyzing perforated structures by the control-volume method. INDPEF $=0$ : No perforated structure. INDPEF $=1:$ (not available). With perforated structure analyzed by the 
Card

Type

Columns

Format

5

(contd.)
FORTRAN

Name

\section{Description}

control-volume method. Provide hydrodynamic information on cards of Type 49-53.

25-30

JHEAD

Axial cell number at which the upper cell boundary coincides with the bottom of the reactor cover. JHEAD $=$ JBAR-1 for rigid cover.

$31-36$ NCONVG Number of iterations the solution is forced to be converged af ter NCYCVG cycle of calculation (for debugging purpose).

NCONVG $=0$ : No limitation on the number of iteration.

$37-42$

ISTATE

Index for indicating type of fluid and $i$ ts equation of state involved in the analysis.

ISTATE = 0: Water or sodium.

ISTATE $=1:$ Gas.

$43-48$

MINITR

Minimum number of iterations for improving the solution of the coupled fluidstructure probiem.

MINITR $=0$ : No minimum iterations. MINITR > 0: Minimum number of iterations is MINITR. Input card 5A required.

NCYCVG Cycle number after which the solution is assumed to be converged after NCONVG iteration (for debugging purpose).

$5 A$ (1E12.4)

$1-12$

VDIF

Velocity discrepancy associated with MINITR spectfied on card of Type 5. 
Card
Type Columas Format $\begin{gathered}\text { FORTRAN } \\ \text { Name }\end{gathered}$

$5 \mathrm{~A}$

(contd.)

\section{Description}

In the boundary cell if $\left|\overline{\mathrm{V}}_{\mathrm{p}}-\overline{\mathrm{V}}_{\mathrm{b}}\right|$ is greater than VDIF the iteration will continue at least MINITR times to ensure the accuracy of the boundary treatment.

Note: Card of Type 5A is not required if MINITR $=0$ specified on card Type 5 . 6 (1216) Parameter card.

$1-6$ KY

Number of marker particles initially assigned to each full cell (KY > 0).

Note: Particles located at the upper and right edges of the cell $(i, j)$ will belong to $\operatorname{cell}(i, j)$.

$7-12$

$13-18$

$19-24$
NLAST

IDT

IVIS
Stop cycle; allows the problem to be terminated after stop cycle.

Index for selecting the time step. IDT $=1$ : Constant time step is used. This time step should be specified on card of Type 18.

IDT = 2: 12 varied time steps are used in the calculation. These steps should be specified on cards of Type 16 and 17 , and will be changed automatically at 12 cycles (NCL1, NCL2, ..., NCL12) prescribed on cards of Type 15. Viscosity index (a parameter for selecting the viscosity coefficients $\mu$ and $\lambda$ of the fluid used in the calculation). IVIS $=0$ : Viscosities $\lambda$ and $\mu$ are neglected.

IVIS $=1: \lambda=0$, real value of $\mu$ is used. The value of $\mu$ is specified on card of Type 19. 
Card

Type

Colunns Format

6

(contd.)
FORTRAN

Name

\section{Description}

IVIS $=2: \mu=0$, artificial coefficient $\lambda$ is used.

IVIS $=3: \lambda=0$, artificial coefficient

$\mu$ is used.

IVIS $=4: \mu=0$, real value of $\lambda$ is used and should be specified on card of Type 19.

IVIS $=5:$ Real values of both $\lambda$ and $\mu$ are used, and these values should be specified on card of Type 19.

IVIS $=6:$ Artificial viscosities $\lambda$ and $\mu$ are used.

Index controlling mass-diffusion coefficient.

ITAU $=0$ : Mass-diffusion coefficient $\tau$ is taken to be zero.

ITAU = 1: Constant value of $\tau$ is used. The value should be specifled on card of Type 19.

ITAU = 2: Artificial mass-diffusion coefficient is considered.

Number of cells (or number of regions, $|\mathrm{NP}|$, in case of $\mathrm{NP}<0$ ) with initial pressure, velocities, internal energy, etc., input individually. Thus, in the program the uniform initial conditions (pressure, velocities, and specified internal energy specified on card of Type 22) In these NP cells will be Overridden by the nonuniform initial conditions provided on cards of Type 30 or $30 \mathrm{~A}$. 
Card

Type

Columans Format

6

(contd.)

$37-42$

43-48

49-54
FORTRAN

Name

\section{Description}

INDOUT

Outflow (fluid spillage) controlling

index.

INDOUT $=0:$ No fluid spillage.

INDOUT = 1: Fluid spillage through

one-cell opening at the upper contain-

ment wall immediate below the vessel

head.

INDOUT $=2:$ Fluid spillages through both penetrations and the side opening at the vessel-head junction. In this case, input data for penetrations and side opening are needed to be specified on cards of Type 42-46.

INDBC Index controlling outflow (fluid spillage) boundary condition.

INDBC $=0:$ Outflow boundary condition is not specified; set INDOUT $=0$.

INDBC $=1$ : Downstream of the outflow boundary is assumed to be a pressure free and frictionless region; used when INDOUT $>0$.

INDBC > 1: Not available.

INDHT is generated by the heat transfer mechanism (such as fuel-coolant interaction). INDHT = 0 : Source pressure is not generated by the heat transfer mechanism. INDHT $\neq 0$ : (not available?. Source pressure is generated by the heat-transfer mechanism. 


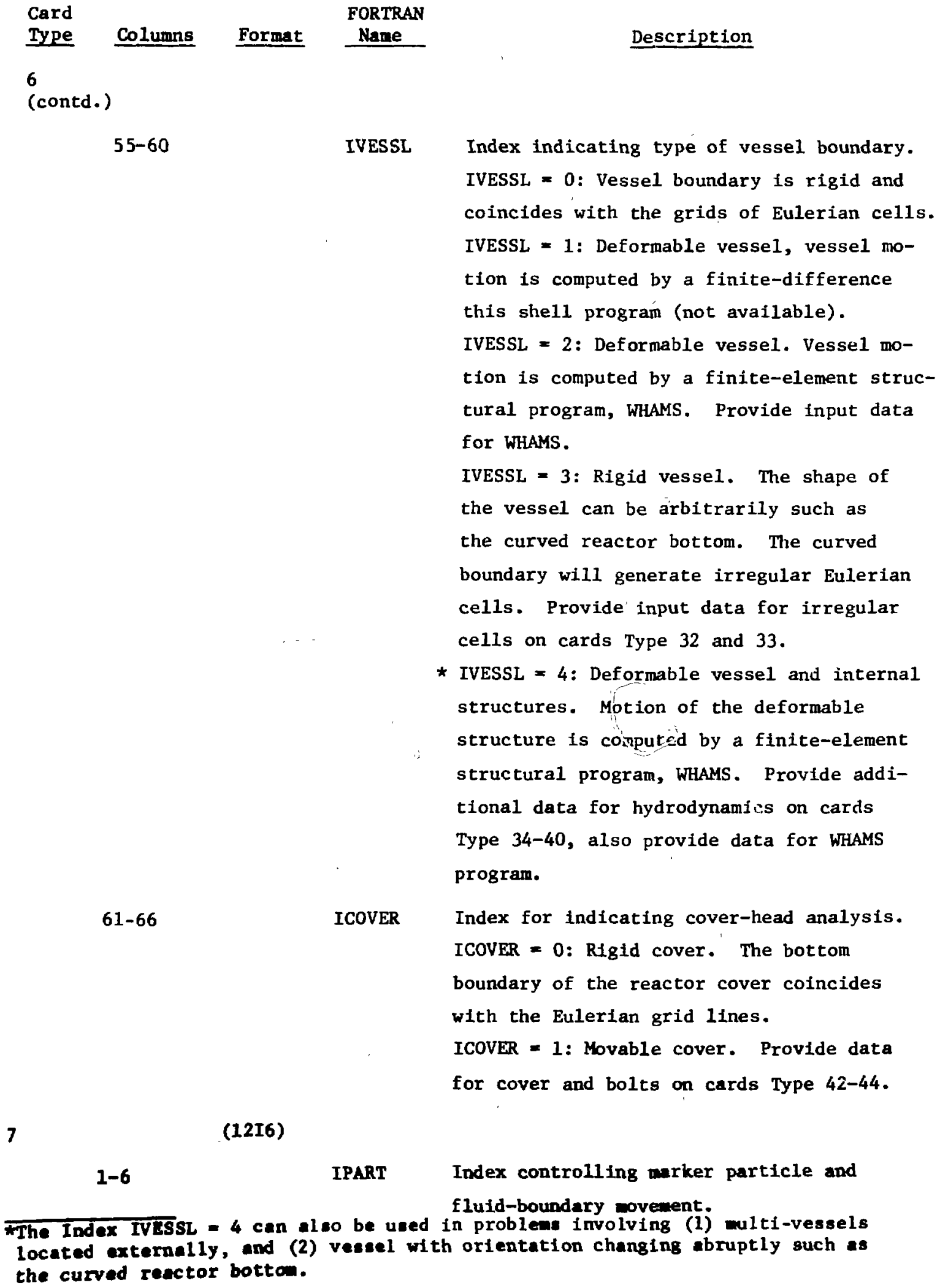


Card

Type

Columns Format

(contd.)
FORTRAN

Name

글

)

$7-12$

$13-18$

$19-24$

$25-30$

31-36

$37-42$

\begin{tabular}{|c|c|c|}
\hline $7-12$ & INDPCT & $\begin{array}{l}\text { Slug-Impact index. } \\
\text { INDPCT = } 0: \text { No slug impact. } \\
\text { INDPCT }=1: \text { With slug impact. }\end{array}$ \\
\hline $13-18$ & KIMP & $\begin{array}{l}\text { Number of marker particles contacting } \\
\text { vessel cover required to indicate the } \\
\text { beginning of slug impact (suggest } \\
\text { KIMP }=1 \text { ). }\end{array}$ \\
\hline $19-24$ & INDCOR & $\begin{array}{l}\text { Number of source regions (or free sur- } \\
\text { face plus interface) involved in the } \\
\text { analysis. The first source region is } \\
\text { the core-gas bubble; the second source } \\
\text { region is the cover-gas region ( } 0< \\
\text { INDCOR } \leq 2 \text { ). } \\
\text { INDCOR = } 1 \text { : Only reactor core considered } \\
\text { as the source. }\end{array}$ \\
\hline $25-30$ & MPA & $\begin{array}{l}\text { Number of particles used to define the } \\
\text { boundary of the core-gas bubble (or the } \\
\text { first source region). }\end{array}$ \\
\hline $31-36$ & MPB & $\begin{array}{l}\text { Number of particles used to specify the } \\
\text { free-surface position (that is, the in- } \\
\text { terface between the cover-gas region } \\
\text { and the sodium pool). }\end{array}$ \\
\hline $37-42$ & INTEG & $\begin{array}{l}\text { Index for calculating the volume of the } \\
\text { core-gas bubble. } \\
\text { INTEG = } 1 \text { : Calculating bubble volume with } \\
\text { respect to the } r \text { axis. }\end{array}$ \\
\hline
\end{tabular}

IPART $=0:$ Fluid movement is neglected. This approach is sometimes used in the pipe flow.

IPART $=1:$ Fluid movement is considered.

Slug-Impact index.

Number of marker particles contacting vessel cover required to indicate the beginning of slug impact (suggest $\operatorname{IMP}=1)$

\section{Description}


Card

Type

Column

7

(contd.)
$43-48$

$49-54$

$55-60$
FORTRAN

Name

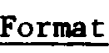

Description

INTEG $\neq 1$ : Calculating bubble volume with respect to the $z$ axis.

INDMPA

Index for film display of marker particles around the core.

INDMPA $=0:$ Marker particles defining

the core-gas bubble are not connected by straight lines.

INDMPA = 1: Marker particles defining the core-gas bubble are connected by the straight lines through the FILM subroutine.

ISHALW

Index for excursion geometry.

ISHALW $=0$ : Deep excursion in which regions of the core-gas bubble and the upper cover gas can be separated by a value of YMAX. In this case, specify MAX on card of Type 12 . ISHALW = 1: For shallow-coolant excurtion in which the core-gas bubble and upper cover-gas regions cannot be separated by a prescribed value.

INDKT Index indicating interfacial boundary condition during the slug impact. INDKT $=0$ : Particles on the coolant free surface once contact with the cover will remain in contact but can slide tangentially.

INDKT = 1: (not available). Particles contact with the cover can separate from the cover. 
Card FORTRAN

Type Columns Format Name

\section{Description}

7

(contd.)

$61-66$

IEXPN

Index indicating the effect of the radiai

shield on the core-gas bubble expansion;

used when core particles move to the right-

hand side of the radial shield (such as in

the SRI experiments).

IEXPN $=0:$ Bubble-volume calculation is

not effected by the radial shield.

IEXPN $=1:$ Bubble-volume calculation is

effected by the rigid radial shield.

Supply information of the radial shield

and core particles on card of Type 28D

for correct volume computation.

IEXPN $=2:$ Bubble-volume calculation is

effected by tie flexible radial shield.

Supply information on card of Type $28 \mathrm{E}$

for correct volume computation.

$67-72$

IFLAG

Index indicating the flagging scheme used

at the free surface and the bubble-coolant

interface.

IFLAG $=0$ : Detailed flagging scheme similar

to the one used at the curved vessel bottom.

IFLAG = I: Conventional flagging scheme

such as the one used in the MAC method.

Note: (a) When IFLAG $=0$, the first and last marker particles of the core-

gas bubble must be located on the symmetrical axis.

(b) For the case of strong source pressure and large curvature at

the bubble-coolant interface IFLAG $=0$ may give better results.

(c) See Section $V$ for descriptions of the flagging schemes.

8

(5I6)
Source card.

$1-6$

INDPT 
Card

Type

Columens Format

8

(contd.)
$7-12$

13-18
FORTRAN

Nome

\title{
Description
}

\begin{abstract}
INDPT $=0:$ Source pressures are calculated by pressure-volume $(p-v)$ relationship. INDPT $=1$ : Source pressures are determined by the pressure-time $(p-t)$ relationship.
\end{abstract}

INDPTA Index to determine the $p-t$ relationship at the core-gas bubble, or source region at the lower end of the fluid region. INDPTA $=0$ : Gas bubble is subjected to zero pressure. INDPTA $=1$ : Gas bubble is subjected to a constant-pressure PRESS specified on card of Type 23.

INDPTA $=2:$ Gas bubble is subjected to a pressure $P=F_{1}(t)$, with number of data points specified by $\mathrm{KCA}$, and the corresponding pressure and time values prescribed on cards of Type 9.

INDPTA = 3: Gas bubble is subjected to a pressure calculated from $\mathrm{pV}^{\gamma}=\mathrm{c}$. Specify Initial pressure, energy, and gas exponent on cards of Type 23.

INDPTA $=4:$ Gas bubble is subjected to a pressure $P=F_{1}\left(V / V_{0}\right)$, with number of data points specified by KCA, and the corresponding pressure and volume-ratio $\left(\mathrm{V} / \mathrm{V}_{\mathrm{o}}\right)$ values prescribed on cards of Type 9.

INDPTB Index to indicate the $p-t$ relationship at the cover-gas region or at the upper end of the fluid region. INDPTB = 0 : Cover-gas region is subjected to a zero pressure. 
Card

Type
FORTRAN

Name

\section{8}

(contd.)

$\mathrm{KCA}$

$25-30$

(8F9.0)

\section{Format}

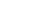
.

\section{Description}

INDPTB $=1:$ Cover-gas region is subjected to a constant pressure PRESSB specified on card of Type 24.

INDPTB $=2$ : Cover-gas region is subjected to a pressure $p=F_{2}(t)$, with number of data points specified by $\mathrm{KCB}$, and the corresponding pressure and time values prescribed on cards of Type 10. INDPTB $=3$ : Cover-gas region is subjected to a pressure calculated from $\mathrm{PV}^{\gamma}=\mathrm{C}$, specify initial, pressure energy, and gas exponent on cards of Type 24.

INDPTB = 4: Gas-gas (or second source) region is subjected to" a pressure $\mathrm{p}=$ $\mathrm{F}_{2}\left(\mathrm{~V} / \mathrm{V}_{\mathrm{o}}\right)$, with number of data points specified by $\mathrm{KCB}$, and the corresponding pressure and volume-ratio $\left(\mathrm{V} / \mathrm{V}_{\mathrm{o}}\right)$ values prescribed on cards of Type 10.

Number of data points on the $F_{1}(t)$ [or $\left.\mathrm{F}_{1}\left(\mathrm{~V} / \mathrm{V}_{\mathrm{o}}\right)\right]$ curve applied at the core-gas bubble or at the lower end of the sluid region $(0 \leq \mathrm{KCA} \leq 150)$. Number of data points on the $F_{2}(t)$ [or $\left.\mathrm{F}_{2}\left(\mathrm{~V} / \mathrm{V}_{\mathrm{o}}\right)\right]$ curve applied at the upper gas gap or the upper end of the fluid region $(0 \leq \mathrm{KCB} \leq 150)$.

$\left.\begin{array}{lc}1-9 & \mathrm{PPA}_{1} \\ 10-18 & \mathrm{VVA}_{i}\end{array}\right\}$

$P_{i}$ and $T_{i}(i=1,2, \ldots)$ are the pressure and time (or volume ratio) values, respectively. These values are taken from the data points of pressure-versus-time (or 


\section{Card , FORTRAN \\ Type Columns Format Name \\ 9 \\ (contd.)}

etc.

\section{Description}

volume ratio) curve specified for the core-gas bubble region.

Note: (a) There are KCA (see card Type 8) sets of pressure and time (or volume ratio) input, each card consists of 4 sets of them.

(b) The times (or volume ratios) on cards of Type 9 are in increasing "order.

(c) Cards of Type 9 are required if either INDPTA $=2$ or 4 specified on card Type 8 .

10

$(8 \mathrm{F9} .0)$

$\left.\begin{array}{lc}1-9 & \mathrm{PPB}_{i} \\ 10-18 & \mathrm{VVB}_{i}\end{array}\right\}$
$P_{i}$ and $T_{i}(i=1,2, \ldots)$ are the pres- sure and time (or volume ratio) values, respectively. These values are taken from the data points of pressure-versus- time (or volume ratio) curve specified for the cover-gas region.

Note: (a) There are $\mathrm{KCB}$ (see card Type 8) sets of pressure and time (or volume ratio) input. Each card consists of 4 sets of them.

(b) The times (or volume ratios) on cards of Type 10 are in increasing order.

(c) Cards of Type 10 are required if either INDPTB $=2$ or 4 is specified on card Type 8.

Frequency card.

Frequency of film output. NDTP $=0:$ No film output. NDTP $\geq 1$ : Marker-particle configuration is displayed every NDTP cycle.

Frequency of output of physical variables for all Eulerian cells. These variables include $u, v, c, p$, and cell flag F. 
Card

Type

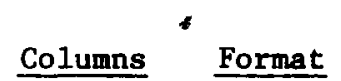

11

(contd.)
FORTRAN

Name

$13-18$

$19-24$

$25-30$

$31-36$

NDTPP

NDTCLC

NDTGEN

NGRID

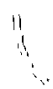

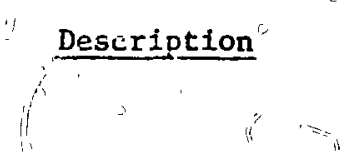

NDTCP $=0:$ No variable output.

NDTCP $\geq 1:$ Cell-variable output every NDTCP cycle.

Frequency of the printout of markerparticle positions. The printout includes the numbering of the particles, and the radial and axial positions of each marker particle.

NDTPP $=0:$ No particle-position printout. NDTPP $\geq 1:$ Particle-position printout every NDTPP cycle.

Frequency of data points to be plotted on each curve. Maximum number of data points for each curve is limited to 1000. Thus, if the computing cycles exceed 1000, frequency is needed so that each curve is made of 1000 data points or less. NDTCLC $=0:$ CALCOMP plot is neglected. NDTCLC $\geq 1:$ Each curve is made of values obtained from every NDTCLC cycle.

Frequency of general output. The printout includes the source informations, cycle number, time, iteration number, viscosities, and etc.

NDTGEN $=0$ : No general printout. NDTGEN > 0: General/printout every NDTGEN cycle.

Frequency to omit the plotting of grid lines on the film output. - HGRID must be multiple value of NDTP. 
Card

Type

11

(contd.)
FORTRAN

Name

\section{Description}

< 1: Program reset NGRID = NDTP.

NGRID > $I$ : Grid lines are omitted at

every NGRID cycle.

$37-42$

NDTSTR

Frequency of output of the structural dynamics.

NDTSTR $=0:$ No printout.

NDTSTR $>0$ : Printout at every NDTSTR

cycle (not available).

$43-48$

49-54

$55-60$

(6E12.4)

1-12

$13-24$

PC
NDTFLG

NDTINT

NDTSSP

DZ

Frequency of output for the boundary

cell flag.

NDTFLG $=0:$ No printout.

NDTFLG > 0: Printout at every NDTFLG

cycle (not available).

Frequency for printout hydrodynamics of deformable internal structures.

NDTINT $=0$ : No printout.

NDTINT > 0: Printout at every NDTINT

cycle (not aviliable).

Frequency for printout hydrodynamics of sodium spillage.

NDTSSP $=0:$ No printout.

NDTSSP > 0: Printout at every NDTSSP

cycle (not: available).

DR

$\delta r($ or $\delta x$ ), the cell size in the $r$ (or $x$ )

direction, in centimeters.

$\delta z$ (or $\delta y$ ), the cell size in the $z$ (or $y$ )

direction, in centimeters.

Problem-geometry parameter. 
Card

Type

Columns

Format

12

(contd.)
FORTRAN

Name

\section{Description}

$P C=0:$ Calculation performed in the cylindrical coordinates with axial symmetry (in $\mathrm{r}-\mathrm{z}$ plane). $\mathrm{PC}=1.0:$ Calculation performed in the plane coordinates (in $\mathrm{A}^{-y}$ plane).

PROB

Parameter to determine flow characteristic. PROB $=1.0:$ Incompressible-flow calculation; the equation of state is bypassed in the analysis.

PROB $=2.0:$ Compressible-flow calculation.

$49-60$

CONST

$\rho_{0}$, density of the fluid at the standard conditions, in $\mathrm{g} / \mathrm{cm}^{3}\left(\rho_{0}>0\right)$.

$61-72$

YMAX

A maximum value in the $z$ (or $y$ ) direc-

tion where the maximum axial position of the bubble boundary is not expected to exceed during the expansion in the computing run, in $\mathrm{cm}$. This height is used to differentiate the region of core-gas bubble and the cover gas. Thus, all empty cells and surface cells having axial coordinates $\left(z_{i, j}\right.$ or $\left.y_{i, j}\right)$ smaller than YMAX will be assumed to be the gasbubble region. All empty cells and surface cells having the axial coordinates greater than YMAX will be assumed to be the cover-gas region.

(I6) inftial gas bubble where pressure calculated is the source pressure $\{100>$ NSOUR $>0$ ). 
Card FORTRAN

Type Columns Format Name Description

13

(contd.)

Note: Gas bubble must be greater than one cell size.

14

(12I6)

$\begin{array}{lll}1-6 & \text { KSOUX }_{i} & I_{i} \text { and } J_{i}(i=1,2, \ldots) \text { define, respec- } \\ 7-12 & \text { KSOUY }_{i} & \text { tively, the radial and axial cell number } \\ & & \text { used to represent the initial position } \\ & & \text { of the core-gas bubble. }\end{array}$

Use as many cards of Type 14 as required; $i=1, \ldots$, NSOUR $(0<$ NSOUR $\leq 100)$.

15

(12I6)

$\begin{array}{ccl}1-6 & \text { NCL1 } & \text { NCL1, NCL2, } \ldots, \text { NCL12 are } 12 \text { prescribed } \\ 7-12 & \text { NCL2 } & \text { computational cycles at which the time } \\ \text { - } & \text { step }(\delta t) \text { is expected to be changed for } \\ \text { - } & \text { the numerical integration. The } 12 \text { time } \\ \text { - } & \text { steps (DLT1, .., DLT12) are specified } \\ 67-72 & \text { NCL12 } & \text { on the cards of Type } 16 \text { and } 17 .\end{array}$

Note: (a) This card is omitted if IDT $=1$ is specified on card of Type 6 .

(b) To start fluid motion, it is suggested that NCL1 $\geq 5$. In this case, the program will use a time step of $0.1 \times 10^{-6} \mathrm{sec}$ for the first five computation cycles.

16

(6E12.4)

$\begin{array}{cc}1-12 & \text { DLT1 } \\ \cdot & \cdot \\ \cdot & \cdot \\ 61-72 & \text { DLT6 }\end{array}$
Time steps, in seconds; $\delta t_{i}$ represents the corresponding time steps expected to be changed at the respective cycle $\mathrm{NCL}_{1}$ specified on card of Type 15. Thus the program will use $0.1 \times 10^{-6}$ sec if $\mathrm{NCL}_{1}>$ cycle $>1 ; \delta t_{1}$ if $\mathrm{NCL}_{2}>$ cycle $>\mathrm{NCL}_{1} ; \delta \mathrm{t}_{2}$ if $\mathrm{NCL}_{3}>$ cycle $>\mathrm{NCL}_{2}$; ..; $\delta \mathrm{t}_{6}$ if $\mathrm{NCL}_{7}>$ cycle $>\mathrm{NCL}_{6}$.


Card Type

17

\begin{tabular}{|c|c|c|}
\hline Columins & Format & Name \\
\hline & $(6 E 12.4)$ & \\
\hline $1-12$ & & DLT7 \\
\hline $13-24$ & & DLT8 \\
\hline - & & - \\
\hline - & & - \\
\hline - & & - \\
\hline $61-72$ & & DLT12 \\
\hline
\end{tabular}

Description

Time steps, in seconds, $\delta t_{i}$ represents the corresponding time steps expected to be changed at the respective cycles $\mathrm{NCL}_{i}(i=7, \ldots, 12)$ specified on cards of Type 15. Thus the program will use $\delta t_{7}$ if $\mathrm{NCL}_{8}>$ cycle $>\mathrm{NCL}_{7} ; \delta t_{8}$ if $\mathrm{NCL}_{9}$ $>$ cycle $>\mathrm{NCL}_{8} ; \ldots ; \delta \mathrm{t}_{12}$ if cycle > $\mathrm{NCL}_{12}$.

Note: (a) Cards of Type 16 and 17 are omitted if IDT $=1$ is specified on card of Type 6 .

(b) The size of the time step is also controlled by the stability criteria. If any time step prescribed on the cards of Type 16 and 17 is greater than the one satisfying the stability criteria, the program will use the step calculated by the stability criteria.

(c) For the compressible-flow problem, it is suggested that the input time steps should not exceed about $10^{-4} \mathrm{sec}$. Also, time steps should increase gradually.

(3E12.4)

$1-12$

$13-24$

25-36

$37-42$
EPS

EPSS

DTCON

TYPE $\varepsilon$, convergence criterion for the iteration of pressure field. Usually $\varepsilon$ is of the order of $5 \times 10^{-4}$.

$\mathrm{P}_{\text {min }}$, the minimum allowable pressure in the flutd region (usually zero), in dynes $/ \mathrm{cm}^{2}$.

$\delta t$, the constant time step, in seconds, used only when IDT $=1$ is defined on card of Type 6. (If IDT $\neq 1$, let DTCON $=0.1 \times 10^{-6} \mathrm{sec}$ ).

Parameter for indicating rigid obstacle option. 


\begin{tabular}{|c|c|c|c|}
\hline $\begin{array}{l}\text { Card } \\
\text { Type }\end{array}$ & Columns & Format & $\begin{array}{l}\text { FORTRAN } \\
\text { Name }\end{array}$ \\
\hline (con & & & \\
\hline
\end{tabular}

TYPE $=0.0$ : No rigid obstacle in the computing region.

TYPE $=1.0:$ With rigid obstacles in the computing region.

Note: (a) If TYPE $=1.0$ input data concerning number of cells occupied by the obstacles and perforated holes generated by the arrangement of obstacles should be specified on cards of Type 25, 26, and 27.

(b) Each obstacle occupies one full cell, its boundaries coincide with grid lines.

19

$$
\text { (6E12.4) }
$$

VISR

$\mu$, second (dynamic) viscosity coefficient of the fluid, in dyne-sec/cm ${ }^{2}$. A real number.

$13-24$

VOVISR

$\lambda$, first (volumetric) viscosity coefficient of the fluid, in dyne-sec/ $/ \mathrm{cm}^{2}$. Also a real number.

25-36 TAU $\tau$, mass-diffusion coefficient (usually zero).

$\phi$, weighting constant for the pressure field in the finite-difference momentum equation. $(1.0 \geq \phi>0$; usually $\phi=1.0)$. $\theta$, weighting constant in the finitedifference mass equation. $(1.0 \geq \theta>$ 0 ; usually $\theta=1.0$ ).

The quotient of $\gamma$ to Prandtl number. $B$ is a constant relating the temperature change with the variation of internal energy of a heat-conducting fluid; for a non-heat-conducting fluid, $B=0$. 


\begin{tabular}{|c|c|c|c|c|}
\hline $\begin{array}{l}\text { Card } \\
\text { Type }\end{array}$ & Columns & Format & $\begin{array}{l}\text { FORTRAN } \\
\text { Neme } \\
\end{array}$ & Description \\
\hline \multirow[t]{6}{*}{20} & & (6E12.4 & & \\
\hline & $1-12$ & & GR & $\begin{array}{l}8_{r} \text {, gravity in the } r \text { or } x \text { direction } \\
\text { (either positive or negative), in dyne- } \\
\mathrm{cm} / \mathrm{sec}^{2} \text {. }\end{array}$ \\
\hline & $13-24$ & & GZ & $\begin{array}{l}g_{z} \text {, gravity in the } z \text { or } y \text { direction } \\
\text { (either positive or negative), in dyne- } \\
\mathrm{cm} / \mathrm{sec}^{2} \text {. }\end{array}$ \\
\hline & $25-36$ & & TS & $\begin{array}{l}\text { Initial starting time of problem in } \\
\text { seconds. }\end{array}$ \\
\hline & $49-60$ & & TL & $\begin{array}{l}\text { Maximum time, in seconds; allows the } \\
\text { problem to be terminated after TL sec. } \\
\text { The width of the computing region (in } \\
r \text { or } x \text { direction), in centimeters. This } \\
\text { value does not include two boundary cells } \\
{[X R=(I B A R-2) \times D R] \text {. }}\end{array}$ \\
\hline & $61-72$ & & YT & $\begin{array}{l}\text { The height of the computing region (in z } \\
\text { or y direction), in centimeters. This } \\
\text { value does not include two boundary cells } \\
{[\mathrm{YT}=(\text { IBAR }-2) \times \mathrm{DZ}] \text {. }}\end{array}$ \\
\hline \multirow[t]{2}{*}{21} & & (1I6) & & \\
\hline & $1-6$ & & $\mathbf{N M}$ & $\begin{array}{l}\text { Number of marker-particl: sets to be } \\
\text { created. For each set of marker parti- } \\
\text { cles, the number of particles position- } \\
\text { ing in the } r \text { and } z \text { directions (NX and } N Y \text { ), } \\
\text { and the initial coordinates and space } \\
\text { increments must be specified on cards of } \\
\text { Type } 31 \text {. }\end{array}$ \\
\hline
\end{tabular}

Note: The fluid domains are identified by the marker-particle configuration; hence, particles must be used. 


\begin{tabular}{|c|c|c|c|c|}
\hline $\begin{array}{l}\text { Card } \\
\text { Type }\end{array}$ & Colums & Format & $\begin{array}{l}\text { FORTRAN } \\
\text { Name }\end{array}$ & Description \\
\hline \multicolumn{5}{|c|}{$\begin{array}{l}22 \\
(\text { contd.) }\end{array}$} \\
\hline & $1-12$ & & UINT & $\begin{array}{l}\text { Intial velocity in the } r \text { (or } x \text { ) direc- } \\
\text { tion, defined at the right-hand edge of } \\
\text { the cell, in } \mathrm{cm} / \mathrm{sec} \text {. }\end{array}$ \\
\hline & $13-24$ & & VINT & $\begin{array}{l}\text { Initial velocity in the } z \text { (or } y \text { ) direc- } \\
\text { tion, defined at the upper edge of the } \\
\text { cell, in } \mathrm{cm} / \mathrm{sec} \text {. }\end{array}$ \\
\hline & $25-36$ & & PINT & $\begin{array}{l}\text { Initial pressure, defined at the cell } \\
\text { center, in dynes } / \mathrm{cm}^{2} \text {. }\end{array}$ \\
\hline & $37-48$ & & EIINT & $\begin{array}{l}\text { Initial specific internal energy, de- } \\
\text { fined at the cell center, in } \mathrm{cm}^{2} / \mathrm{sec}^{2} \text {. }\end{array}$ \\
\hline & $49-60$ & & ETINT & $\begin{array}{l}\text { Initial specific total energy, defined } \\
\text { at the cell center, in } \mathrm{cm}^{2} / \mathrm{sec}^{2} \text {. }\end{array}$ \\
\hline
\end{tabular}

Note: Usually UINT, VINT, PINT, EIINT, and ETINT all equal zero.

23

$(5 E 12.4)$

$1-12$

$13-24$

$25-36$

$37-48$
AO

PRESS

ENERGY

GMA
Card for core-gas bubble and coolant.

$P_{0}$, initial pressure in the core-gas bubble, in dynes $/ \mathrm{cm}^{2}$.

$E_{0}$, initial total energy in the coregas bubble, in dyne-cm. (Energy > 0; if energy $=0$, the program will assume energy $=10^{12}$ dyne-cm.)

$a_{0}$, speed of sound of the fluid at standard condition, in $\mathrm{cm} / \mathrm{sec}$.

$n$, isentropic exponent of the fluid. It is a constant equivalent to the pressure derivative of bulk modulus of elesticity of the fluid. This constant appears in the Murnagham equation of state and will be used to calculate the $c_{1, j}^{n}$ through the relation: 


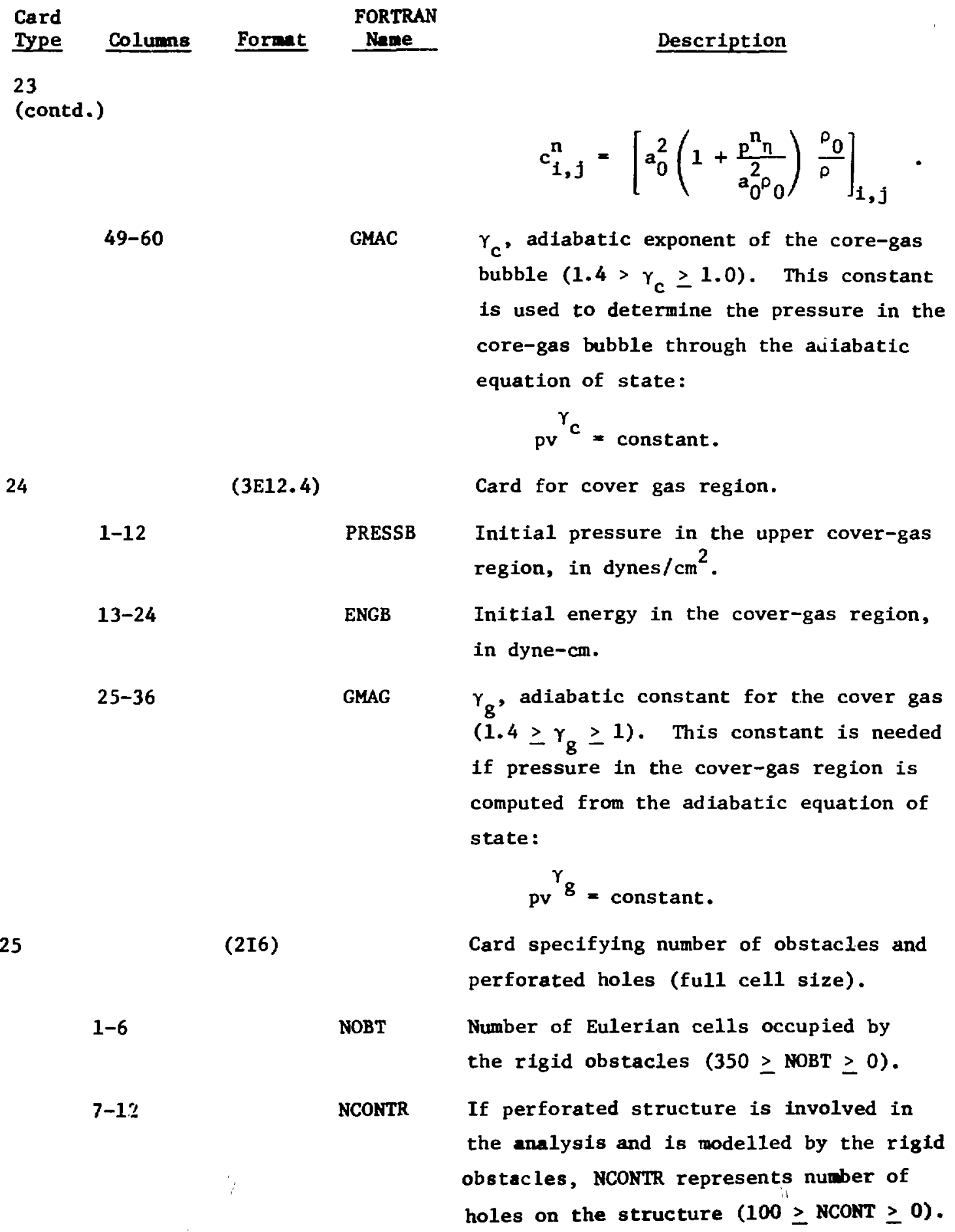

Initial energy in the cover-gas region, in dyne-cm.

GMAG

$\gamma_{g}$, adiabatic constant for the cover gas $\left(1.4 \geq r_{g} \geq 1\right)$. This constant is needed if pressure in the cover-gas region is computed from the adiabatic equation of state:

$$
{ }_{\text {pv }}^{\gamma} \mathbf{g}=\text { constant. }
$$

Card specifying number of obstacles and perforated holes (full cell size).

Number of Eulerian cells occupied by the rigid obstacles $(350 \geq$ NOBT $\geq 0)$.

If perforated structure is involved in the analysis and is modelled by the rigid obstacles, NCONTR represents number of holes on the structure $(100 \geq$ NCONT $\geq 0)$. 
Card FORTRAN

Type Colums Format Name Description

25

(contd.)

Note: (a) Card of Type 25 is needed when TYPE $=1.0$ specified on card 18.

(b) Specify the positions of the obstacle cells and cells occupied by the perforated holes, respectively, on cards of Type 26 and 27.

26

$$
\begin{aligned}
& \text { Obstacle location cards. } \\
& \left.\begin{array}{l}
\mathrm{KOBX}_{1} \\
\mathrm{KOBY}_{i}
\end{array}\right\} \\
& \mathrm{KOBX}_{i} \text { and } \mathrm{KOBY}_{i}(1=1,2, \ldots) \text { define, } \\
& \text { respectively, the radial and axial cell }
\end{aligned}
$$$$
\text { 1-6 }
$$$$
7-12
$$

Note: (a) There are NOBT (see card Type 25) sets of radial and axial cell numbers. Each card of Type 26 consists of 6 sets of them.

(b) Cards of Type 26 is required if Type $=1$ is specified on card of Type 18 .

27 Perforated-hole location cards.

1-6 7-12

$$
\left.\begin{array}{l}
\operatorname{KCONX}_{i} \\
\operatorname{KCONY}_{i}
\end{array}\right\}
$$
$\mathrm{KCONX}_{i}$ and $\mathrm{KCONY}_{i}(i=1,2, \ldots)$ define, respectively, the radial and axial cell number used to represent the position of the holes on the perforated plate.

Note: (a) There are NCONTR (see card Type 25) sets of radial and axial cell numbers. Each card of Type 27 consists of 6 sets of them.

(b) Cards of Type 27 is required only if TYPE $=1.0$ (specified on card of Type 18) and NCONTR $\neq 0$ (specified on card of Type 25).

Rigid thin membrane card.

$1-6$
IPLATE
Index indicating rigid thin boundary option.

IPLATE $=0$ : No rigid internal thin menbrane (or plate).

IPLATE = 1: With rigid thin membrane (or plate). 


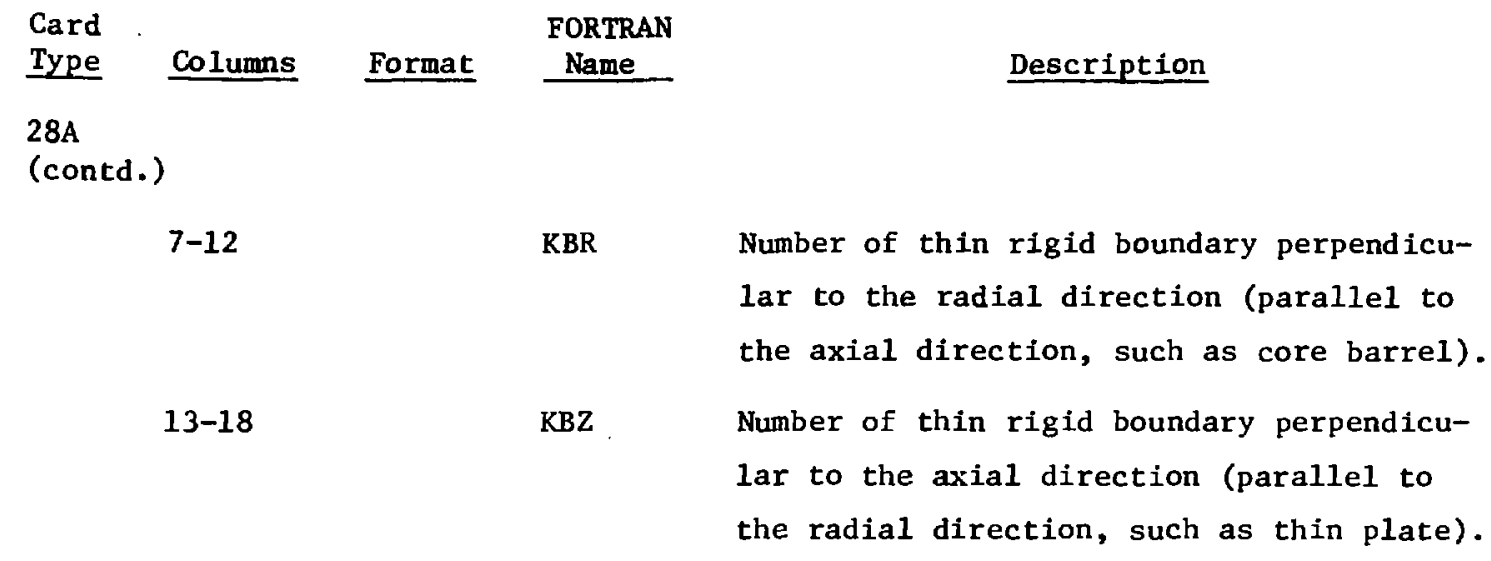

Note: (a) Rigid boundary option can be used to simulate shell or plate, either located internally or externally, but it must be located on the cell boundary.

(b) $\mathrm{KBZ} \neq 0$ only available if IZIP $=1$ is selected on card Type 5 .

28B

$\left.\begin{array}{ll}1-6 & \mathrm{IBR}_{i} \\ 7-12 & \mathrm{JBR}_{1}\end{array}\right\}$

Rigid thin shell location card.

$\mathrm{IBR}_{i}$ and $\mathrm{JBR}_{i}(i=1,2, \ldots, \mathrm{KBR})$ define, respectively, the radial and axial cell number of an Eulerian cell which has a rigid she11 locating at its right-hand cell boundary.

Note: (a) There are KBR (see card Type 28A) sets of radial axial cell number input. Each card Type 28B consists of 6 sets of them.

(b) Cards of Type $28 \mathrm{~B}$ are required if $\mathrm{KBR} \neq 0$ specified on card Type $28 A$.

$28 \mathrm{C}$

(1216)

$$
\begin{aligned}
& 1-6 \\
& 7-12 \\
& \text { etc. }
\end{aligned}
$$

Rigid thin plate location card.

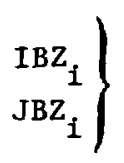
respectively, the radial and axial cell $\mathrm{IBZ}_{i}$ and $\mathrm{JBZ}_{i}(i=1,2, \ldots, \mathrm{KBZ})$ define, number of an Eulerian cell which has a rigid thin plate locating at its upper ce11 boundary. 


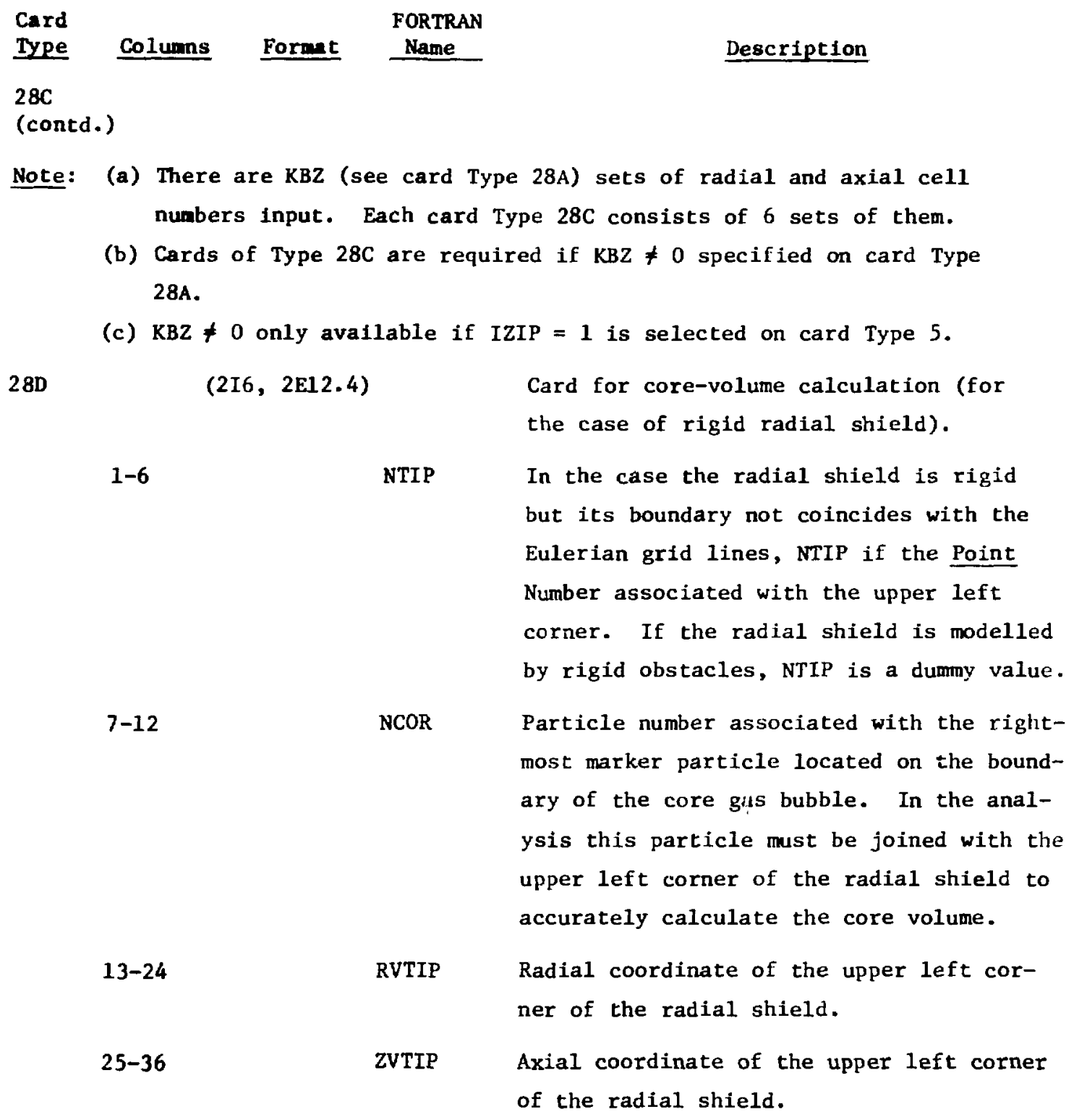

Note: Card of Type 28D is required if IEXPN $=1$ specified on card of Type 7 . shield). which define the upper left corner of the flexible radial shield. 


\begin{tabular}{|c|c|c|c|c|}
\hline $\begin{array}{l}\text { Card } \\
\text { Type }\end{array}$ & Columns & Format & $\begin{array}{l}\text { FORTRAN } \\
\text { Name } \\
\end{array}$ & Description \\
\hline \multicolumn{5}{|c|}{$\begin{array}{l}28 E \\
\text { (contd.) }\end{array}$} \\
\hline & $7-12$ & & NCOR & $\begin{array}{l}\text { Same description as NCOR on card of Type } \\
\text { 28D. }\end{array}$ \\
\hline Note: & Card of : & $\begin{array}{r}\text { Type } 28 \mathrm{E} \text { is } \\
(6 \mathrm{E} 12.4)\end{array}$ & required if & $\begin{array}{l}\text { IEXPN = } 2 \text { specified on card of Type } 7 \text {. } \\
\text { Cards provide non-zero initial values } \\
\text { for the plotting curves. }\end{array}$ \\
\hline & $\begin{array}{l}1-12 \\
13-24 \\
\cdot \\
\cdot \\
67-72\end{array}$ & & $\begin{array}{l}\operatorname{PRS}_{1,1} \\
\operatorname{PRS}_{2,1} \\
\cdot \\
\cdot \\
\operatorname{PRS}_{\mathbf{m}, 1}\end{array}$ & $\begin{array}{l}\text { PRS }_{\mathrm{m}, 1} \text { is the initial value associated } \\
\text { with each } I_{m} \text { and } J_{m} \text { defined in cards of } \\
\text { Type } 4 . \text { These values should be speci- } \\
\text { fied in the same order as on the cards } \\
\text { of Type } 4 \text { and will be printed in the } \\
\text { output. }\end{array}$ \\
\hline
\end{tabular}

Note: (a) Cards of Type 29 are not needed if NPP $=0$ or in NPRS $=0$ is specified on card of Type 3.

(b) There are NPP (see card Type 3) initial values. Each card Type 29 consists of 6 of them.

Nonuniform initial condition card (Case 1: NP $\geq 0$ on card of Type 6).

$1-6$

$7-12$

13-24

25-36

$37-48$

49-60

61-72
$\mathrm{U}_{\mathrm{I}, \mathrm{J}}$

$$
\mathrm{V}_{\mathrm{I}, \mathrm{J}}
$$

$$
P_{i, j}
$$$$
E_{1, j}
$$$$
\mathrm{RHO}_{1, j}
$$

Cell number of the $r$ (or $x$ ) direction.

Cell number in the $z$ (or $y$ ) direction.

Velocity in the $r$ (or $x$ ) direction, defined at right edge of the cell, in $\mathrm{cm} / \mathrm{sec}$.

Velocity in $z$ (or $y$ ) direction, defined at the top edge of the cell, in $\mathrm{cm} / \mathrm{sec}$.

Pressure at the cell center, in dynes $/ \mathrm{cm}^{2}$.

Specific internal energy, defined at the cell center, in $\mathrm{cm}^{2} / \mathrm{sec}^{2}$.

Density at the cell center, in $8 \mathrm{~m} / \mathrm{cm}^{3}$. 


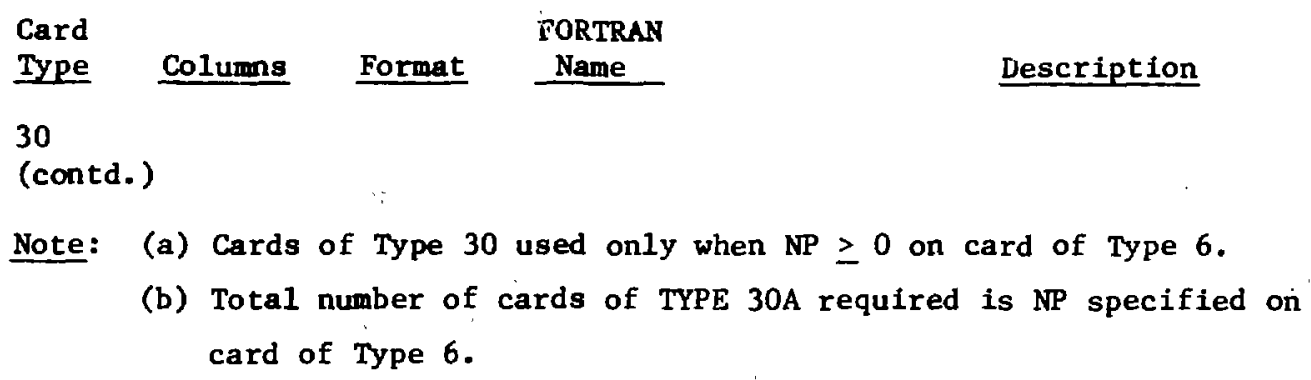

Note: (a) Cards of Type 30 used only when NP $\geq 0$ on card of Type 6 .

(b) Total number of cards of TYPE 30A required is NP specified on card of Type 6 .

$30 \mathrm{~A}$

(a1ternative)

$1-3$

4-6 I2

$7-9$

$10-12$

$30 \mathrm{~B}$

(alternative)

$13-24$
$25-36$
$37-48$
$49-60$
$61-72$

$\mathbf{U}_{I, J}$

$v_{i, j}$

$P_{1, j}$

See card of Type $30 \mathrm{~A}$ for descriptions.

$\mathrm{RHO}_{\mathbf{1}, \mathrm{j}}$

Nonuniform initial condition card (Case 2: NP $<0$ on card of Type 6). $\mid$ NP $\mid$ define the number of sections for specifying the initial conditions.

Starting cell number in the $r$ (or $x$ ) direction.

End cell number in the $r$ (or $x$ ) direction.

Starting cell number in the $z$ (or $y$ ) direction.

Ending cell number in the $z$ (or $y$ ) direction.

Note: (a) Cards of Type 30B used only when NP $<0$ specified on card of Type 6.

(b) Total number of cards of Type 30B is $|\mathrm{NP}|$ specified on cards of Type 6.

(c) Here U, V, P, EI, RHO are the uniform condition throughout a region bounded by $\mathrm{I} 1, \mathrm{I} 2, \mathrm{~J} 1$, and $\mathrm{J} 2$. 


\begin{tabular}{|c|c|c|c|c|}
\hline $\begin{array}{l}\text { Card } \\
\text { Type }\end{array}$ & Column: & Format & $\begin{array}{l}\text { FORTRAN } \\
\text { Nene } \\
\end{array}$ & Description \\
\hline 31 & \multicolumn{3}{|c|}{$(2 \mathrm{I} 6,4 \mathrm{E} 12.4)$} & Particles set-up cards. \\
\hline & $1-6$ & & NX & $\begin{array}{l}\text { Number of columns of marker particles to } \\
\text { be created in the } r \text { (or } x \text { ) direction. }\end{array}$ \\
\hline & $7-12$ & & NY & $\begin{array}{l}\text { Number of rows of marker particles to be } \\
\text { created in the } z \text { (or } y \text { ) direction. }\end{array}$ \\
\hline & $13-24$ & & Xo & $\begin{array}{l}r(o r x) \text { coordinate of first particle, } \\
\text { in } \mathrm{cm} .\end{array}$ \\
\hline & $25-36$ & & YO & $\begin{array}{l}z \text { (or y) coordinate of first particle, } \\
\text { in } \mathrm{cm} \text {. }\end{array}$ \\
\hline & $37-48$ & & DXK & $\begin{array}{l}\text { Particle spacing in the } r \text { (or } x \text { ) direc- } \\
\text { tion, in } \mathrm{cm} \text {; negative value representing } \\
\text { decrement. }\end{array}$ \\
\hline & $49-60$ & $i$ & DYK & $\begin{array}{l}\text { Particle spacing in the } z \text { (or } y \text { ) direc- } \\
\text { tion, in cm; negative value representing } \\
\text { decrement. }\end{array}$ \\
\hline
\end{tabular}

Note: (a) Card of Type 31 is needed for each set of particles. Thus the total number of cards of this type is equal to the value of $M$ specified on card of Type 21.

(b) Particle sets indicating the initial position of bubble boundary must be specified first and should be numbered in the clockwise direction. The total number of particles used is equal to MPA defined on card of Type 7.

(c) Particle set defining the initial position of the free surface should be input next and should be numbered in the direction of decreasing $r$. The total number used is equal to MPB defined on card of Type 7 . arbitrarily shaped external boundaries $(50 \geq$ NIRG $\geq 0)$. 


Card
Type Columns Format $\quad \begin{aligned} & \text { FORTRAN } \\ & \text { Name }\end{aligned} \quad$ Description

32

(contd.)

Note: Cards of Type 39 is needed cnly if IVESSL $=3$ specified on card of Type 6. Here the external toundaries can be of arbitrarily shape but are rigidly fixed in the space.

33

$(4 \mathrm{E} 12.4)$
$1-12$
$\left.\begin{array}{c}\mathrm{RB}_{1} \\ \mathrm{ZB}_{1}\end{array}\right\}$
$13-24$
$25-36$
$37-48$

$$
\left.\begin{array}{c}
\mathrm{RT}_{i} \\
\mathrm{ZT}_{i}
\end{array}\right\}
$$
$R B_{i}$ and $Z B_{i}$ define, respectively, the radial and axial coordinates of the beginning node point associated with

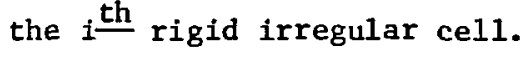 $\mathrm{RT}_{1}$ and $\mathrm{ZT}_{i}$ define, respectively, the radial and axial coordinates of the terminating node point associated with the $i$ th igid irregular cell.

Rigid irregular cell cards.

Note: (a) The number of cards of Type 33 required is NIRG specified on card of Type 32.

(b) This card is omitted if IVESSL $\neq 3$ on card of Type 6.

34

1-6

$7-12$

ISTR
Card for hydrodynamics of the deformable internals.

Total number of load lines (Lagrangian boundary lines) along which the sliding boundary conditions are satisfied. Specify the beginning node point and terminating point of each load lines on cards of Type 35.

Index indicating nature of the internal structure.

ISTR $=0$ : Load Iines do not enclose thick component which completely occupies one or more Eulerian cell.

ISTR > 1: Load lines may enclose thick component which occupies one or more 
Card

Type
FORTRAN

Columns Format Name

34

(contd.)
$13-18$

$19-24$

$25-30$

$31-36$

\begin{tabular}{|c|c|c|}
\hline $13-18$ & NSEG & $\begin{array}{l}\text { Number of segment at which sliding } \\
\text { boundary condition is not required. } \\
\text { This situation occurs when structural } \\
\text { segment does not contact with the fluid. } \\
\text { If NSEG > } 0 \text { specify these segments on } \\
\text { cards of Type } 37 \text {. }\end{array}$ \\
\hline $19-24$ & $\mathrm{NCON}$ & $\begin{array}{l}\text { Number of straight lines to be connected } \\
\text { in the film output (marker particle con- } \\
\text { figuration plot) for convenience of in- } \\
\text { dicating the internal component. If } \\
\text { NCON > } 0 \text { specify the beginning and ter- } \\
\text { minating points of each straight lines } \\
\text { on card of Type } 38 \text {. Note that these } \\
\text { points must be located on the load lines. }\end{array}$ \\
\hline $25-30$ & NPZERO & $\begin{array}{l}\text { Number of segments not subjected to the } \\
\text { hydrodynamic pressure. This situation } \\
\text { occurs if the structure segment does } \\
\text { not contact with the fluid. If NPzERO } \\
>0 \text { specify segment numbers on card of } \\
\text { Type } 39 \text {. }\end{array}$ \\
\hline $31-36$ & NOPEN & $\begin{array}{l}\text { Number of pair segments (one top and one } \\
\text { bottom) on the core-support structure } \\
\text { having perforated openings. If NOPEN >0 } \\
\text { specify segment number, perforated ratio, } \\
\text { and other information on card of Type } 40 \text {. }\end{array}$ \\
\hline
\end{tabular}

Eulerian cell. In this case, specify the beginning and terminating node points of those load lines (either entire or part of the line) on card of Type 36 .

Number of segment at which sliding segment does not contact with the fluid. If NSEG > 0 specify these segments on in the film output (marker particle configuration plot) for convenience of inminating points of each straight lines on card of Type 38 . Note that these 


Card
Type Columns Format $\begin{aligned} & \text { FORTRAN } \\ & \text { Name }\end{aligned}$ Description

34

(contd.)

$37-42$

43-48
NBLDIS

ILOAD

\author{
Index indicating geometrical discontin- \\ uities generated by the deformable core \\ barrel. \\ NBLDIS $=0$ : No core-barre1. \\ NBLDIS $=1$ : With core-barrel discontinuity. \\ Provide input on cards of Type 41. \\ Index for determining load line type. \\ ILOAD = 0: Load line type (LTLOAD) deter- \\ mined internally. \\ ILOAD = 1: Load line type prescribed by \\ the user. Provide input on card 34A.
}

Note: (a) If ILOAD $=0$ types of all the load lines are determined by the value of IVESSL on card 6 ; that is IVESSL < 4, $\operatorname{LTLAD}_{i}=1$ (external structure); for IVESSL $=4, \operatorname{LTLOAD}_{i}=2$ (internal structure).

49-54 KFLD

$34 \mathrm{~A}$

(12I6)

1-6

$7-12$
Index for prescribing the boundary flags. KFLD $=0:$ Boundary flag determined internally.

KFLD $=1:$ Boundary flag of certain load line is prescribed by the user. Provide input on card $34 \mathrm{~B}$.

Load line type.

Integer indicating load line type.

LTLOAD = 1: External structure.

LTLOAD = 2: Internal structure.

Note: (a) Cards of Type 34A is required only if ILOAD $\neq 0$ on card 34 .

(b) Use as many cards of Type $34 \mathrm{~A}$ as required; $i=1,2, \ldots$, NLOAD.

(c) $L T L O A D=2$, multi-value field variables are used in the analysis. 
Card

Type

34B

$\begin{array}{lll}\text { Columns } & \text { Format } & \begin{array}{l}\text { FORTRAN } \\ \text { Name }\end{array} \\$\cline { 1 - 2 } & \text {$\left.(12I6) } & \\ 1-6 & & \text { IDEXLD }_{i} \\ 7-12 & & \text { KFLOAD }_{i}\end{array}\right\}$

\section{Description}

Boundary flag of load line.

$\operatorname{IDEXLD}_{i}$ and $\mathrm{KFLOAD}_{i}$ provide index and

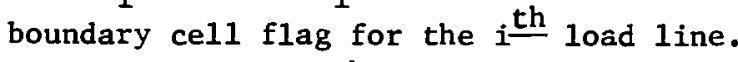

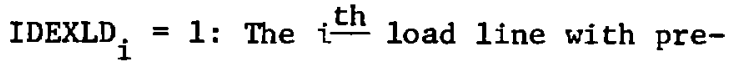
scribed boundary flag.

$\mathrm{KFLOAD}_{i}: \mathrm{A}$ numeral indicating orientation of the boundary segment.

Note: (a) Cards of Type 34B is required only if KFLD $\neq 0$ on card 34 .

(b) There are NLOAD (see card Type 34) sets of index and boundaryflag input. Each card consists of 4 sets of them.

(c) See Section $V$ for description of the boundary cell flag.

35

$\left.\begin{array}{ll}1-6 & \text { NBEG }_{i} \\ 7-12 & \text { NTER }_{i}\end{array}\right\}$

Load line cards.

$\mathrm{NBEG}_{i}$ and $\operatorname{NTER}_{i}(i=1, \ldots$ NLOAD) define, respectively, the beginning and terminating point numbers for the $i$ th load line.

Note: There are NLOAD (see card 34) sets of input. Each card consists of 6 of them.

36

(12I6)

$\left.\begin{array}{ll}1-6 & \text { NSTRB }_{i} \\ 7-12 & \text { NSTRT }_{i}\end{array}\right\}$

$\cdot$

$\cdot$

$\cdot$
Thick structure card.

$\mathrm{NSTRB}_{i}$ and $\mathrm{NSTRT}_{i}$ define, respectively, the two points (not necessary to be the beginning and terminating points) on the load lines which may enclose component having thickness more than one Eulerian cell.

Note: There are ISTR (see card Type 34) sets of input. Each card consists of 6 of them.

1-6

7-12

etc.
KSEG $_{\mathbf{i}}$

Segment number where sliding boundary condition at the midpoint of that segment is not required. 
Card
Type Columns Format $\quad \begin{gathered}\text { FORTRAN } \\ \text { Name }\end{gathered} \quad$ Description

37

(contd.)

Note: Use as many cards of Type 37 as required; 12 numbers per each card $(1=1,2, \ldots, N S E G)$.

38

(12I6)

$\left.\begin{array}{ll}1-6 & \mathrm{NCONB}_{i} \\ 7-12 & \mathrm{NCONT}_{i}\end{array}\right\}$

$\mathrm{NCONB}_{i}$ and $\mathrm{NCONT}_{i}(i=1,2, \ldots, \mathrm{NCON})$

etc. define, respectively the beginning and terminating points on the $i$ th load line to be connected in the film output. The cross hatch line used to indicate the radial shield.

Note: There are NCON (see card Type 34) sets of beginning and terminating points. Each card of Type 38 consists of 6 of them.

39

(12I6)

\begin{tabular}{|c|c|c|}
\hline $1-6$ & KPZERO $_{i}$ & Some structural segments do not contact \\
\hline $7-12$ & & with the fluid such as if the segments \\
\hline - & & are the internal members of the structure. \\
\hline • & - & $\begin{array}{l}\text { In this case, } \mathrm{KPZERO}_{i}(i=1,2, \ldots, \\
\text { NPZERO }) \text { defines the numbers of those seg- } \\
\text { ments. }\end{array}$ \\
\hline
\end{tabular}

Note: Use as many cards of Type 39 as required; $i=1, \ldots$, NPZERO.

40

\begin{tabular}{|c|c|c|}
\hline \multicolumn{2}{|c|}{$(2 I 6,5 E 12.4)$} & Perforated core support structure. \\
\hline $1-6$ & NOSEG1 $_{i}$ & $\mathrm{~N} 1_{i}$ and $\mathrm{N} 2_{i}(i=1,2, \ldots$ NOPEN $)$ define, \\
\hline $7-12$ & NOSEG $_{i}$ & $\begin{array}{l}\text { respectively, the bottom and the top seg- } \\
\text { ment numbers of the core-support structure. }\end{array}$ \\
\hline $13-24$ & RATIO $_{i}$ & $\begin{array}{l}\phi_{i} \text {, perforated ratio (ratio of opening } \\
\text { area to the total area of the segment). }\end{array}$ \\
\hline $25-36$ & $\mathrm{UOPEN}_{i}$ & $\begin{array}{l}U_{i} \text {, initial velocity in the openings, in } \\
\mathrm{cm} / \mathrm{sec} \text { (normally zero). }\end{array}$ \\
\hline
\end{tabular}




\begin{tabular}{|c|c|c|c|c|}
\hline $\begin{array}{l}\text { Card } \\
\text { Type }\end{array}$ & Columns & Fornat & $\begin{array}{c}\text { FORTRAN } \\
\text { Name } \\
\end{array}$ & Description \\
\hline \multicolumn{5}{|c|}{$\begin{array}{l}40 \\
(\text { contd.) }\end{array}$} \\
\hline & $37-48$ & & HIGHT $_{1}$ & $\begin{array}{l}\mathrm{L}_{i} \text {, thickness or length of the opening } \\
\text { passage, in } \mathrm{cm} \text {. }\end{array}$ \\
\hline & $49-60$ & & $\operatorname{COEF}_{i}$ & $\begin{array}{l}\text { Frictional loss coefficient, used to calcu- } \\
\text { late the pressure drop in the coolant passages. }\end{array}$ \\
\hline & $61-72$ & & EXPONT $_{i}$ & Exponential factor. \\
\hline
\end{tabular}

Note: (a) Cards of Type 40 are required if NOPEN > 0 specified on cards of Type 34 .

(b) Total number of cards of type required is NOPEN.

41

$1-6$

$7-12$
(2I6)

NPTR Point number define the upper right end of the flexible core barrel.

NPTL Point number define the upper left enid of the flexible core barrel.

Note: Card of Type 41 is used if NBLDIS $\neq 0$ specified on card of Type 34 .

42

1-6

IOPEN

$7-12$
Movable cover and sodium spillage.

Radial cell number where sodium spillage through vessel-head junction or plug jump occurs. For rigid vessel, IOPEN = IBAR; for the flexible vessel, IOPEN $=I_{r}+1$ in which $I_{r}$ is the cell number of the rightmost quasi-Eulerian (irregular) cell adjacent to the ressel cell.

Axial cell number where sodium spillage through vessel-head junction or plug jump occurs. For the rigid vessel or flexible vessel, JOPEN $=J_{Z}+1$, in which $\mathrm{J}_{Z}$ is the axial cell number of the uppermost quasi-Eulerian (irregular) cell below the vessel head. Note that to allow 


Card
Type Colums Format $\quad \begin{gathered}\text { FORTRAN } \\ \text { Name }\end{gathered}$

42

(contd.)

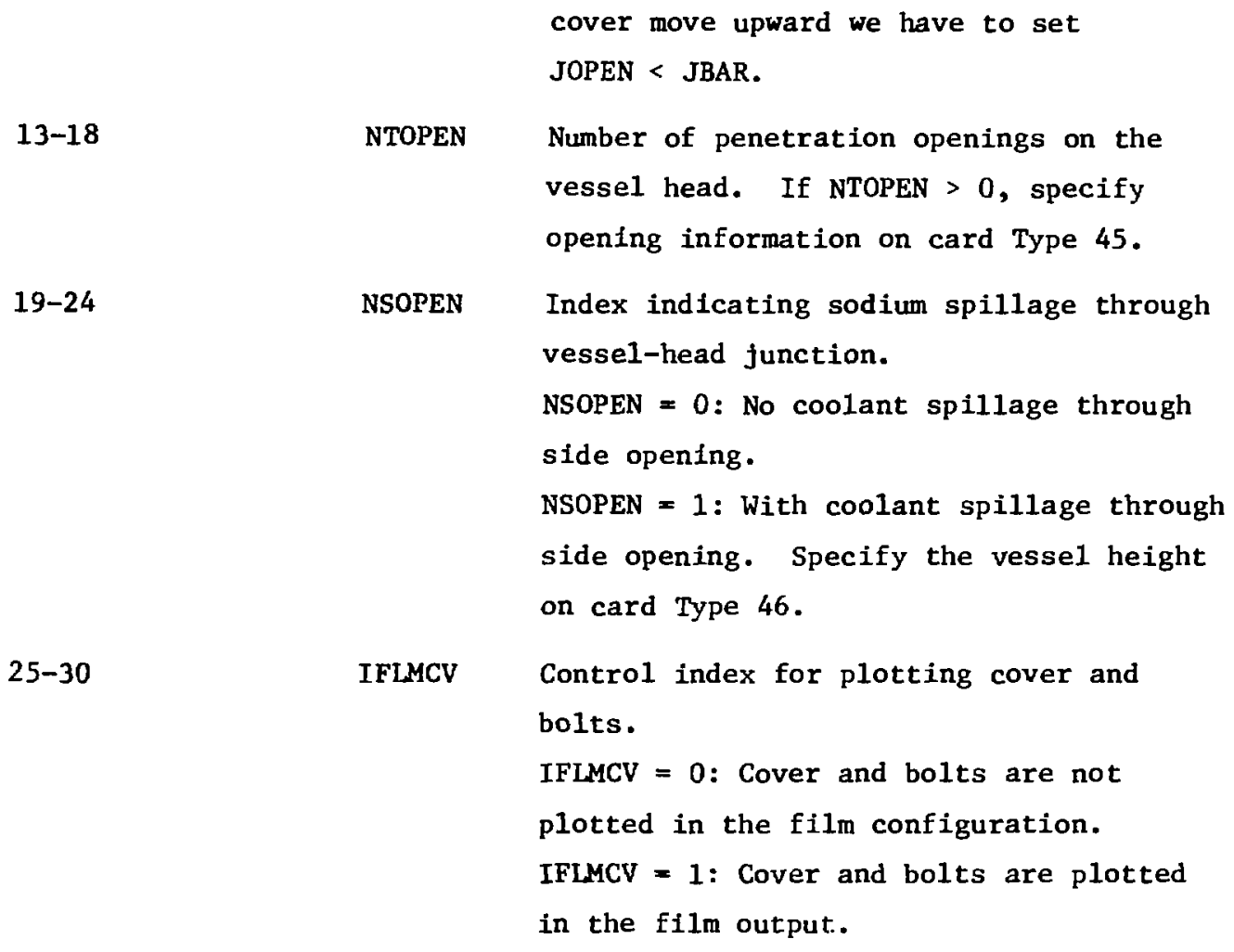

Note: (a) Card Type 42 is required when either INDOUT $>1$ or ICOVER $\neq 0$ specified on card Type 6.

(b) When sodium spillage is considered (INDOUT $>1$ ), reactor cover must be considered as movable (ICOVER $>0$ ) even if rigid cover is used. Thus, cards of Type 43 and 44 should be included.

$$
(2 I 6,3 E 12.4)
$$

1-6

7-12
Movable reactor cover.

Number of points on the force-displacement table for describing the response of the cover and bolts.

Index indicating relative position of the free surface and the cover head.

ICONT = 0: Coolant not contacting with cover head initially. 


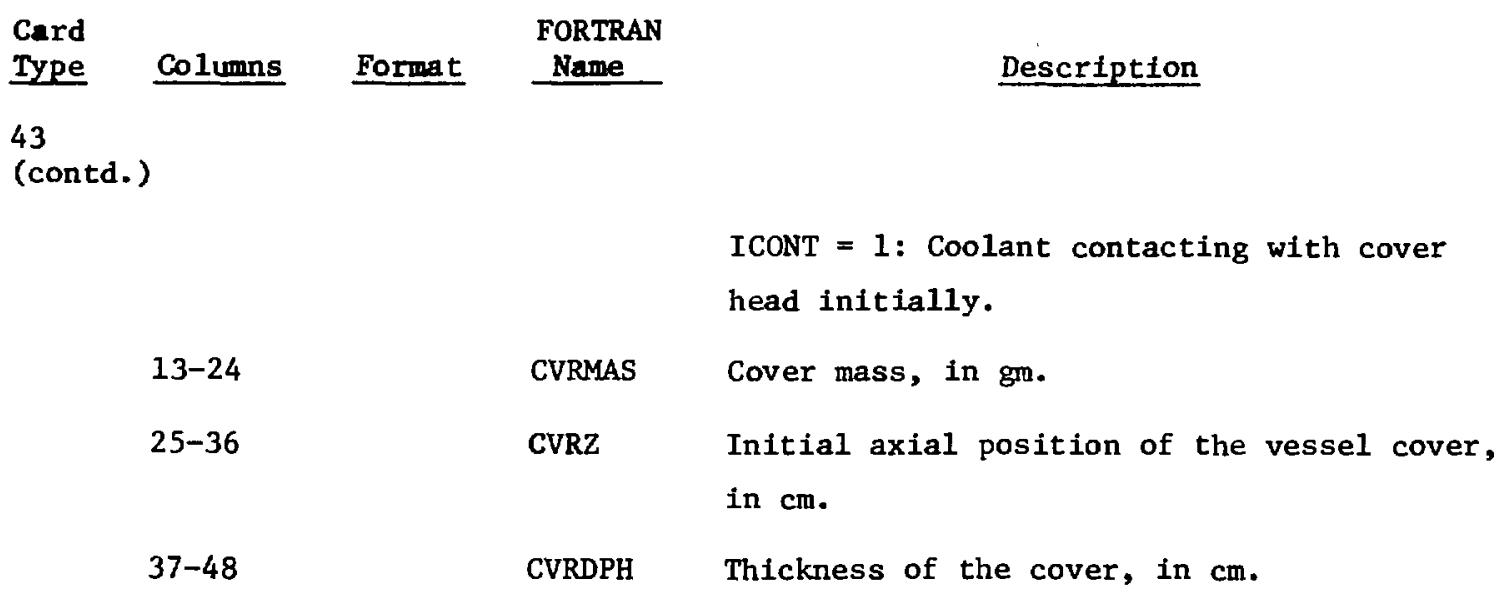

Note: Cards of Type 43 is required when either INDOUT $>1$ or ICOVER $\neq 0$ specified on card Type 6.

44

$$
\text { (I6, 2E12.4) }
$$

Bolts card.

$1-6$

K

Point number.

$7-18$

$\mathrm{CF}_{\mathrm{K}}$

Force, in dynes $/ \mathrm{cm}^{2}$.

19-30

$\mathrm{CD}_{\mathrm{K}}$

Displacement, in $\mathrm{cm}$.

Note: (a) Cards of Type 44 are required if either INDOUT $>1$ (ICOVER $>0$ ) or ICOVER $\neq 0$ specified on card Type 6 .

(b) Total number of card Type 44 required is KCVR specified on card Type 43.

(c) The displacements on card Type 44 must be in increasing order.

(d) To simulate the rigid cover one can specify large boit force but small displacement.

45

$(2 \mathrm{I} 6,2 \mathrm{E} 12.4)$

$1-6$

$7-12$

13-18

19-24
$\mathrm{KNN}_{\mathrm{K}}$ $\mathrm{FNTP}_{\mathrm{K}}$

FNTAR
Penetration openings card.

Radial cell number with penetration opening.

Flag for $k^{\text {th }}$ radial cell. KNN $=1$ : With penetration.

Allowable impulse of penetration above which the penetration will become open, in dyne-sec/ $/ \mathrm{cm}^{2}$.

opening ratio. 


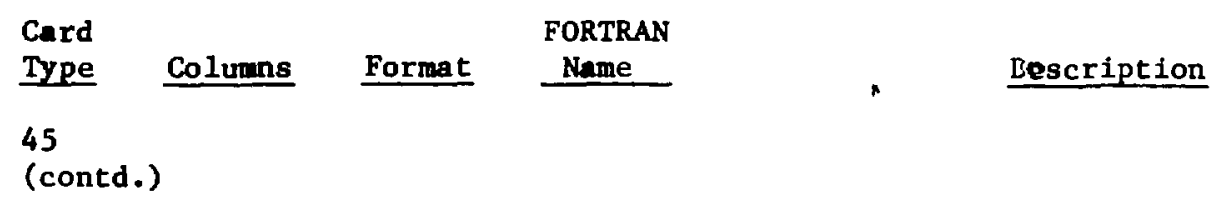

Note: (a) Cards of Type 45 is required only if INDOUT $>1$ on card of Type 6 .

(b) Total number of card Type 45 required is NTOPEN specified on card Type 42.

(E12.4)

$1-12$

HTMAX

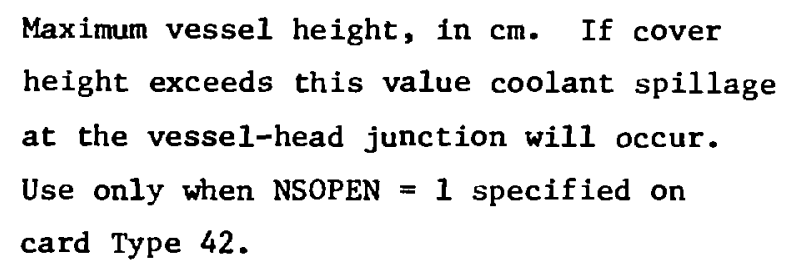

Note: Cards of Type 47 and 48 are not used. They are reserved for problems involving multi-material interaction.

49

$1-6$

$7-12$

ICSS

$13-18$

NPERF

19-24

IUIS
Parameter card.

Index for indicating the characteristic of the Upper Internal Structure (UIS).

IUIS $=0$ : No rigid, perforated UIS.

IUIS $=1:$ With rigid, perforated UIS, input the radial and axial cell numbers for each perforated obstacle.

Index for indicating the characteristic of the Core-Support Struzture (CSS).

ICSS $=0$ : No rigid and perforated CsS. ICSS = 1: With rigid and perforated CSS, input the radial and axial cell numbers for each perforated obstacle.

Total number of perforated-obstacle cells.

INDUIS
INDUIS $=1:$ Control-volume method for analyzing flows through the UIS. INDUIS $\neq 1$ : Other methods (not available). 
Card
Type Colunns Format

49

(contd.)

25-30

$31-36$

$37-42$

$43-48$

49-54

$55-60$

50

$1-6$

(4I6)

$7-12$

1.3-18

INDCSS

JREF

IDPUIS
INDCSS $=1$ : Control-volume method for analyz-

ing flows through the CSS.

INDCSS $\neq 1$ : Other methods (not available).

Axial cell number separating the UIS and the

css,

$J R E F-1<J_{B}$, where $J_{B}$ is the axial cell number below the perforated UIS.

MOPEN Number of columns of cells representing the perforated UIS.

NOPEN Number of columns of cells representing the perforated CSS.

NDGPEF Frequency of printout for flow through the perforated structure.

ITUBF, ITUBE $=1$ : Perforated UIS remains intact, only axial fluid motion is involved.

ITUBE $\neq 1$ : Not available.

Parameter card.

Index for pressure-loss calculation for the UIS.

IDPUIS $=0$ : Pressure loss is not calculated. IDPUIS = $1:$ Pressure loss is calculated (not available).

IDPCSS Index for pressure-loss calculation for the cSs.

IDPCSS $=0$ : Pressure loss is not calculated. IDPCSS $=1:$ Pressure loss is calculated

(not available).

IMVUIS IMVUIS $=0$ : Particles move uniformly through the perforated UIS.

IMVUIS \& 0: Not available. 
\begin{tabular}{l} 
Card \\
Type Columns Format $\begin{array}{c}\text { FORTRAN } \\
\text { Name }\end{array}$ \\
\hline
\end{tabular}

50

(contd.)

(12I6)

$1-6$

$7-12$

•

IMVCSS

IMVCSS $=0:$ Particles move uniformly through the perforated CSS.

IMVCSS $\neq 0$ : Not available.

Description

Perforated-structure location cards.

IPERF $_{i}$ IPERF $_{i}$ and JPERF $_{i}(i=1,2 \ldots$ NPERF $)$ define, JPERF $_{i} \quad$ respectively, the radial and axial cell number for representing the perforated UIS and CSS.

Note: (a) There are NPERF (see card Type 49) sets of radial and axial cell numbers. Each card of Type 51 consists of 6 sets of them.

(b) Card of Type 51 is required of NPERF $\neq 0$ is specified on card Type 49. (UIS).

$1-6$

$\mathrm{K}$

Radial cell number.

$7-18$

$19-30$

RATUIS $_{k}$ Perforation ratio for cells having radial cell number $k$.

DIAUIS $_{\mathbf{k}}$

Equivalent hydraulic diameter (in $\mathrm{cm}$ ) for calculating the frictional loss in the UIS (not available).

$31-42$ RGHUIS $_{k}$ Coeffictent for indicating roughness corresponding to cells with radial cell number $k$ (not available)

Note: (a) Cards of Type 52 are required if IUIS $=1$ and INDUIS $=1$ specified on card Type 49.

(b) Total number of card Type 52 required is MOPEN specified on card Type 49. 


\begin{tabular}{|c|c|c|c|c|}
\hline $\begin{array}{l}\text { Card } \\
\text { Type }\end{array}$ & Columns & Format & $\begin{array}{l}\text { FORTRAN } \\
\text { Name }\end{array}$ & Description \\
\hline \multicolumn{5}{|c|}{$\begin{array}{l}53 \\
\text { (contd.) }\end{array}$} \\
\hline & $1-6$ & & $\mathbf{K}$ & Radial cell number. \\
\hline & $7-18$ & & $\operatorname{RATCSS}_{k}$ & $\begin{array}{l}\text { Perforation ratio for cells having radial } \\
\text { cell number } k \text {. }\end{array}$ \\
\hline & $19-30$ & & DIACSS $_{k}$ & $\begin{array}{l}\text { Equivalent hydrodynamic diameter (in } \mathrm{cm} \text { ) } \\
\text { for calculating the frictional loss in the } \\
\text { CSS (not available). }\end{array}$ \\
\hline & $31-42$ & & RGHCSS $_{k}$ & $\begin{array}{l}\text { Coefficient for indicating roughness corre- } \\
\text { sponding to cells with radial cell number } k \\
\text { (not available). }\end{array}$ \\
\hline
\end{tabular}

Note: (a) Cards of Type 53 are required if ICSS $=1$ and INDCSS $=1$ specified on card Type 49.

(b) Total number of card Type 53 required is NOPEN specified on card Type 49. 


\section{B, Finite-Element Structural Dynamics Input}

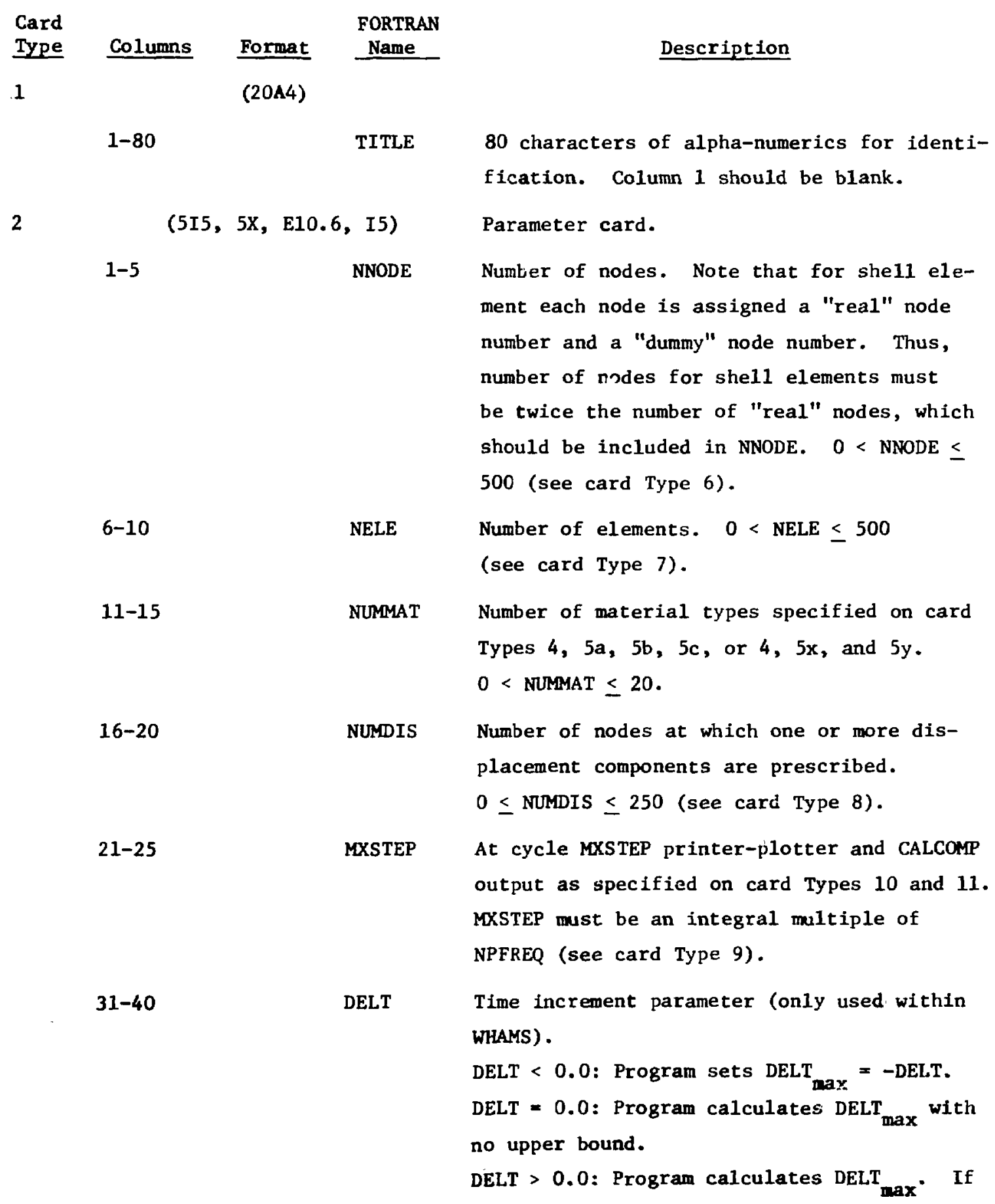




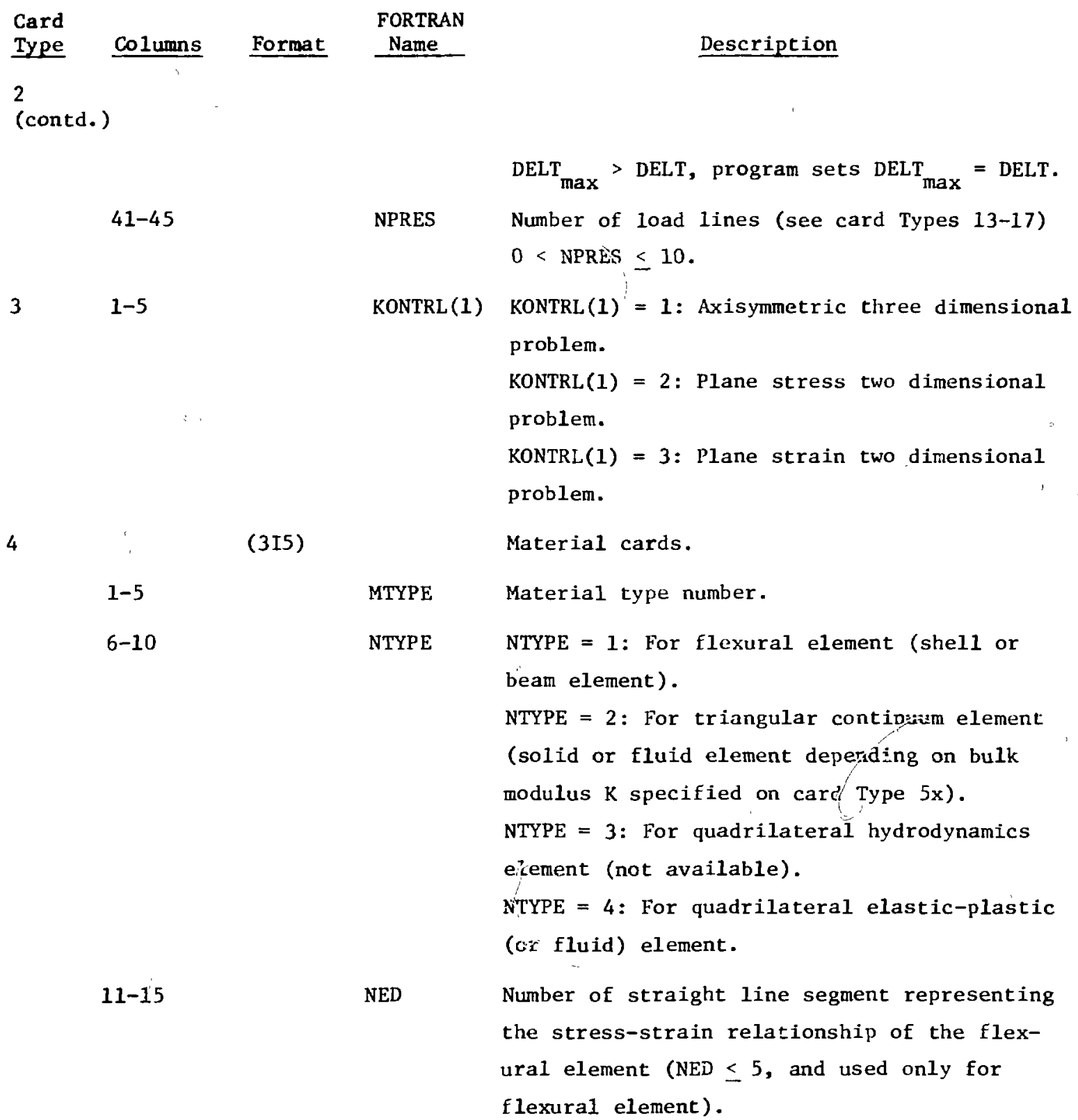

Note: (a) The flexural element (NTYPE $=1$ ) is used to model the reactor vessel, core barrel, and core-support structure.

(b) The quadrilateral elastic-plastic element (NTYPE $=4$ ) is suggested to model the radial shield or other heavy fluids. 


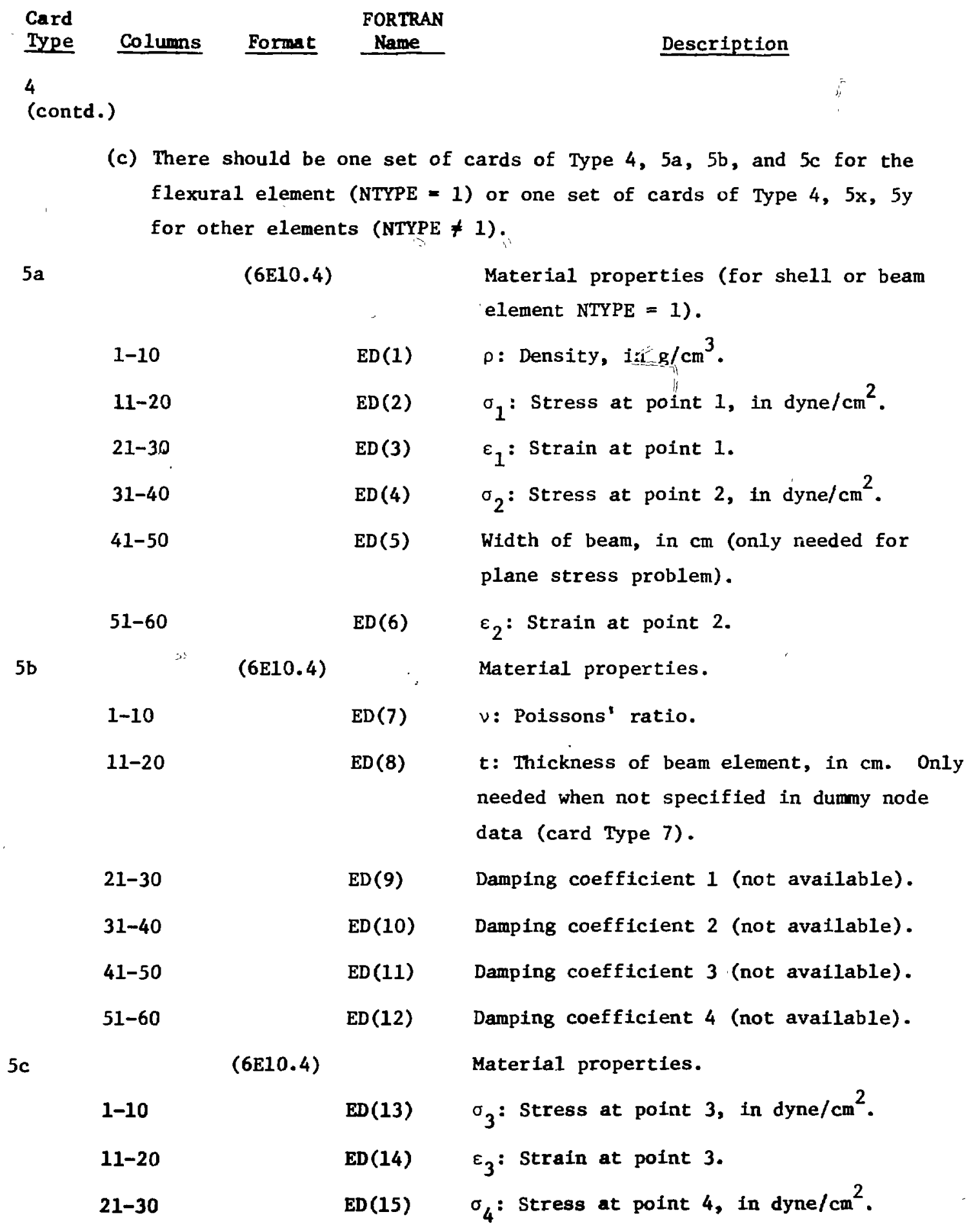




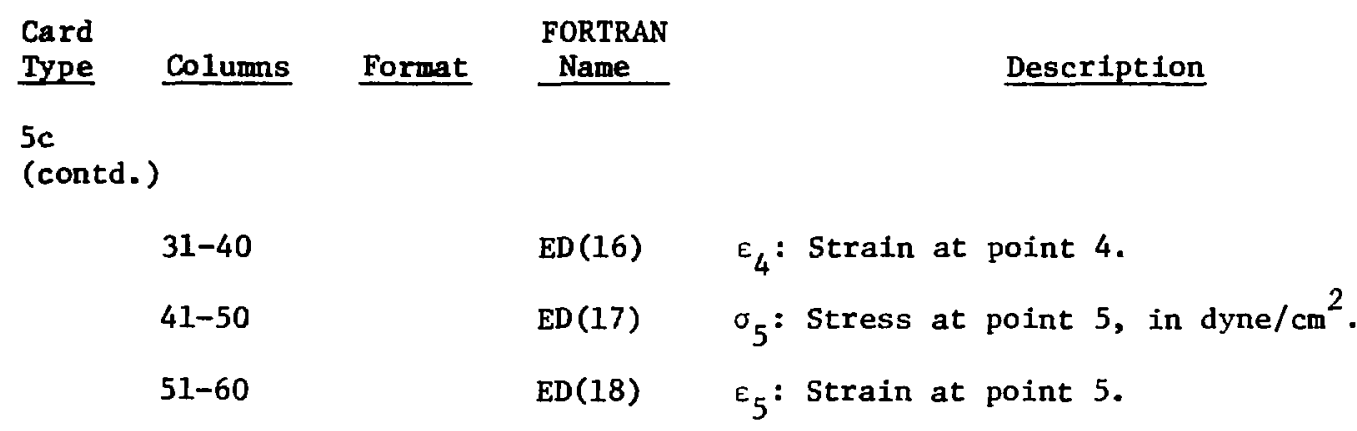

Note: In the case of NED $\leq 2$ specified on card Type 4, card of Type 5c is simply a blank card.

$5 \mathrm{x}$

$(6 \mathrm{E} 10.4)$

Material properties (for continuum elements NTYPE $\neq 1$ ).

$$
\begin{array}{lll}
1-10 & E(1, \text { MTYPE }) & \rho: \text { Density, in } \mathrm{g} / \mathrm{cm}^{3} . \\
11-20 & E(2, \text { MTYPE }) & \text { E: Young's modulus, in dyne } / \mathrm{cm}^{2} . \\
21-30 & E(3, \text { MTYPE }) & \sigma_{y}: \text { Initial yield stress, in dyne } / \mathrm{cm}^{2} . \\
31-40 & E(4, \text { MTYPE) } & \text { Ep: Plastic modulus, in dyne } / \mathrm{cm}^{2} . \\
41-50 & E(5, \text { MTYPE) } & \text { B }_{0}: \text { Bulk modulus of elasticity, in dyne } / \mathrm{cm}^{2} \\
& \text { (for NTYPE }=3 \text {, or } 4) . \\
51-60 & \text { E(6,MTYPE) } & \sigma_{u l t}: \text { Ultimate stress, in dyne } / \mathrm{cm}^{2} .
\end{array}
$$

Note: (a) For triangular solid element NTYPE $=2$, set bulk modulus $B_{0}=0$.

(b) For triangular fluid element (NTYPE $=2$ ) or quadrilateral fluid element (NTYPE = 3) input density $p$ and bulk modulus $B_{0}$ only, set other variables equal to zero.

(c) If the quadrilateral elastic-plastic element is used to simulate fluid input density $\rho$, bulk modulus $B_{0}$ only. Set other variable equal to zero.

$5 y$

(6E10.4)

Material properties.

$$
1-10
$$$$
\text { E (7,MTYPE) }
$$

$v$ : Poisson's ratio.

$11-20$

E(8,MTYPE) $\quad B^{\prime}$ : The pressure derivative of the bulk modulus of elasticity, used for NTYPE $=4$ only.

$21-30$ E $(9, M T Y P E)$ Damping coefficient. 


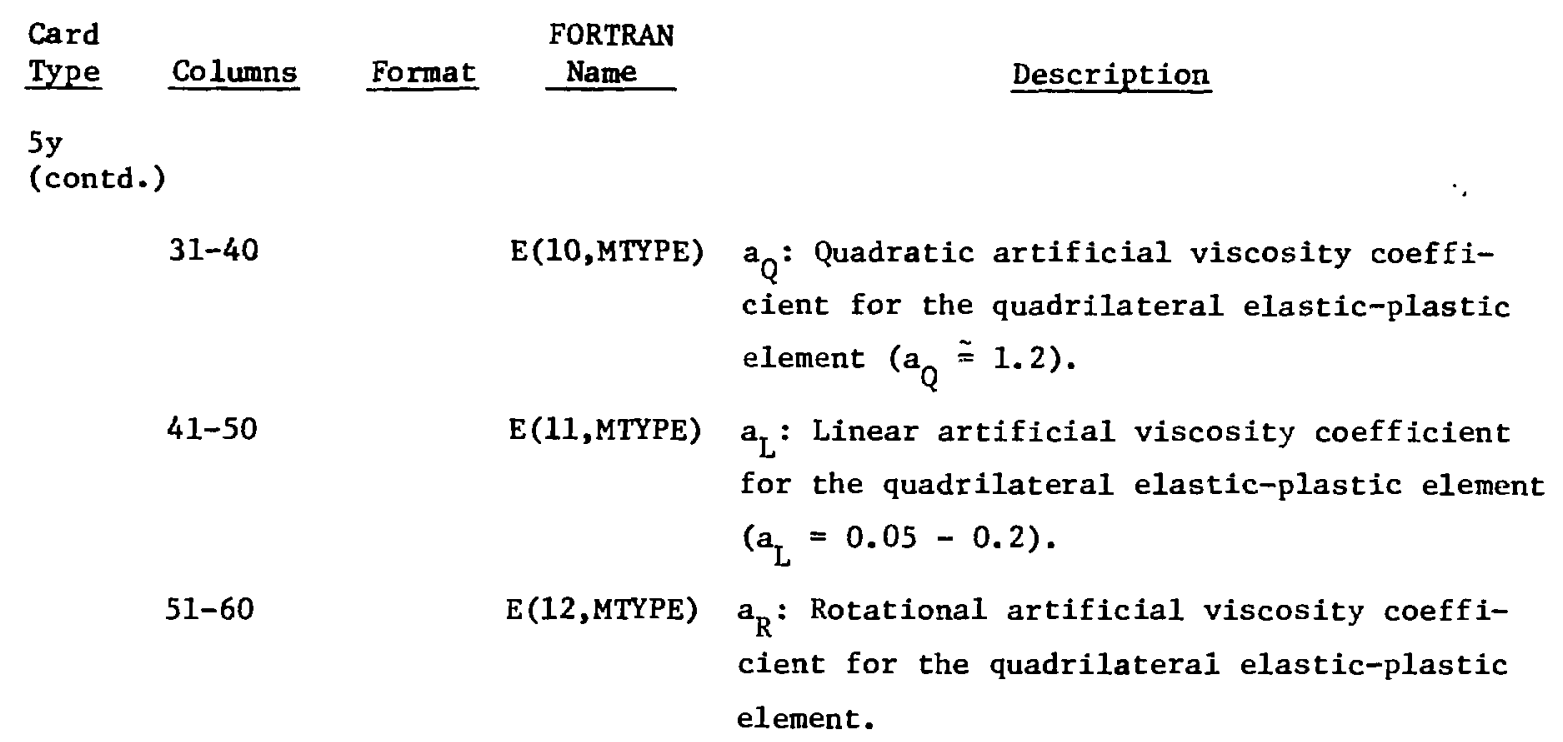

Note: (a) $\operatorname{ED}(9, \mathrm{MTYPE})$ through $\mathrm{ED}(12, \mathrm{MTYPE})$ are reserved as the damping coefficients for elements other than the quadrilateral elastic-plastic element.

(b) One should refer to REXCO-HEP, ANL-75-19 (Ref. 3) for damping coefficient and $a_{Q}$, $a_{L}$, as well as $a_{R}$ for the elastic-plastic element.

6

$$
(I 5,5 X, 2 E 10.4) \quad \text { Node cards. }
$$

1-5 N Node number.

11-20 XC(N) X-coordinate of real uodes. For "dumy" nodes (see NNODE description, card of Type 2) thickness of beam may be specified through $\mathrm{XC}(\mathrm{N})$. In that case all beam elements joined to node $\mathrm{N}$ will have the same thickness at that point.

21-30 $\mathrm{YC}(\mathrm{N}) \quad \mathrm{Y}$-coordinate of real nodes.

Note: Nodal coordinates for nodes equispaced between two nodes will be automatically generated if the data cards for intermediate nodes are skipped.

7

Element cards.
$1-5$
M
Element number.
NODE $(1, M)$ Node $\mathrm{N} 1$ of element (a "real" node). 


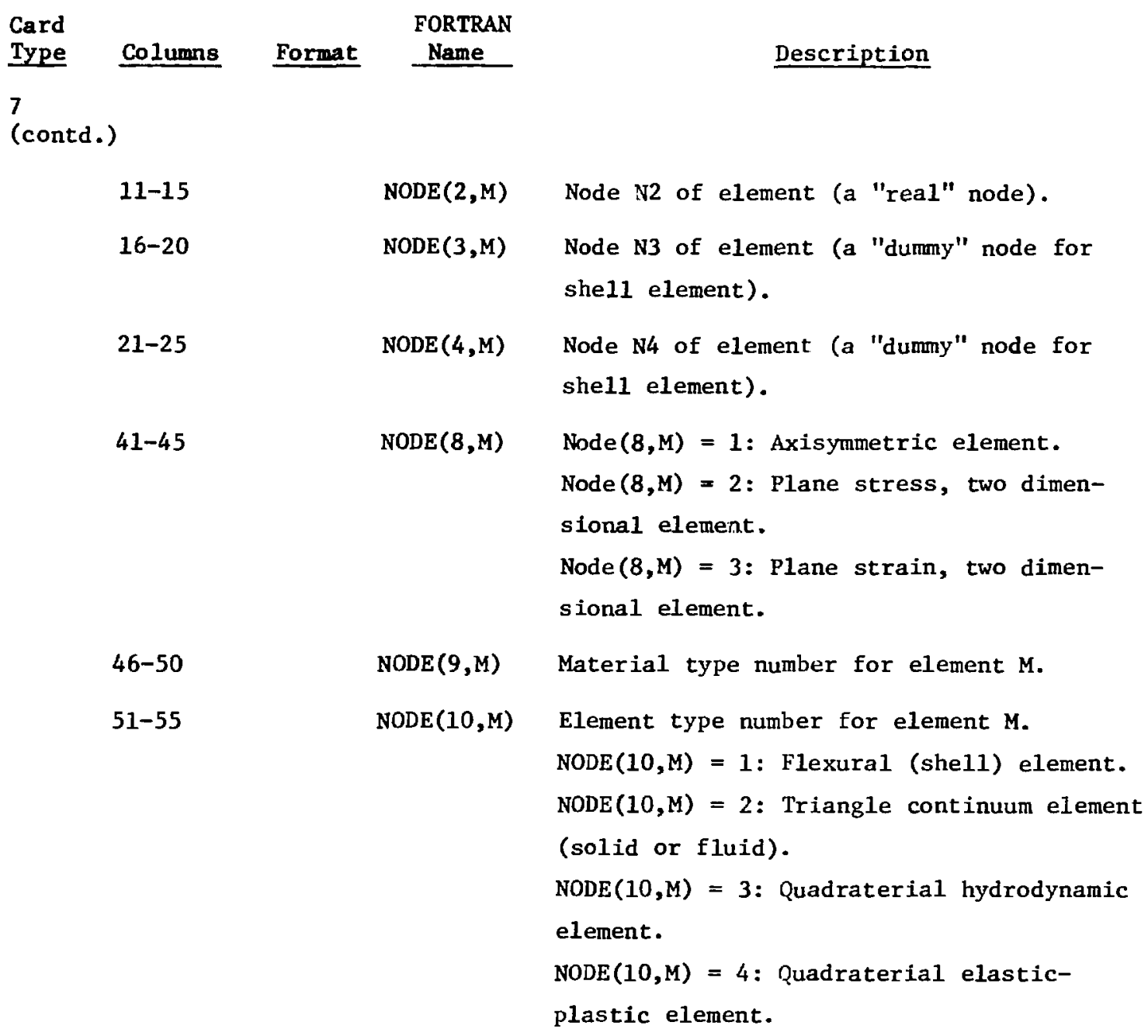

Note: Node cards for elements which can be generated by adding one to all node numbers of the previous element need not be included. The node data for these elements will be generated automatically. However, if this is done the node card for the last element must be included. NUMDIS cards (see NUMDIS on card Type 2 ).

N-7

The node at which one or more displacement components are prescribed to be zero or some non-zero value given in SUBROUTINE FREEFD. 


$\begin{array}{ll}\begin{array}{l}\text { Card } \\ \text { Type }\end{array} \text { Columns } \\ \text { (contd.) } & \begin{array}{l}\text { FORTRAN } \\ \text { Name }\end{array}\end{array}$

Note: (a) If NUMDIS $=0$, skip this type of cards.

(b) Use as many cards of Type 8 as required, total NUMDIS cards.

9

(4I10)

$1-10$

11-20

21-30

$31-40$
NPFREQ

NPRU

NPRS

NPIC output control card.

Output frequency; time history records of specified nodal and element information will be printed every NPFREQ main time steps.

Number of nodal time history records, i.e., displacements, velocities, or accelerations $0 \leq \mathrm{NPRU} \leq 20$. See card Type 10 .

Number of element time history records, 1.e., stresses, strains, etc. $0 \leq$ NPRS $\leq$

20. See card Type 11.

Number of complete output pictures at specified time steps; a complete picture consists 


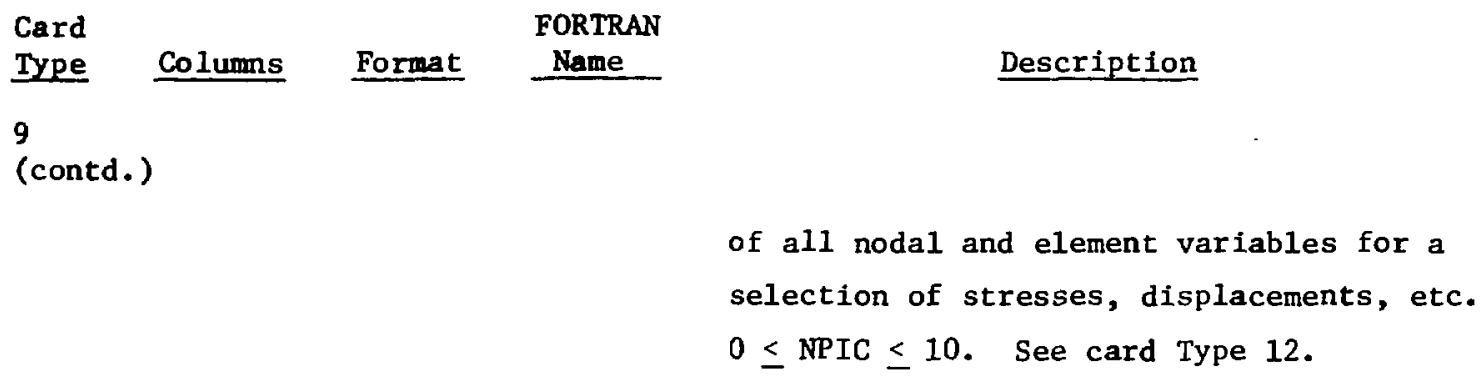

Note: (a) The plotting subroutine has been taken out from the structural dynamic program. Thus, it is suggested that NPFREQ, NPRU, NPRS, and NPIC be all set equal to zero. However, we retain the description in case we decide to put the plotting subroutine back in the program.

NPRU cards (see card 9). Use as many cards as needed, 8 items per card. If $\mathrm{NPRU}=0$, no cards of Type 10 are used. ment, velocity or acceleration time history is to be output, each NPFREQ cycles. Component number:

$L=1: x$-component (or rotatory component for dummy nodes).

$\mathrm{L}=2: \mathrm{y}$-component.

Nature of record:

$\mathrm{K}=0$ : Displacement record.

$\mathrm{K}=1:$ Velocity record.

$\mathrm{K}=2$ : Acceleration record .

10

Plot control:

$M=0$ : No plot.

$M=1$ : Nodal time history is plotted on printer only.

M = 2: Nodal time history is plotted on printer and CALCOMP.

$M=3$ : Nodal time history is plotted on printer and punched on cards. 


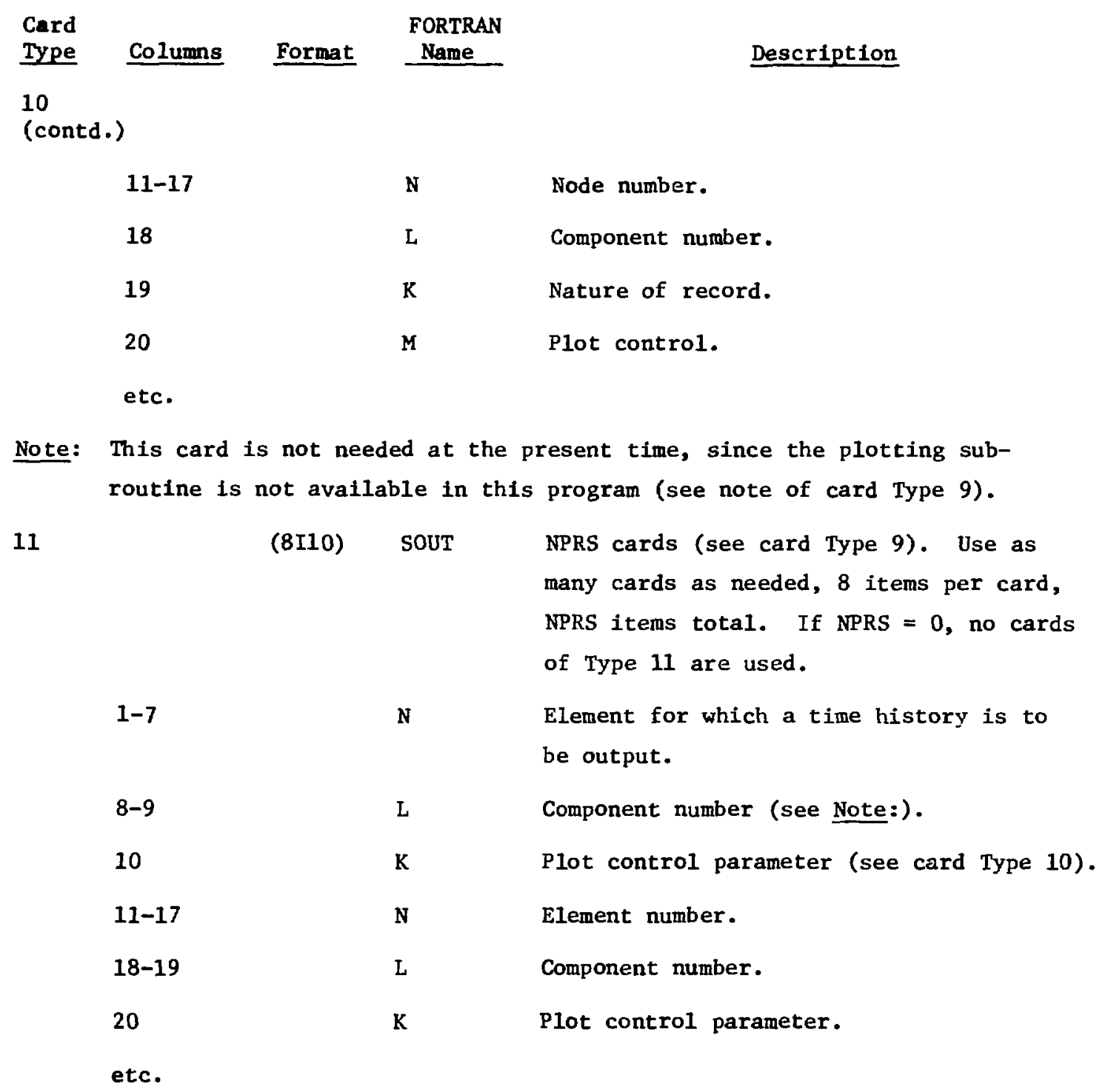

Note: (a) The component number (card Type 11, Cols. 8-9) is used to specify an element record as followe:

Plane element (triangle continuum, quadrilateral hydrodynamic, and elastic-plastic elements):

$L=1:$ Extensional strain $\hat{\varepsilon}_{\mathbf{x x}}$.

$L=2$ : Extensional strain $\hat{\varepsilon}_{y y^{*}}$

$L=3$ : Engineering shear strain $2 \varepsilon_{x y^{\circ}}$.

$L=4:$ Hoop strain $\varepsilon_{\theta \theta}$ (for axisymetric probleas). 
Card
Type Colums Format $\begin{aligned} & \text { FORTRAN } \\ & \text { Name }\end{aligned}$

11

(contd.)

$L=5$ to 8: Stresses $\sigma_{x}, \sigma_{y}, \sigma_{x y}$ and $\sigma_{\theta \theta}$ in the same order as the stresses of the flexural element.

Flexural element (beam and shell elements):

Since the integration for the nodal forces uses two points along the length and five points through the depth of an element. Figure 68 shows component numbers used to specify the element records. There are 50 records available for each flexural element.

(b) Card Type 11 is not needed at the present time (see note of card Type 9).

12

$1-10$

11-20
NPOUT

KONT
NPIC cards (see card Type 9). Use a number of cards of Type 12 equal to NPIC.

Time step at which a complete output as specified by KONT is desired.

KONT = 1: Output displacements at all nodes.

KONT $=2:$ Output above and coordinates of deformed structure (output of dummy nodes is meaningless for this card).

KONT $=3:$ Output above plus velocities and accelerations at all nodes.

KONT $=4:$ Output above plus all element information--stresses, strains, etc. KONT $=5$ : Same printed oritput as KONT $=2$; in addition, CALCOMP plot of the deformed structure.

KONT = 6: Same printed output as KONT = 3; in addition, CALCOMP plot of the deforwed structure. 


Card
Type Colums Eormat $\quad \begin{aligned} & \text { FORTRAN } \\ & \text { Name }\end{aligned}$ Description

12

(contd.)

KONT $=7:$ Same printed output as KONT $=4$; in addition, CALCOMP plot of the deformed structure.

Note: Card Type 12 is not needed at the present time (see note of card Type 9).

13

$\begin{array}{ll}1-5 & \text { IDUM } \\ 6-10 & \text { NDNOD }\end{array}$

11-15

$16-20$

INT1

21-25

INT2
Load line card description (see NPRES on card Type 2).

Load line number.

Number of points on load line (usually the number of "real" nodes on beam or she11).

$\operatorname{IVOL}(\mathrm{I}) \operatorname{IVOL}(\mathrm{I})=1$ : Load line is part of an axisymmetric surface.

IVOL $(I)=2:$ Load line is part of a plane surface (not available). I is the internal load number.

Internal increment between points on load line for automatic load line point generation. If INTI $>0$, the load line point is generated by adding INTI to the previous point number (NDNOD - 1) times starting with the first point specified on card Type 14. Set INT1 $=0$ if automatic generation is not desired. Usually INTI $=1$. Internal spacing between nodes on load line for automatic load line node generation. If INT2 >0, the load line node is generated by adding INT2 to the previous node number (NDNOD - 1) tines starting with the first node specified on card Type 15. Set INT2 $=0$, if autonatic generation is not desired. Usua11y INT2 $=1$. 
Card
Type Columns Format $\begin{gathered}\text { FORTRAN } \\ \text { Name }\end{gathered}$

13

(contd.)

26-30

INT3

(I6I5)

1-5

6-10

etc.

15

(I6I5)

1-5

6-10

etc.

16

(I6I5)

1-5

6-10

$t^{\prime}$

17

(3I5)
KPRES (J)

$\operatorname{KPRES}(\mathrm{J}+1)$

$\mathrm{J}=1$, NDNOD

KNODE (J)

iNNODE (J)

$J=1$, NDNOD

KSG (J)

$\mathrm{J}=1$, NDNOD

1-5

LM

Internal spacing between segments on load

line for automatic load line segments generation. If INT3 $>0$, the load line segment generated by adding INT3 to the previous segment number (NDNOD - 2) times starting with the first segment specified on card Type 16. Set INT3 $=0$, if automatic generation is not desired. Usually $\operatorname{IN} \Gamma 3=1$.

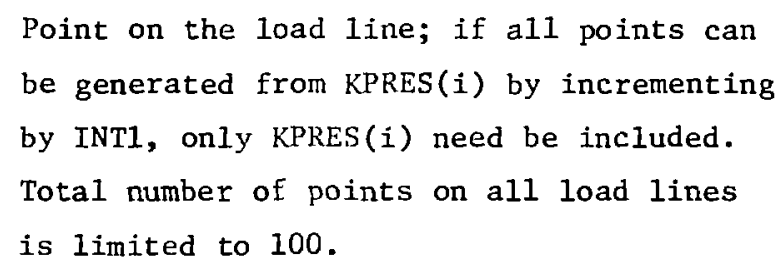

Segments on the load line; if all segments can be generated from KSG(i) by incrementing by INT3, only KSG(i) need be included. Total number of segments on all load lines is limited to 99 .

Segment/point connectivity cards.

Segment number. 
Card FORTRAN

Type Columns Format Name Description

17

(contd.)
$6-10$
IY $(1, L M)$
Point $i$ of segment.
$11-15$
IY $(2, L M)$
Point $j$ of segment.

Note: (a) Connectivity cards for segments which can be generated by adding one to all numbers of the previous segment need not be included. The data for these segments will be automatically generated. However, if this is done, the connectivity card for the last segment must be included.

(b) Card of Types 13 to 17 are to be used in sets, one set for each load line. Total number os sets is NPRES; $0<$ NPRES $\leq 10$. 


\section{CONCLUSIONS}

The ICECO-CEL (ICECO-III) Code is capable of providing accurate analysis of primary containment response to an HCDA. It can be used to analyze various transient phenomena, such as the wave propagation, slug impact, expanding gas bubble, fluid structure interaction, containment response, sodium spillage, liquid cavitation, and the above-core hydrodynamics. The code has several special features: (1) It can provide a stable solution throughout the entire excursion without using any complementary mechanisms such as artificial viscosities, rezoning, mesh stabilization and regularization, as well as prescribing grid motion. This is a deciding advantage for applying the code to the parametric studies and production runs. (2) It treats complex fluid motion in the complicated LMFBR containment and yet still maintains the computational efficiency. The computing time for analyzing the response of a typical LMFBR given in Sect. III.B. is about 20 minutes on an IBM 370/195, which is comparable to the conventional explicit numerical technique. (3) It has an accurate scheme for treating highly contorted coolant surfaces. Thus, the collapsing of the core-gas bubble at the early stage of excursion can be avoided and numerical calculations can be carried out continuously without any interruption. (4) The developed algorithm has the capability of treating flow through coolant passageways of the upper internal structure located in the above-core region. Thus, it can give an accurate estimate of tho mitigating effect of the upper internals on the slug-impact loads and the containment response, which is of significance in the safety analysis of LMFBRs. (5) It treats the sodium spillage through penetration openings on the reactor cover and the ruptured seal at the vessel-cover junction. This is not only important to the primary containment integrity, but is essential to the analysis of transport of radiological material to the secondary containment.

The accuracy of the code solutions have been validated against available experimental data, such as those obtained frum the ANL bubble experiment, SRI piplng test, SNR- 300 breeder reactor, UKAEA-JRC COVA experiment, and SRI complex vessel test SM-2. The good agreement between the code predictions and the recorded data demonstrates that the ICECO-CEL code is an accurate and useful tool for the LMFBR safety analysis. 


\section{ACKNOWLEDGMENTS}

The author would like to express his appreciation to S. H. Fistedis and $Y$. W. Chang for their constant encouragement and support. This work was performed in the Engineering Mechanics Program of the Reactor Analysis and Safety Division at Argonne National Laboratory, under the auspices of the $U$. $S$. Department of Energy. 


\section{REFERENCES}

1. S. H. Fistedis, "Containment of Fast Breeder Reactors - Present Status Remaining Problems," Proc. First Int1. Conf. on Structural Mechanics in Reactor Technology, Berlin, Germany, September 20-24, 1971.

2. Y. W. Chang, J. Gvildys, and S. H. Fistedis, "Two-Dimensional Hydrodynamics Analysis for Primary Containment," Argonne National Laboratory, ANL-74-98 (November, 1969).

3. Y. W. Chang, and J. Gvildys, "REXCO-HEP: A Two-Dimensional Computer Code for Calculating the Primary System Response in a Fast Reactor," Argonne National Laboratory, ANL-75-19 (June, 1975).

4. M. S. Cowler, "ASTARTE-A 2-D Lagrangian Code for Unsteady Compressible Flow, Theoretical Description," AWRE-44-91-37 (March, 1974).

5. K. Doerbecker, "ARES: Ein 2-Dim. Rechenprogramm Zur Beschreibung der Kurzzeit.igen Auswirkungen einer Hypothetischen Unkontrollierten Nuklearen Exkursion auf Reaktortank, Drehdeckel und Tankeinbanten, gezeigt am Beispiel des SNR 300," Reaktortagung Hamburg (April, 1972).

6. V. Adamik, "CEFRA: A Computational Programme for the Hydrodynamics Response of Primary Reactor Containment to Explosive Accident of Fast Reactor," Rep. of Power Research Institute - PRI (1973).

7. M. L. Wilkins, "Calculation of Elastic-Plastic Flow," UCRL-7322, Rev. 1 (1969).

8. B. J. Thorne, and W. Herrmann, "TOODY: A Computer Program for Calculating Problems of Motion in Two Dimensions," SC-RR-66-602 (July, 1967).

9. "PISCES 2DL Finite Different Equations 2DL-3," Physics International Co. (October, 1971).

10. R. Hofmann, "STEALTH: A Lagrangian Explicit Finite Difference Code for Solids, Structure, and Thermodynamic Analysis," EPRI NP-260 (August, I976).

11. C. Y. Wang, "ICECO - A Implicit Eulerian Method for Calculating Fluid Transient in Fast Reactor Containment," ANL-75-81 (December, 1975).

12. C. Y. Wang, J. C. Bratis, Y. W. Chang, and S. H. Fistedis, "Analysis of Fuel-Coolant Interactions by the Implicit Continuous Eulerian Containment Code," Trans. Am. Nuc1. Soc., Vol. 17, p. 355 (November, 1973).

13. C. Y. Wang, "Analysis of Nonlinear Fluid-Structure Interaction Transient in Fast Reactors," ANL-78-103 (November, 1978).

14. C. Y. Wang, "Comparison of ICECO Code Predictions with Flexible Vesse1 Experiment," Nucl. Eng. Design, Vol. 49, p. 145 (July, 1978).

15. F. H. Harlow, and A. A. Amsden, "A Numerical Fluid Dynamics Calculation Method for Al1 Flow Speeds," J. Comp. Phys., Vol. ㅈ, p. 197 (1971). 
16. W. Prager, "Introduction to Mechanics of Continua," Ginn and Company, Boston, U.S.A. (1961).

17. W. T. Sha, et al, "COMIX-1: A Three-Dimensional Transient Single-Phase Component Computer Program for Thermal-Hydrodynamic Analysis," NUREG/GR0785, ANL-77-96 (1978).

18. H. Y. Chu, "A Quasi-Eulerian Method for Analyzing Slug Impact and Coolant Spillage in a Fast Reactor Accident," ANL/RDP 76-14 (1976), later published as ANL-79-89 (1979).

19. J. A. Viecelli, "A Computing Method for Incompressible Flows Bounded by Arbitrary Walls," J. Comp. Phys., Vol. 8, pp. 119-143 (1971).

20. B. D. Nichols, and C. W. Hirt, "Improved Free Surface Boundary Conditions for Numerical Incompressible-Flow Calculations," J. Comp. Phys., Vo1. 8, p. 438 (1971).

21. S. L. Hancock, "Application of a Coupled Euler-Lagrange Computer Program to the Structural Response of an LMFBR," Nucl. Eng. Design, Vol. 42, p. 69 (1977).

22. U.S. Department of Energy, "The APRICOT Program: Comparison and Benchmarking of Computational Methods for Analysis of LMFBR Structural Response to Postulated Core Disruptive Accidents," SAN-1112-1 (October, 1977).

23. A. V. Jones, "The Mitigating Effect of Coolant Cavitation in the Response of LMFBR Containment to a Whole-Core Accident," Proc. Intl. Meeting on Fast Reactor Safety and Related Physics, Vol. III, Chicago, October 5-8, 1976.

24. M. S. Cowler, and S. L. Hancock, "The Numerical Calculation of Stress Transients in Cavitated Fluids," Private Communication (August, 1979).

25. D. H. Cho, and R. W. Wright, "A Rate-Limited Model of Molten Fuel-Coolant Interactions," Trans. Am. Nucl. Soc., Vol. 13, p. 658 (1970).

26. T. J. Marciniak, "Heat Transfer in Postburst Accident Models," Proc. First Int1. Conf. on Structural Mechanics in Reactor Technology, Berlin, September 20-24, 1971. Vol. III, Reactor Components, Part E, Shock and Vibration Analysis of Reactor Components, Rept. EUR $4820 \mathrm{~d}-\mathrm{f}-\mathrm{e}$, PP. 189206.

27. J. C. Bratis, T. J. Marciniak, and J. Gvildys, "REXCO-HT: A Hydrodynamic Containment Code Including Molten-Fuel-Coolant Interactions," ANL-75-15 (February, 1975).

28. C. W. Hirt, "Heuristic Stability Theory for Finite Difference Equations," J. Comp. Phys., Vol. 2, p. 339 (1968).

29. T. Belytschko, and B. J. Hsieh, "Nonlinear Transient Analysis of Shells and Solids of Revolution by Convected Elements," AIAA, Vol. 12, No. 8, p. 1031 (1974). 
30. T. Belytschko, "WHAMS-Waves in Hysteretic Arbitrary Media and Structures," Dept. of Material Engineering, University of Illinois at Chicago Circle (1974).

31. T. Belytschko, and B. J. Hsieh, "Nonlinear Transient Finite Element Analysis with Convected Coordinates," Int1. J. for Num. Meth. in Eng., Vol. 7 , pp. 255-271 (1973).

32. R. F. Kulak, Private Communication, (1977).

33. A. L. Florence, and G. R. Abrahamson, "Simulation of a Hypothetical Core Disruptive Accident in a Fast Flux Test Facility," HEDL-SRI-1 (May, 1973).

34. M. Egléme, et al, "Nuclear Accident Simulation Programme, Part III Experiments in $1 / 13$ Scale Vessels at JRC - Ispra (Italy)," Topical Report BN 7510-01 (October, 1975).

35. N. J. M. Rees, R. B. Tattersa11, and G. Verzelletti, "Results of Repeat Firings of High Explosive Charges in Water-Filled Vessels," Joint Report by AWRE, UK, and UKAEA, AEE, and EURATON, JRC, Report No. AWRE/44/97/1, TGR Report 2909 (R/X), JRC ISPRA EE/01/76, AEEW M-1417 (August, 1976).

36. W. Herrmann, and N. E. Hoskin, "APRICOT Code Comparisons - Results of Phase 2," Private Communication (November, 1978).

37. C. Y. Wang, "Analysis of High Energy Excursions Using the Implicit ContinuousFluid Eulerian Containment Code (ICECO)," Nucl. Technol. 51, 400-413 (1980).

38. C. M. Romander, and D. J. Cagliostro, "Structural Response of a 1/20-Scale Models of the Clinch River Breeder Reactor to a Simulated Hypothetical Core Disruptive Accident," SRI International Technical Report 4 (October, 1978).

39. J. E. Welch, et al, "The MAC Method, A Computing Technique for Solving Viscous, Incompressible, Transient Fluid-Flow Problems Involving Free Surface," LA-3425 (1966).

40. A. A. Amsden, and F. H. Harlow, "The SMAC Method: A Numerical Technique for Calcuiating Incompressible Fluid Flows," LA-4370 (1970). 


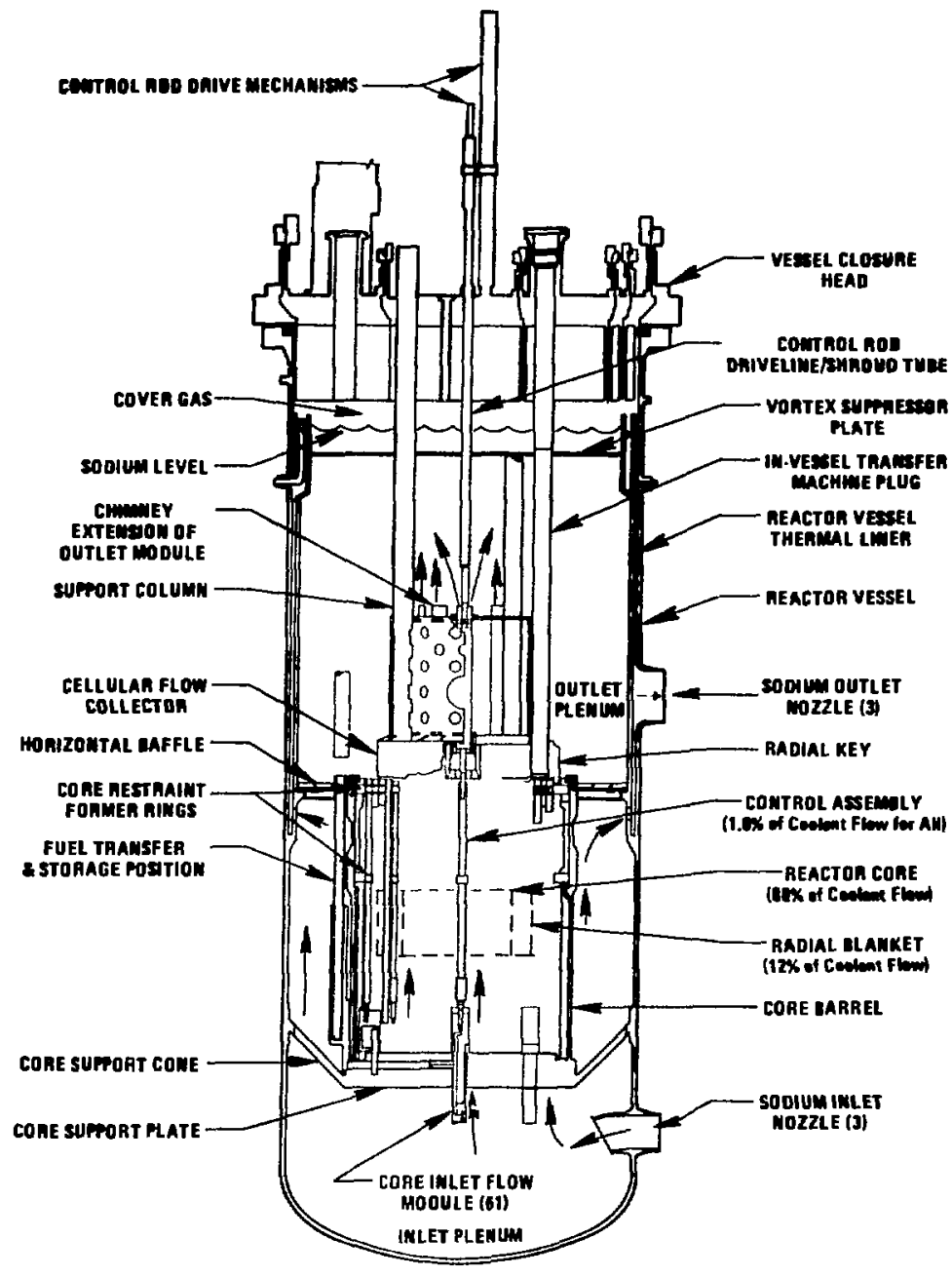

Fig. 1. Typical Configuration of an LMFBR

$\operatorname{CELL}(i, j)$

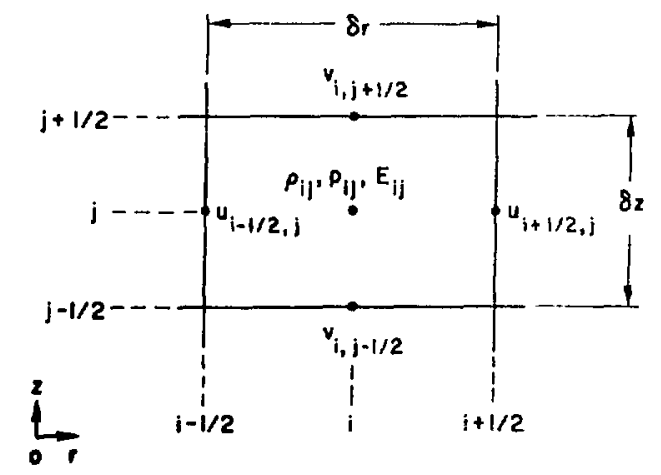

Fig. 2

The location of the Cell Variables in an Eulerian Computational Cell 

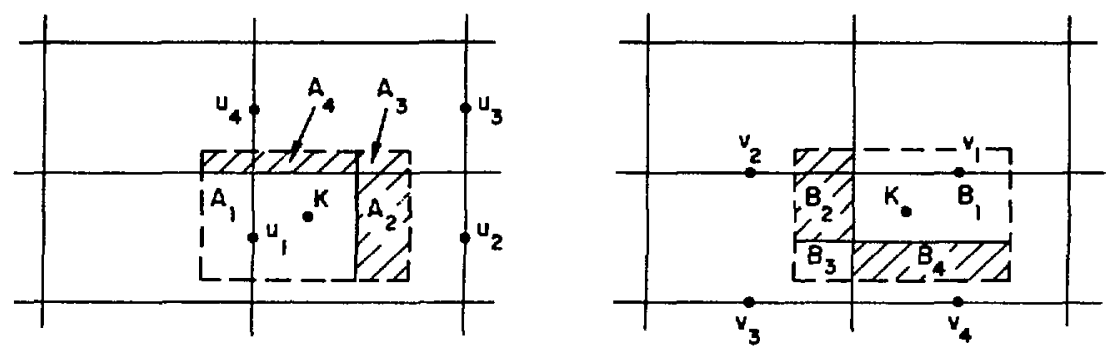

Fig. 3. Linear Interpolation for $\mathrm{U}_{\mathrm{K}}$ and $\mathrm{V}_{\mathrm{K}}$ with Position of $\mathrm{K}^{\text {th }}$ Particle as Shown

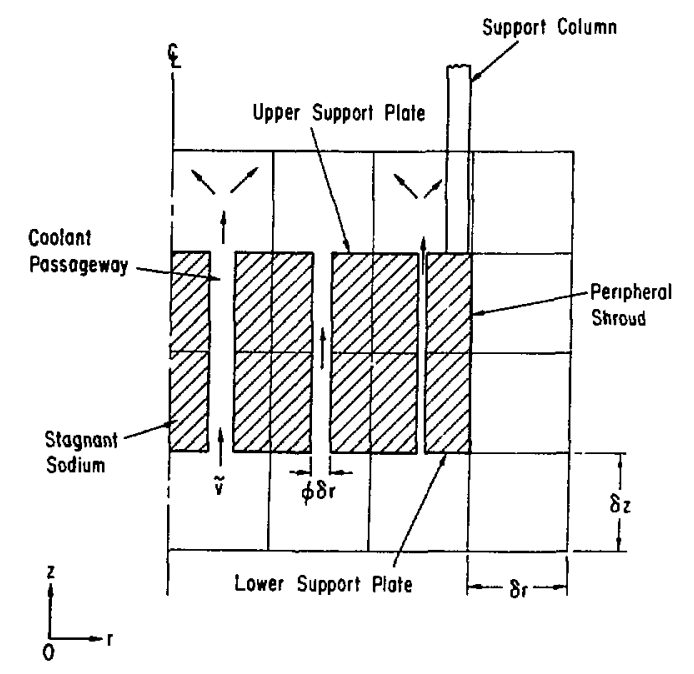

Fig. 4

Eulcrian Computing Mesh for Upper Internal Structure
Fig. 5

Control Volume for Derivation of Axial Momentum Equation Inside Coolant Passageways

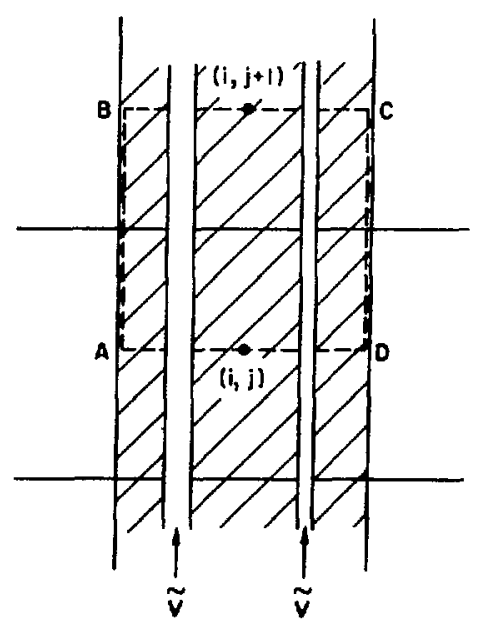



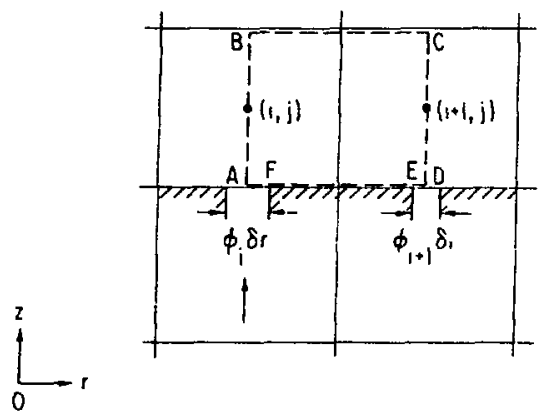

Fig. 6. Control Volume for Derivation of Radial Momentum Equation A bove the Perforated Structure

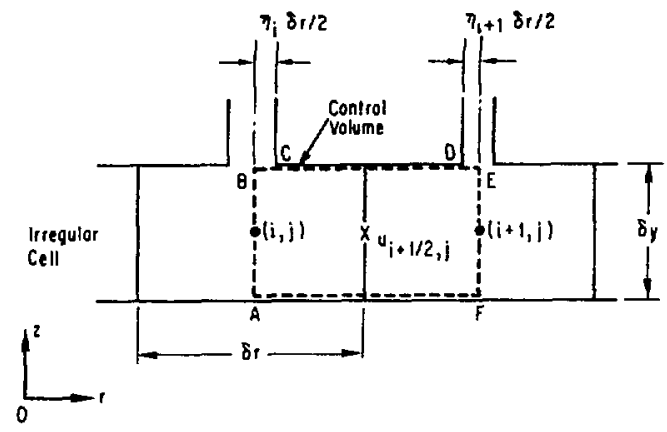

Fig. 8. Control Volume for Radial Momentum Equation Underneath the Cover

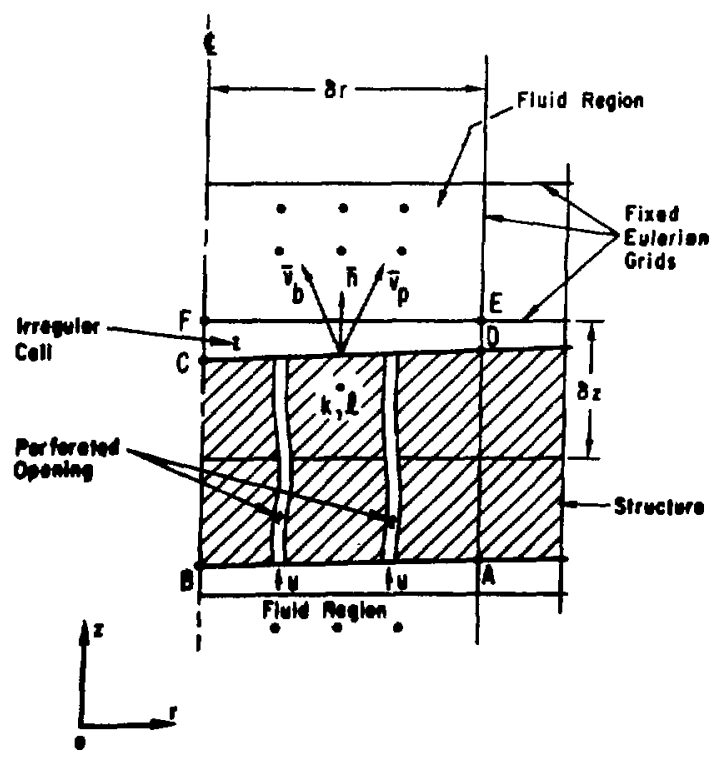

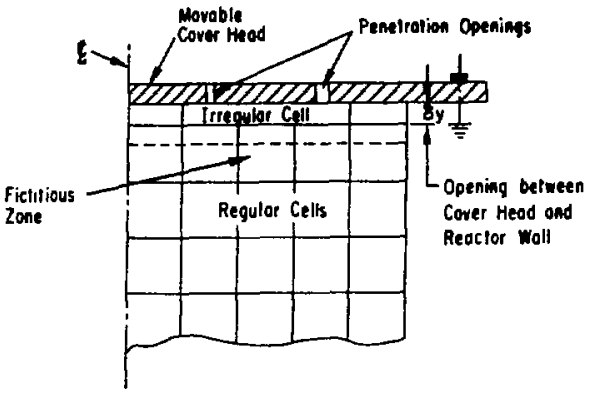

Fig. 7. Reactor Cover with Penetration Openings

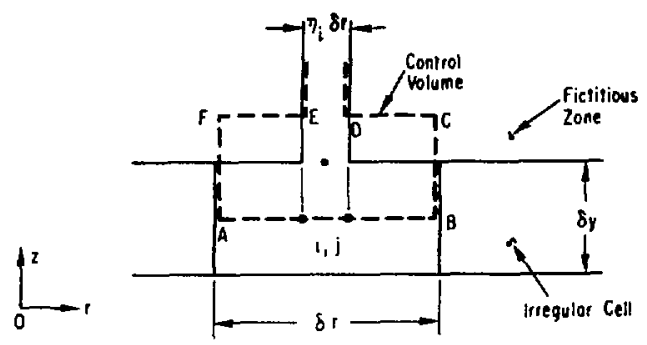

Fig. 9. Control Volume for Axial Momentum Equation Underneath the Cover

Fig. 10

Irregular Cell $(k, \ell)$ Containing Deformable Structure with Perforated Openings 


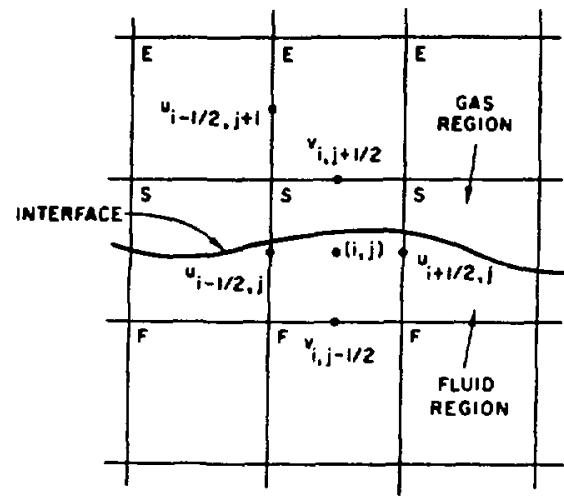

Fig. 11

Velocity Calculation in Vicinity of a Surface Cell

1. EMPTY CELL

S: SURFACE CELL

F: FULL CELL

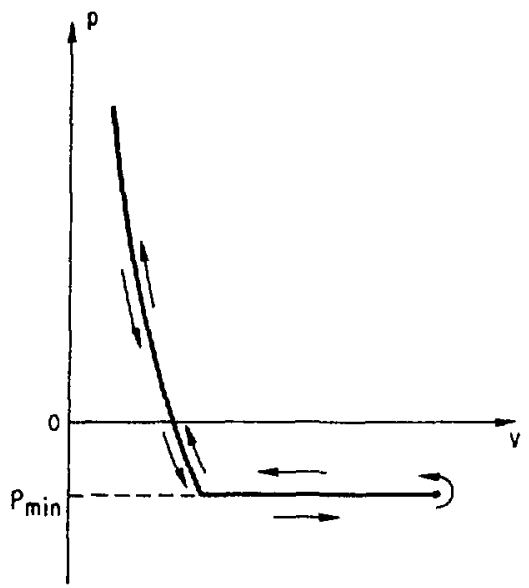

(o)

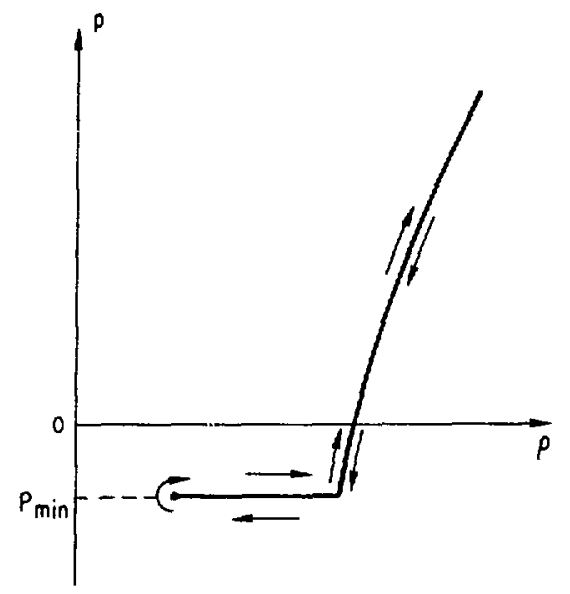

(b)

Fig. 12. Conventional Equation of State for Cavitated Fluid

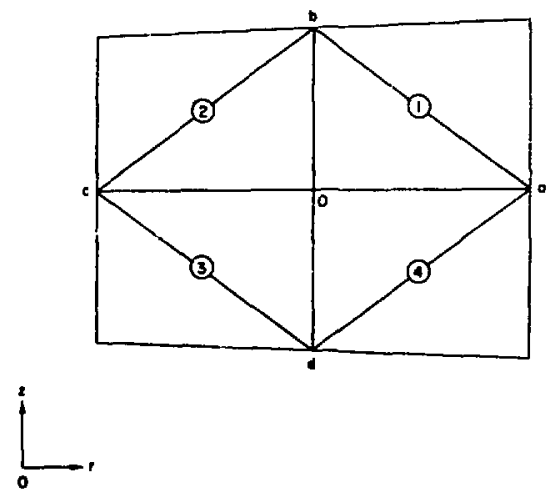

Fig. 13. Zone Configuration for FiniteDifference Equations
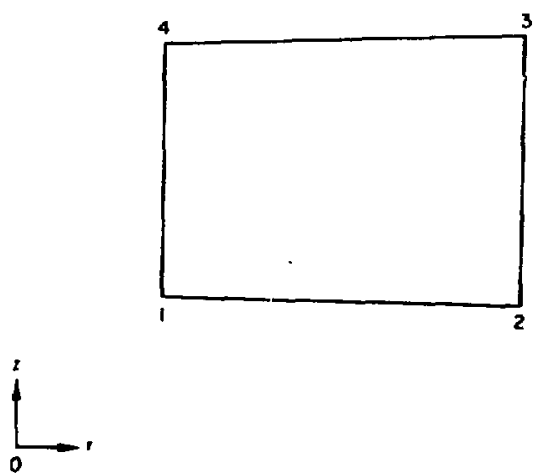

Fig. 14. Numbering Scheme for Calculating Node Forces 


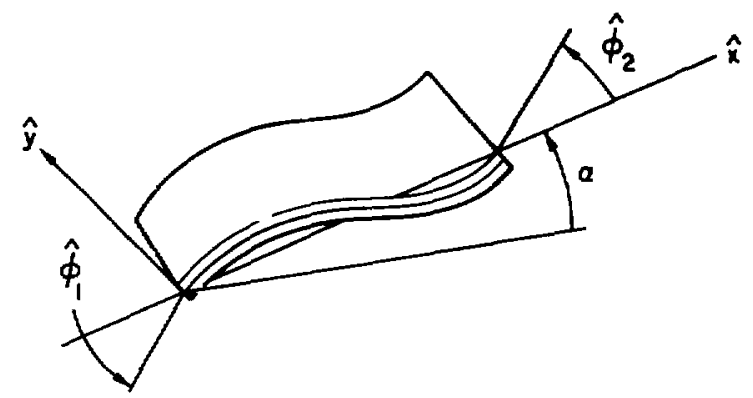

Fig. 15

Axisymmetric Shell Element
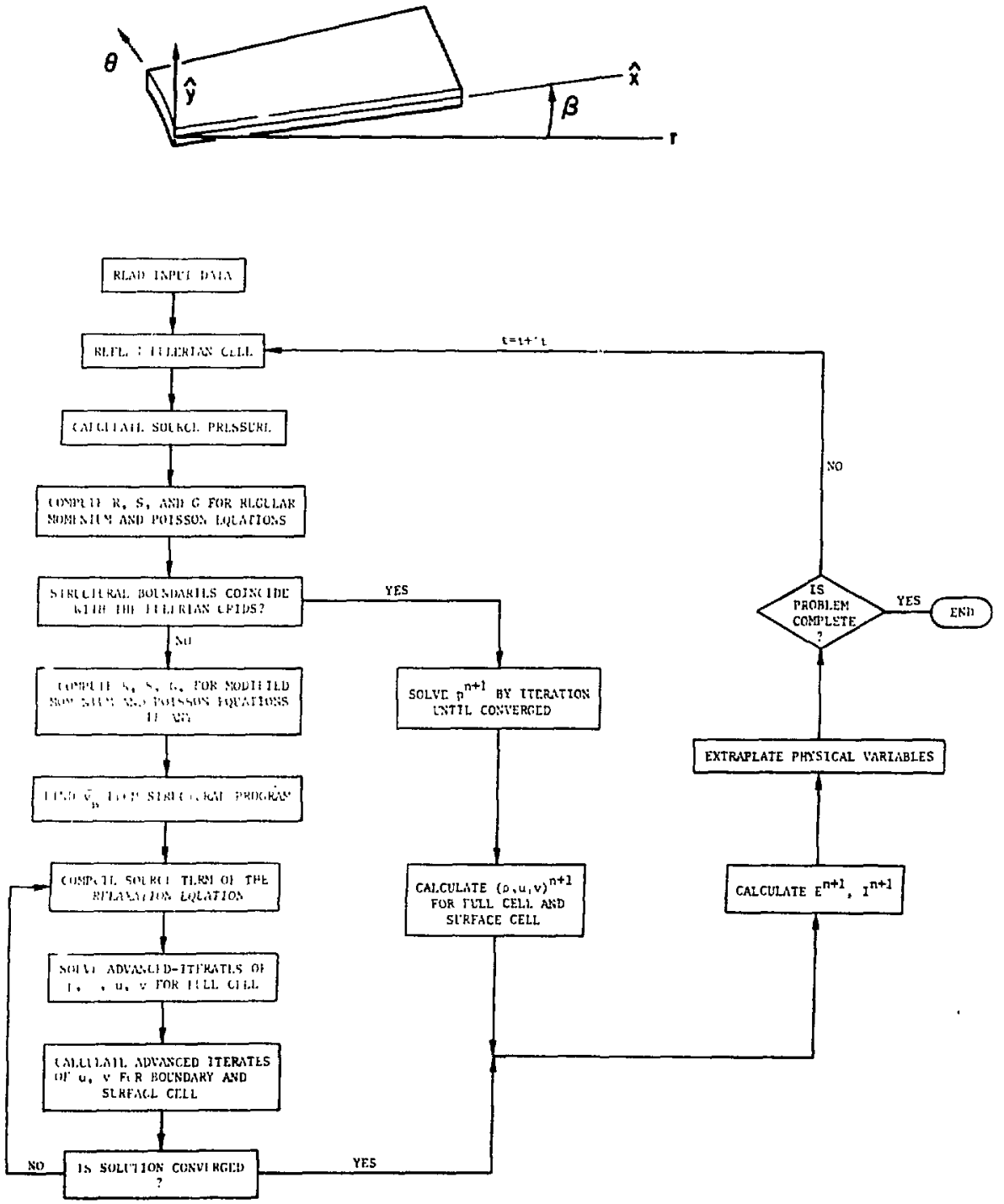

Fig. 16. Flow Chart Representing the Computational Procedure 


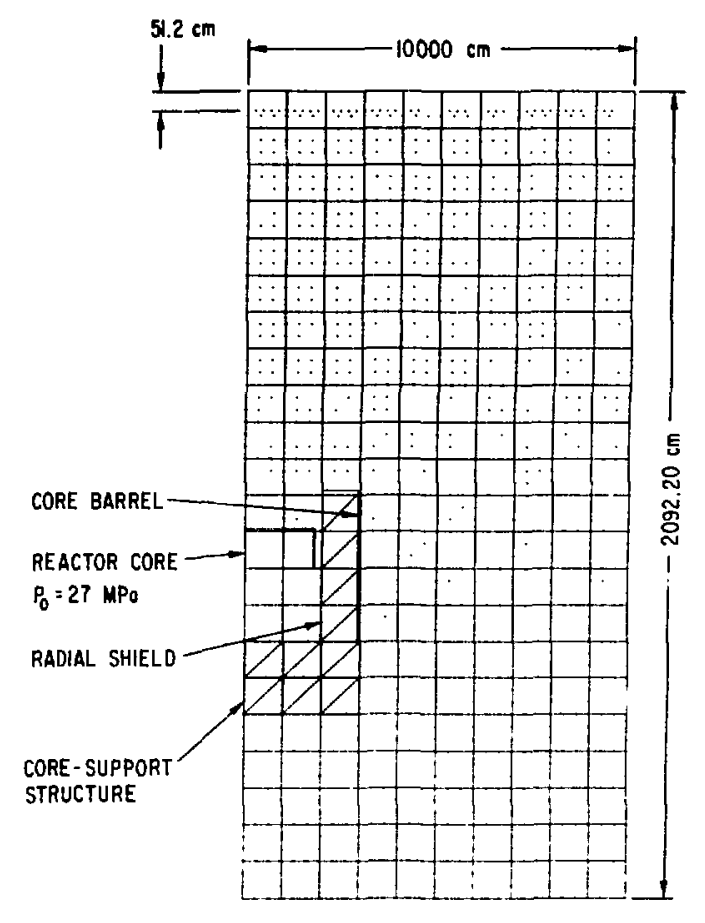

Fig. 17

Initial Configuration of the Pool-Type LMFBR

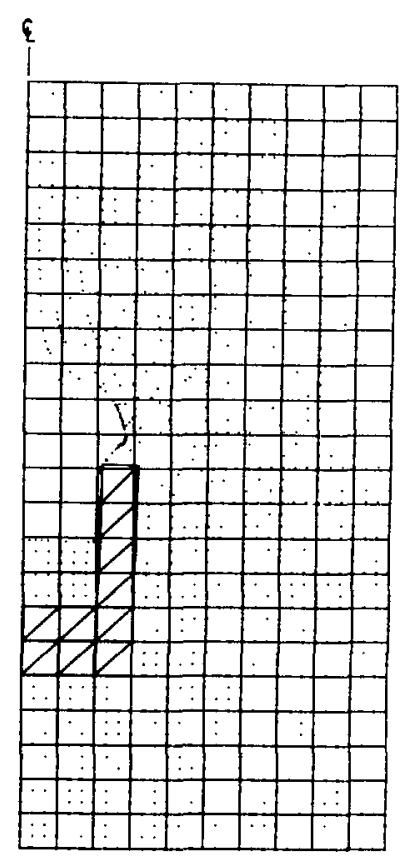

$t=52 \mathrm{~ms}$

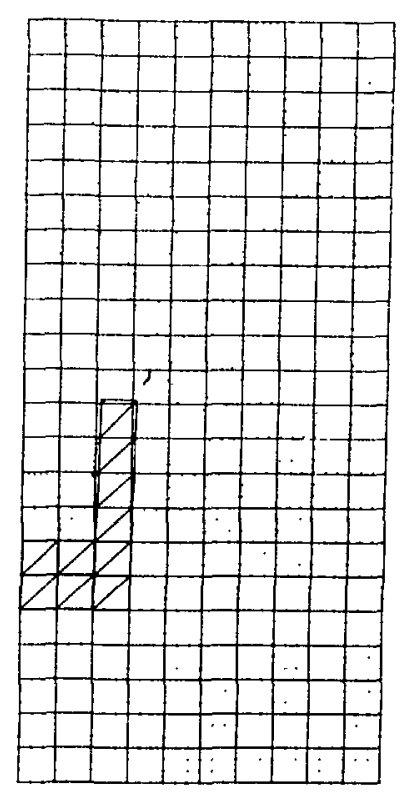

$\mathrm{t}=72 \mathrm{~ms}$

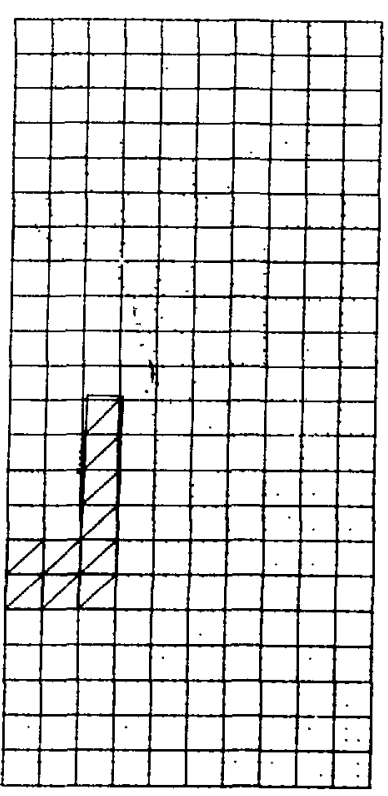

$1=87 \mathrm{~ms}$

Fig. 18. Reactor Configurations at Several Different Times 


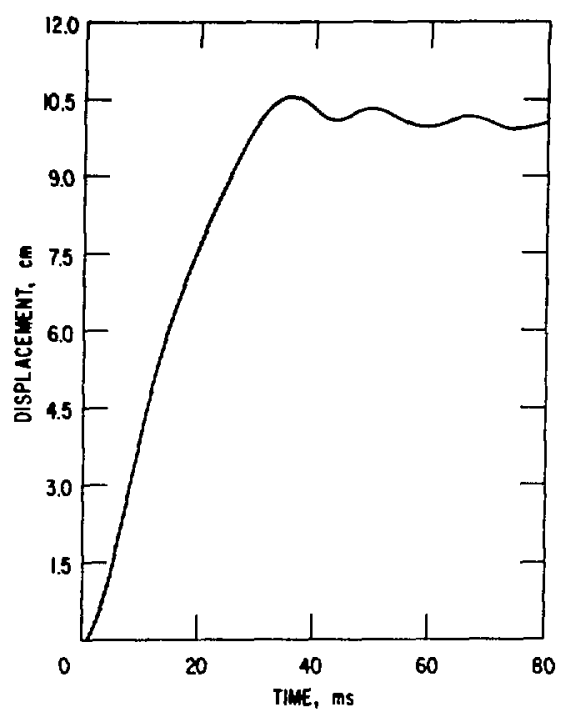

rig. 19

Radial Displacement History of the Upper End of the Core Barrel

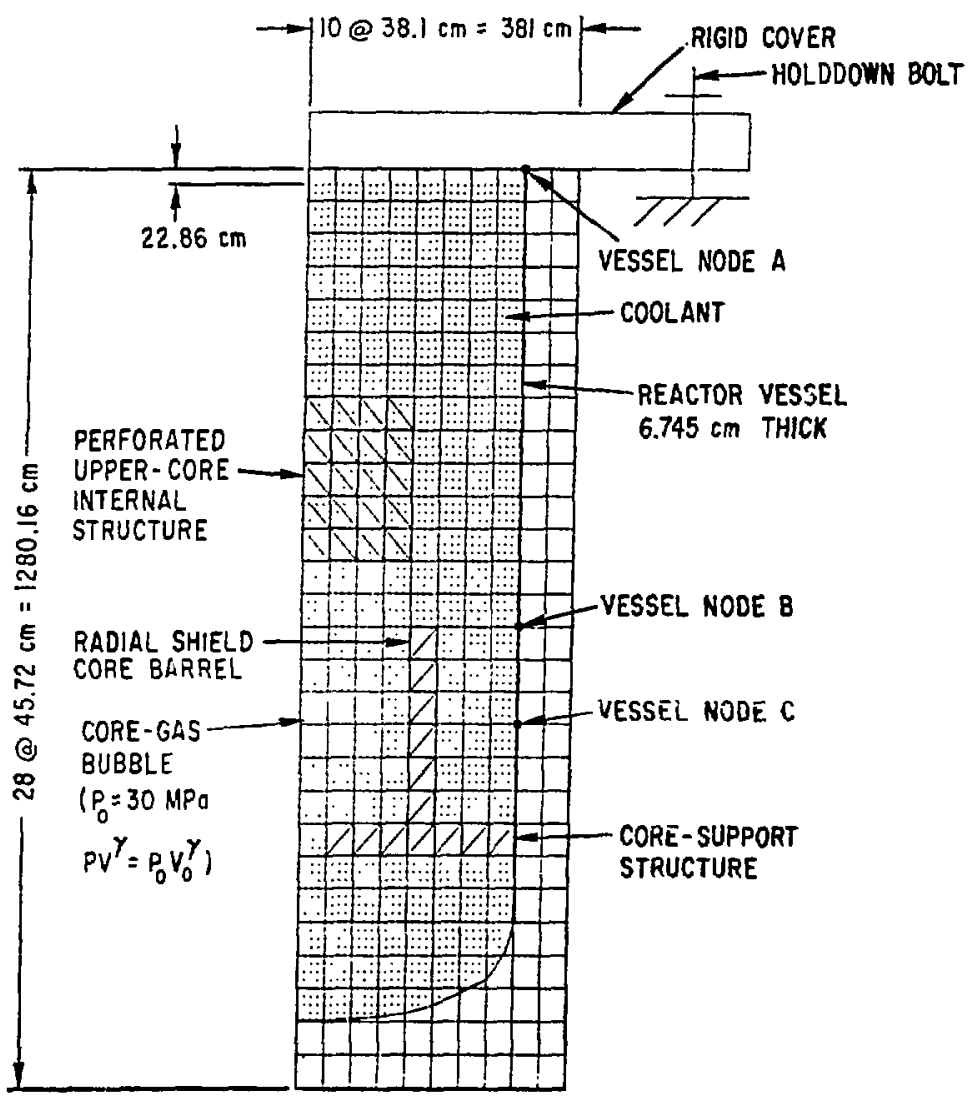

Fig. 20. Initial Reactor Configuration with the Upper Internal Structure 


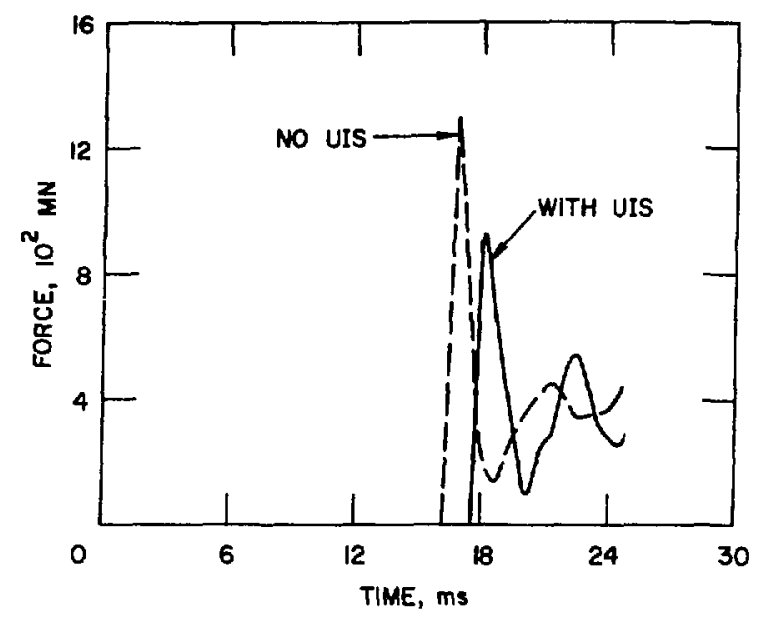

Fig. 21

Comparison of Total Force on the Reactor Cover

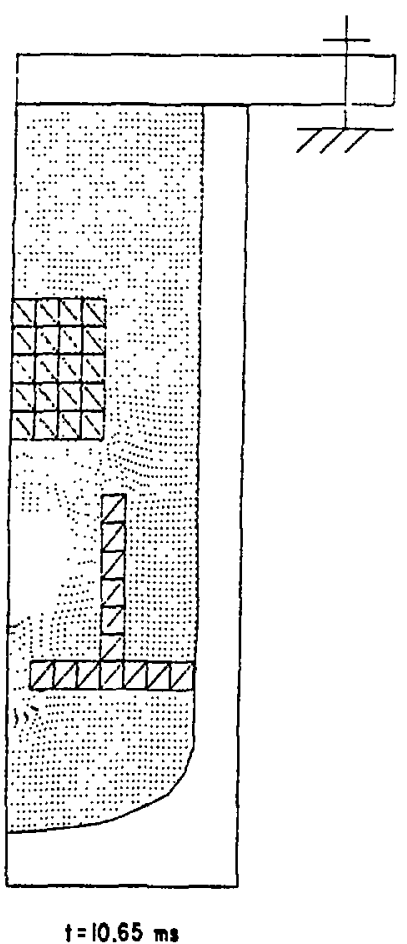

(0)

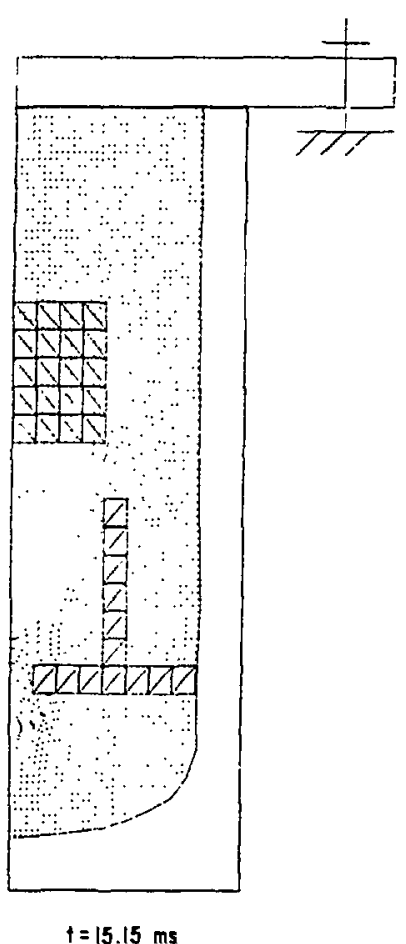

(b)

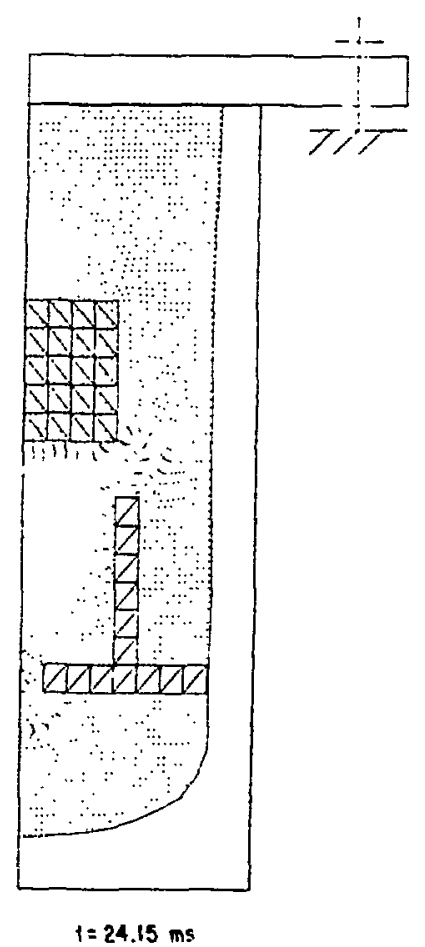

(c)

Fig. 22. Reactor Configurations at Three Different Times (Upper Internal Modeled as Perforated Structure) 


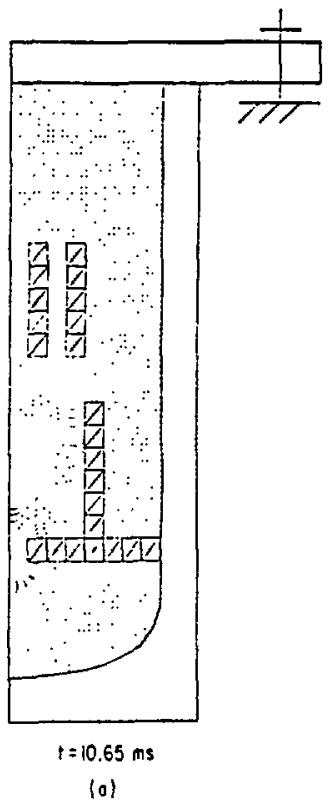

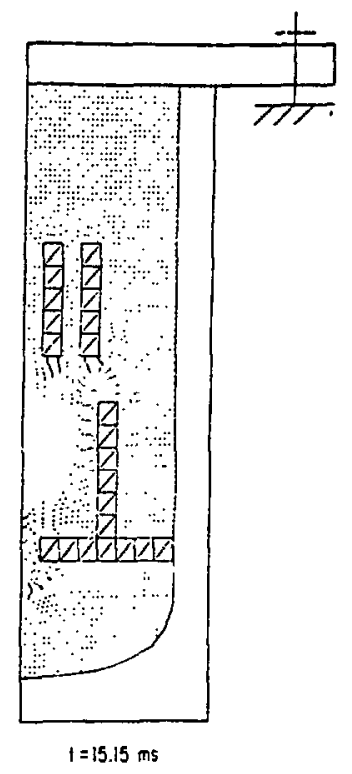

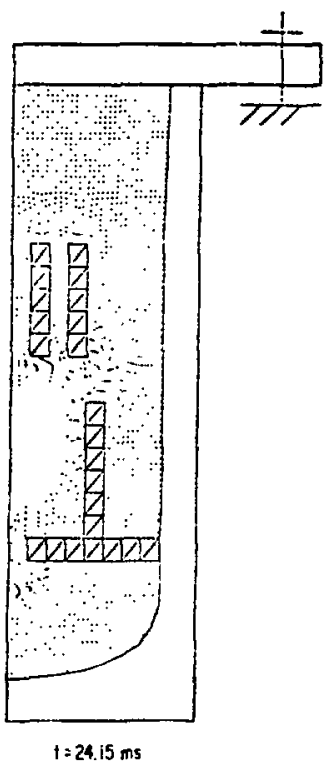

(c)

Fig. 23. Reactor Configurations at Three Different Times (Upper Internal Mlodeled by Rigid Obstacles)

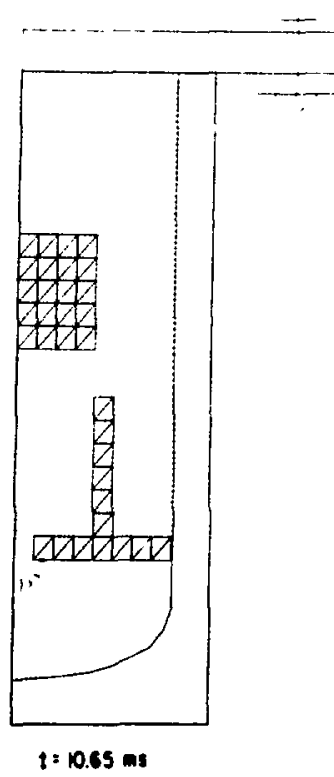

(1)

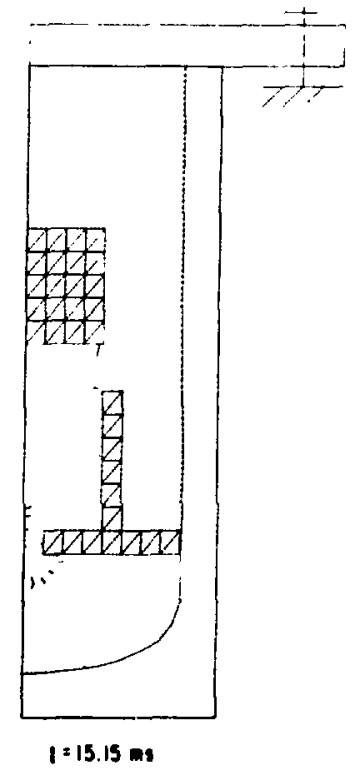

(b)

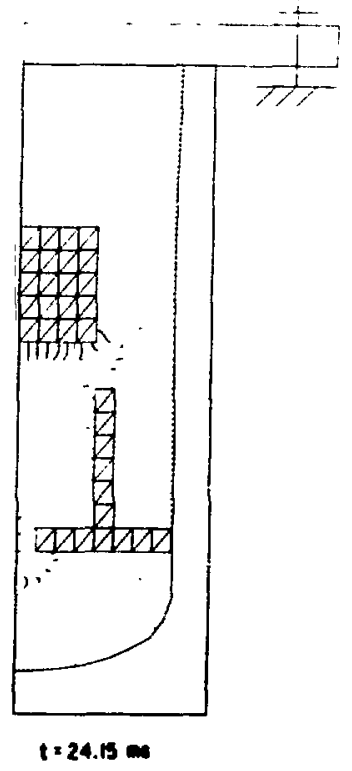

(e)

Fig. 24. Reactor Configurations at Three Different Times (UIS Passageways Were Plugged) 


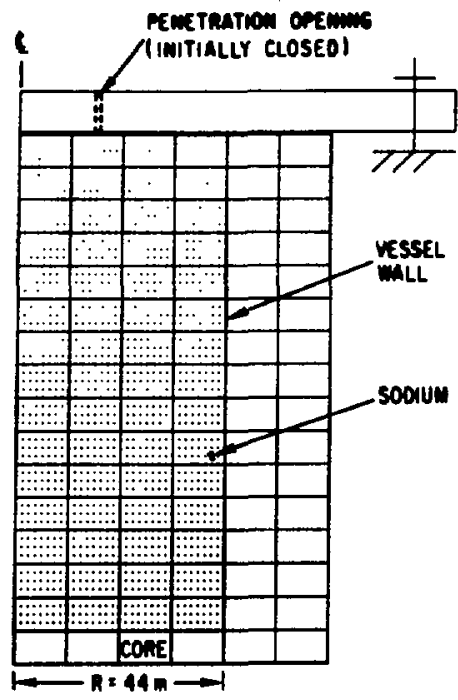

(a) $t=0$

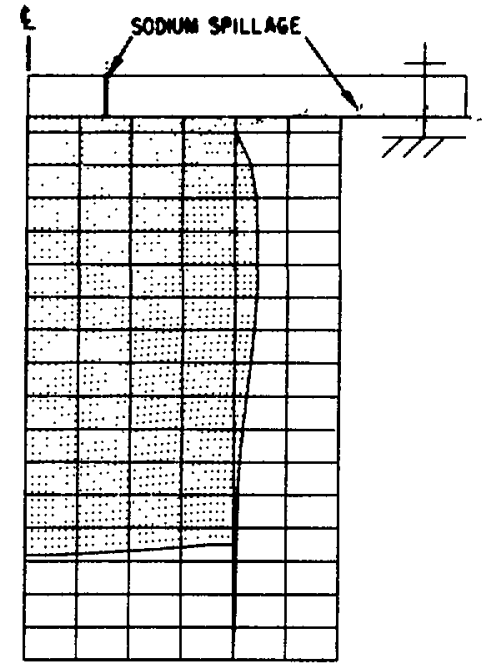

(b) $1=50 \mathrm{~ms}$

Fig. 25. (a) Configuration of a Simpli fied Reactor (Top Penetration Initially Closed), (b) Configuration of $50 \mathrm{~ms}$ Showing Coolant Spillage from Top Penetration and from Vessel-Heat Junction

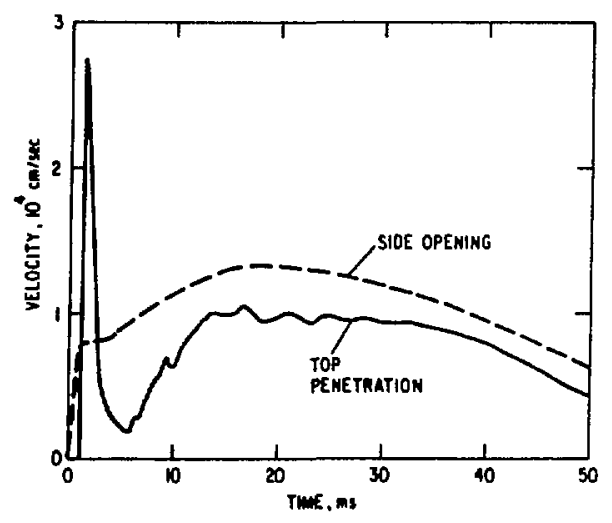

Fig. 26

Sodium Velocities at Top Penetration and Side Opening

Fig. 27

Total Spillage from Top Penetration and Side Opening

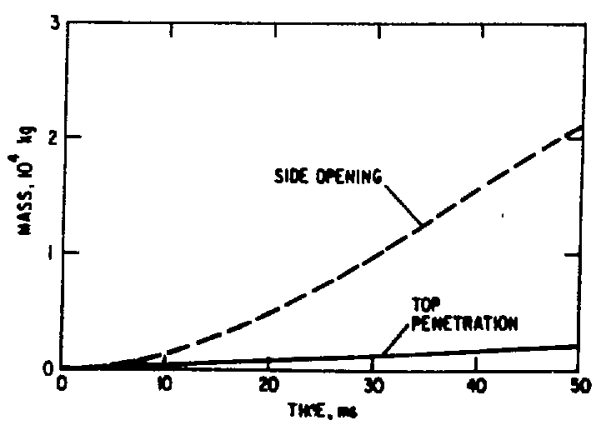




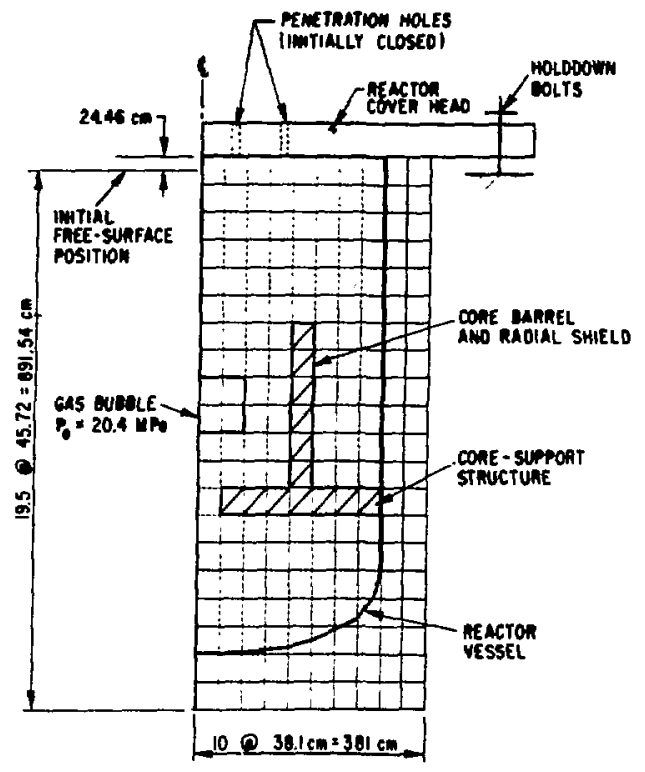

Fig. 28

Initial Configuration Used for Contaisment and Sodium-Spillage Analysis

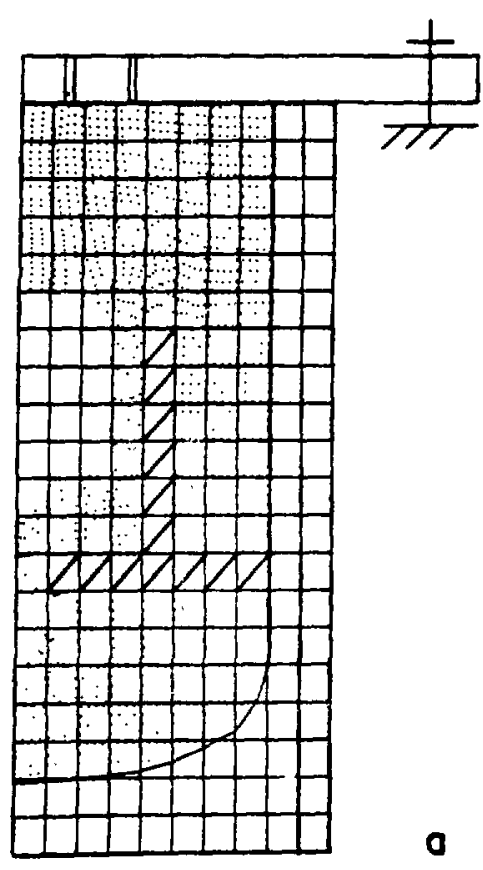

$t=18.9 \mathrm{~ms}$

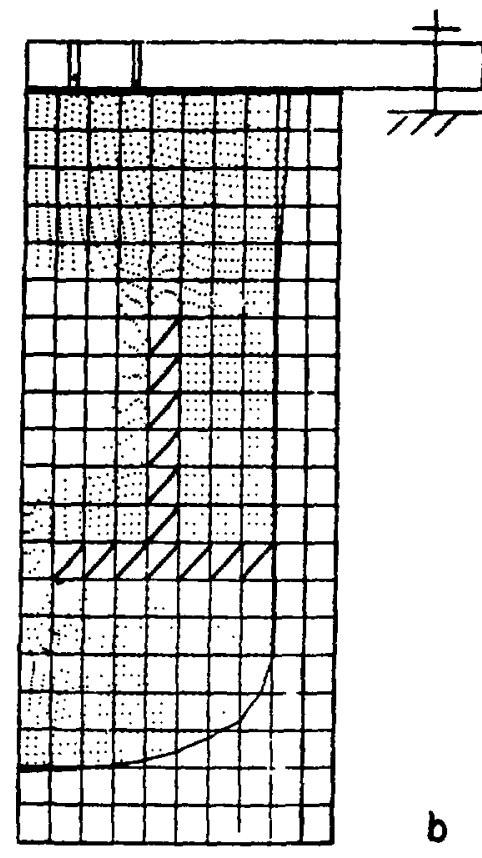

$t=22.6 \mathrm{~ms}$

Fig. 29. Reactor Configurations at Two Different Times 


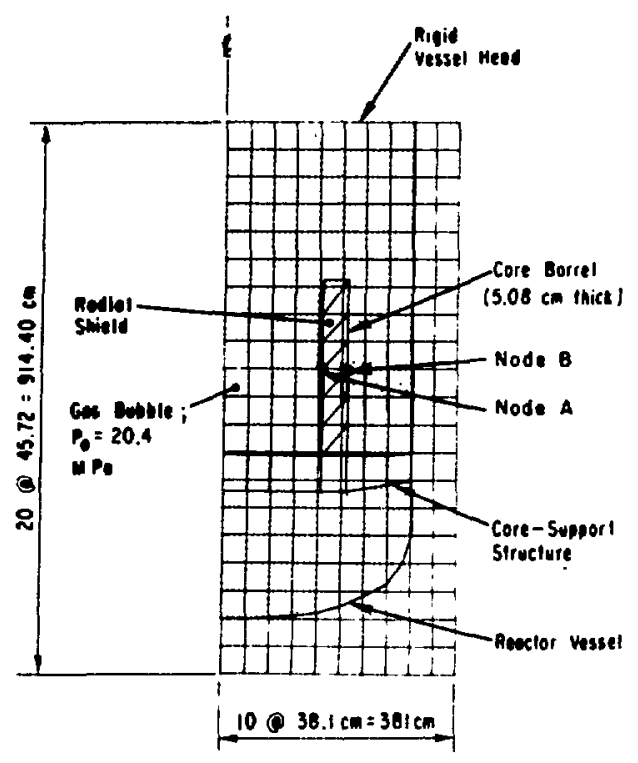

Fig. 30

Initial Configuration Used in the Analysis

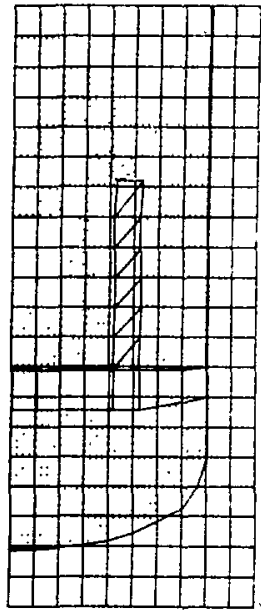

$t=8.3 \mathrm{~ms}$

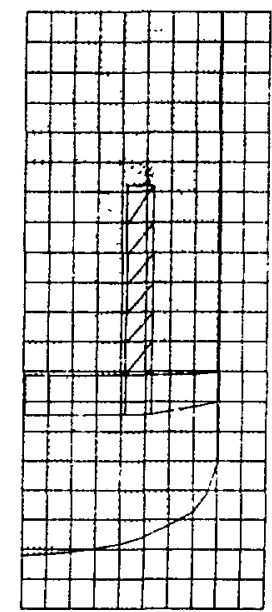

$t=14.3 \mathrm{~ms}$

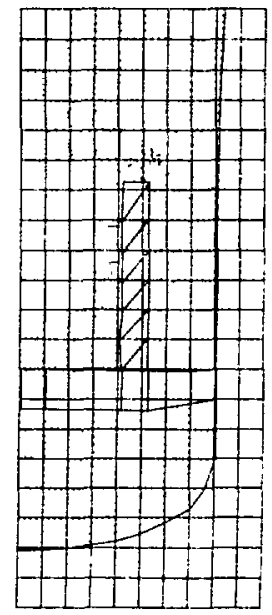

$t=20.3 \mathrm{~ms}$

Fig. 31. Configurations at Various Times

Fig. 32

Pressure Loadings in the Reactor Lower Plenum

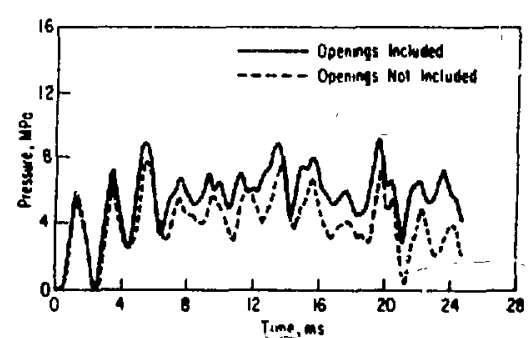




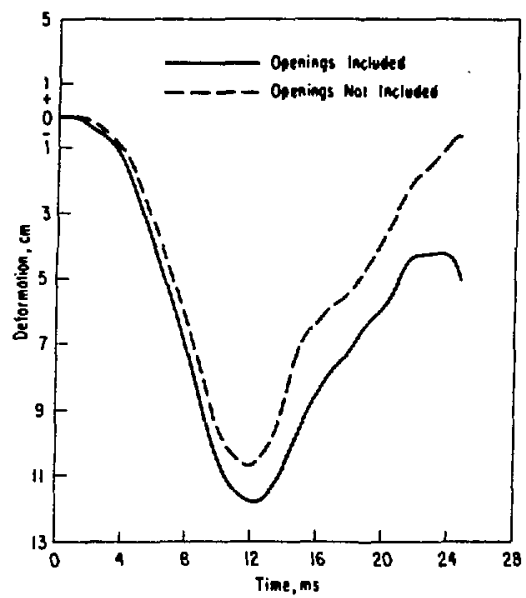

Fig. 33

Axial Deformation of Bottom Vessel
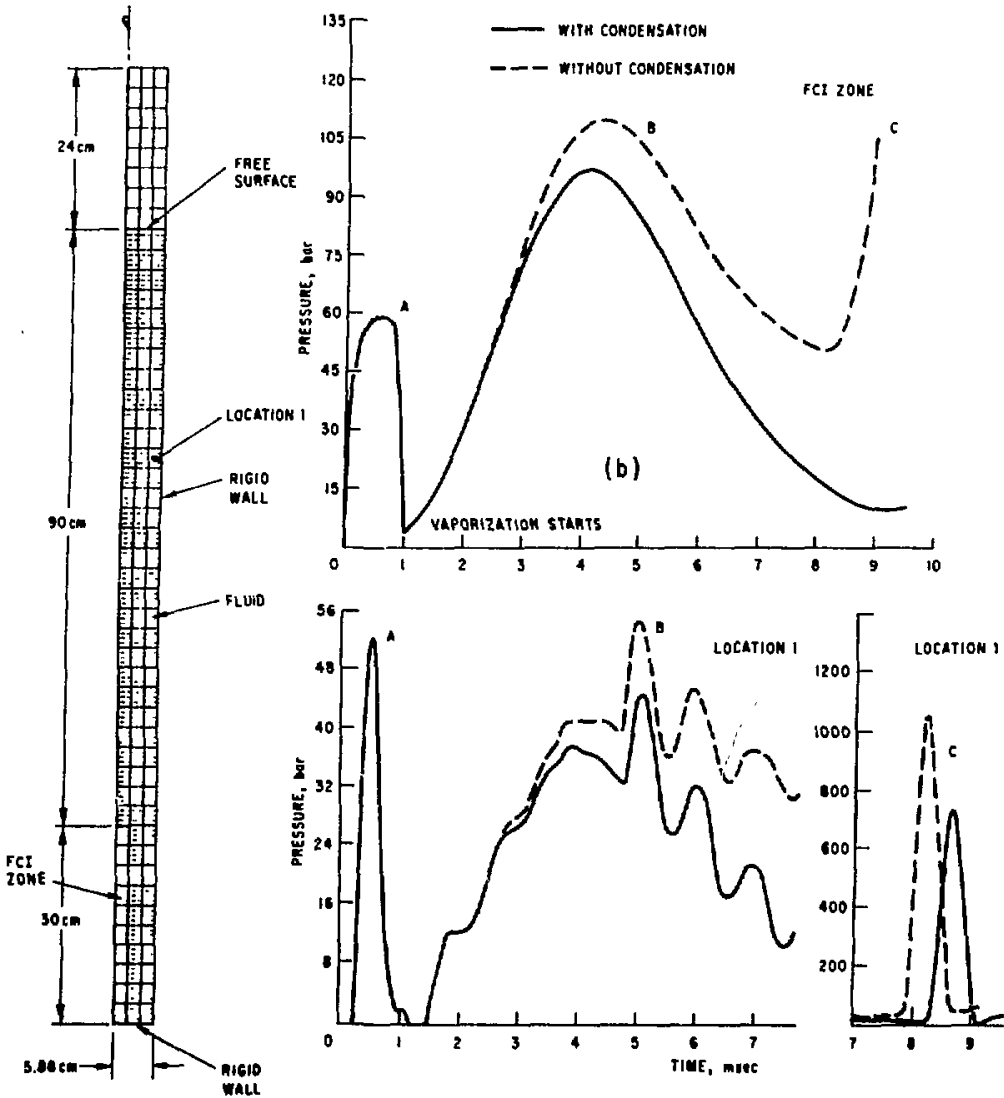

(0)

(c)

(d)

Fig. 34. Model of a Single Subassembly and Pressure Histories 
Fig. 35

Marker-Particle Configurations (with Condensation)

Fig. 36

Schematic of Shock Tube (not to Scale)

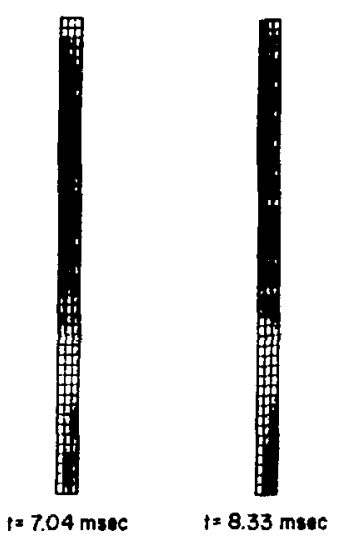

Rigid

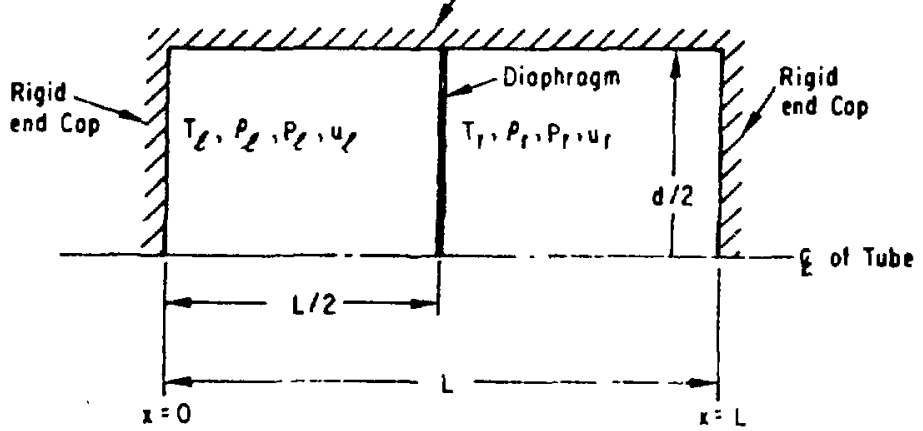

$P_{e}=100$ bors

$P_{r}=0.5 \cdot P_{f}=002905 \mathrm{gm} / \mathrm{cm}^{3}$

$P_{e}=00581 \mathrm{gm} / \mathrm{cm}^{3} \quad T_{p}=T_{p}=600 \mathrm{~K}$

$P_{f}=0.5 \cdot P_{l}=50$ bors

$u_{\ell}=u_{r}=4303 \times 10^{6} \mathrm{erg} / \mathrm{gm}$

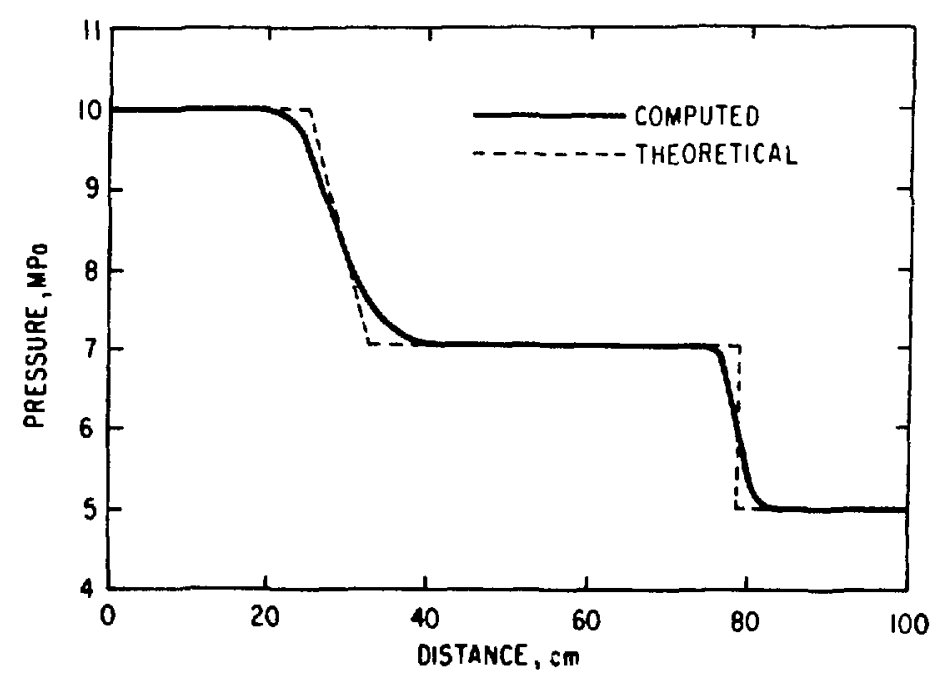

Fig. 37

Pressure Profile at $500 \mu \mathrm{s}$ 


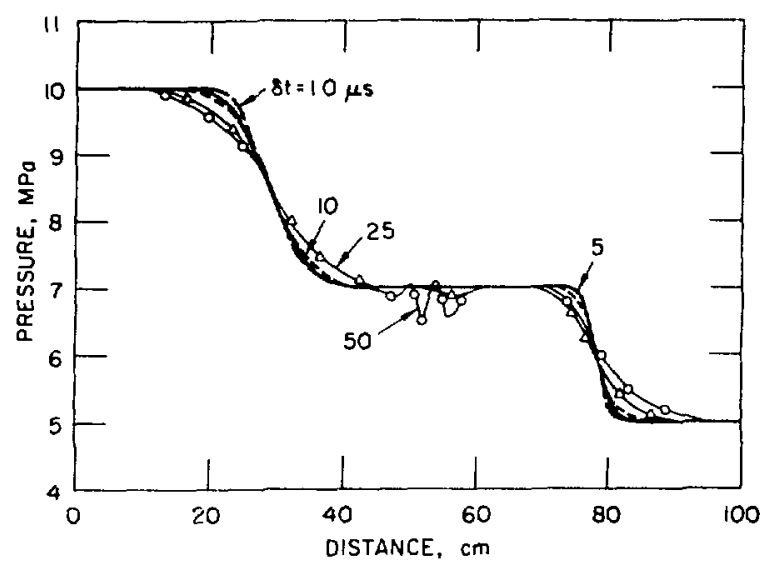

Fig. 38

Pressure Profiles at $500 \mu \mathrm{s}$ for Various Time Steps

Fig. 39

Simplified Test A pparatus for Bubble Motion

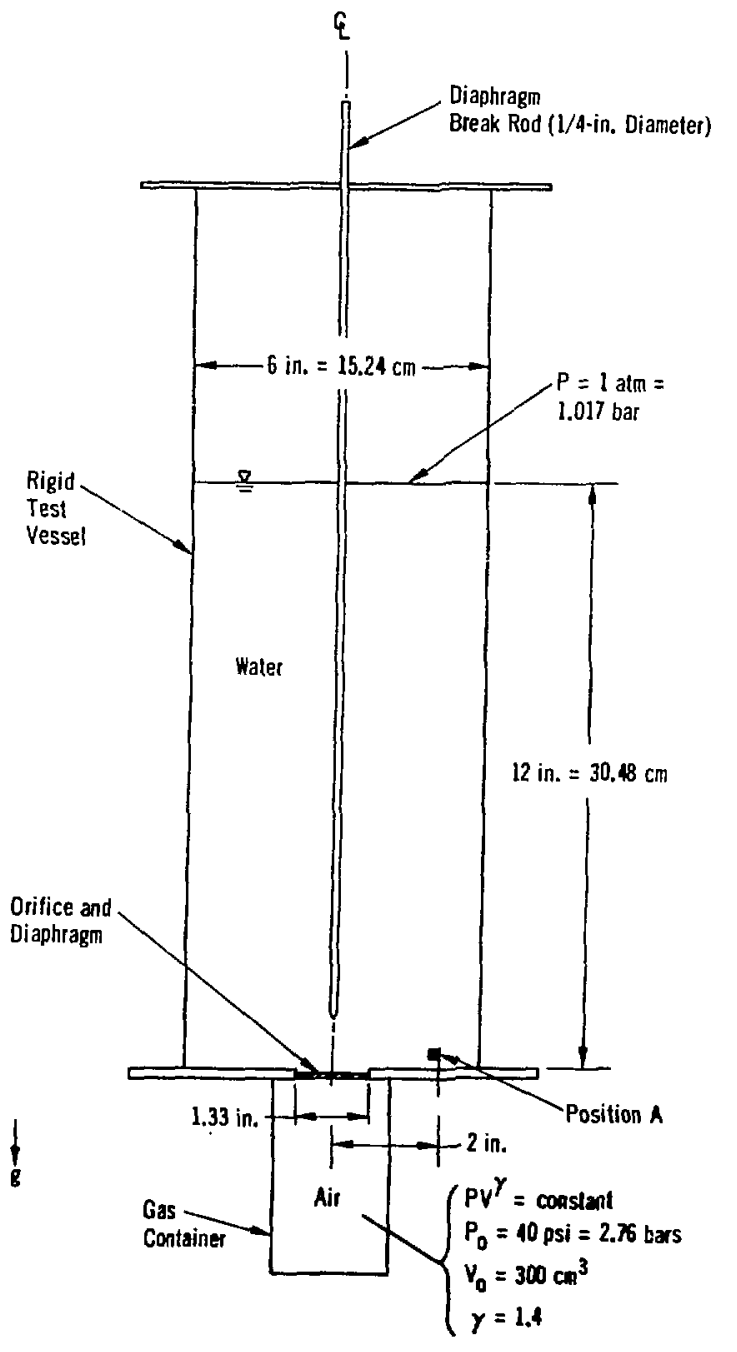




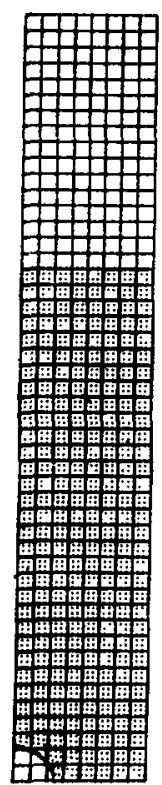

Time $=1.87$ msec

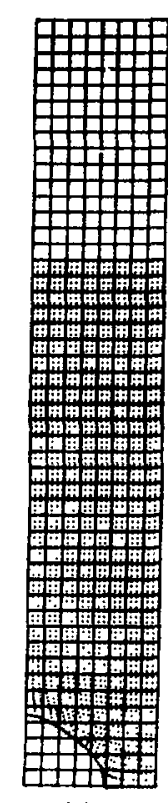

6.87

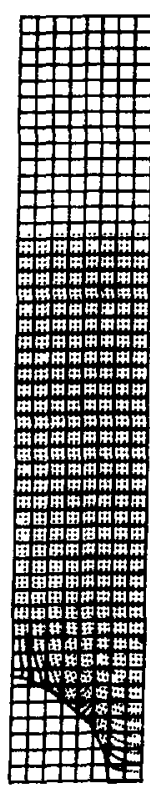

II.
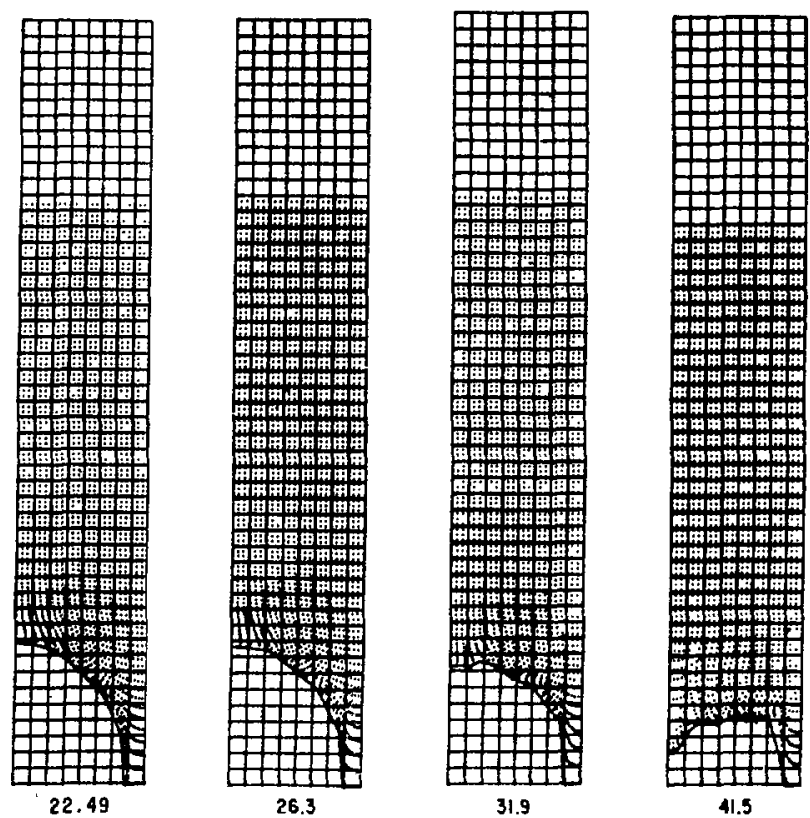

Fig. 40. Bubble and Coolant Configurations at Seven Different Times

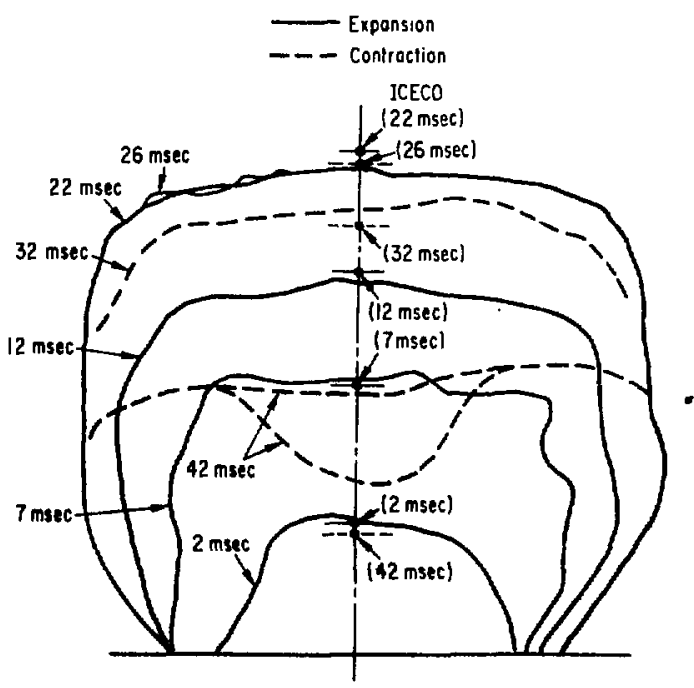

Fig. 41. Experimental Bubble Configurations at Seven Different Times

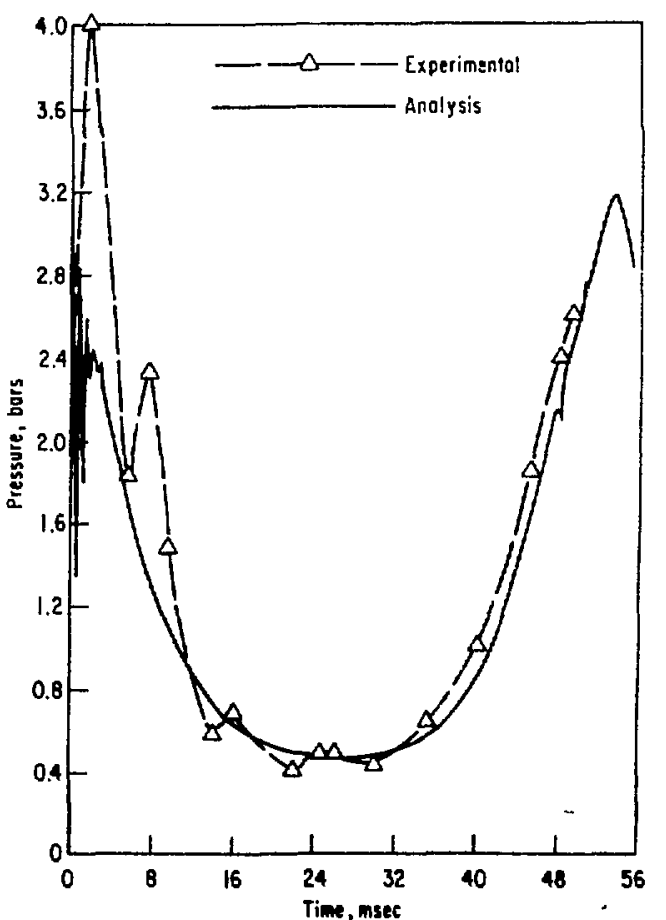

Fig. 42. Pressure Histories at Location $A$ 


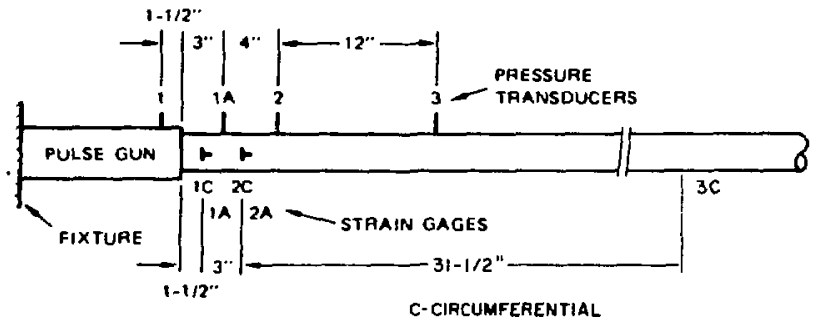

Fig. 43. Configuration of the Elastic-Plastic Pipe

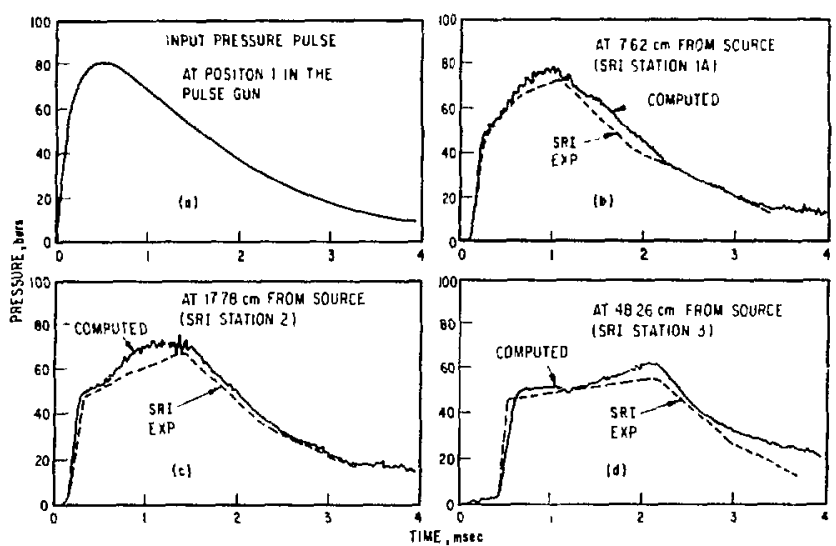

Fig. 44. Pressure Histories at Various Locations in the Elastic-Plastic Pipe
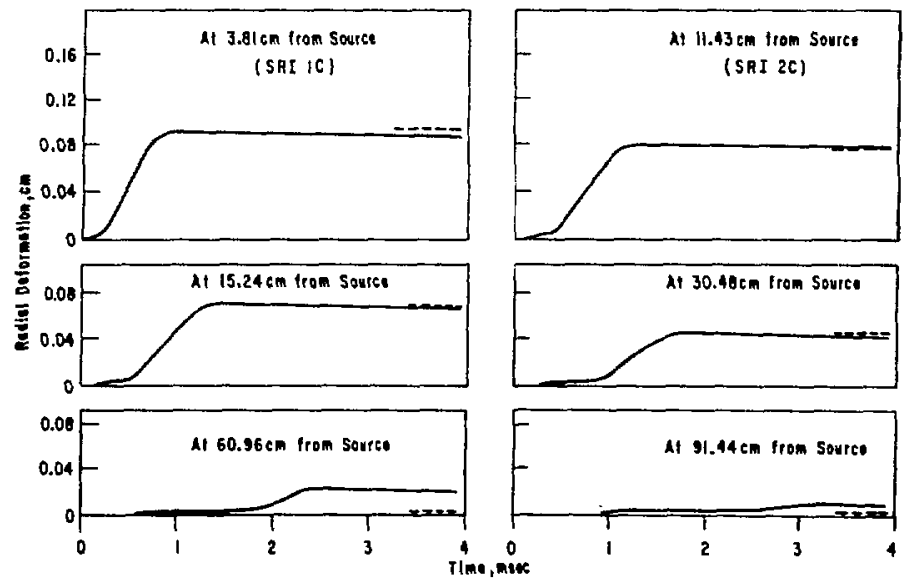

Fig. 45. Radial Deformations at Six Positions (-ICECO: -- SRI Test) 


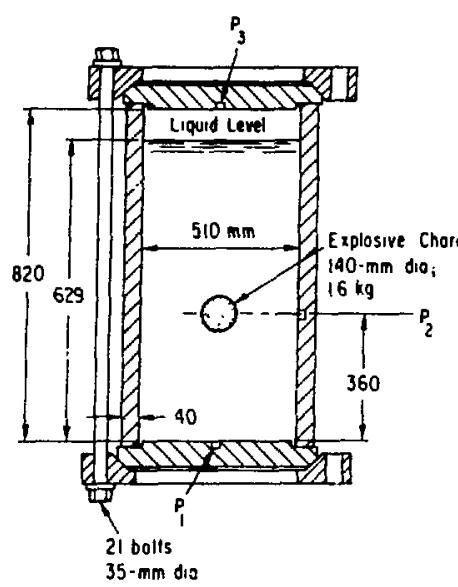

(a)

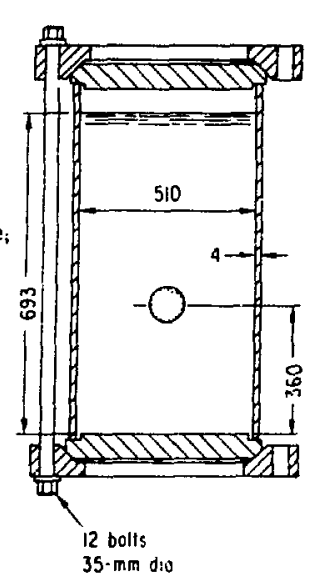

(b)

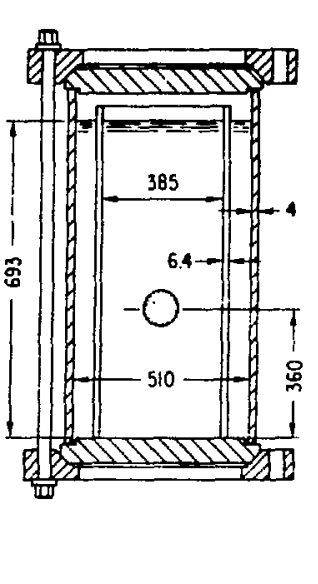

(c)

Fig. 46. Test configurations of the SNR-300 Reactor Models

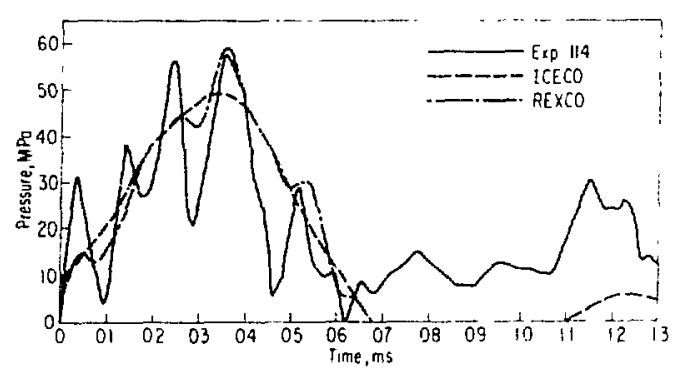

Hig. 47. Comparicon of Pressurc at $r=10 \mathrm{~cm}$ and $z=0$. (Delay of $0.2 \mathrm{~ms}$ ).

TIME $=0 \mathrm{~ms}$

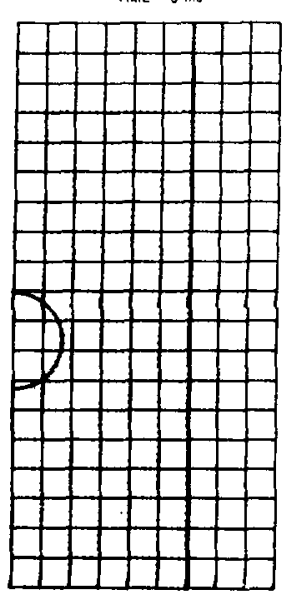

(1)

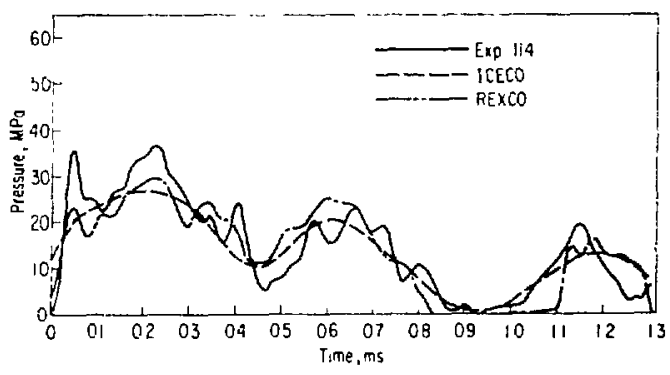

Fig. 48. Comparison of Pressure at $\mathrm{r}=25.5 \mathrm{~cm}$ and $z=36 \mathrm{~cm}$. (Delay of $0.12 \mathrm{~cm}$ ).

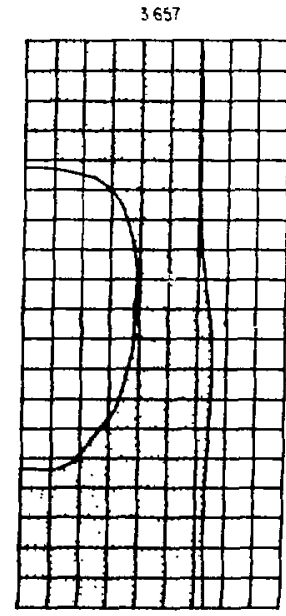

(c)

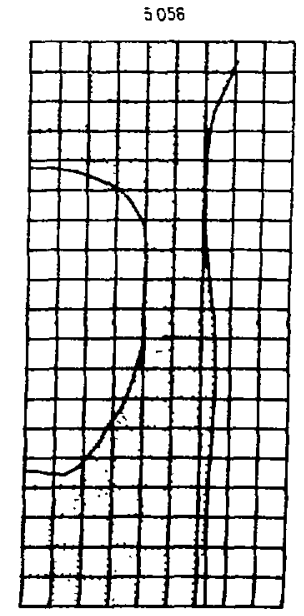

(d)

Fig. 49. Reactor Configurations at Three Different Times (No Spillage) 
(0)

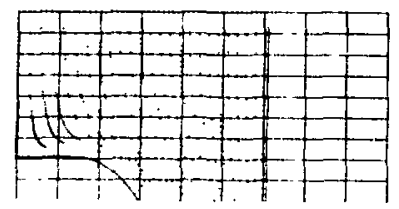

(b)
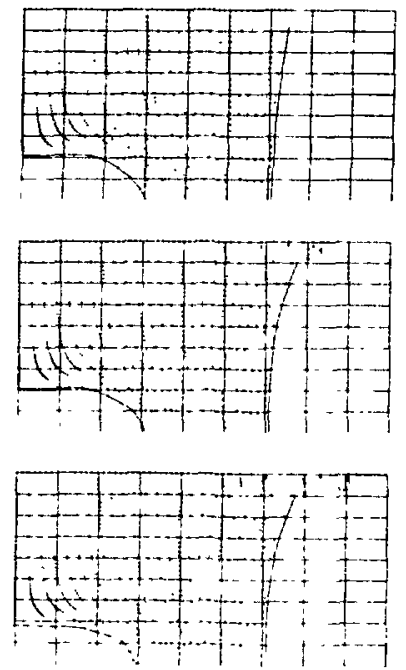

TIME $=3657 \mathrm{~ms}$

3857

Fig. 50

Reactor Configurations at Three Different Times (with Spillage)

4456

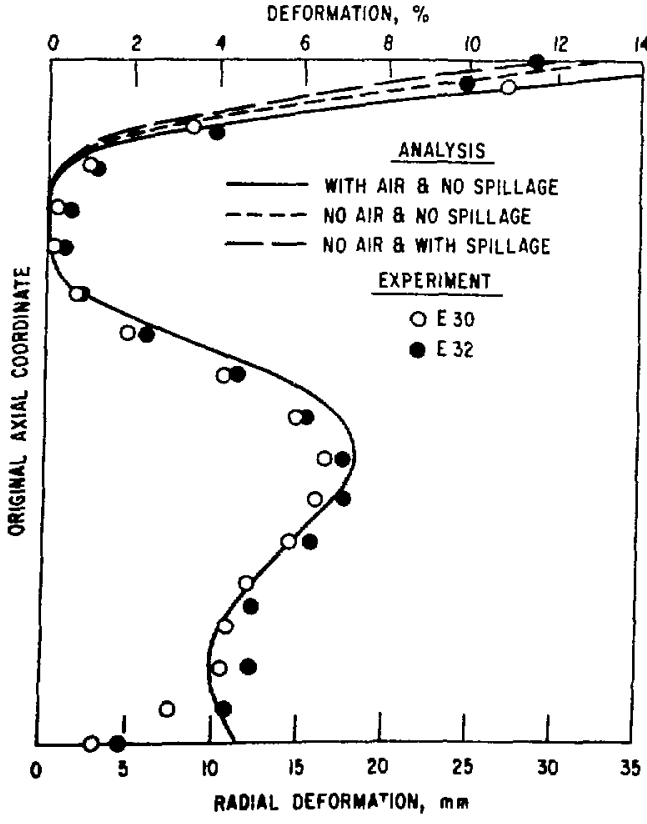

Fig. 51. Comparison of the Permanent Vesse Deformations (Thin-Vessel Model)

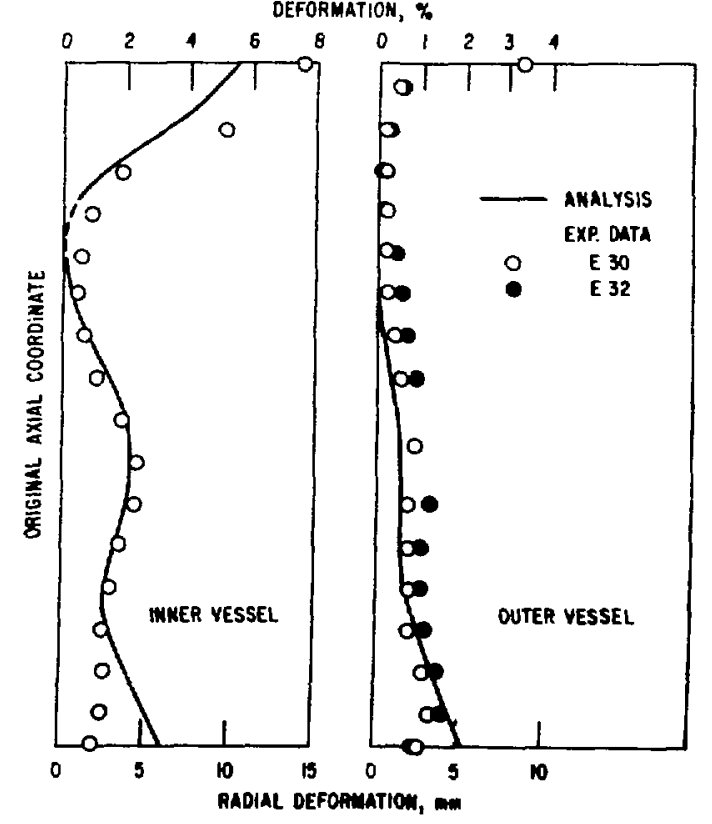

Fig. 52. Comparison of the Permanent Vessel Deformation (Double-Vessel Model) 


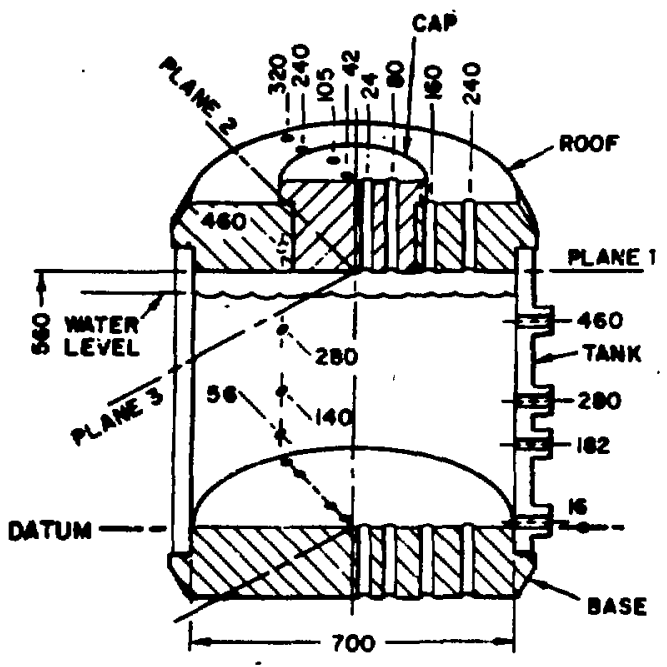

(a)

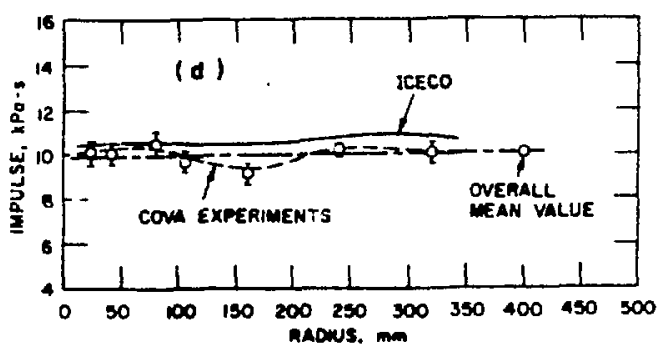

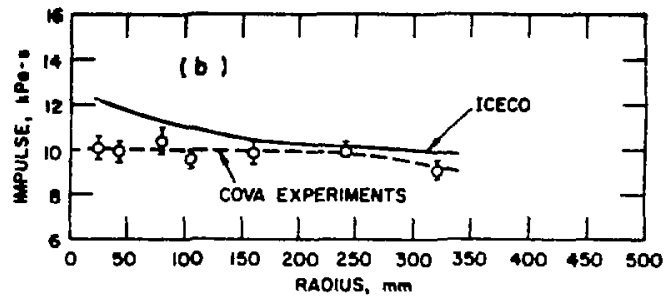
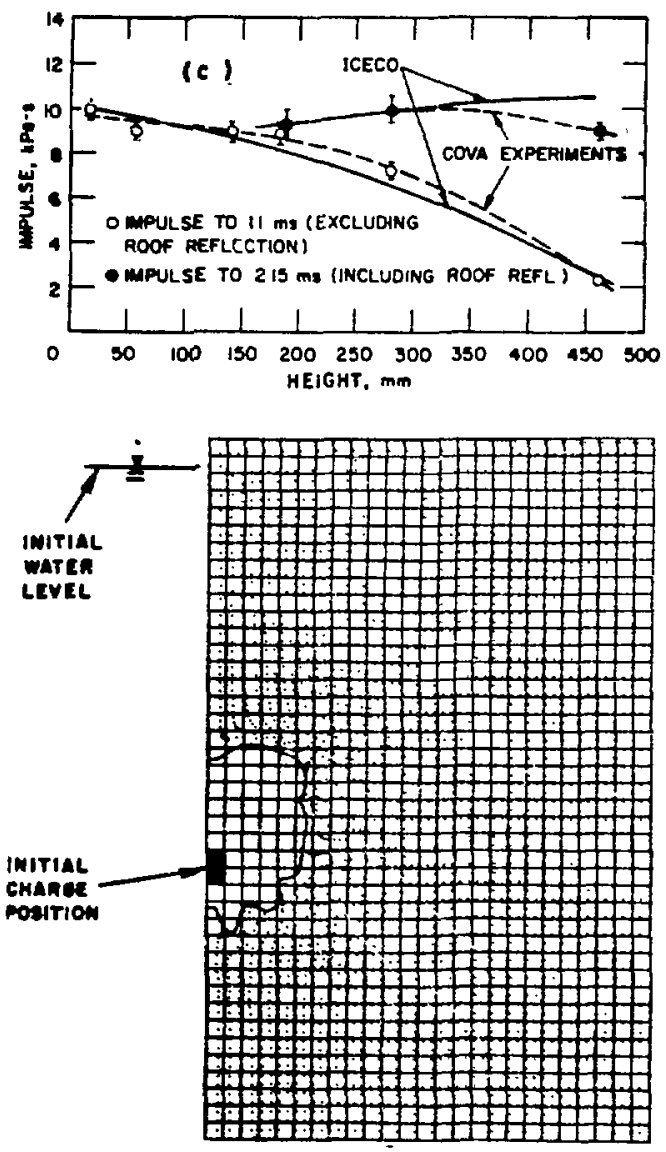

$+1.0 \mathrm{ma}$

(a)

Fig. 53. (a) Test Model of the COVA Overstrong Vessel Experiment: (b-d) Inpulses on the Floor, Wall, and the Roof, (e) Configuration at $1 \mathrm{~ms}$ 


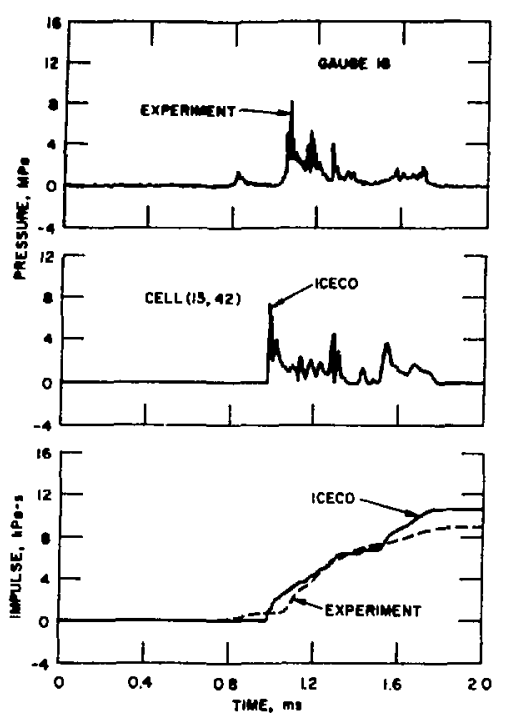

Fig. 54. Comparison of Pressure Histories at Roor Gauge \#18 $(\mathrm{r}=160 \mathrm{~mm}$ )

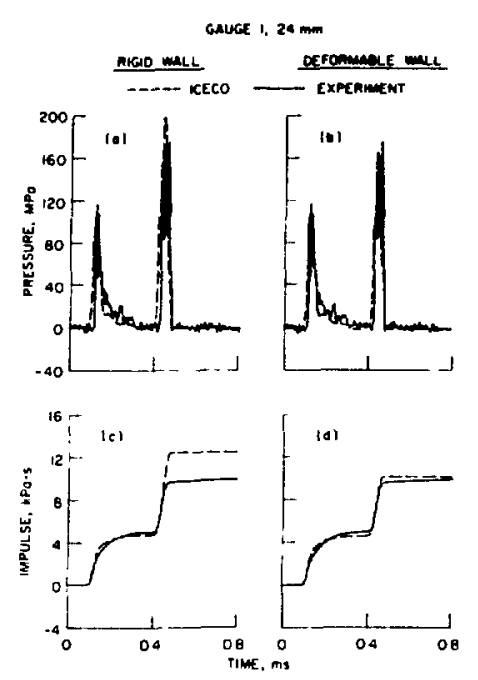

Fig. 55. Pressure and lmpulse Historics for l:loor Gauge \#1 $(\mathbf{r}=24 \mathrm{~mm})$

SM 2 WITH INSTRUUENTATION

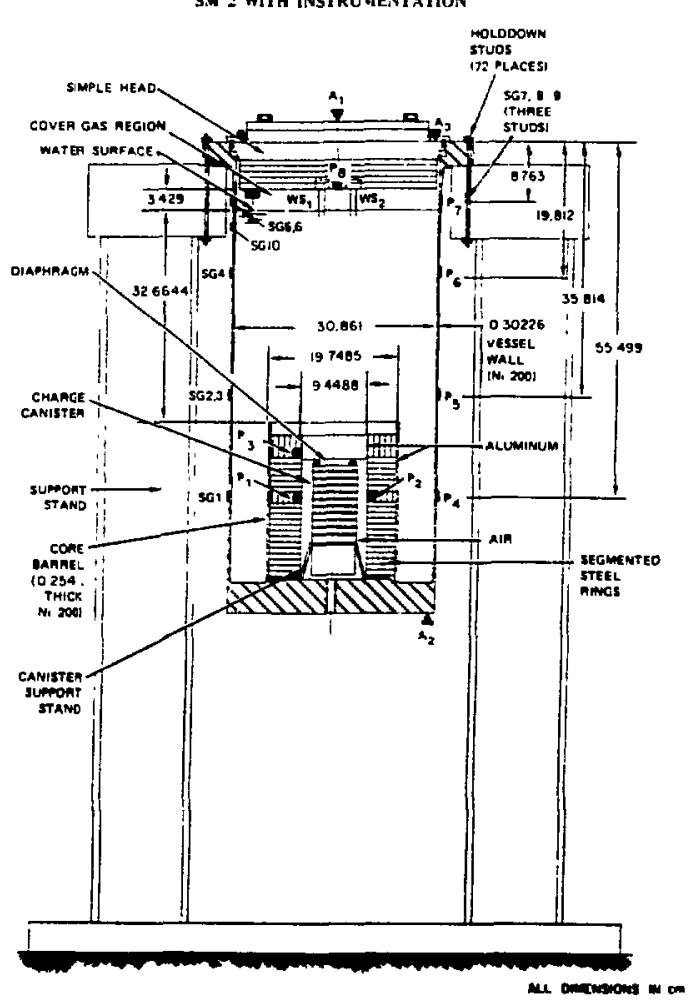

Fig. 56. Impulses at Floor Gauges 


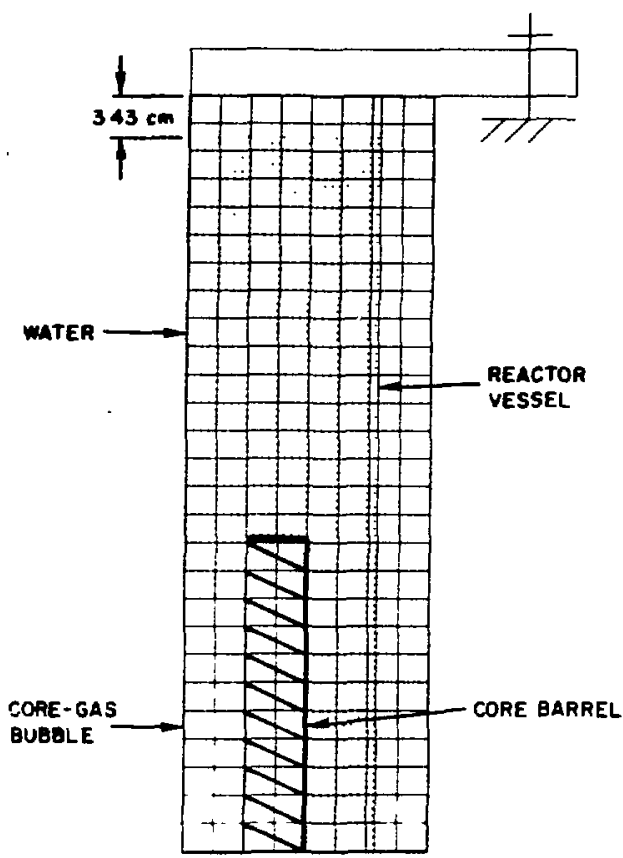

$t=0 \mathrm{~ms}$

(0)

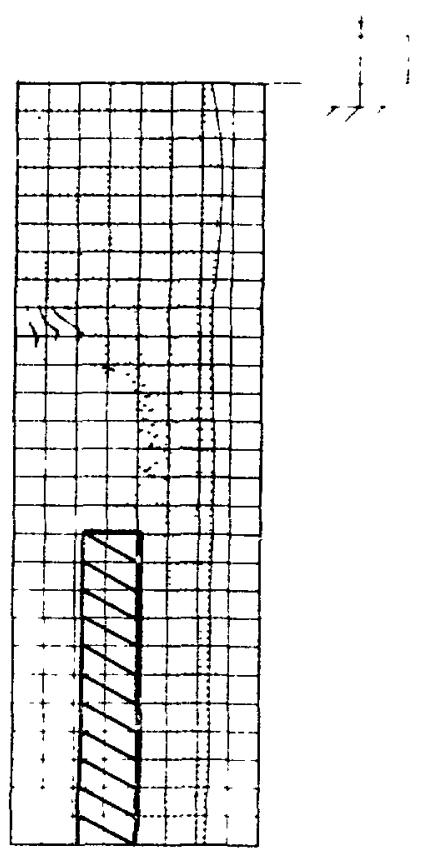

$1=3.5 \mathrm{~ms}$

(b)

Fig. 58. Configurations at Two Different Times

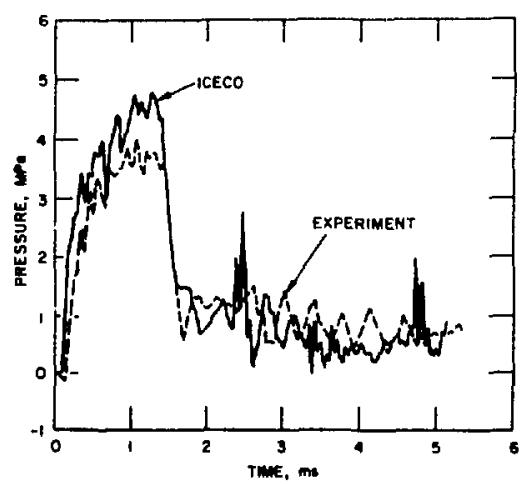

Fig. 59. Pressure Histories at Gauge P-5

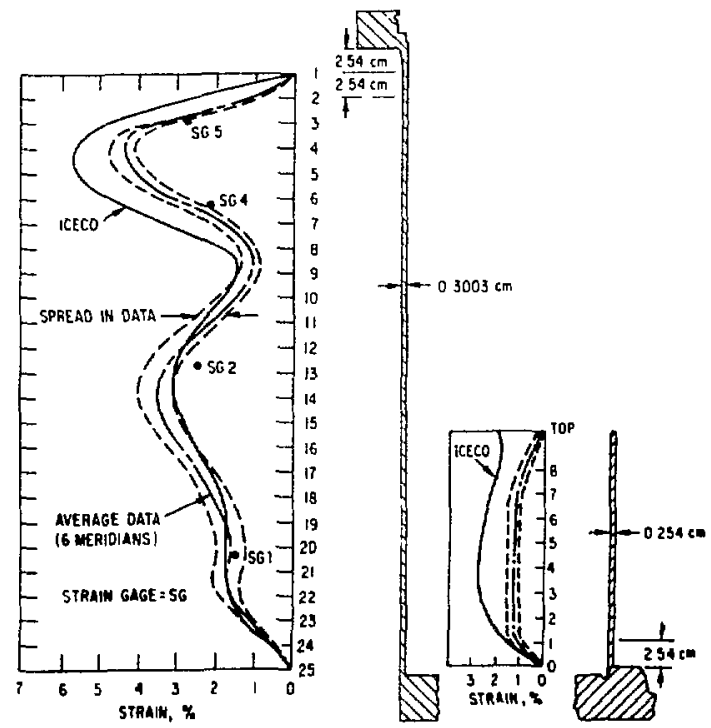

Fig. 60. Deformed Shape Profile of SM-2 

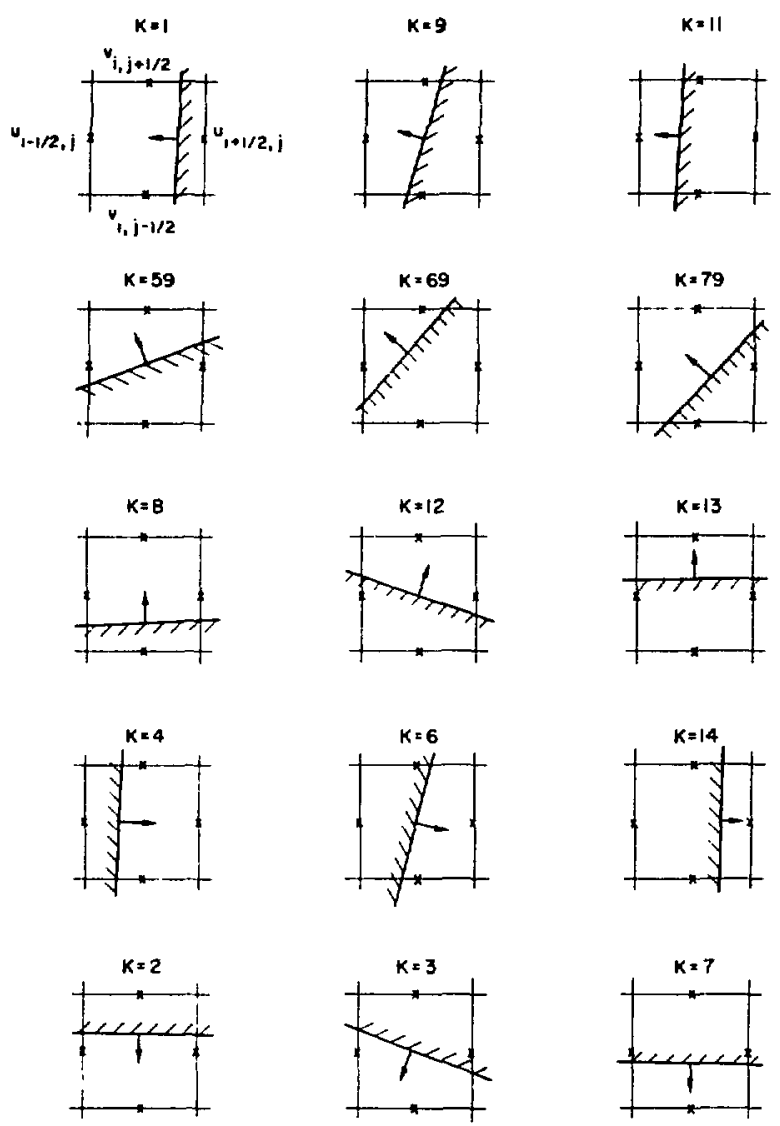

Fig. 61. Different Type of Elags at the Deformable Boundaries
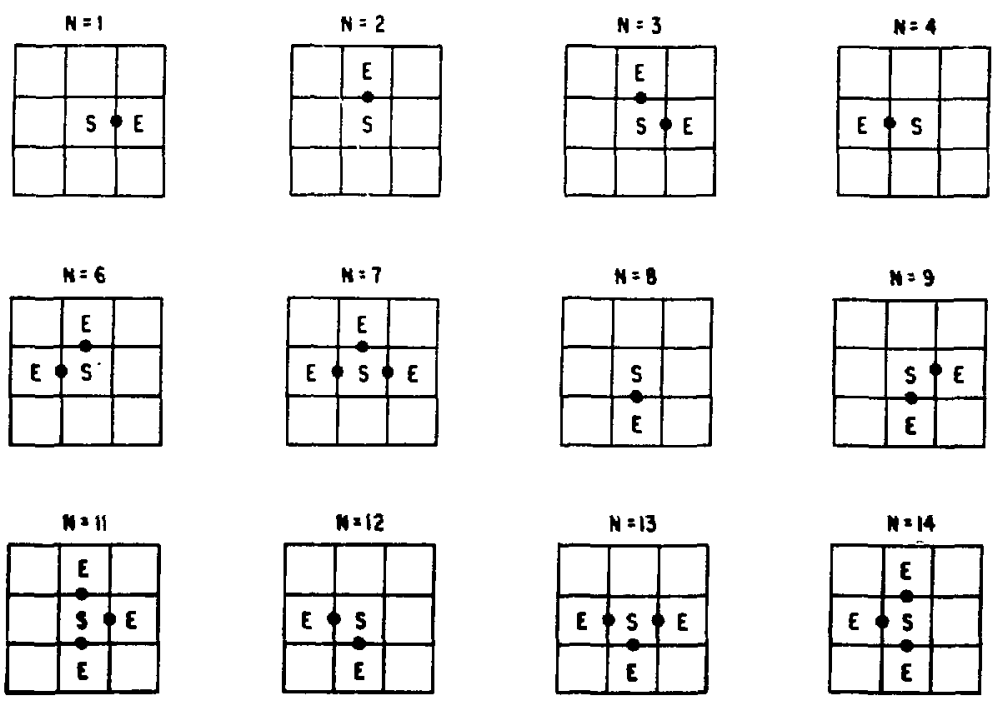

Fig. 62. Conventional Cell Flags Used Near the Free Surface 

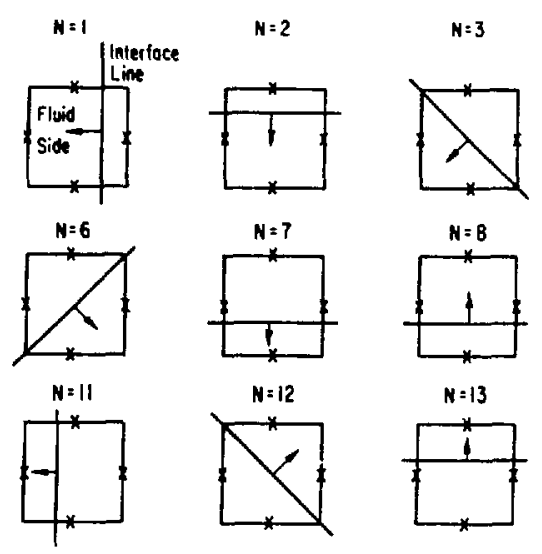

Fig. 63. Detailed Cell Flags Used Near the Free Surface

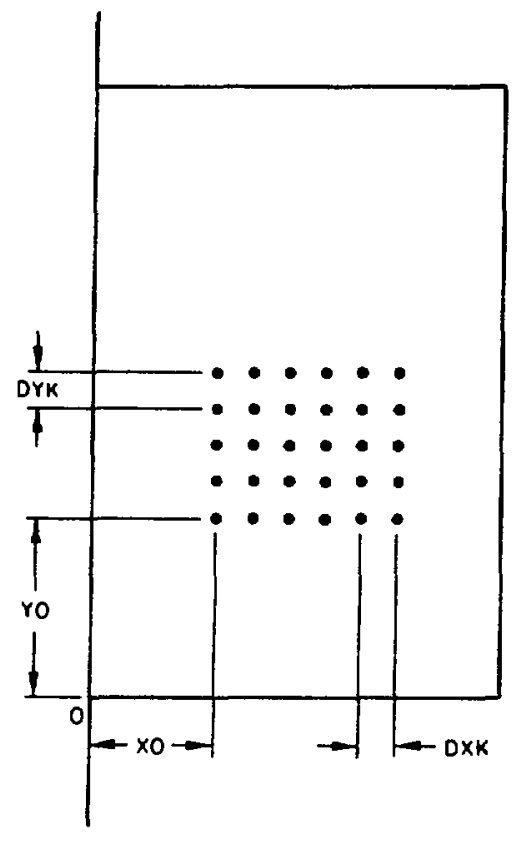

Fig. 64. Marker Particle Setup

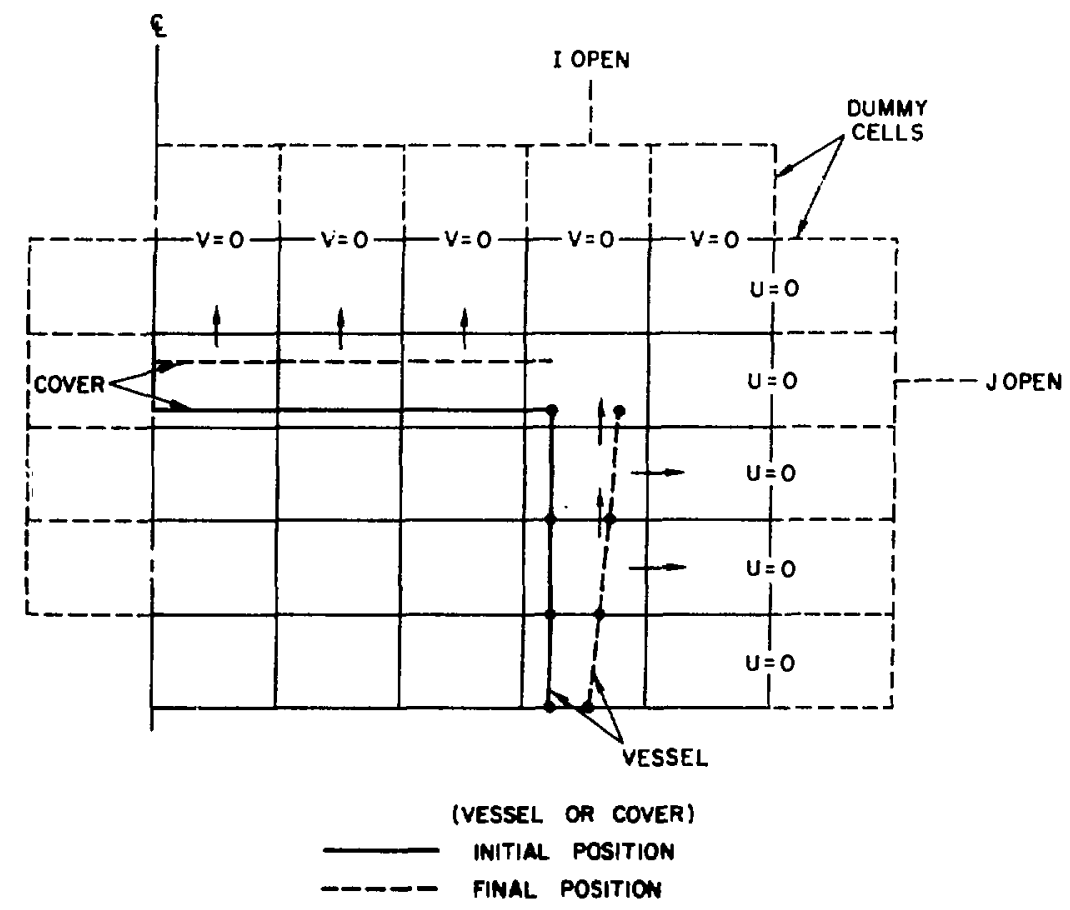

Fig. 65. Computing Mesh for Problems with Flexible External Boundaries 

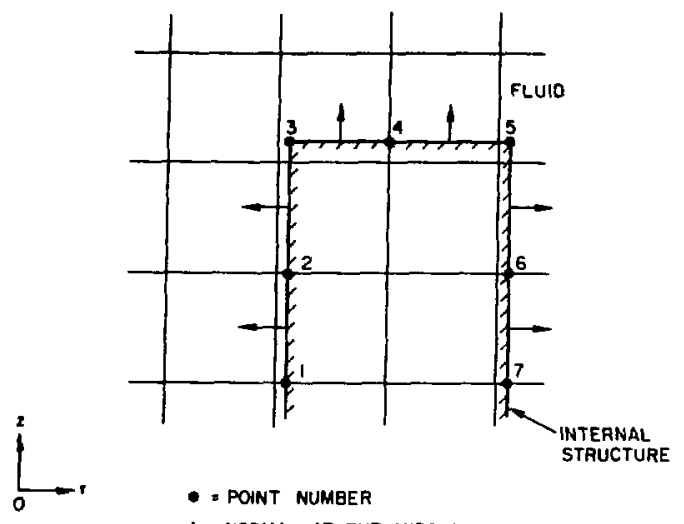

- - point number

= MORMAL at THE MIDOLe

Fig. 66. Computing Mesh for Problems with Deformable Internals

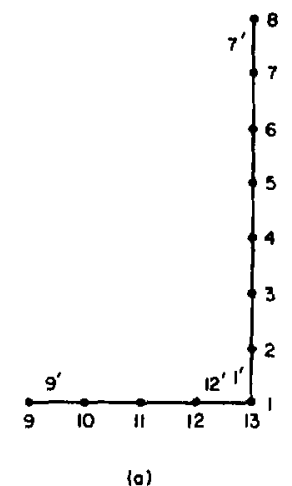

Fig. 67. Numbering System for Treating Fluid-Structure Interaction with Multiple Load Lines

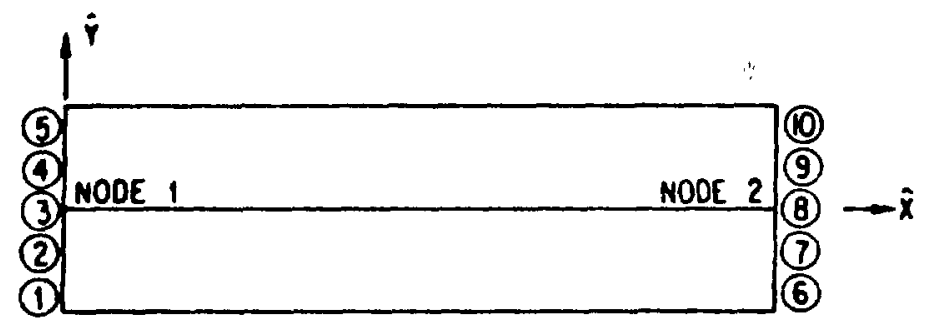

\begin{tabular}{|l|c|c|c|c|c|c|c|c|c|c|}
\hline & $(1)$ & $(2)$ & $(3)$ & $(4)$ & $(5)$ & $(6$ & $(7)$ & $(8)$ & $(9)$ & $(10$ \\
\hline$\hat{\epsilon}_{\mathrm{x}}$ & 1 & 2 & 3 & 4 & 5 & 6 & 7 & 8 & 9 & 10 \\
\hline$\hat{\sigma}_{\mathrm{x}}$ & 11 & 12 & 13 & 14 & 15 & 16 & 17 & 18 & 19 & 20 \\
\hline$\hat{\epsilon}_{\ell}$ & 21 & 22 & 23 & 24 & 25 & 26 & 27 & 28 & 29 & 30 \\
\hline$\hat{\sigma}_{8}$ & 31 & 32 & 33 & 34 & 35 & 36 & 37 & 38 & 39 & 40 \\
\hline YIELO ICI & 41 & 42 & 43 & 44 & 45 & 46 & 47 & 48 & 49 & 50 \\
\hline
\end{tabular}

Fig. 68. Component Number for the Time-History Plot 